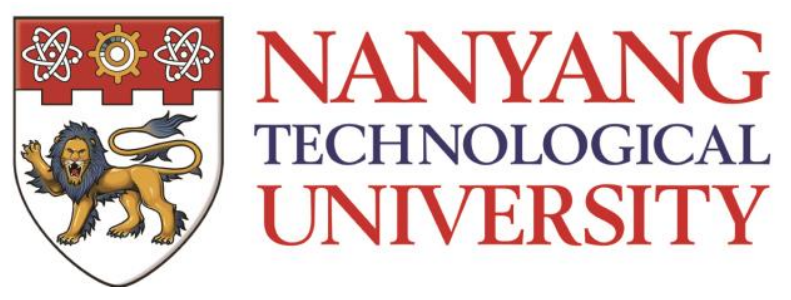

\title{
OPERATION AND CONTROL OF MULTI-AREA MULTI-MICROGRID SYSTEMS
}

\section{THOMAS JOHN}

School of Electrical and Electronic Engineering

A thesis submitted to the Nanyang Technological University in partial fulfillment of the requirement for the degree of Doctor of Philosophy 



\section{ACKNOWLEDGEMENTS}

This thesis would not have been possible without the help and guidance of several individuals who in one way or another contributed and extended their valuable assistance in the preparation and completion of this study.

First, I wish to express my sincere gratitude to my supervisor, Associate Professor So Ping Lam, for his continuous insight, enthusiasm and encouragement throughout the whole research program. His deep knowledge on electric power systems, power quality and smart grids has been very valuable for my research. He has always been my inspiration and role model as I faced different obstacles in the completion of this research program.

Then, I would like to thank my fellow PhD students, Wang $\mathrm{Yu}$, Wang Benfei and Ravi Kishore for their valuable suggestions and supports throughout my research work. I would also like to extend my gratitude to my group members, namely, Dr. Nandha Kumar, Dr. Tan Kuan Tak, Dr. Sivaneasan Balakrishnan and Mr. Peng Xiaoyang for the fruitful discussions on my research work.

Furthermore, I am thankful for Nanyang Technological University to grant me the research scholarship so that I can have this opportunity to pursue my $\mathrm{PhD}$ in this excellent university. I am also thankful for the technical support provided by the laboratory staff Chia-Nge Tak Heng and Foo Mong Keow, Thomas in Clean Energy Research Laboratory. They generously offered their help during my study in the laboratory.

Finally, I would like to extend my sincere appreciation and gratitude to my family, friends and colleagues who have always been supportive of my $\mathrm{PhD}$ studies and research. 


\section{ABSTRACT}

There has been a widespread deployment of microgrids around the world in recent years. Microgrids form a local area power distribution system with distributed generations, energy storage systems and controllable loads. The next stage of innovation in the field of microgrid systems is the interconnection of several AC and DC microgrid systems spread over large geographical distances to form multi-area multi-microgrid (MMG) systems, which will satisfy the ever increasing global energy demands. There are several benefits associated with these MMG systems such as improved reliability and security of power supply, mutual power sharing and reduced investment in new generating capacity. For the effective operation and control of these MMG systems during different modes of operation, effective methods of power, voltage and frequency controls are essential.

First, different system architectures are proposed for AC/AC multi-area MMG system, consisting of interconnected AC microgrids and for AC/DC multi-area MMG system, consisting of both interconnected AC and DC microgrids. Control systems consisting of centralized and local controllers are proposed for the effective control of load bus voltages and frequency, inverter and converter power outputs, and power exchange between the interconnected microgrids in each of these MMG systems. The local control of the converters and inverters in these MMG systems is realized using a state-space model based control algorithm, namely model predictive control (MPC). The proposed MPC algorithm, unlike the existing MPC algorithms, is independent of grid, line and load impedances in the MMG systems. In comparison with conventional proportional-integral (PI) control methods, the proposed MPC algorithm gives smaller tracking error, shorter settling time, and better steadystate and transient responses in different operating modes of the interconnected microgrids such as grid-connected and islanded modes. In addition to the system architectures and control systems, different load shedding schemes are also proposed for the MMG systems. An underfrequency load shedding scheme is proposed for the AC/AC multi-area MMG system for effective voltage and frequency regulation during AC microgrid islanding. Also, an undervoltage load shedding scheme is proposed for the AC/DC multi-area MMG system for effective voltage and power regulation during DC microgrid islanding.

Then, various simulation studies are conducted to test the operation and control of these MMG systems under different operating conditions such as microgrid islanding, power 
exchanges, load changes, load shedding and line outages. The simulation studies show that the developed control systems in these MMG systems can achieve good control performance and effective voltage, frequency and power regulation under different operating conditions. Thus, the effectively controlled multi-area MMG systems are capable of fulfilling basic objectives such as improved reliability and security of power supply, enhanced voltage and frequency stability, and effective dynamic islanding.

Finally, to solve the various power quality issues such as current distortion, voltage distortion, voltage sag, voltage unbalance and low power factor in the proposed AC/AC multi-area MMG system, a new power quality improvement method is proposed. A new method of non-local harmonic current and reactive power compensation using a series-shunt network device (SSND) is proposed for the AC/AC multi-area MMG system. Even though local harmonic current and reactive power compensation methods are available, non-local compensation methods, based on their several advantages, are alternatives to be necessarily considered in the future for large MMG systems, which consist of widely dispersed loads. SSND consisting of series and shunt inverters is installed in the line interconnecting two microgrids in the AC/AC multi-area MMG system. A state-space model based MPC algorithm is used for the proposed power quality improvement scheme to regulate various parameters such as output voltage, frequency, current and power of multiple inverters and converters in the MMG system integrated with the SSND. The power flow and power quality control functions of the SSND are analyzed theoretically to understand the different capabilities of SSND in harmonic current and reactive power compensation and in voltage disturbance isolation in the MMG system.

Several simulation studies are conducted to demonstrate the effective operation of SSND using MPC in the proposed AC/AC multi-area MMG system. From these simulation studies, it is verified that SSND can effectively achieve local and non-local harmonic current and reactive power compensation, and can also isolate one microgrid from voltage disturbances such as voltage distortion, voltage sag and voltage unbalance occurring in the adjacent microgrid. In addition, SSND can provide emergency real power support during islanding of a microgrid in the MMG system. 


\section{TABLE OF CONTENTS}

ACKNOWLEDGEMENTS ...

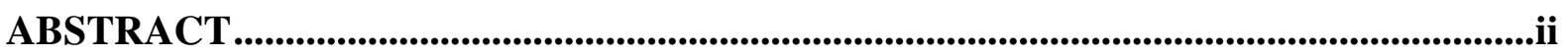

TABLE OF CONTENTS ...........................................................................................................iv

LIST OF FIGURES ............................................................................................................ viii

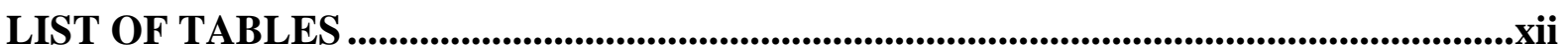

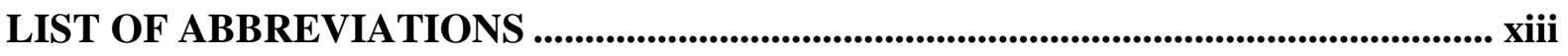

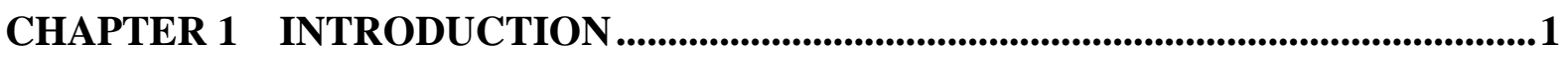

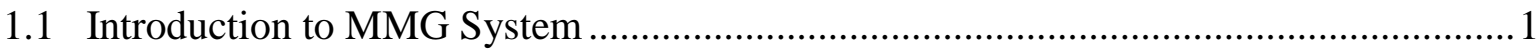

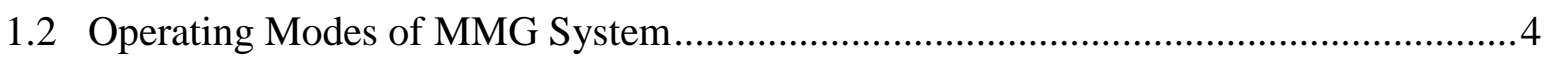

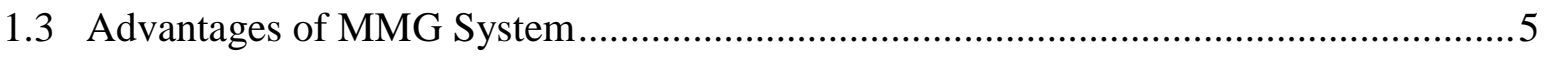

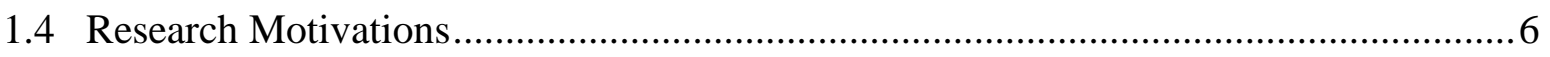

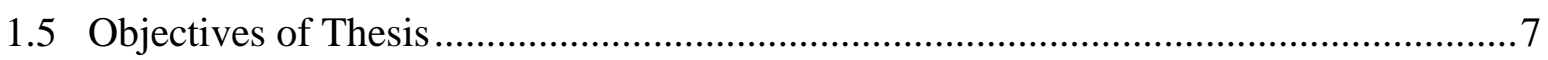

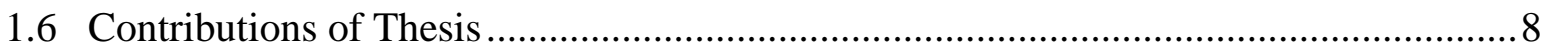

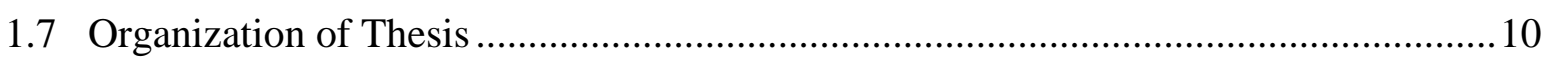

CHAPTER 2 LITERATURE REVIEW .................................................................................12

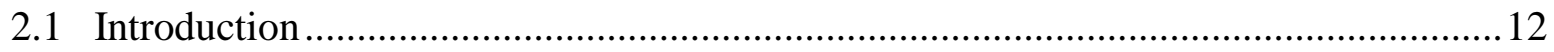

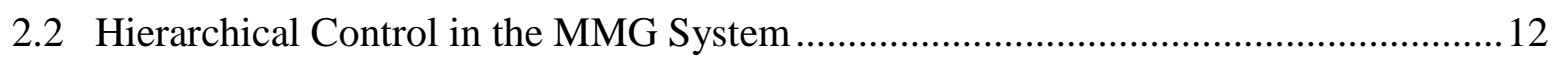

2.2.1 Data Exchange among Different Controllers ................................................ 14

2.3 Centralized and Decentralized Control Schemes in the MMG System ....................... 15

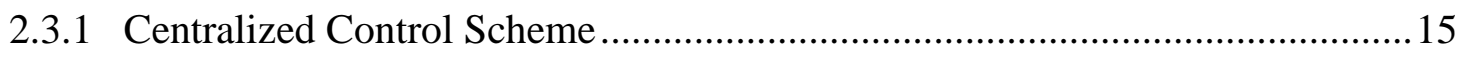

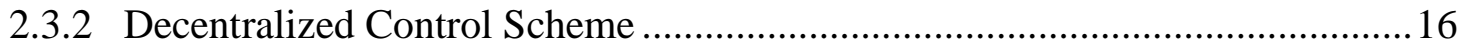

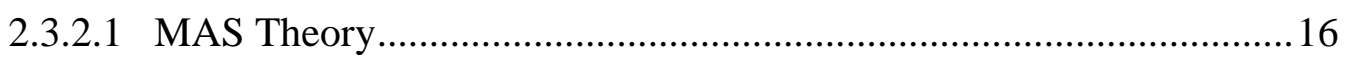

2.4 Advantages and Disadvantages of Centralized and Decentralized Control Schemes .. 17

2.4.1 Advantages and Disadvantages of Centralized Control Scheme...................... 17

2.4.2 Advantages and Disadvantages of Decentralized Control Scheme ................... 18

2.5 Control Schemes Adopted by Local Controllers in the MMG System.........................19

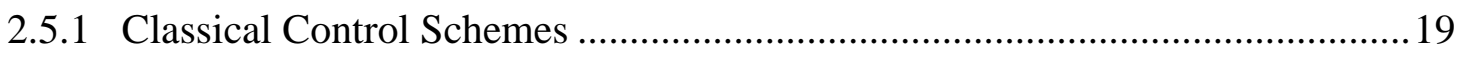


2.5.1.1 Hysteresis Current Control .............................................................. 19

2.5.1.2 Linear Control with Pulse Width Modulation (PWM) ......................20

2.5.2 Non-classical Control Schemes ................................................................22

2.5.2.1 Predictive Control Methods for Power Converters ...........................22

2.5.2.2 Model Predictive Control (MPC) ..................................................2 23

2.6 Operation Modes of Microgrids in the MMG System .............................................29

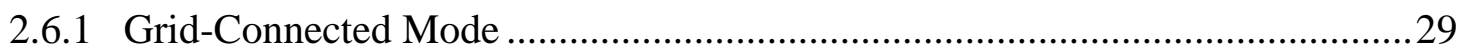

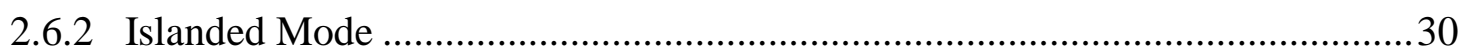

2.7 Control Modes of Microgrids in the MMG System ......................................................... 31

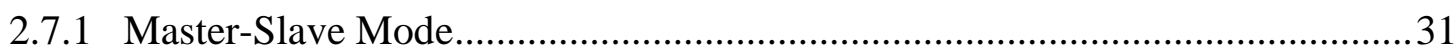

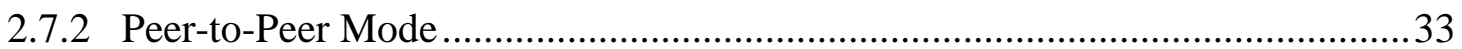

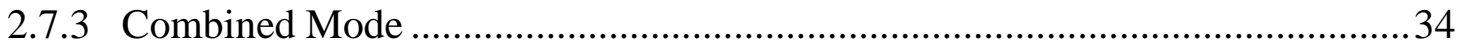

2.8 Different Types of MMG Systems - Conventional and Proposed MMG Systems ....... 35

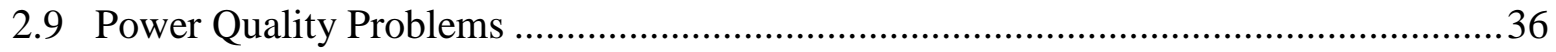

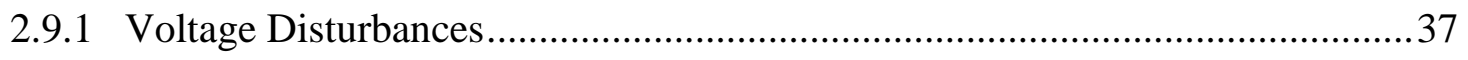

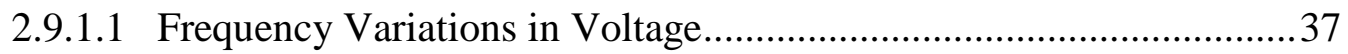

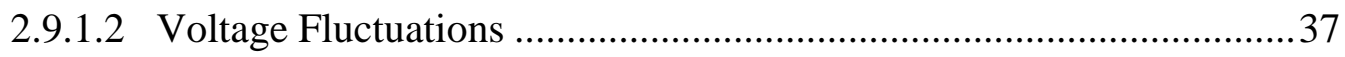

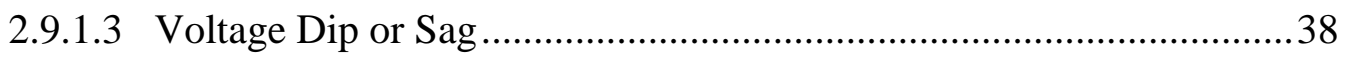

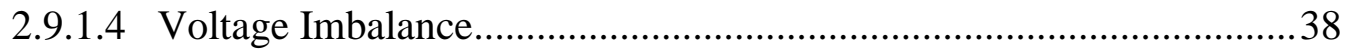

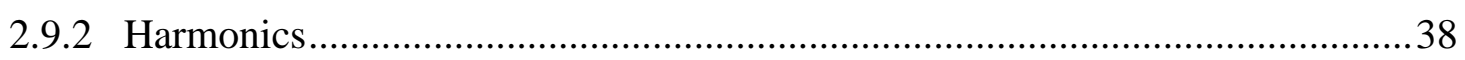

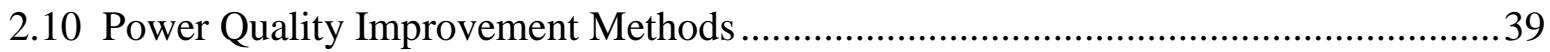

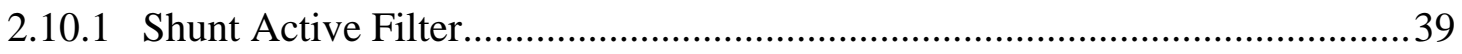

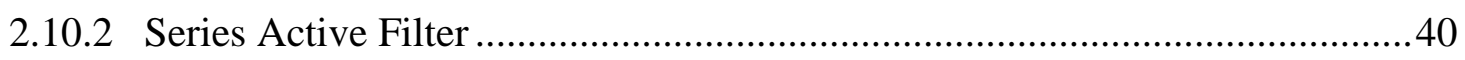

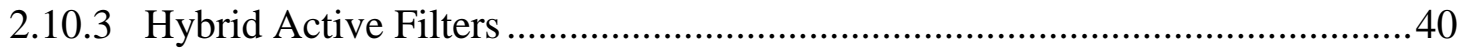

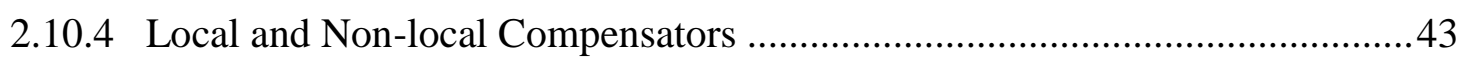

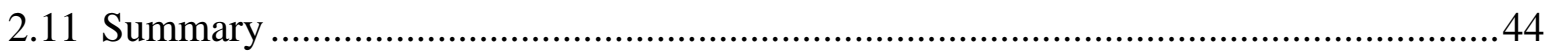

CHAPTER 3 OPERATION AND CONTROL OF AC/AC MULTI-AREA MMG

SYSTEM ..................................................................................................................................................46

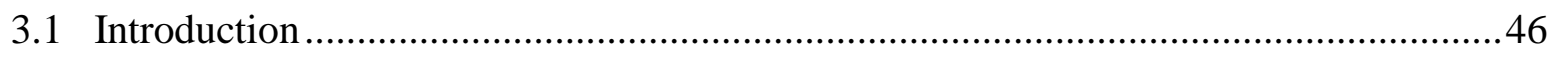




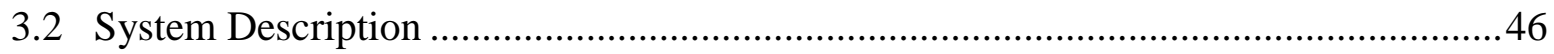

3.3 Modelling and Control of Inverters in the MMG System ........................................ 49

3.3.1 Flowcharts of the Proposed MPC Algorithm ............................................53

3.3.2 Procedures for Evaluation of Cost Function in the Proposed MPC Algorithm.56

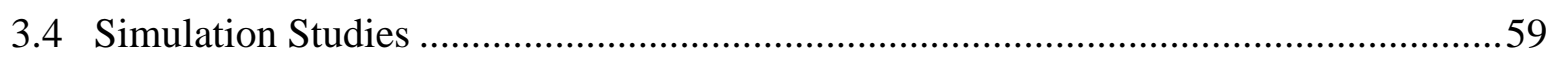

3.4.1 Test Case 1 - Islanded Microgrid Receiving Sufficient Power from Adjacent Grid-connected Microgrids

3.4.2 Test Case 2 - Islanded Microgrid Receiving Insufficient Power from Adjacent

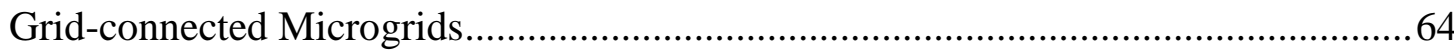

3.4.3 Test Case 3 - Failure of DG Inverter in Islanded Microgrid ............................67

3.5 Comparison of MPC and PI Controllers for an Inverter in the MMG System .............69

3.5.1 Comparison of MPC and PI Controllers for Test Case 2 ...............................72

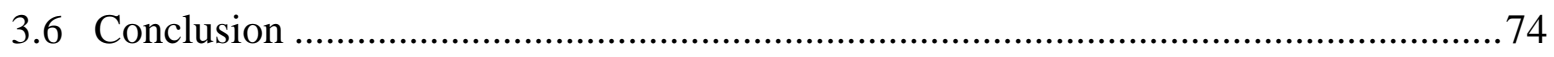

CHAPTER 4 VOLTAGE AND POWER CONTROL IN AN AC/DC MULTI-AREA

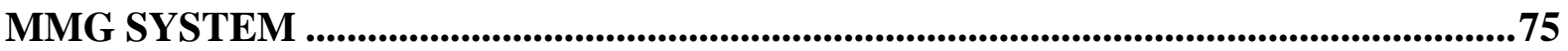

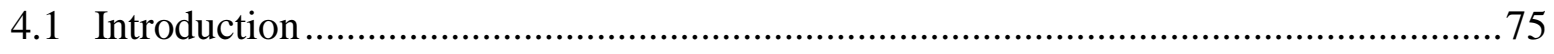

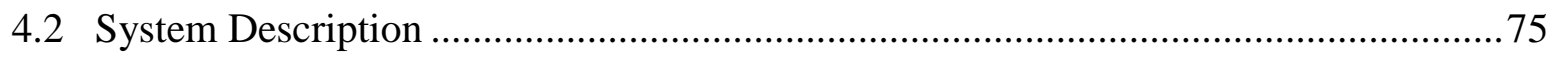

4.3 Modelling of Converters in the MMG System …................................................... 78

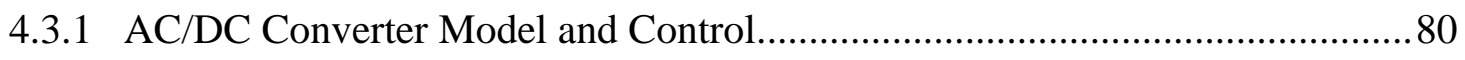

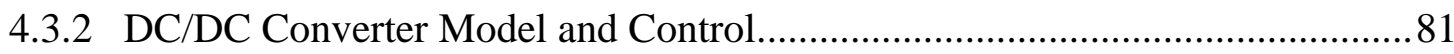

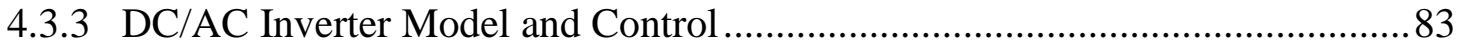

4.3.4 Objective Function and Constraints of DC/DC Converter and DC/AC Inverter

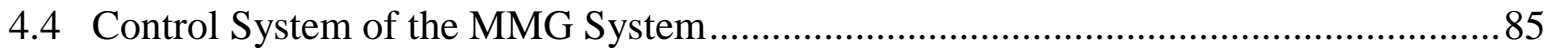

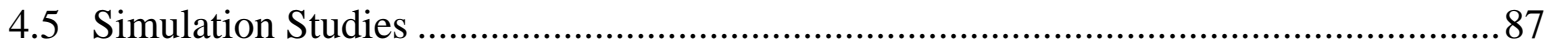

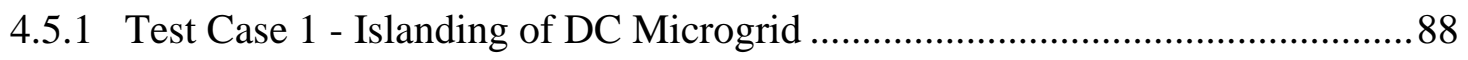

4.5.2 Test Case 2 - Power Exchange between DC and AC Microgrids ......................91

4.5.3 Test Case 3 - Undervoltage Load Shedding in Islanded DC Microgrid............95

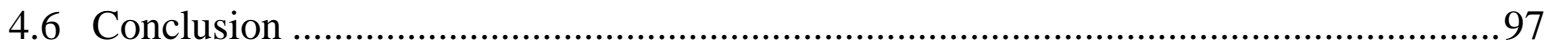


CHAPTER 5 SERIES-SHUNT NETWORK DEVICE FOR AC/AC MULTI-AREA

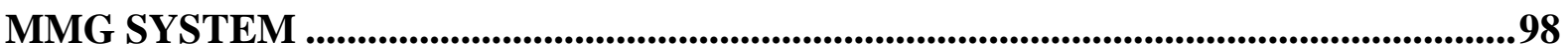

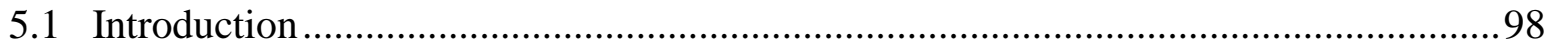

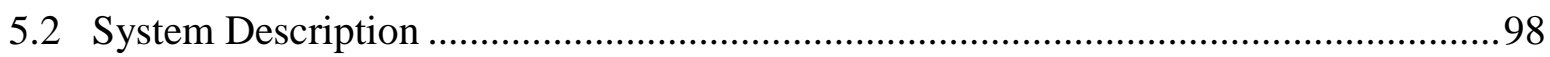

5.3 Modelling of Inverters in the Proposed System ................................................. 101

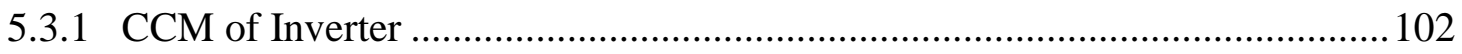

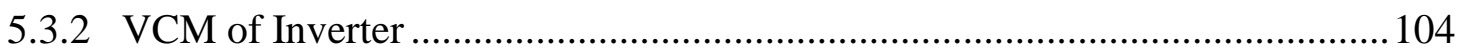

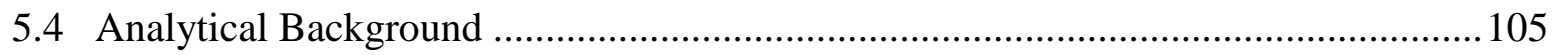

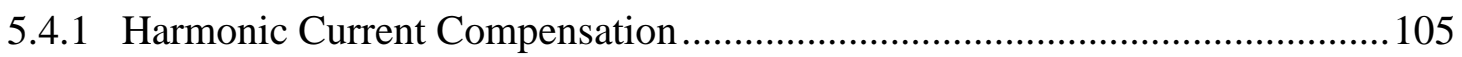

5.4.2 Isolation of Voltage Disturbances ................................................................ 107

5.4.3 Real and Reactive Power Flow Control ..................................................... 108

5.4.3.1 Reactive Power Compensation with SSND during Grid-Connected Steady-State Operation of the MMG System.............................................. 109

5.4.3.2 Combined Real Power Support and Reactive Power Compensation with SSND during Islanding of a Microgrid in the MMG System .................110

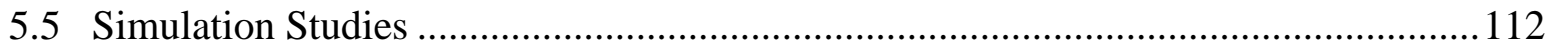

5.5.1 Test Case 1 - Harmonic Current Compensation ............................................. 112

5.5.2 Test Case 2 - Isolation of Voltage Harmonics/Unbalance and Fault-induced

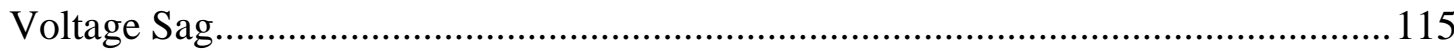

5.5.3 Test Case 3 - SSND Functioning as a Real and Reactive Power Flow Controller

CHAPTER 6 CONCLUSIONS AND RECOMMENDATIONS FOR FUTURE

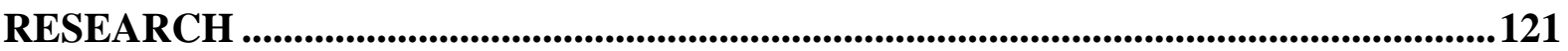

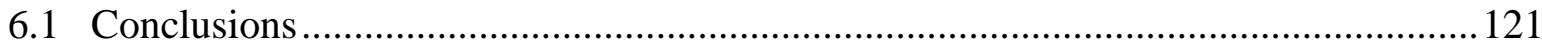

6.2 Recommendations for Future Research ............................................................... 123

REFERENCES........................................................................................................................ 125

APPENDIX A

LIST OF PUBLICATIONS .............................................................................................. 141 


\section{LIST OF FIGURES}

Figure 1-1: A simplified schematic illustration of a microgrid [3] .......................................

Figure 1-2: Control and management architecture of MMG system [1] ...............................

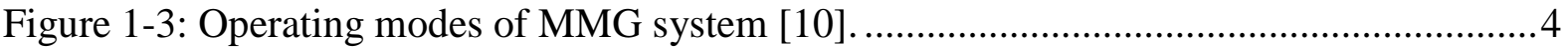

Figure 2-1: Hierarchical control scheme of a MMG system [1] ......................................... 14

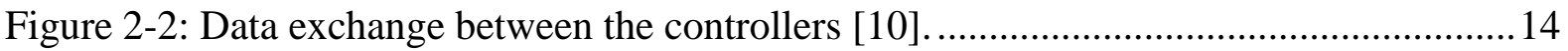

Figure 2-3: Centralized controller must give more weightage to local needs than market participation [1].

Figure 2-4: Hysteresis current control for a single-phase inverter: (a) Control scheme, (b)

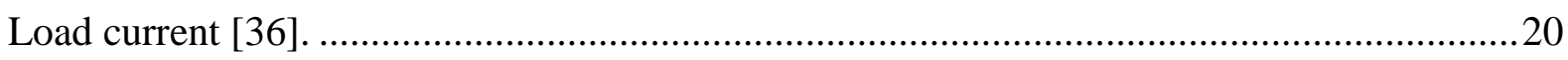

Figure 2-5: Pulse width modulation for a single-phase inverter: (a) Control scheme, (b)

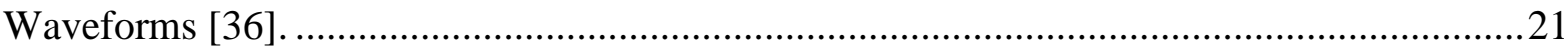

Figure 2-6: Classification of predictive control methods used in power electronics [36]......23

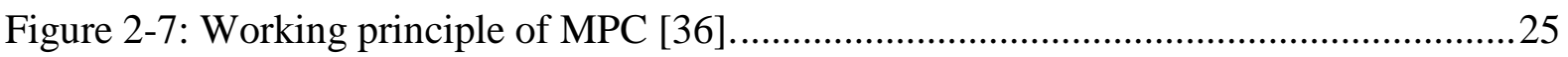

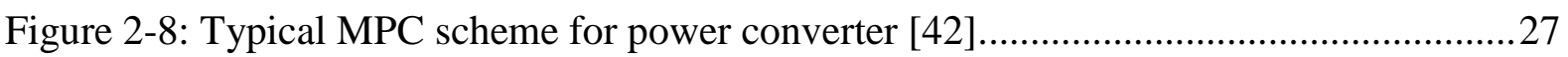

Figure 2-9: Features of MPC that make it an ideal control technique for converters [36] .....28

Figure 2-10: Power transfer from grid to microgrid and vice-versa [51] . .............................29

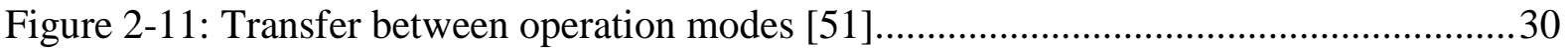

Figure 2-12: Architecture of microgrid under master-slave control mode [51]. .................... 32

Figure 2-13: Architecture of microgrid under peer-to-peer control mode [51] .......................34

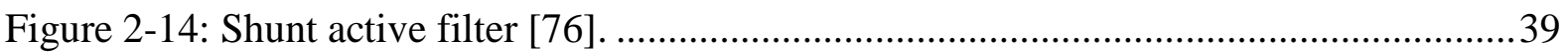

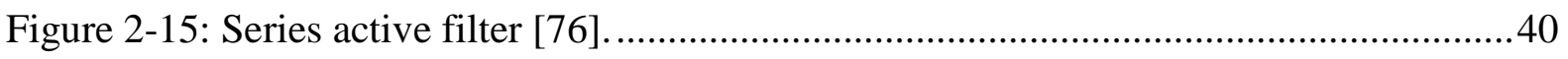

Figure 2-16: Parallel combination of an active filter and a passive filter [76]. ......................41

Figure 2-17: Hybrid topology with series active filter and shunt passive filter connected with

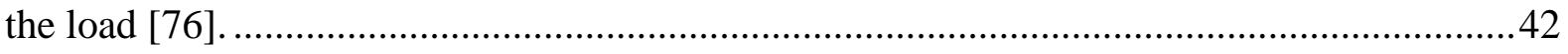

Figure 2-18: Combined topology of series active and passive filters, in parallel with the load [76] .42

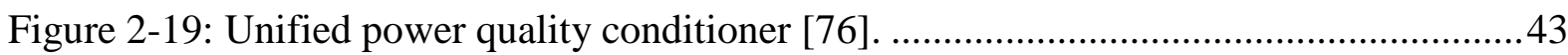

Figure 3-1: Proposed generalized architecture of an AC/AC multi-area MMG system..........47

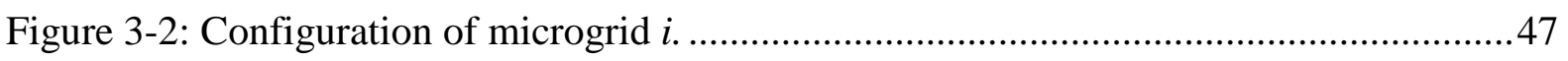

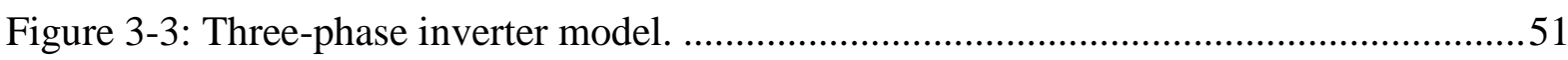

Figure 3-4: Proposed control system for AC/AC multi-area MMG system..........................51

Figure 3-5: Flowchart of the proposed MPC algorithm for voltage control............................55 
Figure 3-6: Flowchart of the proposed MPC algorithm for current control. ..........................55

Figure 3-7: Power flows in the MMG system during steady-state operation.

Figure 3-8: Power flows in the MMG system during islanding of microgrid 2 in test case 1.61

Figure 3-9: Phase angle of load 2 bus voltage.

Figure 3-10: Three-phase load 2 bus voltage for $3.95 \leq \mathrm{t} \leq 4.1 \mathrm{~s}$. 63

Figure 3-11: Real power at the sending and receiving ends of lines 1 and 2.

Figure 3-12: Reactive power at the sending and receiving ends of lines 1 and 2. .63

Figure 3-13: Real power outputs of battery inverters 1, 2 and 3.

Figure 3-14: Reactive power outputs of battery inverters 1, 2 and 3 64

Figure 3-15: Real and reactive power outputs of DG inverters 1, 2 and 3. .64

Figure 3-16: System frequency response. .64

Figure 3-17: Power flows in the MMG system during islanding of microgrid 2 in test case 2.

Figure 3-18: Phase angle of load 2 bus voltage. .66

Figure 3-19: Real power outputs of battery inverters 1, 2 and 3. .66

Figure 3-20: System frequency response. .66

Figure 3-21: Real power consumed by load 2. .66

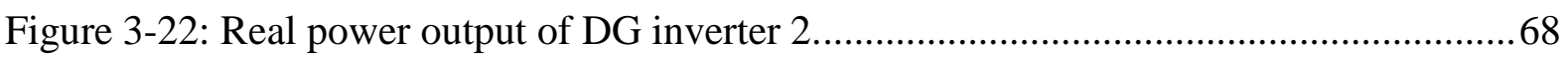

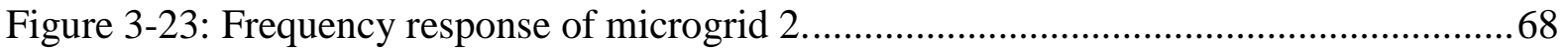

Figure 3-24: Zoom-in frequency response of microgrid 2 for $4 \leq \mathrm{t} \leq 4.9 \mathrm{~s}$..........................68

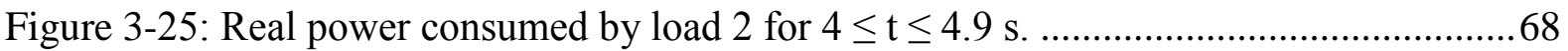

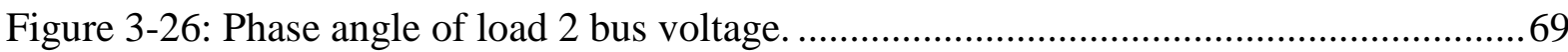

Figure 3-27: Real power flows at the sending and receiving ends of lines 1 and 2...............69

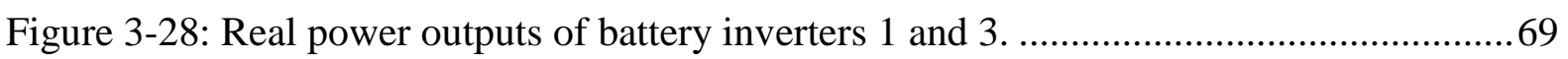

Figure 3-29: Real power output of DG inverter 1 with PI controller. ................................... 71

Figure 3-30: Real power output of DG inverter 1 with MPC controller. …........................... 71

Figure 3-31: Reactive power output of DG inverter 1 with PI controller...............................71

Figure 3-32: Reactive power output of DG inverter 1 with MPC controller.......................... 71

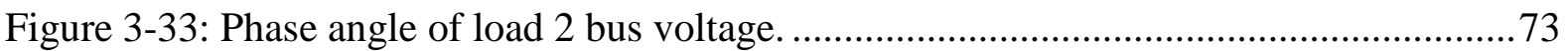

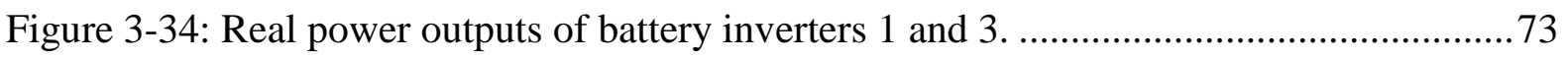

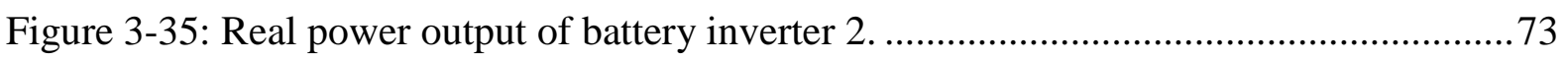

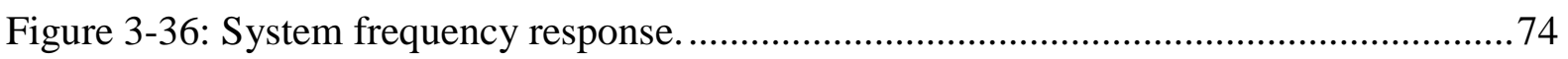

Figure 4-1: Overall Configuration of the proposed AC/DC multi-area MMG system............76 
Figure 4-2: Schematic diagram of microgrids: (a) AC microgrids 1 and 3, (b) DC microgrid 2.

Figure 4-3: Power circuit of AC/DC converter connected to distribution grid 2. ..................78

Figure 4-4: Control scheme for AC/DC converter [36].................................................... 79

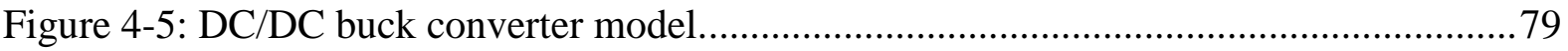

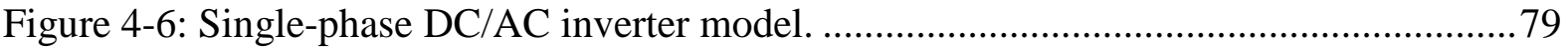

Figure 4-7: Proposed control system for AC/DC multi-area MMG system.......................... 87

Figure 4-8: Power flows in the MMG system during steady-state operation.......................90

Figure 4-9: Power flows in the MMG system during islanding of DC microgrid 2 2..............90

Figure 4-10: Power exchange in the MMG system during load increase in DC microgrid 2. 90

Figure 4-11: Secondary phase voltage at point ' $G$ ' in the MMG system. .............................90

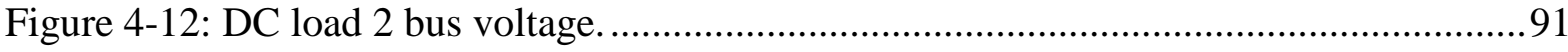

Figure 4-13: Real power output of supercapacitor converter 2c.....................................91

Figure 4-14: Real power output of battery converter 2 b.................................................. 91

Figure 4-15: Three-phase output voltage of the interconnecting inverters 1 and 2..............93

Figure 4-16: Phase angles of output voltages of the interconnecting inverters 1 and 2........93

Figure 4-17: Real powers at the sending and receiving ends of lines 1 and 2.....................93

Figure 4-18: Reactive powers at the sending and receiving ends of lines 1 and 2................94

Figure 4-19: Real power output of supercapacitor converter 2c........................................ 94

Figure 4-20: Real power outputs of battery inverters 1 and 3.........................................94

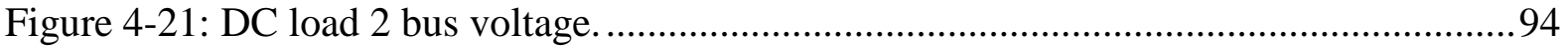

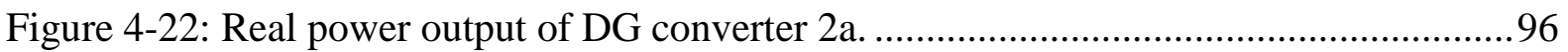

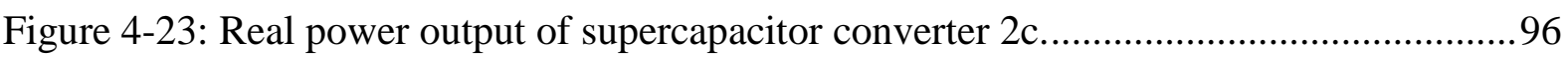

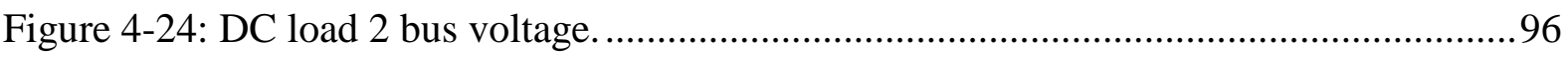

Figure 4-25: Zoom-in DC load 2 bus voltage for $12 \leq \mathrm{t} \leq 12.9 \mathrm{~s}$......................................96

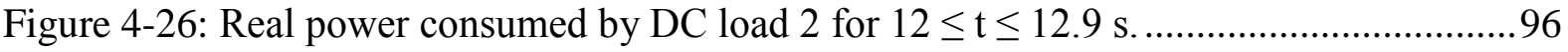

Figure 5-1: Overall configuration of the proposed SSND and MMG system...................... 100

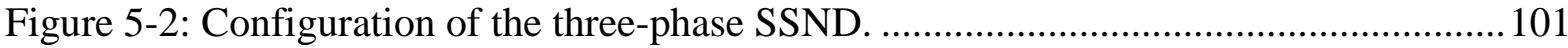

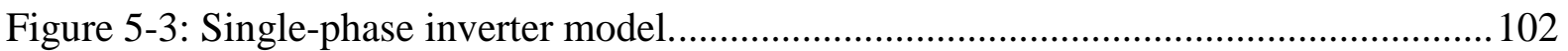

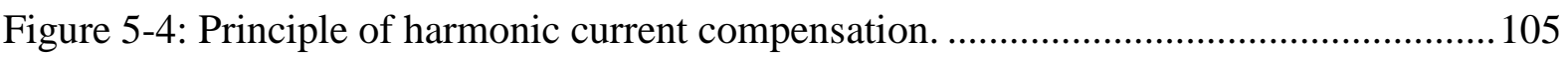

Figure 5-5: Principle of real and reactive power control. ............................................... 108

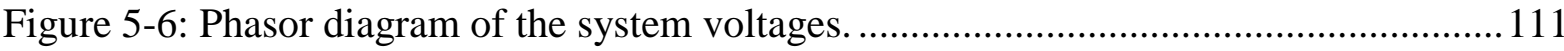

Figure 5-7: Control region of the attainable receiving end real power $P_{R}$ and reactive power $Q_{R}$ with a SSND-controlled line at $\delta=0^{\circ}$ (where c is the center and $\mathrm{r}$ is the radius)............111 


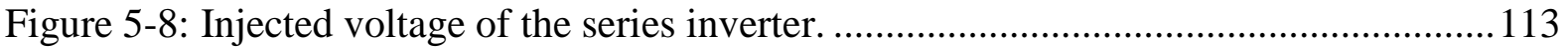

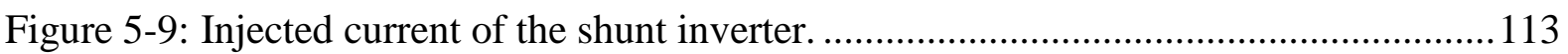

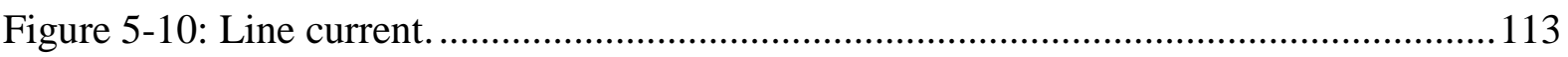

Figure 5-11: Distorted harmonic current in total load 1 (total load 2)................................ 114

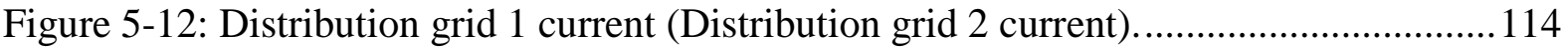

Figure 5-13: Harmonic spectra of total load and distribution grid currents. .........................114

Figure 5-14: Load 1 bus voltage with and without SSND ............................................... 116

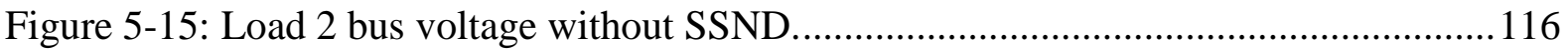

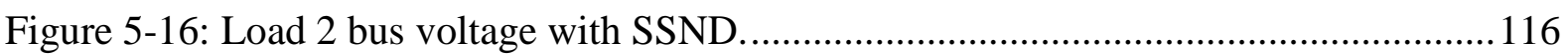

Figure 5-17: Load 1 bus voltage with and without SSND during voltage sags....................116

Figure 5-18: Load 2 bus voltage without SSND.......................................................... 117

Figure 5-19: Load 2 bus voltage with SSND............................................................... 117

Figure 5-20: Reactive power supplied by the shunt inverter and reactive power at the

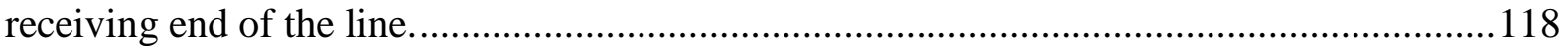

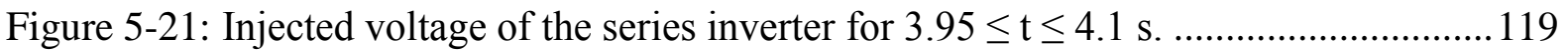

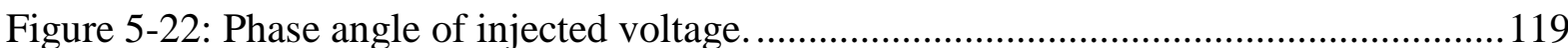

Figure 5-23: Real powers supplied by DG inverters 1 and 2, and shunt inverter and real power at the receiving end of the line.

Figure 5-24: Real and reactive power delivered by distribution grids 1 and 2 during steady

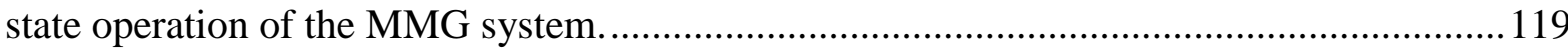

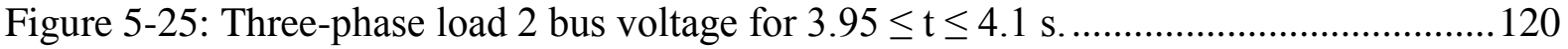

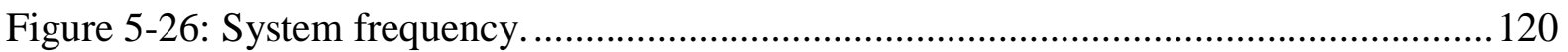

Figure A-1: Single-phase equivalent circuit of the MMG system..................................... 136 


\section{LIST OF TABLES}

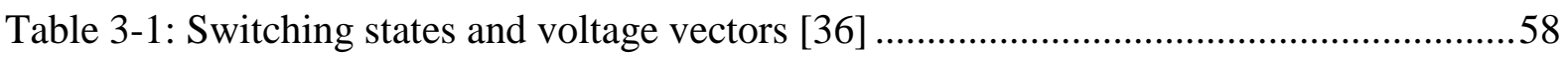

Table 3-2: Parameters of the proposed MMG system .......................................................59

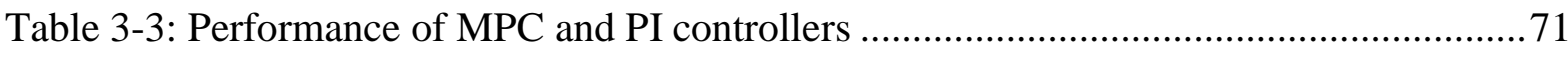

Table 3-4: Performance of MPC and PI controllers in power reference tracking for test case 2 .73

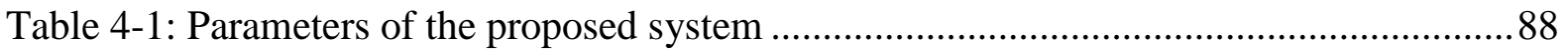

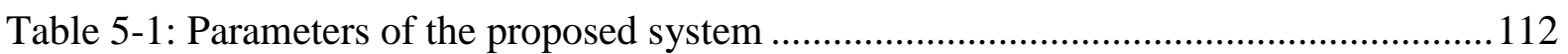

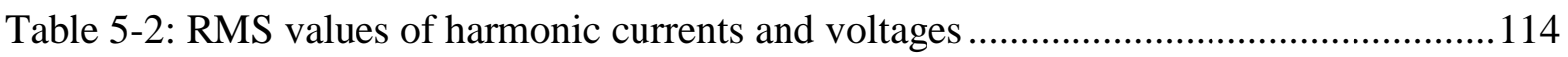

Table 5-3: Harmonic spectra of distribution grid 1 voltage .............................................. 115 


\section{LIST OF ABBREVIATIONS}

$\mathrm{AC}$

$\mathrm{AC} / \mathrm{AC}$

$\mathrm{AC} / \mathrm{DC}$

AGC

APF

APLC

CAMC

$\mathrm{CCM}$

DC

DG

DMS

DVR

ES

FFT

HV

IEEE

IET

LV

MAS

$\mathrm{MC}$

MGCC

MMG

MPC

MV

OLTC

PI

PQ

PV

PWM

RMS

RTU

SOC
Alternating Current

Interconnection of one $\mathrm{AC}$ microgrid with another $\mathrm{AC}$ microgrid

Interconnection of one $\mathrm{AC}$ microgrid with another $\mathrm{DC}$ microgrid

Automatic Generation Control

Active Power Filter

Active Power Line Conditioner

Central Autonomous Management Controller

Current Control Mode

Direct Current

Distributed Generation

Distribution Management System

Dynamic Voltage Restorer

Energy Storage

Fast Fourier Transform

High-Voltage

Institute of Electrical and Electronics Engineers

Institution of Engineering and Technology

Low-Voltage

Multi-Agent System

Microsource Controller

Microgrid Central Controller

Multi-Microgrid

Model Predictive Control

Medium Voltage

On-Load Tap Changer

Proportional-Integral

Power Quality

Photovoltaic

Pulse Width Modulation

Root Mean Square

Remote Terminal Unit

State of Charge 
SSND

SVC

THD

UPQC

VCM
Series-Shunt Network Device

Static VAR Compensator

Total Harmonic Distortion

Unified Power Quality Conditioner

Voltage Control Mode 


\section{CHAPTER 1 INTRODUCTION}

This chapter presents a brief description about the basic concept, control and management architecture, operating modes and various benefits of multi-microgrid (MMG) system. In addition, research motivations, objectives, contributions and organization of this thesis are described in this chapter.

\subsection{Introduction to MMG System}

The concept of a MMG system consists of a high level structure formed at the medium voltage (MV) level, comprising several interconnected microgrids [1]. A MMG system consists of AC or DC microgrids or a combination of both AC and DC microgrids. Basically, a microgrid is defined as a low-voltage (LV) distribution system with distributed generations (DGs) such as solar photovoltaics (PVs), microturbines, wind turbines, and fuel cells together with storage devices (battery banks or flywheels) and flexible loads [2]. A simplified schematic illustration of a microgrid is shown in Figure 1-1 [3]. A microgrid has two modes of operation namely grid-connected mode and islanded mode where the microgrid is disconnected from the grid [4], [5]. A microgrid differs from a traditional utility grid mainly in terms of the management and coordination of available resources [1]. Another one of the major differences between a microgrid and a utility grid is that a microgrid, unlike a utility grid, is dominated by inverter based DG units and so operation and protection of DG units must be suited to power electronic technologies. Power electronics control plays a major role in interfacing the DG units to the microgrid [3].

There has been a large increase in the number of microgrid installations all over the world in recent years. In the future more and more microgrids will be interconnected to form large multi-microgrid systems to meet the ever increasing global energy demands. Interconnection of microgrids is seen as a viable solution for the massive integration of renewable energy systems as envisaged by the supergrid concept proposed by Desertec foundation [6].

The differences between a MMG and a utility grid are as follows:

a) DG units in a MMG system are of much smaller capacity with respect to the large generators in a utility grid. 
b) DG units are normally installed close to the electrical loads in a MMG system whereas in a utility grid, the sources of generation are usually located far away from the customer's loads.

c) MMG system, unlike the utility grid, is dominated by renewable energy resources and inverter based DG units and thus the operation, control and protection of DG units must be suited to power electronic technologies.

d) MMG system is a distribution system formed at the medium-voltage (MV) level in a utility grid and it does not operate at high-voltage (HV) level. But a utility grid consists of a HV transmission system in addition to a HV generation system and a MV distribution system.

A single microgrid system can only produce and distribute power within a localized area. In addition, the intermittent power generated by renewable energy sources such as solar PV often causes variation in the power, voltage and frequency of the system [7]. Furthermore, in the event of islanding of a microgrid from the grid, a single microgrid system itself may not be reliable and secure enough to meet its own load demand due to its restricted energy generation capability [7], [8]. In such cases, the single microgrid system usually employs load shedding technique to ensure supply-demand balance which is usually inconvenient and economically undesirable as it poses the risk of causing disruption to customers' business operations [7]. To overcome these challenges in a single microgrid system and ensure improved reliability and security of power supply, different architectures of MMG systems consisting of interconnected microgrids are proposed in this thesis. The main significance of MMG systems is that multiple microgrids in adjacent locations can be interconnected to meet larger bulk power demands and these microgrids can mutually exchange power among each other to satisfy their load demands, during grid-connected and islanded modes of operation [7].

An effective management of the MMG system requires the development of hierarchical control architecture as illustrated in Figure 1-2 [1], [9]. Distribution management system (DMS) is in charge of the medium-voltage (MV) distribution network [1]. The control of the overall MMG system is exercised by a controller called central autonomous management controller (CAMC), which is installed at the MV bus level of a HV/MV substation [1]. CAMC manages the microgrid central controller (MGCC), which controls each individual microgrid, consisting of DG units [1]. In this way, the complexity of the MMG system can be shared among several smaller individual control agents [9]. 
In order to tackle the scheduling problem of DG units and other control devices that are installed in the system in both normal and emergency conditions, there is an essential need to deploy a smart metering infrastructure, which allows a coordinated management of individual elements at the LV level, such as microgrids (and corresponding DG units, battery units and loads) [10]. Remote terminal unit (RTU) is a device used to collect the required information such as load demands in the microgrids, transformer tap-settings, feeder power flows, etc and send this information to CAMC. DMS is the highest control level, which is in charge of multiple microgrids. The detailed explanation of the hierarchical control scheme and the different controllers such as CAMC and MGCC is given in Chapter 2.

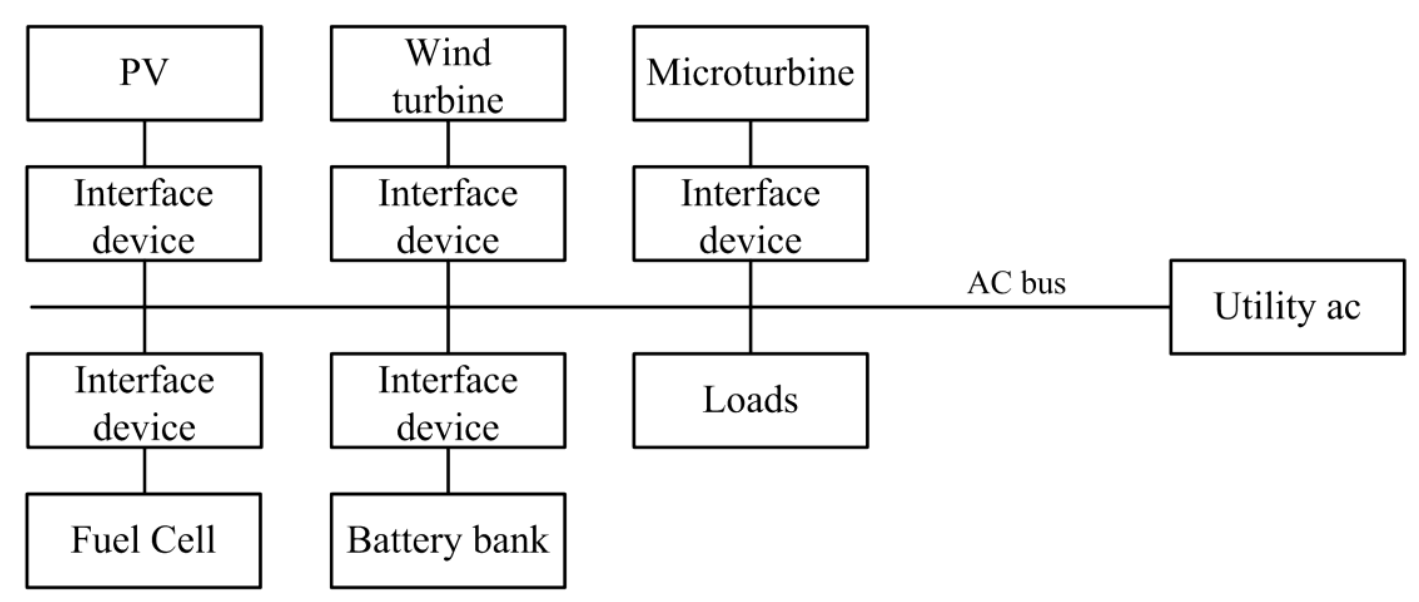

Figure 1-1: A simplified schematic illustration of a microgrid [3].

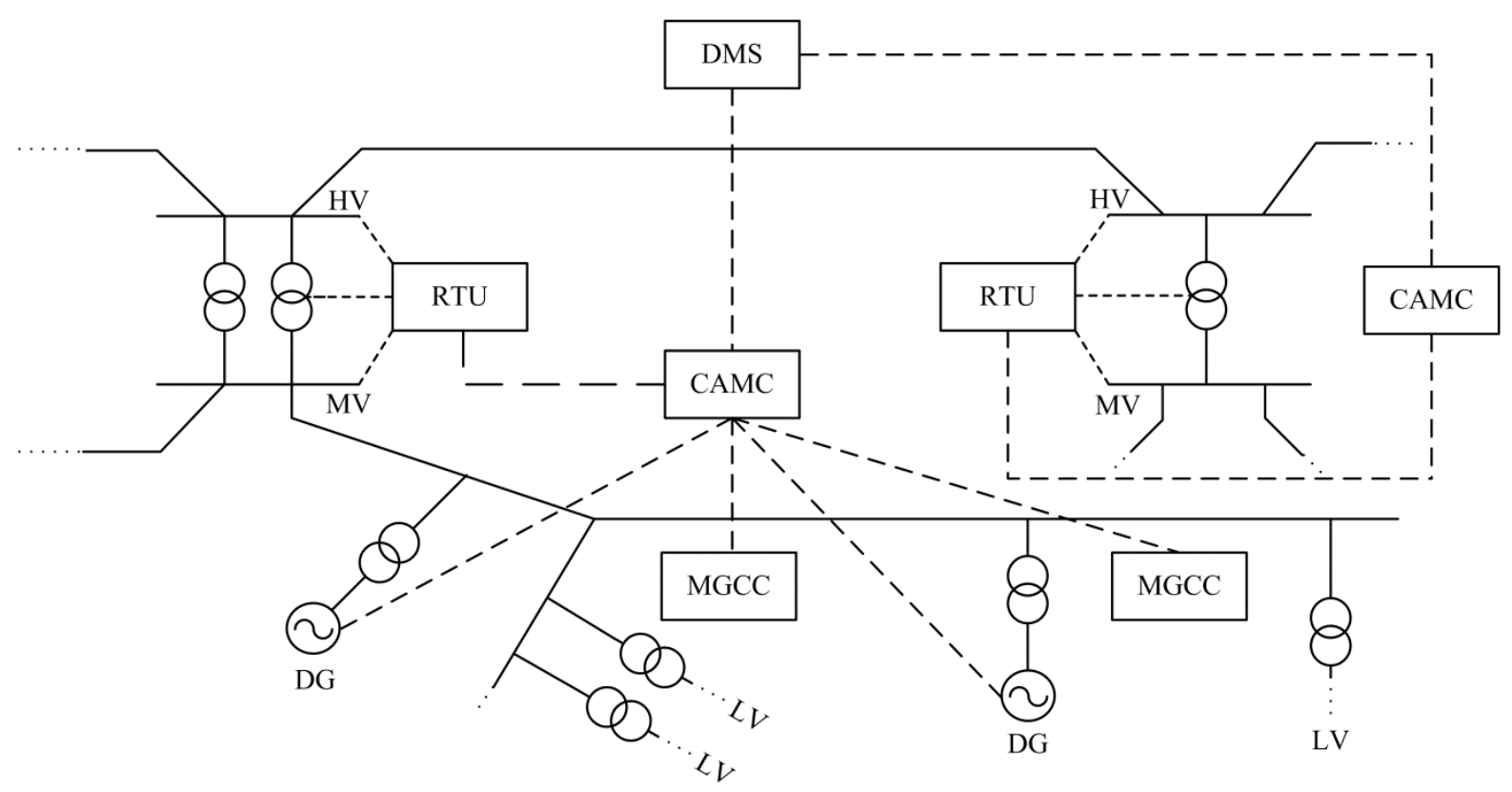

Figure 1-2: Control and management architecture of MMG system [1]. 


\subsection{Operating Modes of MMG System}

A MMG system basically operates in grid-connected mode, when it is connected to the MV distribution grid and also operates in an autonomous or islanded mode, when it is disconnected from the MV distribution grid [10]-[12].

The five different operating modes of the MMG system are shown in Figure 1-3 and are explained as follows [10]:

- Interconnected mode - In this mode, the MMG system is connected to the distribution grid and the distribution grid regulates the voltage and frequency of the MMG system.

- Islanded mode - MMG system with an effective control system operates autonomously when disconnected from the distribution grid and it regulates the load voltage and system frequency itself to ensure uninterrupted power supply to its loads.

- Synchronization - MMG system after islanding remains in this mode until reconnection with distribution grid occurs. In this mode, the islanded MMG system is synchronized by making its frequency, voltage and phase sequence same as that of the distribution grid and the islanded MMG system is prepared for reconnection to the distribution grid.

- System collapse - Here the whole MMG system totally experiences a black out due to the occurrence of a single or multiple contingencies and all the DG units are shut down.

- Black start - In this mode, the MMG system is slowly restored from the state of system collapse, with re-connection of DG units initially followed by the formation of small islands.

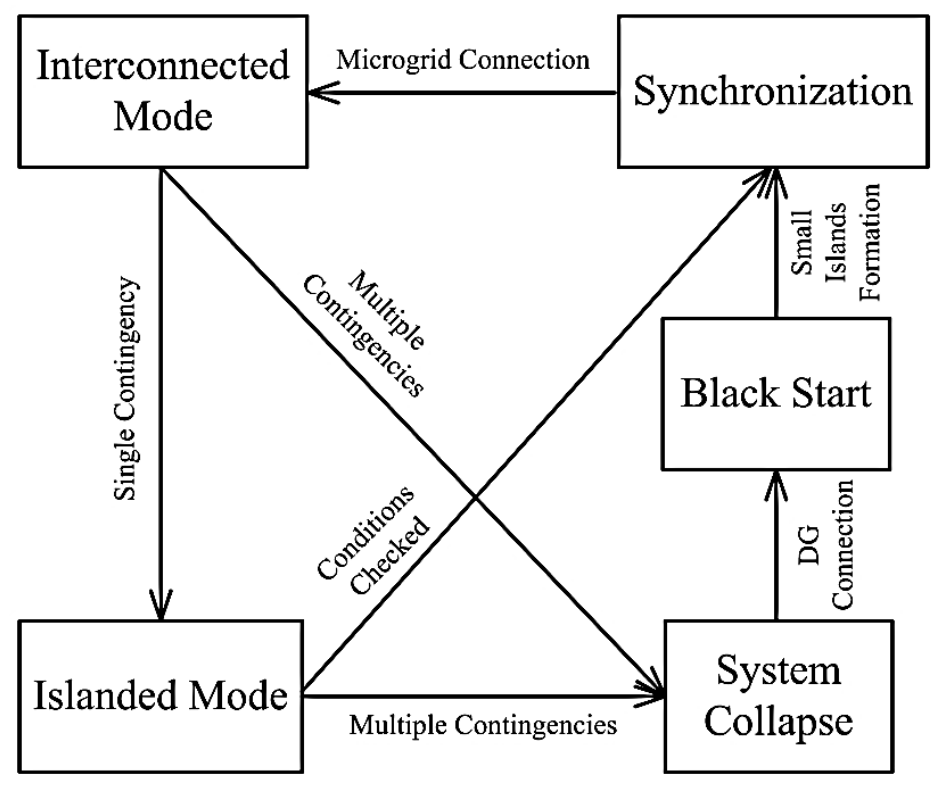

Figure 1-3: Operating modes of MMG system [10]. 


\subsection{Advantages of MMG System}

There are several advantages associated with interconnection of microgrid systems in the MMG system which are briefly described as follows:

- Improving reliability and sharing power reserves: The amount of reserve capacity that must be built by an individual microgrid system to ensure reliable operation when power shortage occurs can be reduced by sharing reserves within an interconnected microgrid system [13].

- Reduced investment cost in generating capacity: Individual microgrid systems can reduce their generating capacity requirement, or need not add new capacity, if they are able to share the generating resources of an interconnected microgrid system [13].

- Increasing load diversity and improving load factor: Microgrid systems operate most economically when the level of power demand is steady over time [13]. There is also a need to maintain an economical operation of microgrid systems during peak and off-peak hours of the distribution grid [13]. Poor load factors (the ratio of average to peak power demand) mean that microgrid systems must construct generation capacity to meet peak requirements, but that this capacity sits idle much of the time [13]. Microgrid systems can meet the peak power requirements and improve the load factors by interconnecting to other microgrid systems with different types of loads with different daily or seasonal patterns that complement their own [13].

- Economies of scale in new construction: The unit costs of new generation, transmission and distribution capacity in the interconnected microgrids generally decrease with increasing scale of the MMG system [13]. Sharing resources in the MMG system can allow the construction of larger generation sources with lower unit costs [13].

- Supply security and diversity of generation mix: Interconnection between microgrid systems that use different technologies to generate electricity provides greater security in the event that one kind of generation becomes limited (e.g., solar energy during night) [13]. This complementarity can be a strong incentive for interconnection between PV dominated systems and fuel cell dominated systems, thus improving reliability and security of power supply [13].

- Improving microgrid resilience: Due to major disturbance at the utility side, microgrids may encounter emergencies such as unintentional islanding and load shedding [14]. 
Interconnection of microgrids mitigates the impact of such emergencies and thus improves the resilience of microgrids [14].

- Economic exchange: Interconnection of microgrids allows the dispatch of the least costly distributed generating units within the interconnected area, providing an overall cost savings that can be divided among the individual microgrid systems [13]. Alternatively, it allows inexpensive power from one microgrid system to be sold to microgrid systems with more expensive power [13].

\subsection{Research Motivations}

There has been an increase in the use of renewable energy sources such as PV, wind, tidal wave, and hydro power in recent years to reduce the greenhouse gas emissions and avoid the depletion of fossil fuels, leading to the emergence of microgrids. Microgrids play an important role in integrating the renewable energy sources in the power grid and in increasing the utilization of these resources. As the global renewable energy demand increases every year, more and more microgrids need to be installed in different parts of the world.

To achieve the widespread usage of microgrids, and improve the reliability and security of microgrids, interconnection of microgrids forms the next strategic step in the massive largescale integration of renewable energy resources into the power grid. A single microgrid has limited energy generation capacity and will need to resort to load shedding in case of a power shortage during islanding. But if several microgrids are interconnected to form MMG systems, this will enable sharing of power reserves among the interconnected microgrids during emergency conditions, and thus improve the resilience and minimize the load shedding requirements of individual microgrid systems during islanded situations [14].

Considering these benefits and future potential of the MMG systems, different types of multiarea MMG systems such as AC/AC multi-area MMG system and AC/DC multi-area MMG system are proposed in this thesis. The operation and control of these multi-area MMG systems remains a challenging problem due to complexity and scale involved in such largescale interconnected renewable energy systems. Thus, the main motivation of this thesis is to solve this challenging problem and develop efficient control strategies and algorithms to facilitate accurate and desired power exchange among the interconnected microgrids in the proposed MMG systems. Furthermore, power quality issues such as harmonic currents, 
voltage disturbances and low power factor are investigated in these MMG systems. Then a device called series-shunt network device (SSND) is developed in this thesis to solve the power quality issues in these MMG systems. In general, the proposed control strategies and power quality improvement schemes for the multi-area MMG systems should contribute to the advancement of renewable energy technologies and promotion of the wide-scale interconnection of microgrid systems to achieve global energy sufficiency.

\subsection{Objectives of Thesis}

Considering the motivations of this research, the main focus of this research is the development of effective control strategies and improvement of power quality in multi-area MMG systems. The detailed objectives of this thesis are summarized as follows:

1) The first objective is to develop a generalized architecture of an AC/AC multi-area MMG system, consisting of interconnected AC microgrids. In this MMG system, effective control systems need to be developed for controlling the power exchanges among interconnected microgrids. When a microgrid in this MMG system is islanded from the distribution grid, the load voltage and system frequency of the islanded microgrid are affected. Therefore, a control system is to be developed in this thesis for solving this problem of voltage and frequency variations and for facilitating an effective mutual power exchange among the interconnected microgrids. In addition, an improved model predictive control (MPC) algorithm is to be developed for the control of multiple inverters to achieve better control performance in the proposed MMG system.

2) The second objective is to develop an efficient control scheme for regulating power flows and load voltages in an AC/DC multi-area MMG system, consisting of interconnected AC and DC microgrids. With the emergence of DC microgrids in recent years and the increasing need for interconnection of AC and DC microgrids, there arises a necessity for developing reliable control schemes for the coordinated operation and control of interconnected AC and DC microgrid systems. In view of this necessity, an efficient control system is to be developed in this thesis to control the power exchanges among the interconnected AC and DC microgrids and to regulate the load bus voltages, especially during microgrid islanding. Furthermore, a state-space model based MPC, which is independent of the system 
parameters, is required to be used for controlling multiple inverters and converters in the AC/DC multi-area MMG system.

3) The final objective of this thesis is to investigate power quality issues and propose new methods of power quality improvement in the AC/AC multi-area MMG system. A new method of non-local harmonic current and reactive power compensation, voltage disturbance isolation and real power support is to be implemented in the AC/AC multi-area MMG system using a device called SSND. In addition, several power quality and power flow control functions of SSND are to be analyzed and verified in grid-connected and islanded modes of the interconnected microgrids in the AC/AC multi-area MMG system.

\subsection{Contributions of Thesis}

The main contributions of this thesis are summarized as follows:

1) In this thesis, a generalized architecture of an AC/AC multi-area MMG system is proposed. It consists of several AC microgrids separated from each other by interconnecting lines. Each microgrid in this MMG system consists of a DG unit, a storage battery unit and an AC load. The DG and storage battery units are connected to the AC load bus through their inverters. To efficiently share the load demand among these inverters and to facilitate effective power exchange among the interconnected microgrids, a control system consisting of centralized and local controllers is proposed for the AC/AC multi-area MMG system. In addition, an improved MPC algorithm which has the capability to minimize the control error and achieve better control performance during the islanding of a microgrid in the MMG system is proposed for the local control of DG and storage battery inverters. The thesis aims to provide a comprehensive solution specifically for the problem of voltage and frequency variations during islanding of a microgrid in an AC/AC multi-area MMG system using the proposed MPC algorithm. Several simulation studies are also conducted to validate the performance of the AC/AC multi-area MMG system in achieving effective voltage and frequency regulation under different operating modes such as grid-connected and islanded modes of the interconnected AC microgrids.

2) This thesis proposes an overall configuration for an AC/DC multi-area MMG system. It consists of both AC and DC microgrids separated from each other by interconnecting lines. The AC microgrid consists of a DG unit, a storage battery unit and an AC load. In the AC 
microgrid, the DG and the storage battery units are connected to the AC load bus through their inverters. The DC microgrid consists of a DG unit, a storage battery unit, a supercapacitor unit and a DC load. The DG, storage battery and supercapactor units are connected to the DC load bus through their DC/DC buck converters. The DC microgrid is interconnected with other AC microgrids through interconnecting inverters and lines. To ensure accurate power sharing among various inverters and converters in each microgrid and achieve effective power exchange between the AC and DC microgrids, state-space model based MPC algorithms are developed for the control of inverters and converters in the proposed AC/DC multi-area MMG system. In addition, a control system consisting of centralized controller and local MPC controllers is developed to solve the problems of power imbalance and DC voltage variation during different operating conditions of the interconnected DC microgrid such as islanding, load increase and load shedding in the proposed AC/DC multi-area MMG system. The performance of the AC/DC multi-area MMG system in effective voltage and power regulation is also verified under different operating conditions with several simulation studies.

3) In this thesis, a new method of power quality improvement is developed for the AC/AC multi-area MMG system. Power quality issues such as harmonic current distortion, voltage sag and low power factor are explored in the proposed AC/AC multi-area MMG system. Then a new method of non-local harmonic current and reactive power compensation, voltage disturbance isolation, and emergency active power support with a device called SSND is proposed for the AC/AC multi-area MMG system. In this non-local compensation method, SSND can be installed at some distance away from the loads and need not necessarily be installed very close to the loads, whereas in local compensation, the compensating device must always be placed very close to the loads. The power quality and power flow control functions of SSND are analyzed during grid-connected and islanded modes of the interconnected AC microgrids. A state-space model based MPC algorithm is applied for the control of multiple DG inverters, series and shunt inverters of SSND in the AC/AC multiarea MMG system. The proposed SSND is capable of accomplishing the following tasks in the AC/AC multi-area MMG system:

- Local and non-local harmonic current and reactive power compensation

- Isolation of one microgrid from voltage disturbances such as voltage distortion, voltage sag and voltage unbalance occurring in adjacent microgrid

- Real power support during islanding of a microgrid 


\subsection{Organization of Thesis}

The thesis is organized into six main chapters as follows:

\section{Chapter 1: Introduction}

This chapter introduces the basic concept of MMG system. In this chapter, the control and management architecture, operating modes and benefits of MMG system are briefly discussed. It also outlines the research motivations, objectives, contributions and organization of the thesis.

\section{Chapter 2: Literature Review}

In this chapter, a comprehensive literature review on the different hierarchical control schemes and advantages of the centralized scheme used in this thesis are explained in detail. Different types of control schemes of DG units including the proposed MPC are discussed. The advantages of using MPC over conventional control schemes in the MMG systems are specifically emphasized. The typical power quality issues and the power quality improvement methods used are also reviewed. In addition, a multi-area MMG system is defined and the different types of multi-area MMG systems, namely AC/AC multi-area MMG system and AC/DC multi-area MMG system are presented.

\section{Chapter 3: Operation and Control of AC/AC Multi-Area MMG System}

Chapter 3 presents a generalized architecture of an AC/AC multi-area MMG system, consisting of interconnected AC microgrids. In this chapter, a control system and an improved MPC algorithm are proposed for the control of multiple DG and storage battery units in the proposed AC/AC multi-area MMG system. The aim of this chapter is to solve the voltage and frequency variations which occur during the microgrid islanding in the proposed AC/AC multi-area MMG system using the developed control system and MPC algorithm. Several simulation studies are conducted to verify the control performance in effective voltage and frequency regulation especially during microgrid islanding and load shedding in the AC/AC multi-area MMG system. 


\section{Chapter 4: Voltage and Power Control in an AC/DC Multi-Area MMG System}

In Chapter 4, a control scheme is developed for another type of multi-area MMG system, which is AC/DC multi-area MMG system. This control scheme is used to facilitate desired power exchange among the interconnected AC and DC microgrids especially during the islanding of AC or DC microgrid and is also useful in solving the problem of DC bus voltage variation when the DC microgrid is islanded from the distribution grid. In addition, statespace model based MPC algorithms are proposed to control multiple converters and inverters in the proposed AC/DC multi-area MMG system. From the simulation studies conducted, it is verified that the proposed control algorithms yield desired results in effective power interchange and voltage regulation in the AC/DC multi-area MMG system.

\section{Chapter 5: Series-Shunt Network Device for AC/AC Multi-Area MMG System}

Chapter 5 proposes a new method of solving various power quality issues such as harmonic current distortion, voltage disturbance and low power factor in the proposed AC/AC multiarea MMG system using a device called SSND. The power quality and power flow control functions of SSND are analyzed and SSND is then tested for its power quality improvement capability. Different simulation studies are conducted to verify the effective control of SSND using a state-space model based MPC algorithm and to validate the successful implementation of the new method of non-local harmonic current and reactive power compensation in the proposed AC/AC multi-area MMG system.

\section{Chapter 6: Conclusions and Recommendations for Future Research}

In Chapter 6, an overall conclusion for the thesis is presented. In addition, prospects and recommendations for future research are proposed as well. 


\section{CHAPTER 2 LITERATURE REVIEW}

\subsection{Introduction}

With rapid demand for renewable energy sources and increasing concern for environmental protection, MMG systems are gaining prominence in the power sector today. The development of appropriate control, conversion and power quality improvement schemes is thus crucial for these MMG systems. This chapter briefly reviews the existing hierarchical control scheme of conventional MMG systems. The centralized and decentralized control schemes in MMG systems are evaluated and justifications for using centralized control scheme for the proposed MMG systems are provided. Then, different control techniques used for the DG and storage battery units in the interconnected microgrids of the MMG systems are discussed. The significant benefits of using the proposed MPC algorithm over other conventional MPC algorithms and control methods are also briefly examined. In addition, the operating and control modes of the interconnected microgrids in the MMG systems are reviewed. Moreover, the details about different conventional MMG systems available in literature are provided. Finally, power quality issues in the existing power system and power quality improvement methods currently adopted are explained.

\subsection{Hierarchical Control in the MMG System}

There is an essential need to define control issues and strategies when dealing with MMG systems because of complexity and dimensionality of such systems [1]. Different control schemes such as centralized and decentralized schemes exist for MMG systems [1]. In a centralized controller all the information regarding the DG units, storage battery units and the control actions are gathered and not executed by a centralized controller. On the basis of this information, the centralized controller sends the reference set-points to the low-level local controllers of the DG and storage battery units. But, in a decentralized controller, the control actions are mutually shared among individual local controllers of DG units or microsources and loads [9].

Generally, a hierarchical structure is followed in the control strategy of the MMG system [9]. In this hierarchical strategy, a centralized controller collects all the data from multiple devices in MMG system and delegates tasks to low-level local controllers [9]. In a decentralized scheme, even though the centralized controller does not have to carry out the overall 
management task, the decision making in a decentralized control scheme should still adhere to a hierarchical structure [10]. The hierarchical control scheme of a MMG system is shown in Figure 2-1. The explanation of the three control levels in a MMG system is given as follows [1], [9]:

\section{Control Level 1 - DMS}

DMS is in charge of the entire MV distribution network and controls the interactions between the MV distribution grid and the MMG system. The architecture of the MMG system is already shown in Figure 1-2.

\section{- Control Level 2 - CAMC}

CAMC is in charge of the whole MMG system. It is responsible for data collection for enabling dialogue with DMS located upstream. CAMC performs the task of scheduling generation in order to satisfy the load demand by sending desired power dispatch commands to MGCC, local DG and load units. CAMC is not required to know the details of each microgrid component since MGCC acts as an interface between CAMC and microgrid. In grid-connected mode of the MMG system, all the microgrids are connected to the grid and the autonomous intervention of CAMC is kept to a minimum. Also, in grid-connected mode of the MMG system, CAMC is responsible for economic allocation of various generation units to minimize the overall operating cost similar to economic dispatch function in conventional power systems.

In islanded mode of the MMG system, CAMC sends commands to MGCC and lower level local controllers including microsource controller (MC) and load controller (LC) to modify the power outputs of DG and storage battery units and thus CAMC restores system frequency similar to that performed in convention automatic generation control (AGC) functionality. CAMC also collects information regarding on-load tap changer (OLTC) settings, control modes of static VAR compensator (SVC), DG unit power outputs and load demands in each microgrid. Then CAMC processes this information and decides the appropriate control actions to be sent to MGCC.

\section{- Control Level 3 - MGCC, MC and LC}

MGCC receives instructions from CAMC and sends desired power set-points to lower level local controllers including MC and LC. Generation can be curtailed by MGCC and MC, if excess power imbalance occurs due to variability of intermittent sources such as PV and wind. Similarly, load can be shed by MGCC and LC, if power deficit occurs in the microgrid. 


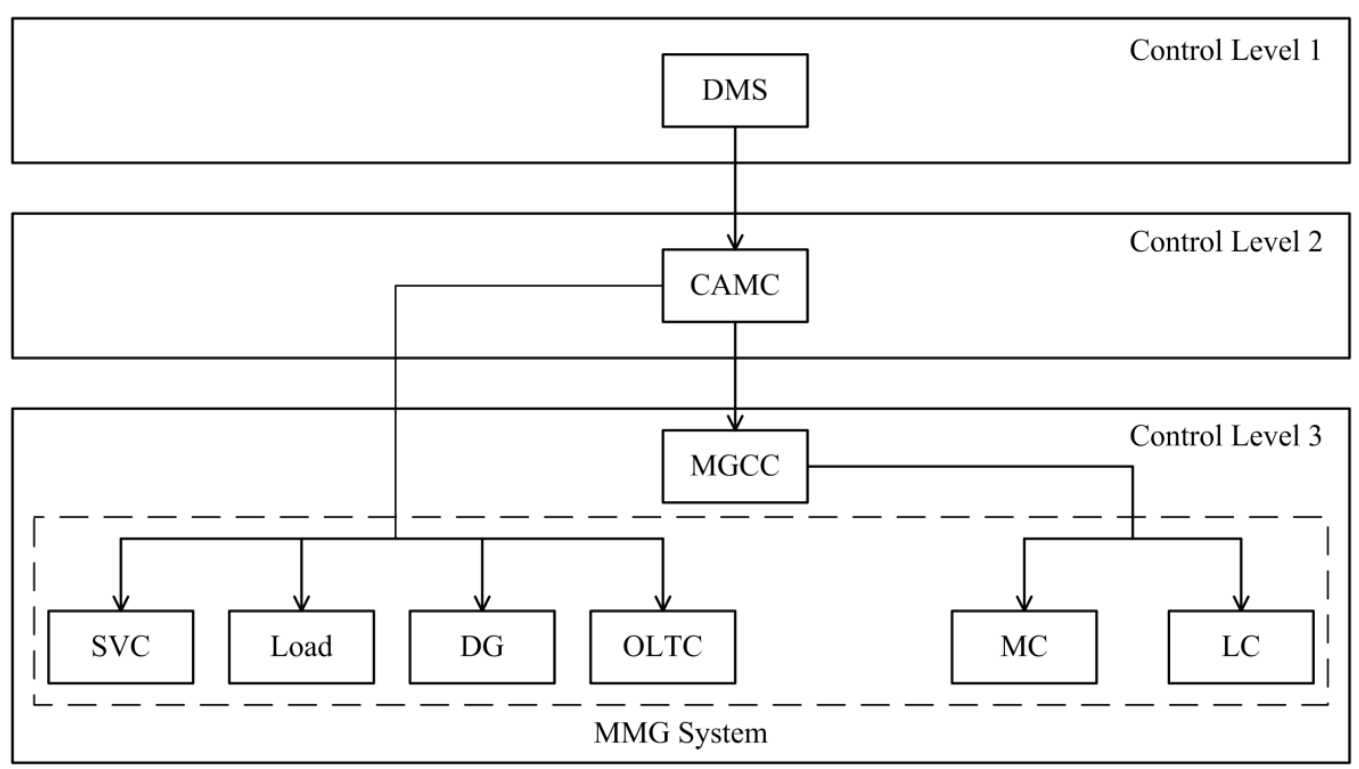

Figure 2-1: Hierarchical control scheme of a MMG system [1].

\subsubsection{Data Exchange among Different Controllers}

The interactions and data exchange among CAMC, MGCC, MC and LC are shown in Figure 2-2. CAMC receives the information about the generation requirements and load demands in each microgrid from MGCC, MC and LC [10]. Then CAMC determines the set-points to be sent back to MGCC, MC and LC [10]. MC sends bidding offers to CAMC for increasing or decreasing production. MGCC and LC send bidding offers to CAMC for increasing or decreasing the load demand.

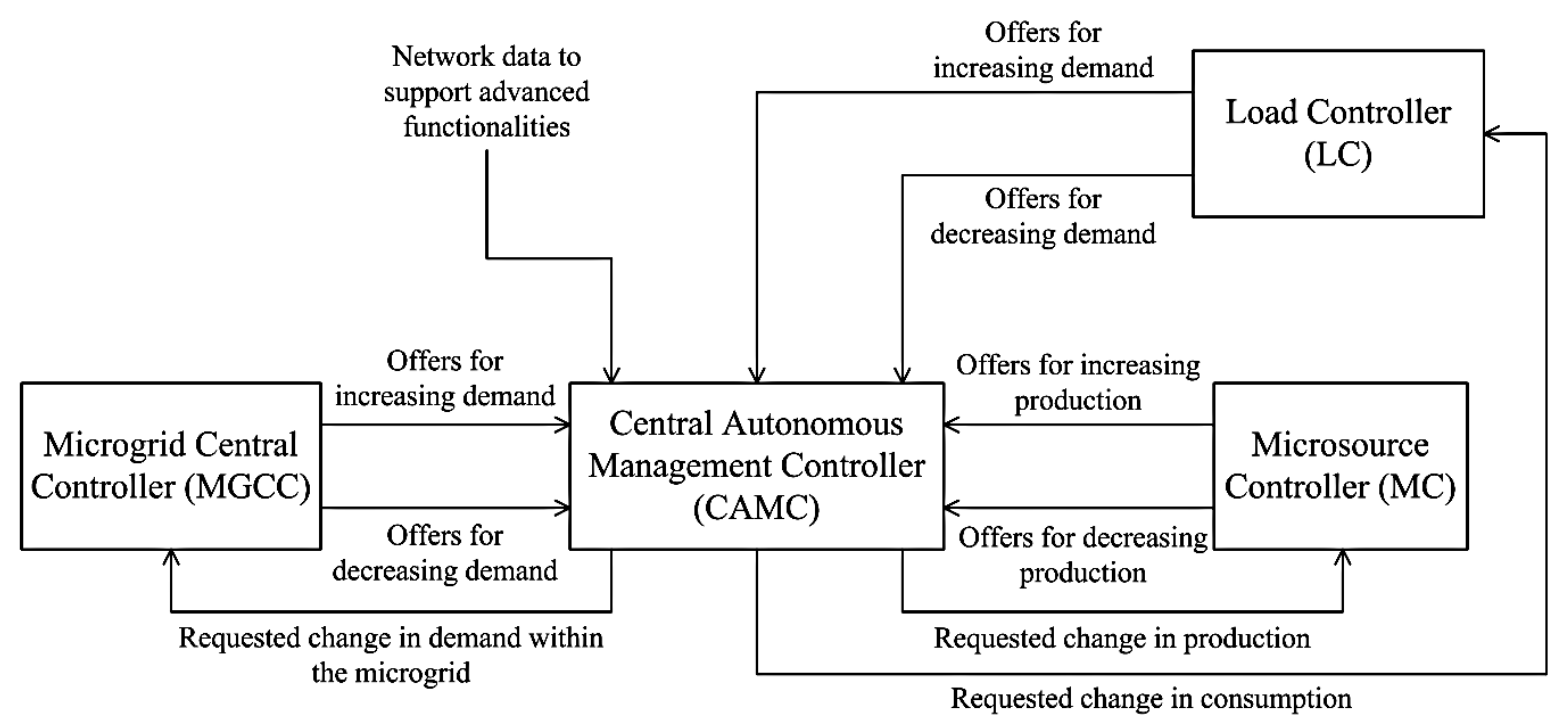

Figure 2-2: Data exchange between the controllers [10]. 


\subsection{Centralized and Decentralized Control Schemes in the MMG System}

Depending on the functions performed by different control levels, the MMG system can be operated based on centralized or decentralized scheme [1].

\subsubsection{Centralized Control Scheme}

In a centralized control scheme, the main objective of control and optimization of the MMG system lies with the CAMC [1]. CAMC monitors the electricity market prices and determines how much power is required by different loads, how much power should be drawn from each DG unit and storage battery unit and how much load is to be shed [9]. In this way, CAMC optimizes the operation of the MMG system in the most economical and profitable way [9], [10].

In a single microgrid, MGCC is the central controller [1]. MGCC collects information such as load demand, production capacity of DG units, state-of-charge (SOC) of storage battery units, solar irradiation, wind speed, etc. [9]. Using this information, MGCC runs optimization algorithm to determine the optimal operation based on chosen objectives [10]. MGCC has the function of sending the required power set-points to DG and storage battery units according to the load demand in microgrids [11]. Thus the basic feature of CAMC is the overall optimal operation of the MMG system and the fundamental objective of MGCC is the optimal operation of the microgrid system [10]. Generally, two market services are identified for the MMG system as follows [1]:

1. The MMG system can cater to its own needs or load demands and try to minimize power drawn from the grid.

2. MMG participates in the energy market through an aggregator and tries to achieve profit maximization by the optimal usage of generation and load.

In normal grid-connected mode of the MMG system, the consumers can participate in energy market by providing interruptible loads [1]. In this way, consumers can shift the operation of their loads by shifting their load consumption from peak periods to off-peak periods [9]. The centralized controllers such as CAMC and MGCC help the consumers to shed the desired loads and achieve energy savings and reasonable profit margins [12]. These centralized controllers should not have their primary motivation as profit maximization in market 
participation but their primary goal should be to serve load and heat demand and to achieve improved power quality and better efficiency in the MMG system, as depicted in Figure 2-3 [1]. Different research works on centralized control of microgrid systems are available in literature [15]-[19].

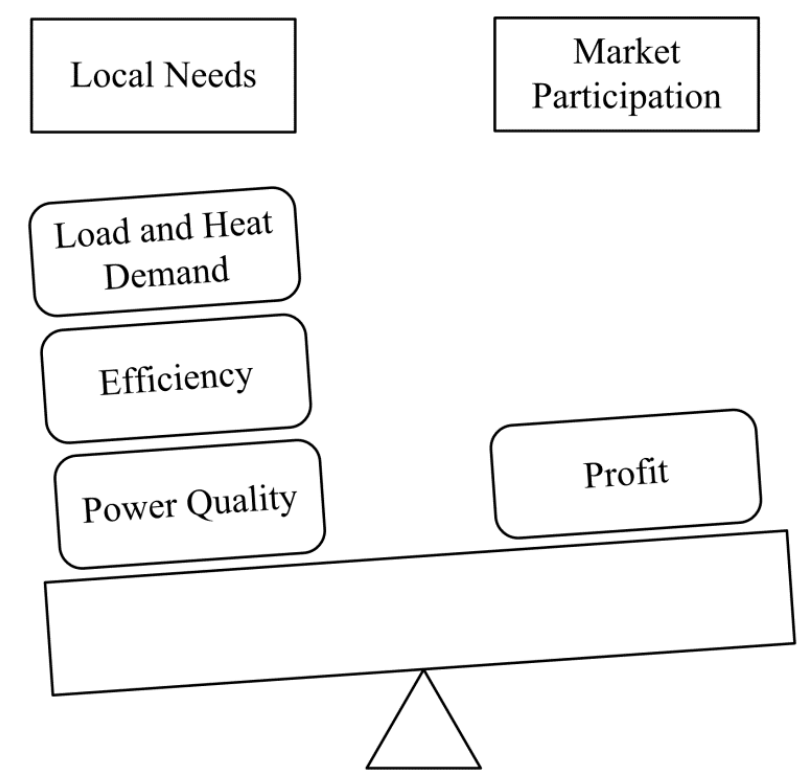

Figure 2-3: Centralized controller must give more weightage to local needs than market participation [1].

\subsubsection{Decentralized Control Scheme}

Decentralized control scheme is also being used nowadays in microgrid and MMG systems. In this scheme, the DGs, inverters and loads are controlled individually by local controllers and these controllers interact and communicate among each other to achieve optimal operation of the MMG system [9]. Operation and control of decentralized systems is based on multi-agent system (MAS) theory [9]. Many studies on decentralized control of microgrid systems are conducted in [20]-[24] and also MAS theory is explained and implemented in several microgrid systems in [25]-[29].

\subsubsection{MAS Theory}

From literature, the basic features of MAS theory are given as follows [1]:

- An agent is defined as a physical or virtual device that directly controls a DG unit or a battery unit, e.g., it can be a piece of software that controls a DG unit.

- An agent can modify the environment in which it operates, e.g., a battery unit, by modifying its power output, can alter the power outputs of other adjacent DG or battery units. 
- Agents need to maintain a good level of communication capability to achieve a reliable operation of the microgrid or MMG system, e.g., consider a system that includes a DG unit and a battery unit. The battery unit charges itself by taking energy from the DG unit and it discharges when the power output of DG unit is less. To achieve this coordinated operation, the two agents of battery and DG units should communicate effectively.

- Agents can divide the level of autonomy of their operation, e.g., a battery unit can decide when to get charged or discharged. It can decide to get charged when the cost of power supplied by grid is low and it can also decide to discharge when the cost of power supplied by grid is high.

- Agent needs to have knowledge of its local environment but is not necessarily required to know the details of the entire system which is one of the main ideas of MAS theory.

- An agent has special skills or services, which are unique, e.g., an agent whose primary aim is to provide uninterruptible power supply to a load has a different behaviour from an agent whose goal is to mainly achieve profits in the energy market.

\subsection{Advantages and Disadvantages of Centralized and Decentralized Control Schemes}

The centralized scheme and not decentralized scheme is adopted in this thesis for the operation and control of the MMG systems due to following advantages of centralized scheme over decentralized scheme.

\subsubsection{Advantages and Disadvantages of Centralized Control Scheme}

Centralized control scheme is suitable for a MMG system which has a common goal or operational aim, e.g., centralized control scheme is suitable for an industrial MMG system in which the complete ownership of the MMG system lies in the hand of a single owner [9]. Dedicated personnel can also be employed to manage the centralized operation of the whole MMG system [9]. In addition, optimization problem for a MMG system in centralized control scheme will have limited objectives such as minimization of fuel cost and limited constraints such as minimum and maximum operating costs [10]. Also, centralized controller is more preferable to enable the interconnection of several MMG systems and for setting up a largescale energy market for energy trading among MMG systems and the distribution grid in future.

If centralized controller fails, the local controllers will take over the function of controlling the individual microgrids although the power exchange between the microgrids cannot be 
maintained at the desired value. Even though high communication needs are required for a centralized control scheme, a reliable and high speed communication between centralized and local controllers will ensure the effective operation of the MMG system [9]. In addition, the centralized control scheme is more suitable for a large interconnected power system like the MMG system, where high level of coordination is required among centralized and different local controllers. Moreover, standardized procedures and easy implementations are among the other advantages of centralized control scheme [30]. The main disadvantages of centralized control scheme are the increased requirements for communication and the risk of single point failure [9].

\subsubsection{Advantages and Disadvantages of Decentralized Control Scheme}

Decentralized control scheme for microgrid control provides a number of distinct advantages in several practical cases, for example, when distributed generation (DG) units have different owners and different objectives [1]. For example, DG owners might have different objectives such as heat production for DG units, providing backup system for local critical loads, maintaining voltage locally at a certain level, and so on, and these diverse objectives cannot be easily scheduled centrally [1]. Moreover, DG owners in a microgrid operating in a market environment would most probably prefer that several decisions are taken locally by their intelligent controllers, rather than accepting set-points dictated by a centralized controller [1]. Such characteristics favour the application of decentralized control.

Distributed control scheme, a non-centralized control scheme similar to decentralized control scheme, has several advantages such as it does not require a dedicated communication network and a centralized controller to coordinate the DG units in a microgrid [1]. In this control method, the DG units have autonomous local controllers and there is an effective communication among these local controllers over a sparse communication network [1]. In addition, this method allows for the plug and play of new DG units and loads. Thus the distributed control provides better flexibility and extensibility over the centralized control method [1].

In a decentralized control scheme, the main disadvantage is that there is less coordination between centralized and local controller [1]. Thus, the connection of the whole MMG system directly as single entity to the distribution grid will be difficult [9]. This results in reduced participation of DG and storage battery units of the MMG system in the wholesale energy market [1]. For this scheme, high capital investment is required to install local controllers for 
all the individual microgrids of the MMG system and to satisfy the high communication requirements between these microgrids [9]. In order to manage high-voltage and mediumvoltage distribution grids, high levels of peer-to-peer communication are needed which might be higher than the centralized communication requirements [1]. Also, in case of decentralized scheme, multiple owners, one for each microgrid system in the MMG system will create uncertainty over what each owner wants at any particular moment [9].

\subsection{Control Schemes Adopted by Local Controllers in the MMG System}

Local controllers are used to control the DG and storage battery units in the MMG system [1]. With appropriate instructions from the centralized controller, the local controllers help in efficient power sharing among DG and storage battery units, and effective voltage and frequency regulation in the MMG system [9]. Different DG units such as PV, wind, and microturbine are connected to the load bus of the MMG system through several converters such as AC/DC, DC/AC and DC/DC converters. Several control schemes are available to control the output currents of these converters during grid-connected and islanded modes of the MMG system [9]. These control schemes are generally classified into classical and nonclassical control schemes which are explained as follows:

\subsubsection{Classical Control Schemes}

Over the last few decades, current control has been used extensively for power converters. Among the classical control schemes, the most popular are hysteresis control and linear control using pulse width modulation (PWM) [31]-[36].

\subsubsection{Hysteresis Current Control}

Basic principle of hysteresis current control is to keep current error within a desired upper and lower limit or band [31]. In Figure 2-4(a), hysteresis control scheme for a single-phase inverter is shown. Here if the current error is greater than the upper limit $\delta / 2$, then the switches $\mathrm{T}_{1}$ and $\mathrm{T}_{4}$ are turned on [36]. If the current error is less than the lower limit $-\delta / 2$, then the switches $T_{2}$ and $T_{3}$ are turned on [36]. From Figure 2-4(b), it can be seen that the load current $i_{L}$ follows its reference $i_{L}{ }^{*}$ very closely. This method is simple to implement and has a good dynamic response [36]. The disadvantage of this method is that switching frequency varies with the variation in the hysteresis band and a variable switching frequency causes resonance problems and switching losses [31]. These disadvantages restrict hysteresis 
control to lower power levels [31]. Also, a very high sampling frequency is needed to implement hysteresis controller in a digital control platform [36]. Due to these disadvantages, hysteresis control is not suitable for inverter control in the proposed MMG systems in this thesis.

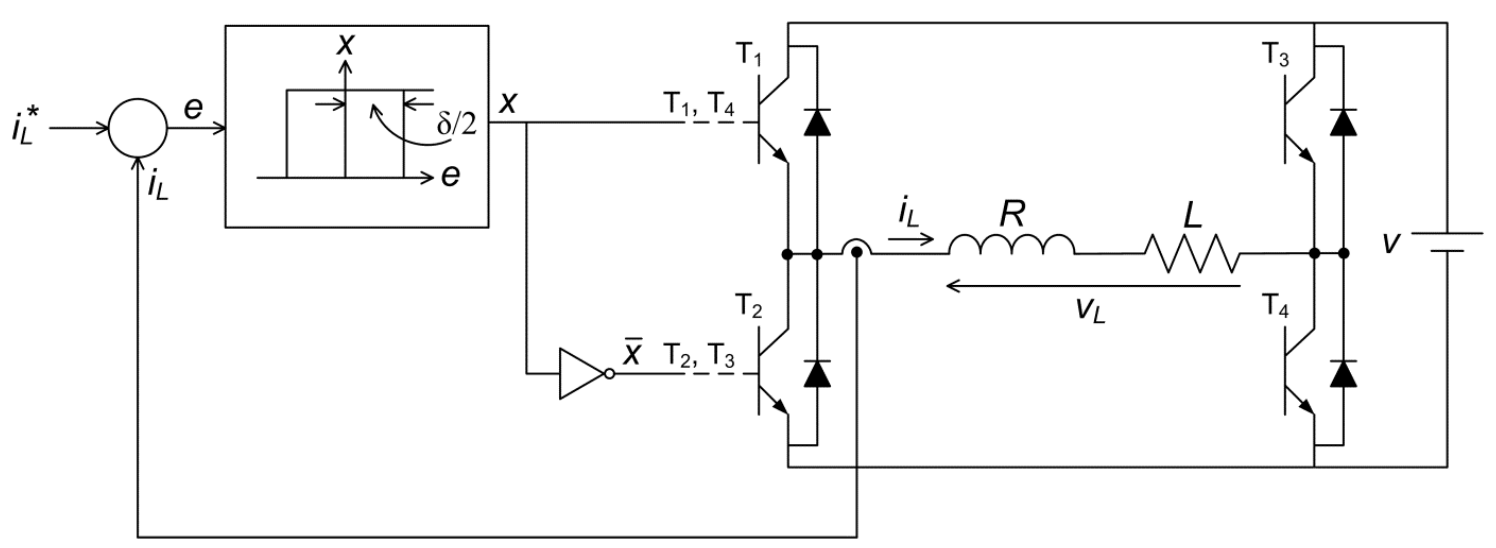

(a)

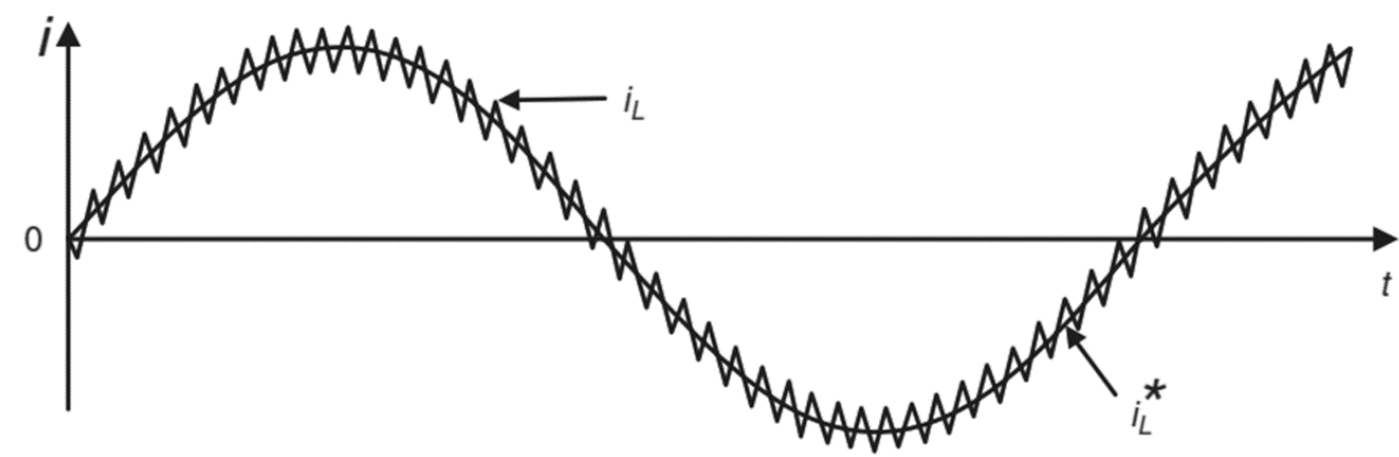

(b)

Figure 2-4: Hysteresis current control for a single-phase inverter: (a) Control scheme, (b) Load current [36].

\subsubsection{Linear Control with Pulse Width Modulation (PWM)}

PWM of a single-phase inverter is shown in Figure 2-5 (a). In this method, a triangular carrier wave signal is compared with reference voltage and the voltage error signal is then fed into a comparator [32]. The output of this comparator is then then used to drive the inverter switches [33]. Generally, a proportional-integral (PI) controller is used as a comparator [33].

Figure 2-5(b) shows the waveforms for PWM. The amplitude of the carrier wave is same as that of the reference voltage [36]. A pulsed voltage waveform is generated by comparing a triangular carrier wave with a reference sinusoidal voltage [36]. The reference voltage is proportional to the fundamental component of this pulsed voltage [36]. 
The performance of PI controller depends mainly on the proportional and integral gains, and the frequency of the reference current [34]. An advantage of PI controller is that it gives a zero steady-state error in case of continuous fixed reference but its main disadvantage is that it gives a noticeable error in case of sinusoidal references [35]. Thus, in case of sinusoidal references, the different variables involved are converted from the original coordinate transformation into a rotating reference frame, where the reference currents are constant values [36]. This will add on to the complexity of the controller [36]. These disadvantages make the linear control unsuitable for the control of multiple inverters in the proposed MMG systems in this thesis.

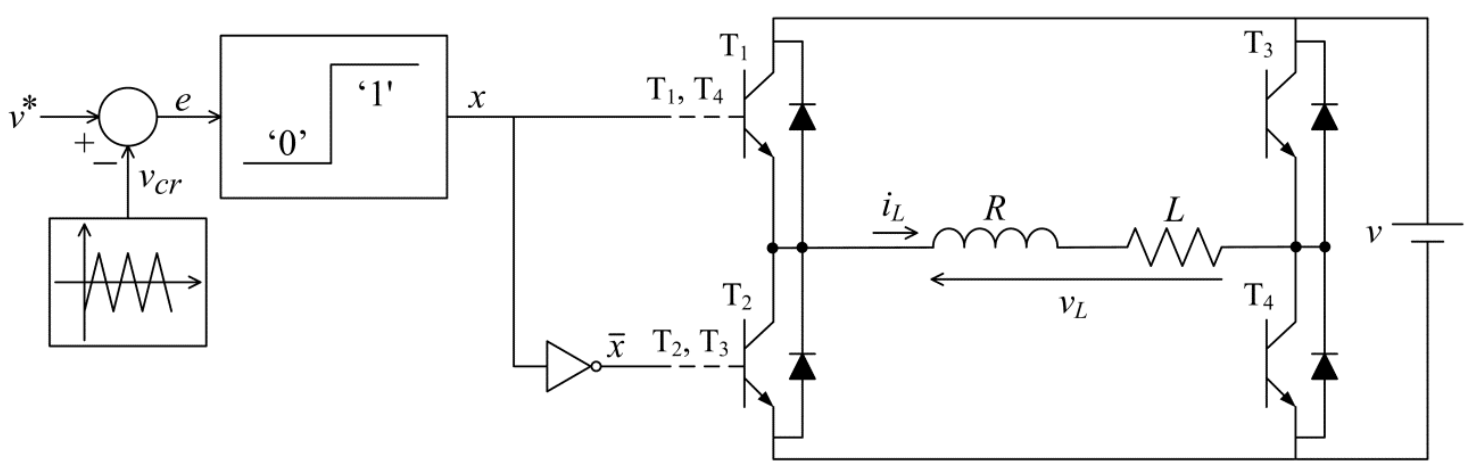

(a)

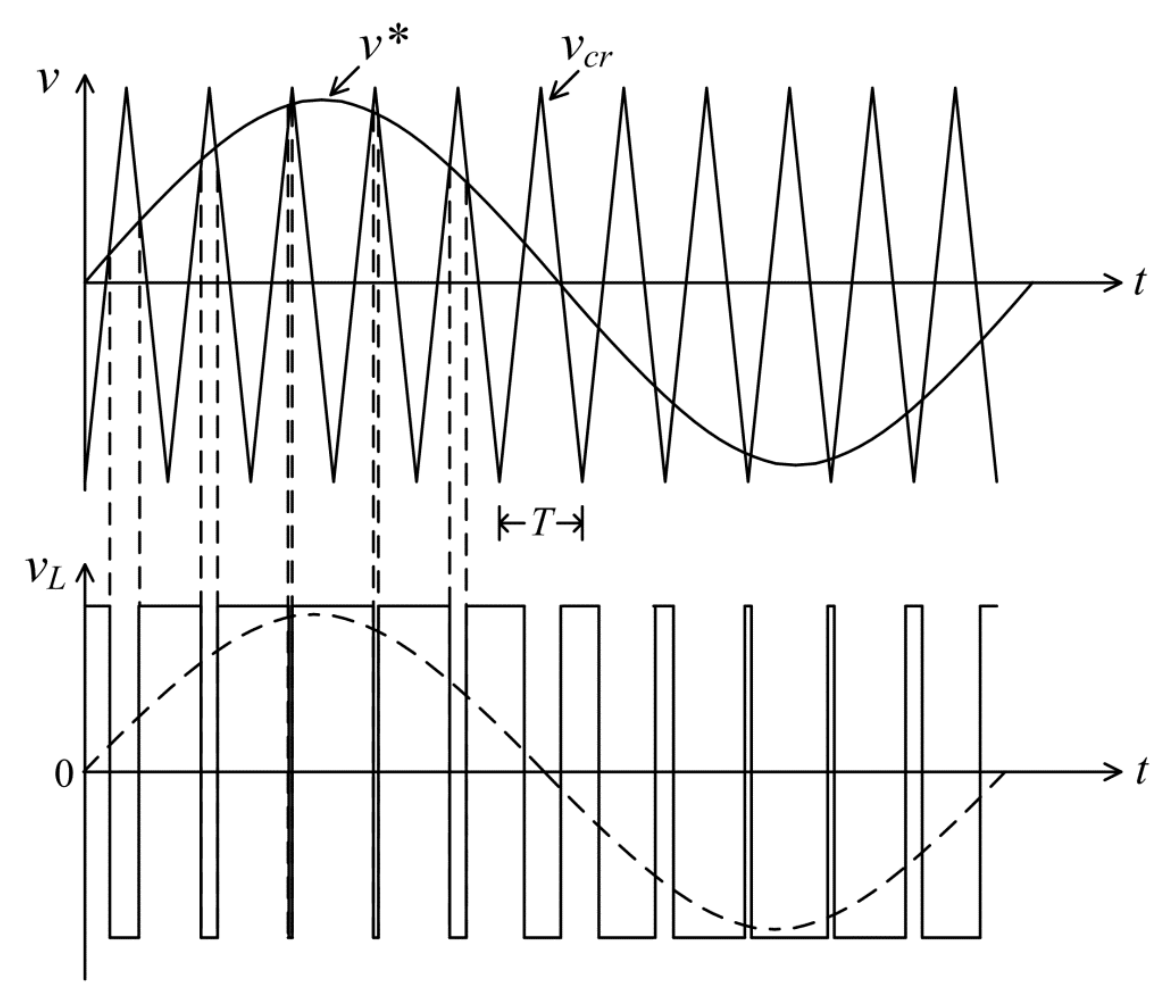

(b)

Figure 2-5: Pulse width modulation for a single-phase inverter: (a) Control scheme, (b) Waveforms [36]. 


\subsubsection{Non-classical Control Schemes}

With the advent of powerful fast microprocessors and workstations, new non-classical control schemes such as sliding mode, fuzzy logic and predictive control have come into existence [36]. In sliding mode control, the switching nature of the power converters is considered and in fuzzy logic control, the parameters of the controlled system are not known [36]. Predictive control is an interesting control algorithm that has become popular for converter control in recent years [37]. Various advantages of predictive control are that it is easy to understand its concepts; it can easily include constraints and non-linearities and it can consider different multi-variable cases [36]. One disadvantage of predictive control is that it involves a lot of mathematical calculations [37]. But using the fast powerful microprocessors available currently, these calculations can be easily performed [37]. Based on the advantages mentioned, predictive control is the preferred control method used for various power converters in the proposed MMG systems in this thesis.

\subsubsection{Predictive Control Methods for Power Converters}

There are different classifications of predictive control which are shown in Figure 2-6. The main principle of predictive control is the use of a system model to predict the future characteristics or behaviour of the variables to be controlled [36]. Based on the pre-defined optimization criterion and system model, the predictive controller is required to obtain the optimal actuation [36]. The optimization criterion is different in different types of predictive control [37]. In hysteresis based predictive control, the criterion is used to limit the controlled variables within a desired hysteresis area [38]. In trajectory based predictive control, the controlled variables are forced to follow a fixed trajectory [36]. In deadbeat based predictive control, the optimal switching state is the one which makes the error between the reference and the measured variable equal to zero in the next sampling instant [39], [40].

In MPC, the optimization of a pre-determined cost function is performed taking into consideration different constraints [36]. The difference between these predictive controllers is that some of them require a modulator while others do not require a modulator [36]. Deadbeat control and MPC with continuous control set require a modulator while other predictive controllers do not need a modulator [37]. Also, deadbeat control and MPC with continuous control set have a fixed switching frequency while other predictive controllers have variable 
switching frequency [37]. MPC with finite control set does not require a modulator and is relatively simple to implement [36]. One disadvantage of MPC with finite control set is that it is complex for a multi-level inverter with complex topologies [36].

A significant advantage of MPC over linear PI control is that it is not necessary to use cascaded outer and inner control loops, and coordinate transformations [41]. Non-linearities of the system model can be easily included in MPC, which results in elimination of the procedure of linearization required for non-linear control schemes [36]. Thus, taking into consideration all the advantages of MPC and disadvantages of different linear control schemes, the control of converters in the proposed MMG systems in this thesis is implemented using MPC.

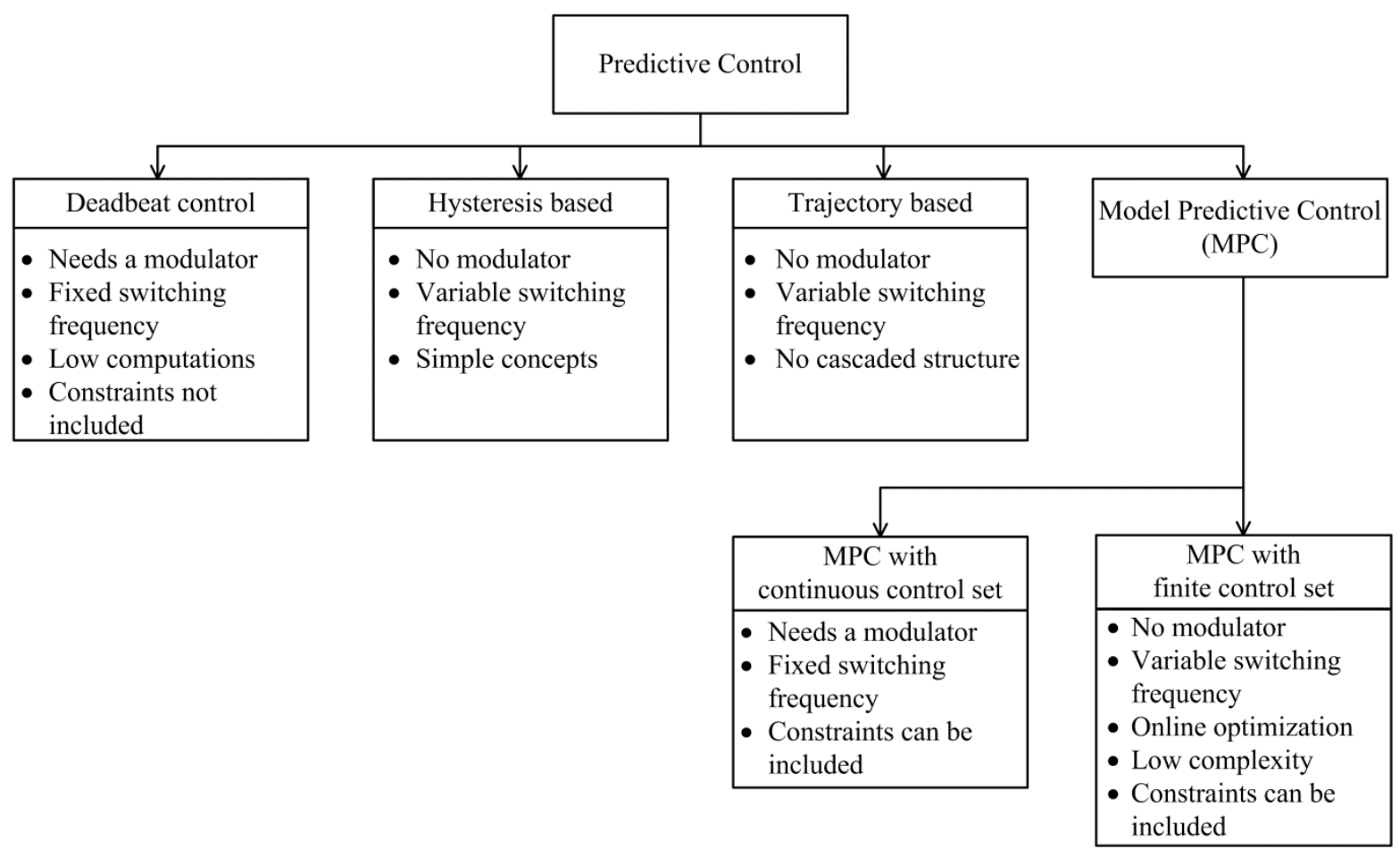

Figure 2-6: Classification of predictive control methods used in power electronics [36].

\subsubsection{Model Predictive Control (MPC)}

MPC has been used in chemical process and power electronic applications since 1980 onwards [36]. But higher switching frequencies for power electronic switches were not possible at that time due to higher number of calculations required [36]. So MPC was not popular in 1980s [36]. However, with the advancement in the development of fast 
microprocessors, MPC has become increasingly popular in recent years based on several advantages as follows [36]:

- It can be applied to single-input single-output and multi-input multi-output systems.

- Non-linearities and constraints can be easily incorporated.

- Easy implementation.

The basic principles of MPC are given as follows [42]:

- Use of a system model to predict the future behaviour of controlled variables.

- Definition of a suitable desired cost function.

- Minimization of the cost function to obtain the optimal switching state or actuation of the converter.

The discrete state-space model used for MPC [36] is given as

$$
\begin{gathered}
x(k+1)=A x(k)+B u(k) \\
y(k)=C x(k)+D u(k)
\end{gathered}
$$

where $k$ is the sampling instant, $x(k), u(k)$, and $y(k)$ are the state, input and output variables respectively, and $A, B, C$ and $D$ are the state, input, output and feed-forward matrices respectively.

Cost function depends on the reference variables of the system [42]. It is a function of state variables, future states, actuations, future actuations and references as follows [36]:

$$
J=f(x(k), u(k), \cdots, u(k+N))
$$

MPC with finite control set involves a problem of optimization, whose objective is to minimize a cost function $J$ for a pre-defined horizon in time ' $N$ ' subject to system model and constraints [43]. The overall result of optimization is the finite sequence of $N$ optimal actuations [42]. The MPC controller then applies only the first element of the finite sequence given below [42].

$$
u(k)=\left[\begin{array}{llll}
1 & 0 & \cdots & 0
\end{array}\right] \arg \min _{u} J
$$

The working principle of MPC is illustrated in Figure 2-7. Using the system model and available measurements, the future values of system states are predicted by MPC until time $k+N$ [36]. By minimizing the cost function, the sequence of optimal actuations is obtained and first element of this sequence is applied [36]. This process of prediction of system states and minimization of cost function is repeated again and again for each sampling instant [42]. 
MPC theory was developed earlier in 1970s itself. But due to faster sampling times required, MPC could not be implemented [36]. However, in recent times, faster microprocessors made the implementation of MPC possible [36]. There are mainly two different types of MPC as shown in Figure 2-6. In MPC with continuous control set, non-linear model of the converter has to be linearized and an additional modulator is required [42]. But in MPC with finite control set, non-linearities and constraints of the converter model can be easily included [43], [44]. Considering the discrete nature of power converters and finite switching states of these power converters, the optimization problem is solved online itself in case of a finite control set MPC [42]. These factors make MPC with finite control set more flexible and simple than MPC with continuous control set and thus MPC with finite control set is the preferred MPC control technique for various power converters used in the proposed MMG systems in this thesis.

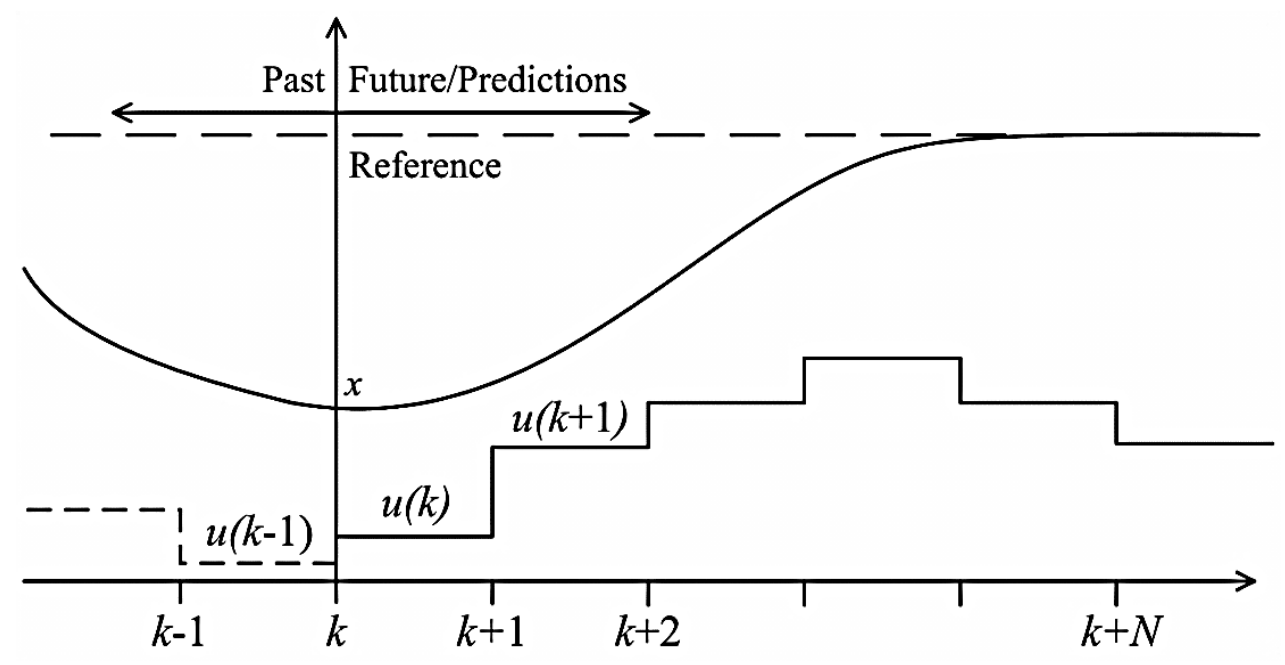

Figure 2-7: Working principle of MPC [36].

There are basically two stages for developing finite control set MPC for power converters [36]. The first stage is the design stage and the second stage is the implementation stage [36].

Design stage of finite control set MPC consists of the following steps [42]:

- An appropriate converter model must be developed considering all possible switching states.

- A cost function must be defined for representing system behaviour.

- A discrete-time model allowing the prediction of future behaviour of the controlled variables is needed.

The number of possible switching states $N$ for an inverter is [36] 


$$
N=x^{y}
$$

where $x=$ number of possible states of each converter leg of the converter

$y=$ number of phases of the converter

For example, in case of a three-phase three-level inverter, the number of possible switching states is $N=3^{3}=27$.

Depending upon the application of MPC, the control requirements such as torque control, current control and power control are imposed [36]. In general, a simple cost function is defined as the difference between the predicted and reference variable [36]. Predicted variable can be load current, power, torque, etc. [36]. The error calculated with cost function can then be load current error, power error, torque error, etc. [36]. In some cases all the output variables may not be measured and some of them may be estimated [42], [44].

Using Euler forward method, the continuous state-space model can be converted into discrete state-space model as follows [36]:

$$
\frac{d x}{d t}=\frac{x(k+1)-x(k)}{T_{s}}
$$

where $T_{s}$ is the sampling time. For higher order systems, Euler method fails because the error introduced by this method is very high.

In the implementation stage of MPC, the following tasks should be considered [42]:

- Prediction of future behaviour of the controlled variables

- Evaluation of cost function for each prediction

- Selection of optimal switching state that minimizes the cost function

Different processors such as fixed-point and floating point processors can be used for MPC implementation [36]. Sampling time and switching states are important variables which decide the time taken for performing the calculations in MPC method [36]. In case of threephase three-level inverter, prediction of the controlled variable for eight possible switching states is not a problem but for multi-phase multi-level inverter, a different optimization method must be considered to reduce the number of calculations [36].

A typical MPC control scheme for power converter is shown in Figure 2-8. Here the measured variable $x(k)$ is used to calculate the predicted variable $x(k+1)$ for ' $n$ ' possible 
switching states [42]. Then a cost function is evaluated which decides the error between predicted variable $x(k+1)$ and reference variable $x^{*}(k)$ at each sampling instant [42]. The optimal switching state $S$, which gives minimum cost function, is selected and applied to the converter [42].

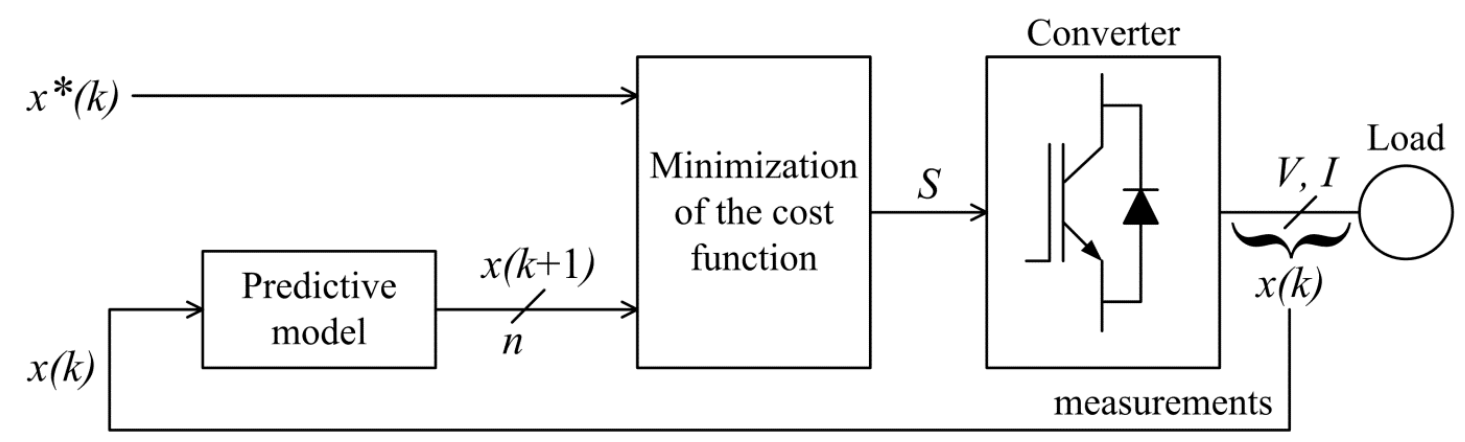

Figure 2-8: Typical MPC scheme for power converter [42].

Different conventional voltage and power control methods using droop control are available to control the inverters in microgrid systems [45]-[48]. However, power sharing performance with conventional droop control degrades with variations in transmission line impedances in interconnected inverter-based microgrid systems [47], [48]. To overcome these drawbacks of droop control, MPC has been increasingly used in recent years for the control of converters [36], [42]. In [36], [42], a finite control set MPC which allows for the control of different converters without the need of additional modulation techniques is presented. In [49], [50], an investigation on the usefulness of MPC in the control of parallel-connected inverters is conducted. However, further research is needed for the application of MPC in the control of converters in MMG systems.

Different from these existing MPC methods, the state-space model based MPC algorithm proposed in this thesis is independent of the system parameters such as grid, line and load impedances of the proposed MMG systems. Thus, the proposed MPC guarantees a fast dynamic response and a small steady-state error under different operating modes of the microgrids such as grid-connected and islanded modes. The details about these operating modes will be explained in the next section. Also, MPC gives a better transient response as compared to other conventional control methods [36]. The remarkable features that make MPC an ideal technique for the control of converters is shown in Figure 2-9. In view of the aforementioned advantages of MPC, the power control scheme for the converters is implemented in the proposed MMG systems using MPC. 


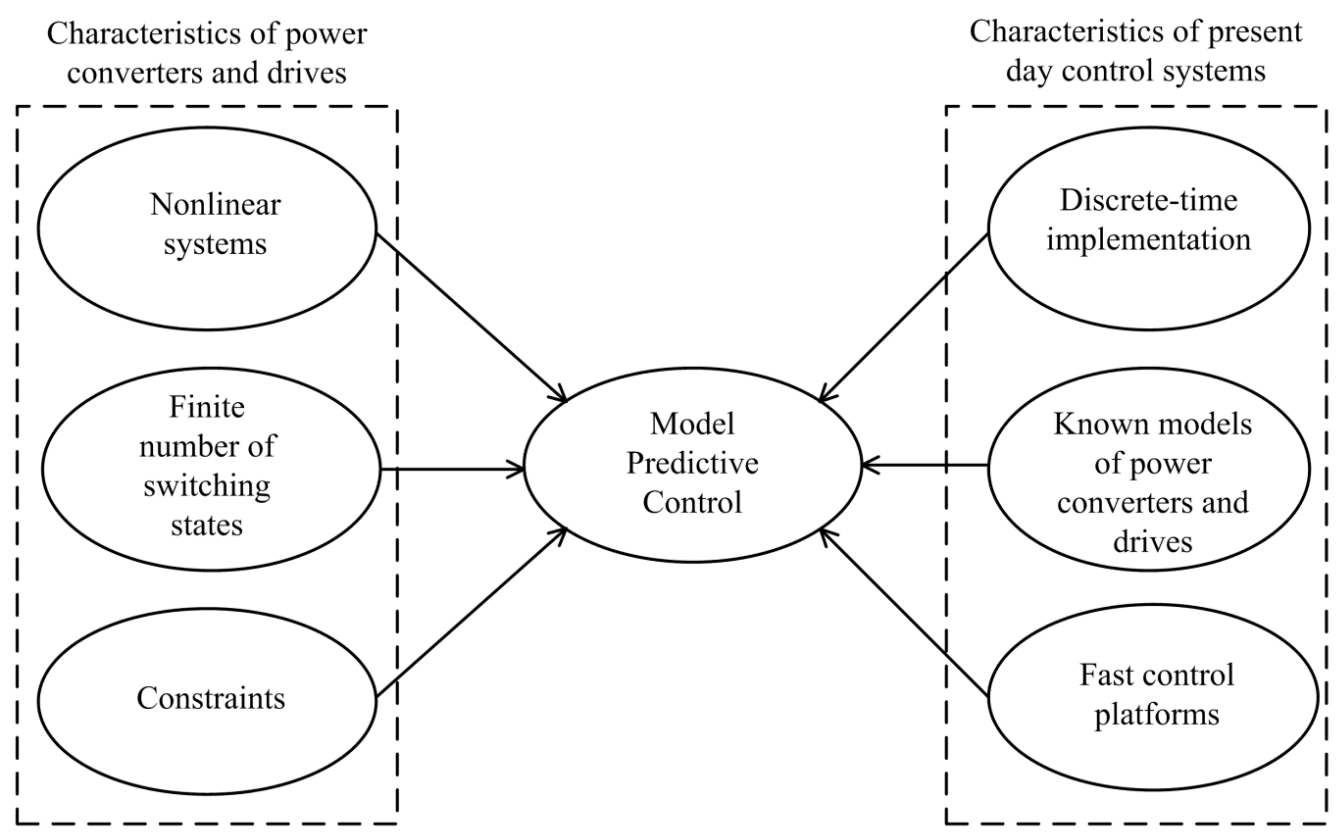

Figure 2-9: Features of MPC that make it an ideal control technique for converters [36].

The novel contributions of the proposed MPC algorithm in Chapter 3 of this thesis, which distinguish itself from the existing MPC algorithms are as follows:

a) The control performance of the proposed MPC algorithm for the inverter depends only on the inverter parameters such as inverter loss resistance and inverter's filter inductance and capacitance, as inferred from the state-space model of the inverter given in (3.11). However, the performance of the existing MPC algorithm given in [36] depends on load impedances of the microgrid system, as inferred from the state-space model of the existing MPC algorithm. As a result, the performance of the proposed MPC algorithm is independent of load impedances of the microgrid system whereas the performance of the existing MPC algorithm given in [36] depends on load impedances of the microgrid system. The proposed MPC algorithm can thus be used for multiple inverters and different load impedances without modification of the state-space model of the inverters. Therefore, the main advantage of the proposed MPC algorithm is its scalability and implementation for multiple inverter modules in a large multi-microgrid system.

b) In the proposed MPC algorithm, two steps of reference are used in the cost function given in (3.13). But in the existing MPC algorithm given in [36], only one step of reference is used in the cost function. Thus, a proper choice of the control signal is obtained resulting in an improved control performance in the proposed MPC algorithm in comparison with the existing MPC algorithm given in [36]. 
c) The proposed MPC algorithm reduces the computational burden of another existing MPC algorithm which is performed by using quadratic programming as given in [109]. Thus, the proposed MPC algorithm guarantees a fast dynamic response during different operating modes of the interconnected microgrids in the multi-area multi-microgrid systems developed in this thesis.

\subsection{Operation Modes of Microgrids in the MMG System}

A microgrid in the MMG system generally operates in two modes [51]:

- Grid-connected, when the microgrid is connected to the distribution grid and it operates in close coordination with the distribution grid.

- Islanded mode, when the microgrid is disconnected from the distribution grid and it operates autonomously with self-regulation of voltage and frequency.

In Figure 2-10, power exchange between microgrid and grid is shown. The microgrid consists of DG, energy storage (ES) and load [51]. Grid is represented by the voltage source E. P is the real power exchange between grid and microgrid, and $Q$ is the reactive power exchange between grid and microgrid [51]. Grid-connected mode of a microgrid is sub-divided into two operation modes as follows [51]:

- Power matched operation when $\mathrm{P}+\mathrm{Q}=0$, i.e., when generation and load reach a balance and no power exchange occurs.

- Power mismatched operation when $\mathrm{P}+\mathrm{Q} \neq 0$. When $\mathrm{P}+\mathrm{Q}>0$, real and reactive power flows from grid to microgrid to satisfy the load demand in the microgrid. When $\mathrm{P}+\mathrm{Q}<$ 0 , then excess real and reactive power flows from microgrid to grid.

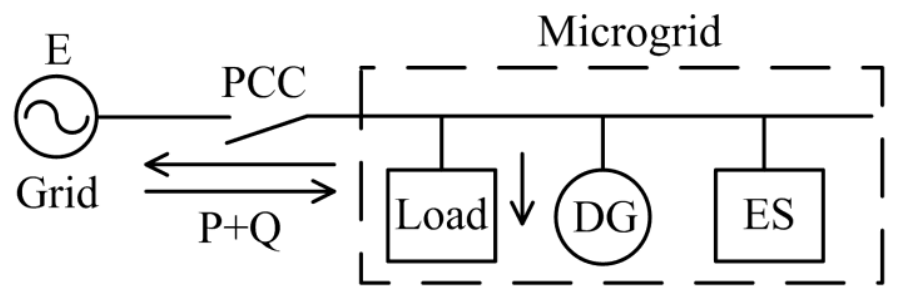

Figure 2-10: Power transfer from grid to microgrid and vice-versa [51].

\subsubsection{Grid-Connected Mode}

In grid-connected mode, the microgid is connected to the distribution grid and exchanges power with the distribution grid via point of common coupling (PCC) [51]. In grid-connected mode of the microgid, the grid regulates the voltage and frequency of the microgrid [51]. All the inverters connected to the DG and storage battery units in the grid-connected microgid are 
operated in current control mode (CCM) to share the load demand [51], [52]. In CCM, the output real and reactive powers of the inverters in the MMG system are regulated at the desired reference values [51]. Thus, in grid-connected mode, the main responsibility of the microgrid is to ensure accurate power sharing among the distribution grid and the DG units [52]-[55]. Figure 2-11 shows the schematic diagram of transfer between different operation modes of the microgrid. The transfer of microgid between different operation modes is explained as follows [51]:

- A grid-connected microgrid can be disconnected from the grid by disconnection control.

- A grid-connected microgrid can shift to shut down condition by shut down control. In the shut down condition, the microgrid is disconnected from the grid and all the DG units, battery units and converters/inverters are disconnected from the load bus. A microgrid is shut down for the purpose of scheduled maintenance and servicing of its components such as DG units, battery units and converters/inverters [51].

- An islanded microgrid can shift to grid-connected mode by grid connection control and can shift to shut down condition by shut down control.

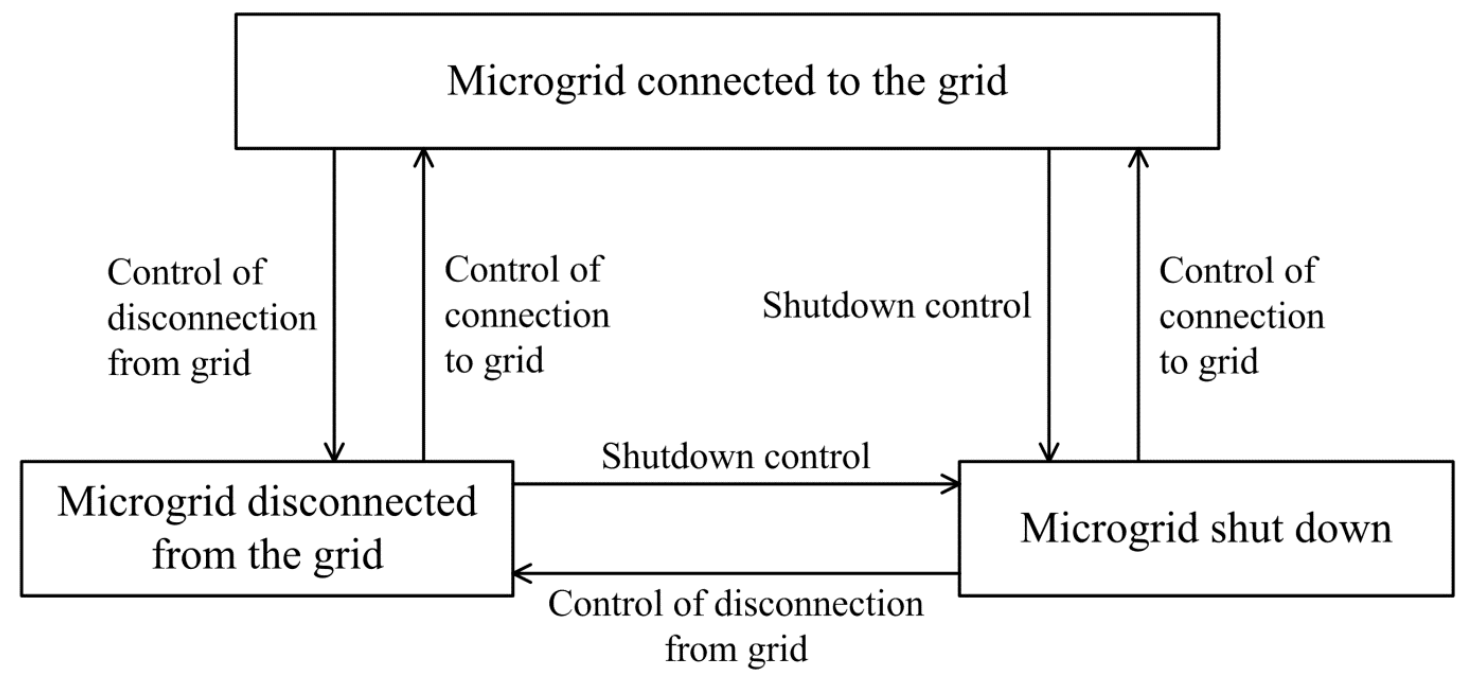

Figure 2-11: Transfer between operation modes [51].

\subsubsection{Islanded Mode}

In islanded mode, the microgrid is disconnected from the grid due to failure or disturbance in grid power supply [51]. Under this situation, microgrid needs to operate independently. DG and storage battery units in the islanded microgrid share the additional power demand, which was supplied by the grid before disconnection [51]. The DG or storage battery unit in the islanded microgid regulate the load bus voltage [51]. Usually the battery unit is made to 
regulate the load bus voltage since the battery unit is a more stable and reliable power source than the other intermittent DG units [51]. The islanded microgrid must be reconnected to the grid once the disturbance is removed [53]. In case the DG and storage battery units are unable to meet the load demand in the islanded microgrid, underfrequency load shedding is implemented in the islanded microgrid, if the islanded microgrid is an AC microgrid and undervoltage load shedding is implemented in the islanded microgrid, if the islanded microgrid is a DC microgrid. Since microgrid islanding is a very important operation, many studies [56]-[63] are focussed on this operation to achieve a seamless transition from gridconnected to islanded mode and also to attain a stable and reliable operation of the islanded microgrid. In this thesis, grid-connected, islanded and load shedding operations are carried out in the interconnected microgrids in the proposed MMG systems to test the performance of these systems under various operating conditions.

\subsection{Control Modes of Microgrids in the MMG System}

The commonly used control modes of microgrids in the MMG system are master-slave mode, peer-to-peer mode and combined mode [51].

\subsubsection{Master-Slave Mode}

In master-slave mode, one of the DG units in the microgrid acts as the master unit controlling the load bus voltage and the other DG units act as slave units by following the voltage reference fixed by the master unit [51]. Master-slave mode of a microgrid is shown in Figure 2-12. In grid-connected operation of a microgrid in the MMG system, all the DG units operate in CCM or real/reactive power (P/Q) control [51]. During islanding of a microgrid in the MMG system, one DG unit, which can give reliable and stable power supply, becomes the master unit and operates in voltage/frequency (V/f) control mode to regulate the load bus voltage [51]. Other DG units serve as the slave units, i.e., these DG units operate in CCM and follow the load voltage reference fixed by the master unit [51]. Different DG units with variable outputs such as PV or stable controllable DG units such as fuel cell, battery and microturbine can be used as master unit [51]. Master-slave control mode is widely used in literature [64], [65]. This control mode has several advantages as follows [51]:

- Only a simple control algorithm is required at each component level.

- Master-slave control unlike other peer-to-peer control modes does not depend on impedances of the lines interconnecting the microgrid systems in the MMG system. 
Disadvantages of master-slave control are as follows [51]:

- Once the master DG unit fails, there is a chance of collapse of the whole microgrid system but if another DG unit takes the role of the master unit, this collapse can be averted.

- A high expenditure on communication and cabling system is needed but a reliable and high speed communication and cabling system will improve the efficiency and reliability of master-slave mode.

Thus, based on the aforementioned advantages of master-slave control mode, this control mode is used to control the inverters and converters in the proposed MMG systems in this thesis. During islanding of a microgrid in the MMG system, the battery and DG inverters in the islanded microgrid act as the master and slave inverters respectively [64]. In this islanded microgrid, the storage battery inverter operating in voltage control mode (VCM) or V/f control mode regulates the load bus voltage while the DG inverter operates in CCM or P/Q control mode [65].

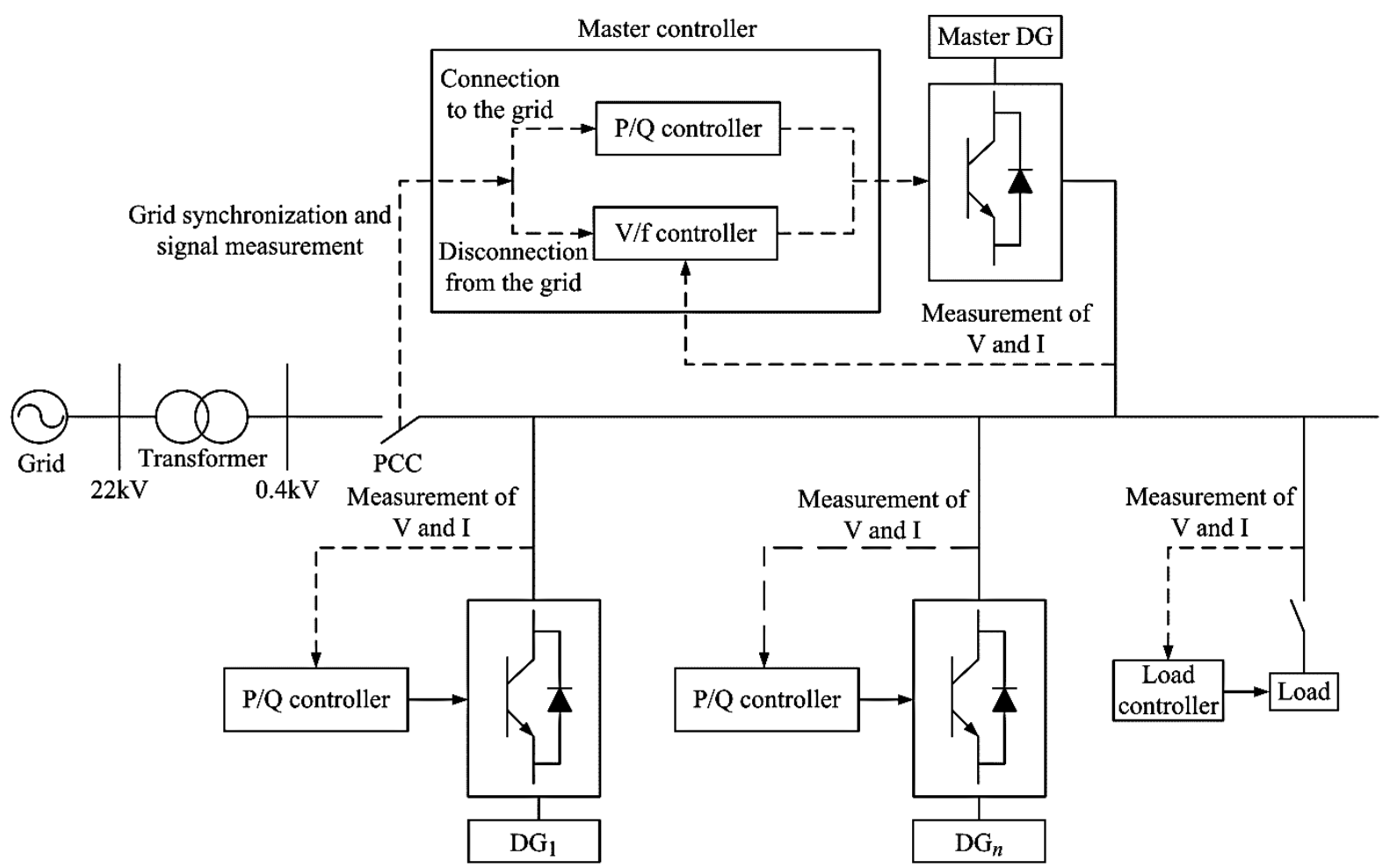

Figure 2-12: Architecture of microgrid under master-slave control mode [51]. 


\subsubsection{Peer-to-Peer Mode}

Peer-to-peer mode is based on "plug-and-play" and decentralized control scheme [51]. It depends on the interaction between the DG units in a microgrid for achieving the goal of voltage and frequency control [51], [66]. Here all DG units are equal and there are no master and slave units [51]. Droop control is a commonly used peer-to-peer control mode with the architecture shown in Figure 2-13. Different types of droop control can be found in literature [45]-[48]. In this control mode, if load changes, then that load change will be distributed among the DG units according to their droop factors [51]. This allows for the autonomous control of the DG units especially during microgrid islanding [51]. Droop control is actually a type of proportional control [51]. When the microgrid is islanded, the droop control will enable the DG units to share the real power proportionally to regulate the microgrid system frequency $f$ to its nominal value $f_{0}$ [51]. Also, droop control will facilitate proportional sharing of reactive power demand by the DG units in the islanded microgrid to regulate the load voltage $V$ to its nominal value $V_{0}[51]$.

The sharing of real and reactive powers is achieved through the droop control method [18] by regulating the magnitude and frequency of the DG unit, as described by the following equations:

$$
\begin{aligned}
& f=f_{0}-D_{p} P \\
& V=V_{0}-D_{Q} Q
\end{aligned}
$$

where $f$ and $V$ are the actual operating frequency and output voltage of the DG unit, $f_{0}$ and $V_{0}$ are the reference frequency and output voltage when the microgrid operates in islanded mode, $P$ and $Q$ are the output real and reactive powers of the DG unit, and $D_{p}$ and $D_{Q}$ are the droop parameters or coefficients of the DG unit.

The method of determining the droop parameters of the DG unit is explained as follows:

The droop parameters can be determined either heuristically or by tuning algorithms (e.g., particle swarm optimization). In the former approach the droop parameters are determined based on the DG unit power ratings and the maximum allowable voltage and frequency deviations [47]. For instance, in the microgrid shown in Figure 2-13, with $n$ DG units, the corresponding droop parameters $D_{p}$ and $D_{Q}$ should satisfy following constraints [47]:

$$
D_{p 1} P_{1}=D_{p 2} P_{2}=\cdots=D_{p n} P_{n}=\Delta f_{\max }
$$




$$
D_{Q 1} Q_{1}=D_{Q 2} Q_{2}=\cdots=D_{Q n} Q_{n}=\Delta V_{\max }
$$

where $\Delta f_{\max }$ and $\Delta V_{\max }$ are the maximum allowable frequency and voltage deviations, respectively. $P_{1}, P_{2}, \ldots, P_{n}$ are the real power outputs of the DG units and $Q_{1}, Q_{2}, \ldots, Q_{n}$ are the reactive power outputs of the DG units.

The main advantages of droop control are as follows [45], [51]:

- It does not require communication between centralized and local controllers.

- The control action is based only on local measurements of voltage and frequency in the microgrid.

Droop control will bring about new steady-state voltage and frequency values in the islanded microgrid and these new voltage and frequency values may differ from their nominal values in grid-connected mode [51]. The disadvantages of droop control are as follows [45], [51]:

- The performance of droop control degrades with variations in system parameters and line impedances in microgrid systems.

- It cannot be used with non-linear loads because it is unable to compensate the harmonic currents.

Thus, due to these disadvantages, droop control is not used in this thesis for the control of converters and inverters in the interconnected microgrids in the proposed MMG systems.

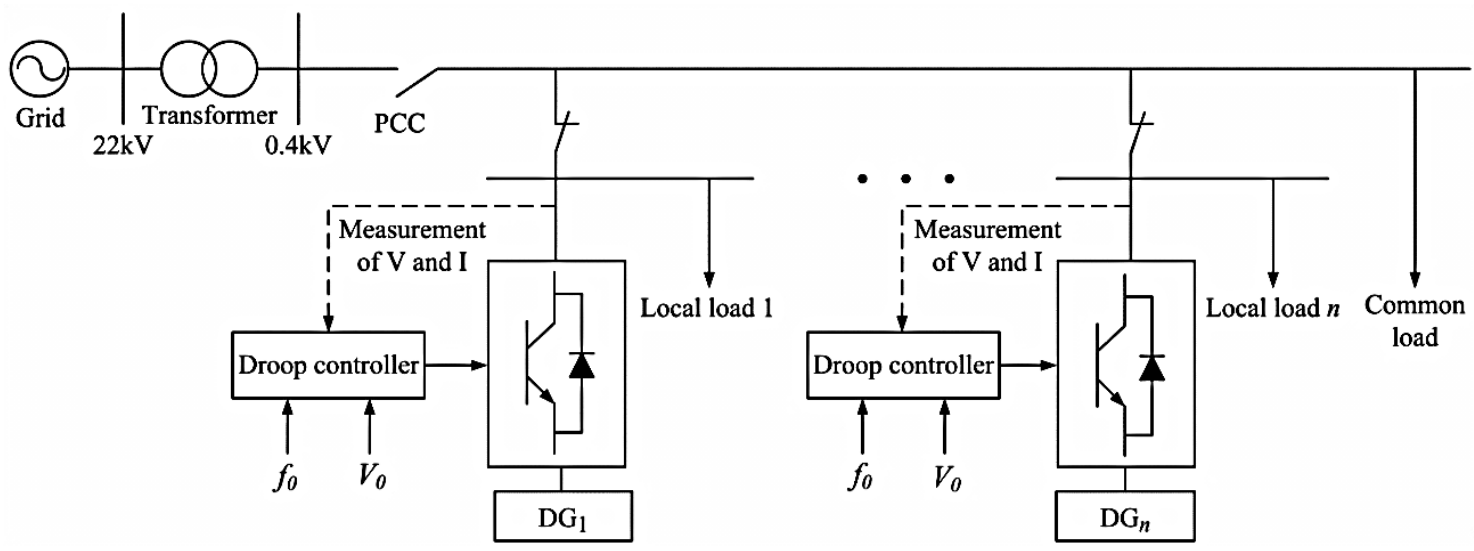

Figure 2-13: Architecture of microgrid under peer-to-peer control mode [51].

\subsubsection{Combined Mode}

Combined mode is relatively new a mode of operation in which both master-slave and peerto-peer controls can be implemented for different DG units with different characteristics [51]. In view of different types of DG units such as PV, fuel cell, microturbine, etc., different control modes such as master-slave and peer-to-peer controls can be simultaneously adopted 
in grid-connected and islanded microgrids [51]. Thus, in combined mode, the advantages of both master-slave and peer-to-peer can be utilized considering the different types and characteristic features of the DG units.

\subsection{Different Types of MMG Systems - Conventional and Proposed MMG Systems}

A literature review is done on conventional MMG systems and brief details about these MMG systems are provided here. In [1], the MMG system consists of low-voltage microgrids connected on adjacent medium voltage feeders. In [65], a multi-microgrid laboratory system consisting of two microgrids connected through an interconnection switch is presented. When this interconnection switch is opened, the two microgrids operate separately and when the switch is closed, the two microgrids combine into one large microgrid. A MMG test bed, consisting of three closely spaced microgrids connected to a common central AC bus, is developed [67], where the microgrids operate in both independent and interconnected modes. Different laboratory based small-scale multi-microgrid systems such as interlinked AC microgrids [68], DC microgrid clusters [69] and hybrid AC/DC microgrids [70]-[73] are proposed. However, in these works the grid-connected and islanded operations of the multimicrogrid systems are not explained in detail and the focus is on the control of interlinking converters between these microgrids. Also, in these research works, the MMG consists of closely spaced microgrids forming a single-area MMG system with loads in the range of kWs and where the impedances of the interconnecting lines between these microgrids are very small. In such a single-area MMG system, all the converters respond together in unison to the changes in load and in which frequency is assumed to be constant. Thus, interconnected microgrids in close vicinity forming a single-area MMG system have already been studied. But interconnected microgrids separated by large distances have not received sufficient research attention. So, if these microgrids consist of loads in the range of several MWs and the interconnecting lines have large variable impedances as in a multi-area MMG system, then the control of power flows between these microgrids using conventional droop control methods will become inefficient. Because the power sharing performance in conventional droop control depends whether the converter output impedance and line impedance is resistive or inductive [45], [51]. Thus it is difficult to operate a MMG system consisting of multiple microgrids with each microgrid having converters of variable output impedances and interconnecting lines of variable impedances. As a result, an efficient control method for the exchange of desired power between distantly spaced microgrids is essential, which necessitates the need for further research in the area of multi-area MMG. Thus, multi-area 
MMG systems formed by the interconnection of microgrids separated by large distances are proposed in this thesis and the control of these MMG systems are discussed in detail. Also, there has been no previous research on the control of multi-area MMG systems.

A multi-area MMG system is defined as an interconnected microgrid system where each microgrid is controlled independently as a separate control area and power flows in the lines interconnecting the microgrids are also controlled independently of the control areas. Different types of MMG systems are proposed in this thesis. In Chapter 3, an AC/AC multiarea MMG system consisting of interconnected AC microgrids is proposed and in Chapter 4, AC/DC multi-area MMG system consisting of interconnected AC and DC microgrids is developed. In comparison with conventional MMG systems, the main advantage of the proposed multi-area MMG system is that power flows inside an individual microgrid and power flows through the adjacent interconnecting lines of the given microgrid can be independently controlled in this system.

As size and capacity of microgrids are getting increased every year, interconnection between microgrids become essential to improve the reliability and security of microgrid systems. In this context, there exists an essential need for the deployment of multi-area MMG systems in which each microgrid is controlled by separate local controllers and the overall control rests with the centralized controller. Interconnected renewable energy systems of very high capacity, similar to the proposed multi-area MMG system, exist as in [74], [75] and are also being newly constructed in different parts of the world. Thus, the study of operation, control and power quality problems in these multi-area MMG systems is an interesting research area.

\subsection{Power Quality Problems}

A disturbance which causes the deviation of the system voltage or current from an ideal reference value is called as a power quality (PQ) problem [76]. Several PQ problems such as voltage sag and unbalance, current distortion, and low power factor exist in power systems. Voltage sag and unbalance are generally caused by faults in power systems [77]. Current distortion is caused by non-linear loads in power systems [77]. Low power factor is caused by the use of large number of inductive loads [77]. To solve these PQ problems, voltage sag compensators, and local harmonic current and reactive power compensators are used [78][80]. A short description of major PQ problems is given in the following sub-sections. 


\subsubsection{Voltage Disturbances}

It is desirable to have a sinusoidal AC voltage with fixed amplitude and frequency [76]. There are different factors which can disturb this voltage. Electrical currents when flowing through distribution networks produce voltage drop along these networks [76]. In case the power demand of a load is very high, the large current demanded by this load may result in large voltage drop in the line impedance and thus result in reduced load voltage [76].

In order to keep the frequency of the voltage constant in a power system, it is essential to have a good balance between the generated and consumed power [76]. But the power consumed by the customers will vary in different ways. Thus the generation units have to meet the generation-load balance minute-by-minute in order to maintain system frequency at a constant value [76].

Some other reasons for voltage disturbances can be due to inevitable transient and proliferation of non-linear loads [76]. Voltage quality improvement can be done at utility side by installing voltage sag compensators at utility side [80]. Also, by installing current harmonic compensators at load side, the non-linear harmonic load currents can be compensated [80]. Thus, maintaining voltage quality is the responsibility of both the utility and consumers [77].

\subsubsection{Frequency Variations in Voltage}

Network frequency is stable most of the time in power systems [76]. If a large imbalance between generation and demand occurs, then system frequency will be altered [76], [77]. For example, in Spain frequency varies only by $0.1 \mathrm{~Hz}$ for a very large load change of $12 \mathrm{GW}$ [76]. Maintaining system frequency is important aspect as frequency variations affect the rotating machines, clocks and electronics equipments, which use frequency as their reference value [76].

\subsubsection{Voltage Fluctuations}

Voltage fluctuations are variations in the magnitude of the supply voltage by $\pm 10 \%$ of the nominal value for a duration lasting several milliseconds to $10 \mathrm{~s}$ [76]. Voltage fluctuations are caused due to variations in loads and changes in network conditions such as connection of arc furnaces, welding equipments, large induction motors, etc. [76], [77]. Small voltage fluctuations can cause flickering of lamps, which cause inconvenience to consumers [77]. 


\subsubsection{Voltage Dip or Sag}

As per EN50160 standard, a voltage dip or sag is defined as the reduction of voltage to a value between $1 \%$ and $90 \%$ of the rated voltage for a short time duration ranging from $10 \mathrm{~ms}$ to 1 min [81]. Voltage dip depth is defined as the difference between the minimum rms value during the duration of the dip and the rated voltage [76]. A voltage dip which causes reduction of voltage from the rated value to around $1 \%$ of the rated value is called as a short voltage interruption [76]. The causes for voltage dips and interruptions are short-circuits, malfunctions in the power system and sudden switching of large loads such as induction motors and generators [76], [77].

\subsubsection{Voltage Imbalance}

Voltage imbalance is a condition in which the phase voltages in the three-phase system differ in magnitude or in which the phase difference between one phase voltage and the other phase voltage is not the usual $120^{\circ}$ [77]. Numerically voltage imbalance can be defined as the maximum deviation from the average of all the three phase voltages, expressed in percent [77].

Different causes of voltage imbalance are due to reasons such as presence of single-phase loads in a three-phase circuit, single-phasing conditions, blown fuses in one or more phases of a three-phase capacitor bank, etc. [76], [77]. Usually, due to the presence of single-phase loads in a three-phase system, voltage imbalance of 1\%-2\% occurs and higher voltage imbalance of around 5\% can occur due to single-phasing conditions [76].

\subsubsection{Harmonics}

Due to proliferation of non-linear loads in power systems, harmonic issues are of serious concern [76]. Non-linear loads draw harmonic currents from the power supply thereby distorting the source current [76]. In the distribution line impedances, these harmonic currents cause harmonic voltage drops resulting in the harmonic distortions of load voltages [76]. Thus the system designer should consider different design issues and power quality improvement methods in power systems to reduce or eliminate the voltage and current harmonic distortions [76]. IEEE Standard 519-2014 specifies the recommended power quality standards for the operation of power systems containing both linear and non-linear loads [82]. 


\subsection{Power Quality Improvement Methods}

Various methods have been proposed for harmonic current and reactive power compensation [80]. Basic principles of compensation were developed in 1970s with the introduction of active power filters (APFs) [76]. APFs are inverter circuits consisting of semiconductor switches such as thyristor and IGBT, which are controlled to operate as harmonic current or voltage sources [76]. The development in power electronics topologies and control technologies led to the widespread use of APFs [76]. These devices are capable of fully compensating voltage unbalances, reactive power of loads and harmonic currents of nonlinear loads [76], [80]. APFs are also called as active power line conditioners (APLC) because they can be installed in distribution lines to modify the power delivered to the loads by different compensation methods [76]. Different kinds of APLCs are shunt filters [76], [83]-[85], series filters [76], [86]-[88] and hybrid filters [76], [89]-[93].

\subsubsection{Shunt Active Filter}

APF connected in shunt or parallel with the loads which is capable of injecting the required compensating harmonic current is called shunt APF [76], [93]. This filter injects a harmonic current which is equal and opposite to the harmonic current injected by the non-linear load [76]. Thus the source current becomes sinusoidal and free of harmonics [76]. Shunt APF is shown in Figure 2-14. One main advantage of shunt APF over a passive filter is that the source impedance does not affect the filtering characteristics of a shunt APF [76]. Also, the shunt APF provides better compensation characteristics than a parallel passive filter [93]. In addition, unlike in a passive filter, frequency variations and tolerances in filter components do not affect the compensation characteristics of a shunt APF [76].

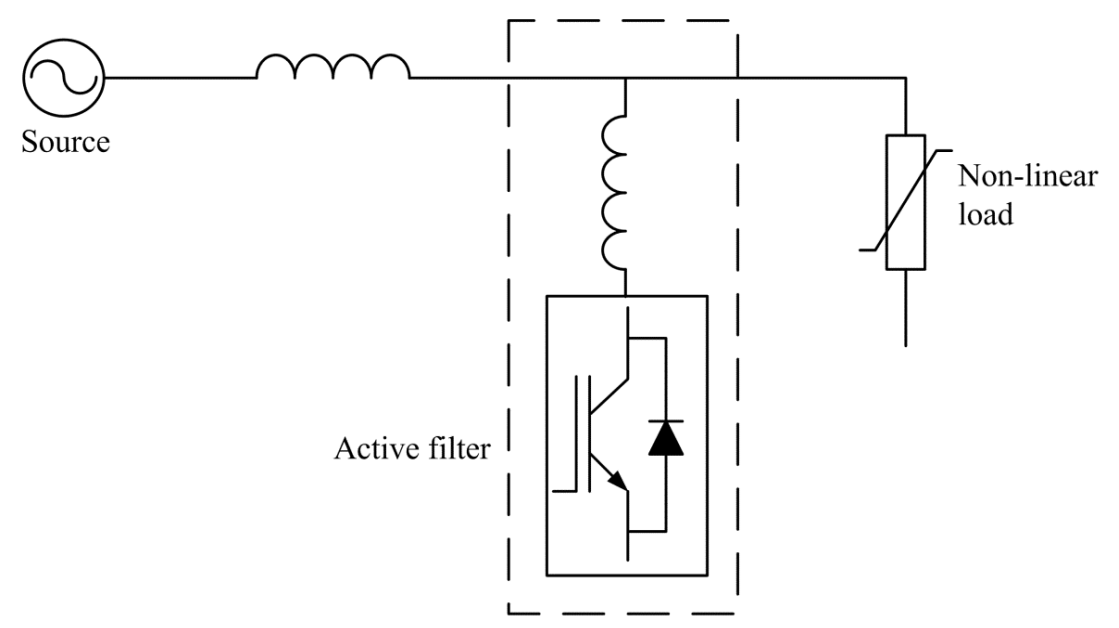

Figure 2-14: Shunt active filter [76]. 


\subsubsection{Series Active Filter}

In this filter, the inverter circuit is connected in series with the load to be compensated as shown in Figure 2-15. In this arrangement, the series inverter injects an appropriate voltage of desired magnitude and frequency to mitigate the sag, unbalance, swell and distortion in the supply voltage [76], [87]. As a result a sinusoidal load voltage of required magnitude and frequency is obtained. This type of series active filter configuration is also called as dynamic voltage restorer (DVR) [87], [88]. DVR is used commonly in industries to protect sensitive loads from voltage disturbances [76].

One disadvantage of this DVR is that it includes additional impedance and losses in the distribution system because of the coupling transformer used to connect DVR to distribution systems [76]. Under normal operating conditions, DVR operates below its rated capacity [76]. Thus, it is beneficial to include other additional functions in DVR to improve its utilization capacity [76].

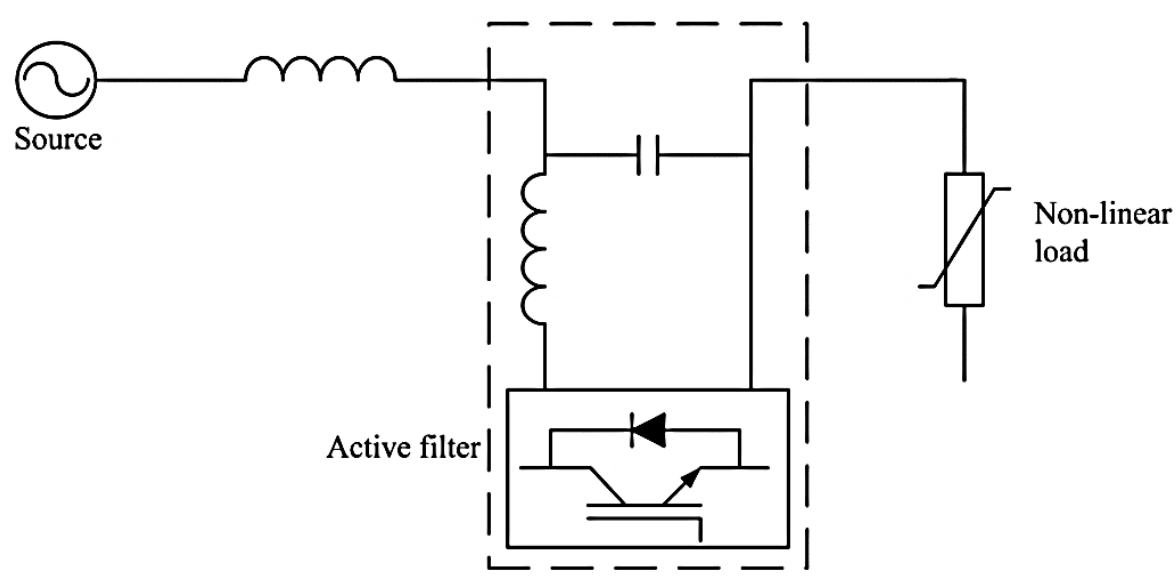

Figure 2-15: Series active filter [76].

\subsubsection{Hybrid Active Filters}

Different topologies having different combinations of series active and passive filters, and shunt active and passive filters have been proposed in literature [76], [89]-[93]. The most commonly used and cited in literature is only discussed here. In Figure 2-16, a parallel combination of an active filter and a passive filter is shown [76]. In this hybrid filter topology, the passive filter removes most of the significant harmonics contained in the nonlinear load, thus reducing the power rating needed for the active filter [76]. Some of the drawbacks of this topology are that its filter characteristics are influenced by the source impedance, and series and parallel resonance may appear between source impedance and 
parallel passive filter at certain frequencies [76]. In Figure 2-17, another hybrid filter consisting of a series active filter and a shunt passive filter is shown [76]. In this filter, the series active filter removes the harmonics, unbalance and distortions in the supply voltage and thus provides a clean sinusoidal voltage at the load side [76]. The shunt passive filter acts as the harmonic current compensator [76].

In Figure 2-18, another hybrid filter is shown. This filter consists of an active filter connected in series with a passive filter and this combination is connected in parallel with the non-linear load [76], [89]. This configuration helps in the improvement of filtering characteristics of the passive filter by providing an active filter [89]. Thus an enhanced harmonic current and reactive power compensator can be obtained with this hybrid filter which is better than the hybrid filter shown in Figure 2-16 [76].

Several different configurations of shunt and series filters have been studied in order to improve the rated power, performance characteristic and dynamic response of APFs [90]. Finally, another important combination of shunt and series active filters is shown in Figure 219. In this filter, two active filters are present, one connected in series with the non-linear load and another connected in shunt with the non-linear load [90], [93]. The series filter mitigates the voltage disturbances originating in the source and thus provide a clean sinusoidal voltage waveform at the load side [76]. The shunt active filter injects the compensating current, consisting of required harmonic components and thus makes the source current sinusoidal [76]. This hybrid filter improves the quality of both the voltage and current, so it is called as a universal active filter or a unified power quality conditioner (UPQC) [76]. The main disadvantage of UPQC is its high cost [76]. Several configurations of UPQC and its applications in microgrid systems have been studied in [94]-[98].

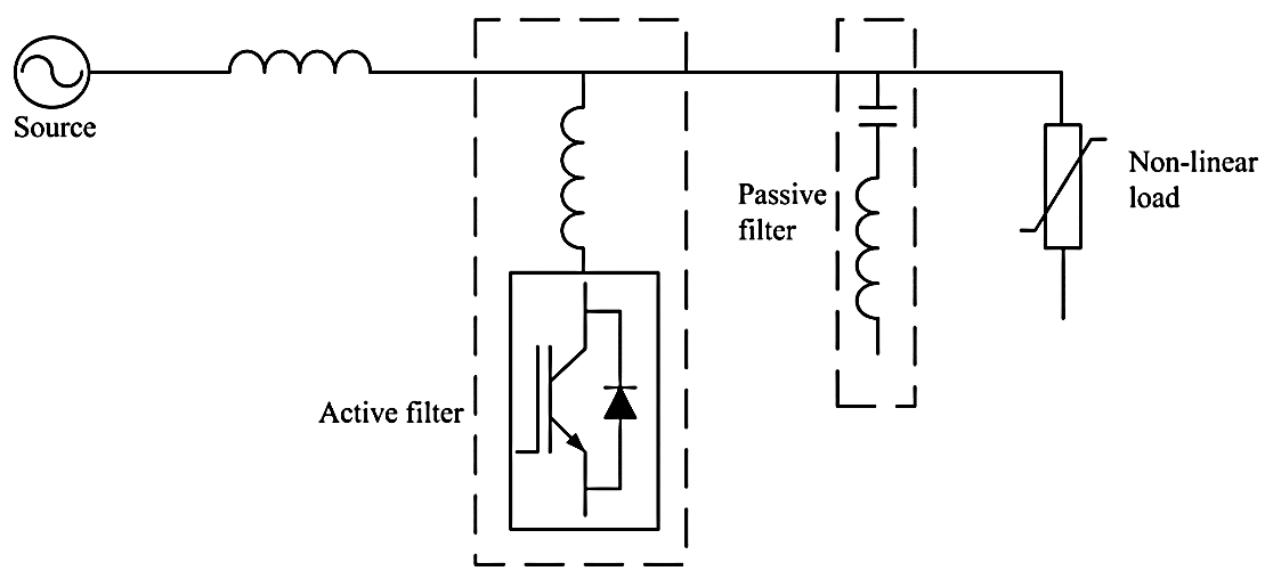

Figure 2-16: Parallel combination of an active filter and a passive filter [76]. 


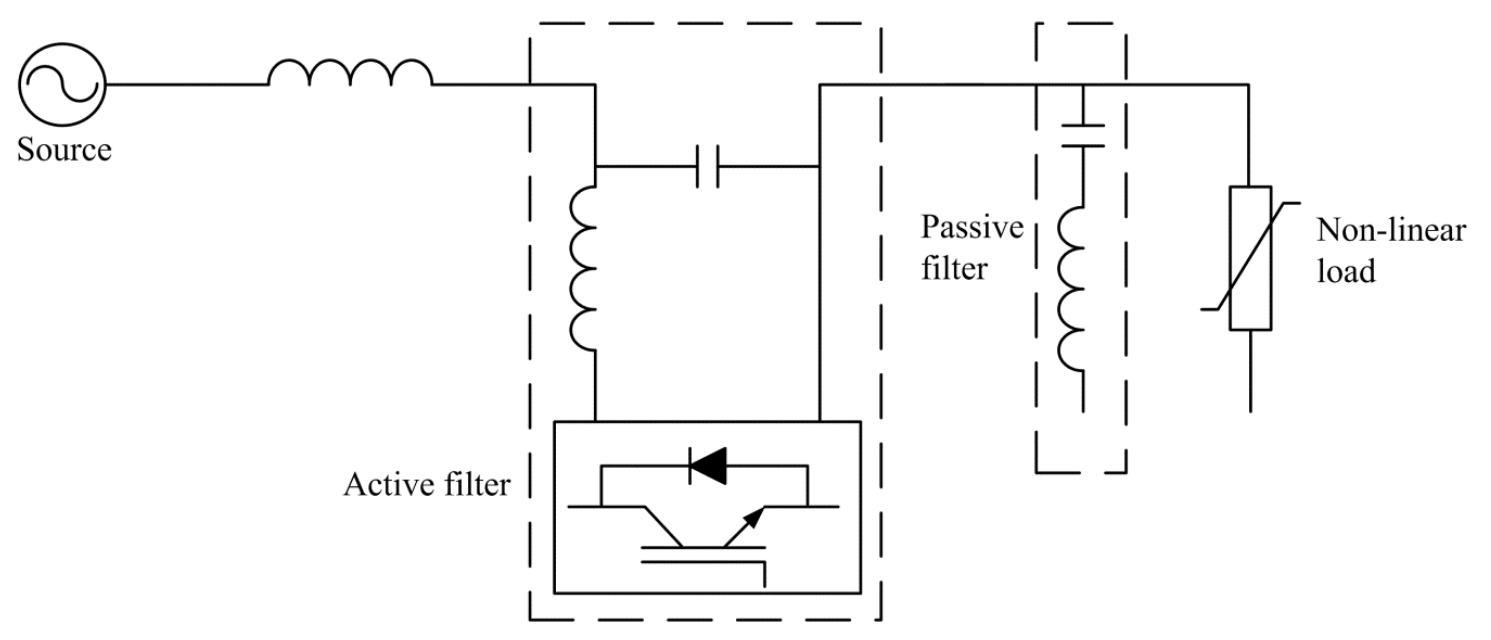

Figure 2-17: Hybrid topology with series active filter and shunt passive filter connected with the load [76].

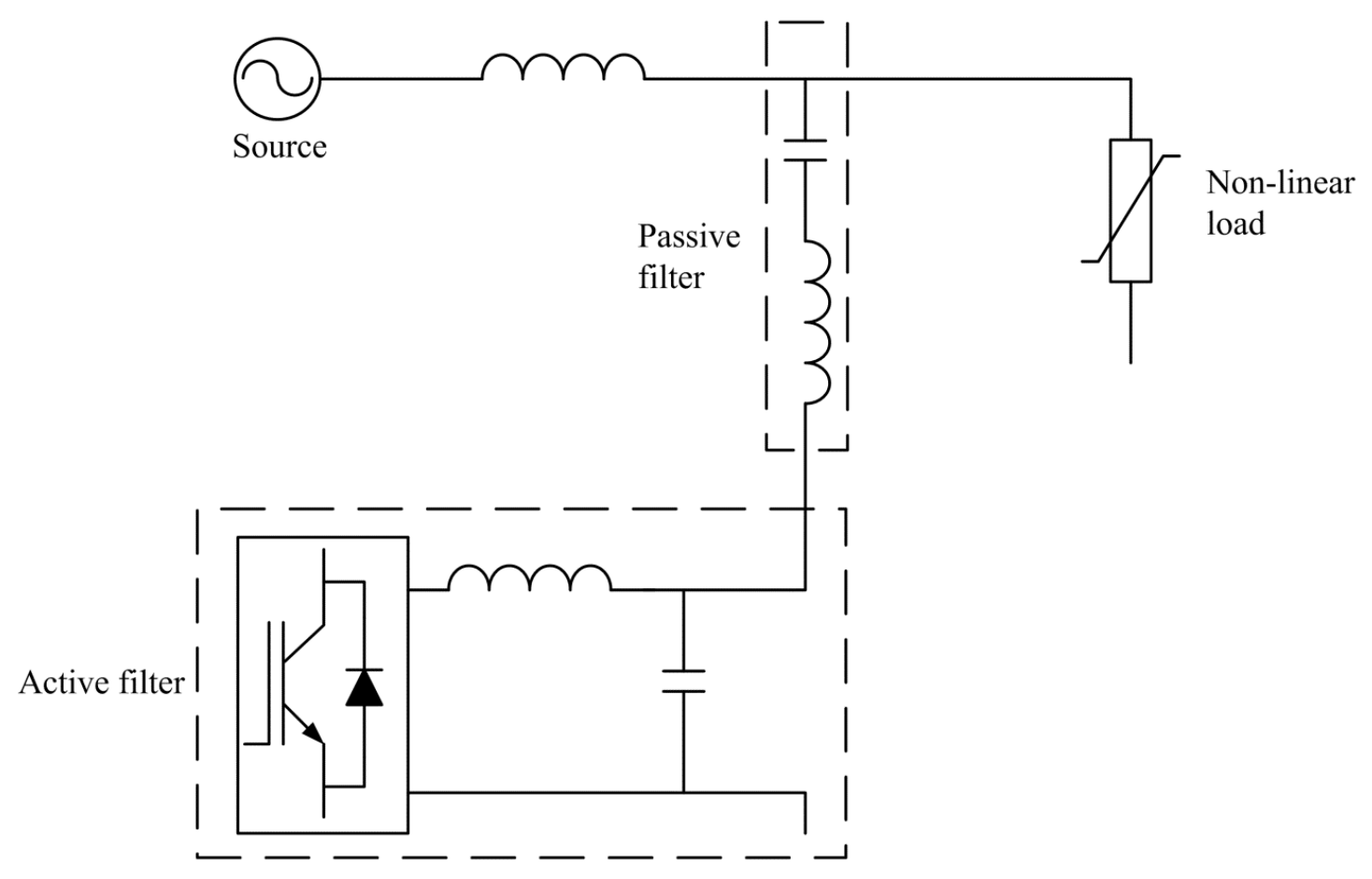

Figure 2-18: Combined topology of series active and passive filters, in parallel with the load [76]. 


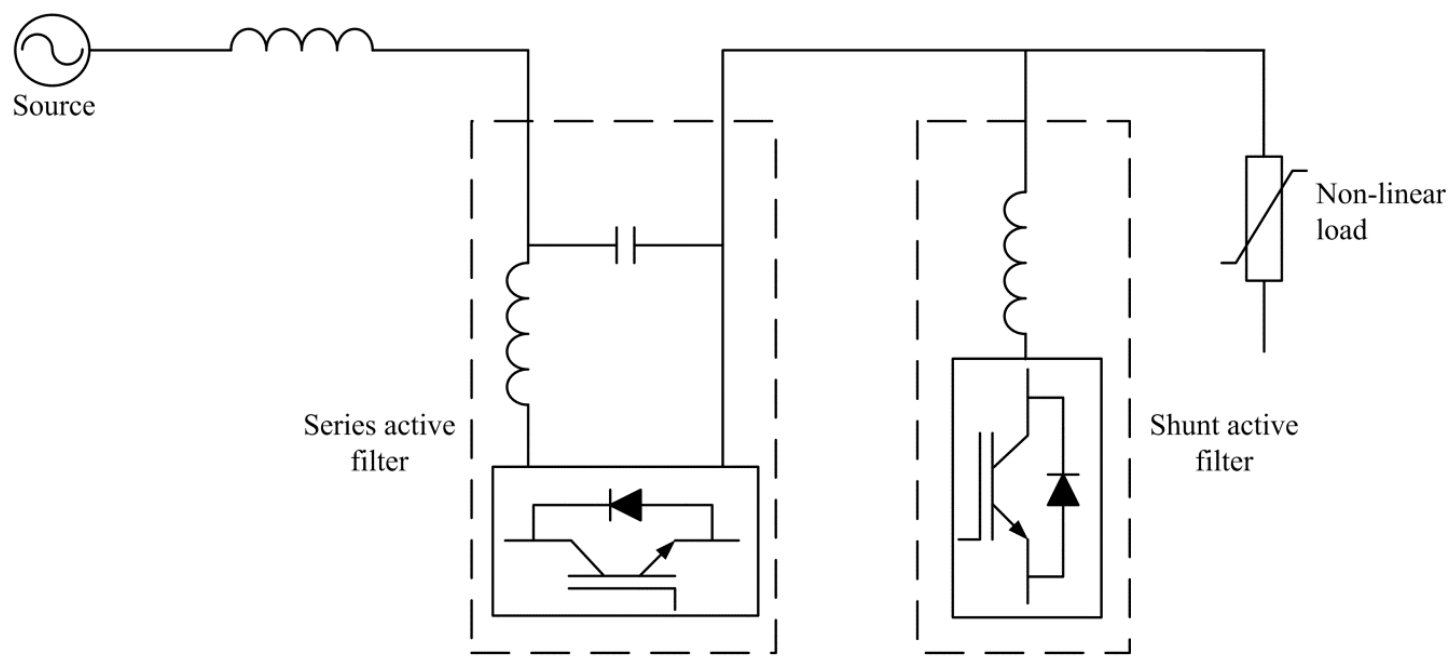

Figure 2-19: Unified power quality conditioner [76].

\subsubsection{Local and Non-local Compensators}

Depending on the position of the harmonic current and reactive power compensators with respect to the loads in a power system, two different methods of compensation are studied, namely local and non-local compensation. In local compensation, the compensators are located close to the loads in different filter topologies explained in the sub-sections above. But, in case of non-local compensation, compensators can be installed at some distance away from the loads and therefore the impedance of the interconnecting line between the compensator and the loads must be taken into consideration while devising an effective nonlocal compensation method. Even though local harmonic current and reactive power compensation methods are widely available in literature, non-local compensation methods, based on their several advantages, are alternatives to be necessarily considered in the future for large MMG and distribution systems, which consist of widely dispersed loads.

Non-local harmonic current and reactive power compensation are relatively new areas open to research. In [99], optimization is performed for an active filter installed remotely away from the distorting load to minimize the size of the active filter but it does not discuss about non-local harmonic current compensation method. In [100], the optimal size of the non-local reactive power compensation system is only determined to reduce the cost of capacitors and it does not discuss any method for implementing the non-local reactive power compensation. So, in these research works, the explanation about the implementation of non-local harmonic current and reactive power compensation is not given. In Chapter 5 of this thesis, a new method of non-local harmonic current and reactive power compensation in a MMG system 
with the use of a SSND is proposed. The advantages of non-local harmonic current and reactive power compensation using a single device over local harmonic current and reactive power compensation are as follows:

- It will eliminate the burden of local harmonic current and reactive power compensation using DG and battery inverters in microgrid systems and thus reduce the size and cost of these DG and battery inverters.

- It allows the expansion of the non-local compensating system to meet any future load increase [100].

- It allows the use of the compensator independently from the loads in the system and thus compensating harmonic current and reactive power can be supplied to the loads located close to or far apart from the compensator [101].

- It is often believed to be cheaper because the single compensating device is less costly to purchase than multiple dispersed small compensating devices [102].

\subsection{Summary}

In the future, integration of multi-microgrids into distribution networks will increase rapidly due to liberalization of electricity market, global energy demands and technological advancement. In general, two types of multi-area MMG systems are proposed in this thesis. First type is AC/AC multi-area MMG system consisting of interconnected AC microgrids and the second type is AC/DC multi-area MMG system consisting of both $\mathrm{AC}$ and $\mathrm{DC}$ microgrids. These multi-area MMG systems combine the advantages of both microgrid and interconnected power systems and can serve as large power pools for meeting bulk power demands. To develop efficient methods of operation and control for these multi-area MMG systems, the existing state-of-art control schemes adopted in conventional MMG systems are reviewed in this chapter.

The definition of an effective control scheme is essential for the effective operation of the proposed multi-area MMG systems. The control scheme should take into account the different operating modes of the interconnected microgrids in the MMG system. Power exchange between the interconnected microgrids in the MMG system is also an important aspect which should be efficiently managed by this control scheme. Also, the interaction between the centralized and local controllers is crucial for the reliable operation of the multiarea MMG system. To maintain the stable operation of the MMG system during different operating modes such as grid-connected and islanded modes, an efficient local control 
technique for the DG and storage battery converters is essential. Thus, a state-space model based MPC method is chosen in this research to achieve optimal operation and performance of the MMG system. Different control methods including MPC are discussed in detail in this chapter. The advantages of MPC over other conventional control methods are also briefly explained in this chapter.

Power quality issues, although widely explored in single microgrid systems, is a relatively new research area to be undertaken in MMG systems. Several power quality issues and power quality improvement methods exist in single microgrid systems, which are discussed in detail in this chapter to understand their significance and relevance with respect to the MMG systems. Application of the power quality improvement methods adopted in single microgrid systems directly into the MMG systems may not yield the required results. Thus, an extensive research is needed to understand the impact of power quality issues and propose new methods for solving these issues in the MMG systems. Taking this view into consideration, an analytical background study is conducted in this thesis to understand the impact of power quality issues on the MMG systems and therefore a new method of nonlocal harmonic current and reactive power compensation is proposed. In this chapter, the methods of local and non-local compensation are explained and the advantages of non-local compensation over local compensation are also briefly discussed. 


\section{CHAPTER 3 OPERATION AND CONTROL OF AC/AC MULTI-AREA MMG SYSTEM}

\subsection{Introduction}

This chapter presents a generalized architecture of an AC/AC multi-area MMG system, consisting of interconnected AC microgrids. Also, a control method is proposed to regulate load voltage and system frequency during microgrid islanding in this MMG system. In the event of islanding of a microgrid from the distribution grid in the proposed MMG system, load voltage of the islanded microgrid and system frequency are affected. To overcome these problems, a control system for the MMG system is proposed. The proposed control system facilitates desired power exchange between grid-connected and islanded microgrids, and achieves effective voltage and frequency regulation in the MMG system. The main significance of the proposed MMG system is that multiple microgrids in different locations can be interconnected to meet larger bulk power demands. This ensures improved reliability and security of power supply in the MMG system. An improved MPC is used to regulate various parameters such as output voltage, frequency and power of the inverters in the MMG system. The proposed MMG system is tested during islanding and load shedding using simulation studies. The simulation studies show that the inverters can operate effectively using MPC to provide the desired voltage, frequency and power during islanding and load shedding.

\subsection{System Description}

The proposed generalized architecture of an AC/AC multi-area MMG system is shown in Figure 3-1. It consists of ' $n$ ' number of microgrids, each of them separated from the others by interconnecting lines of about $10 \mathrm{~km}$. The minimum value of ' $n$ ' is 3 . The microgrids are interconnected with each other through lines $1,2, \ldots, n$. It is assumed that the main distribution grid is sectionalized into distribution grid 1, distribution grid 2, .., distribution grid $n$. Each microgrid is connected to its respective distribution grid through a $0.4 / 22 \mathrm{kV}$ distribution transformer. Voltages at the end of the lines are represented by $V_{i} \angle \delta_{i}(i=1,2, \ldots$, $n)$. Line resistances and inductances are represented as $R_{t i}$ and $L_{t i}$ respectively. The sending end real and reactive power flows through the lines are represented as $P_{s i}$ and $Q_{s i}$. The receiving end real and reactive power flows through the lines are represented as $P_{r i}$ and $Q_{r i}$. 
Each microgrid in the proposed MMG system basically operates in two different modes of operation: (1) grid-connected mode when the microgrid is connected to its respective distribution grid; and (2) islanded mode when the microgrid is disconnected from its respective distribution grid. The schematic diagram of microgrid $i(i=1,2, \ldots, n)$ used in the proposed MMG system is shown in Figure 3-2. It consists of a DG unit, a storage battery unit and an AC load. The DG unit is connected to the load bus through a DC/DC boost converter and a DG DC/AC inverter. The storage battery unit is connected to the load bus through a bidirectional DC/DC converter and a battery DC/AC inverter. The load bus voltage $i$ is represented as $V_{L i} \angle \delta_{i}$. The real and reactive power outputs of DG inverter $i$ are represented as $P_{d i}$ and $Q_{d i}$ respectively. The real and reactive power outputs of battery inverter $i$ are represented as $P_{b i}$ and $Q_{b i}$ respectively. The inverter can operate in two different modes: the first mode is CCM where inverter's output current is regulated to the desired value, and the second mode is VCM where inverter's output voltage is regulated to the desired value.

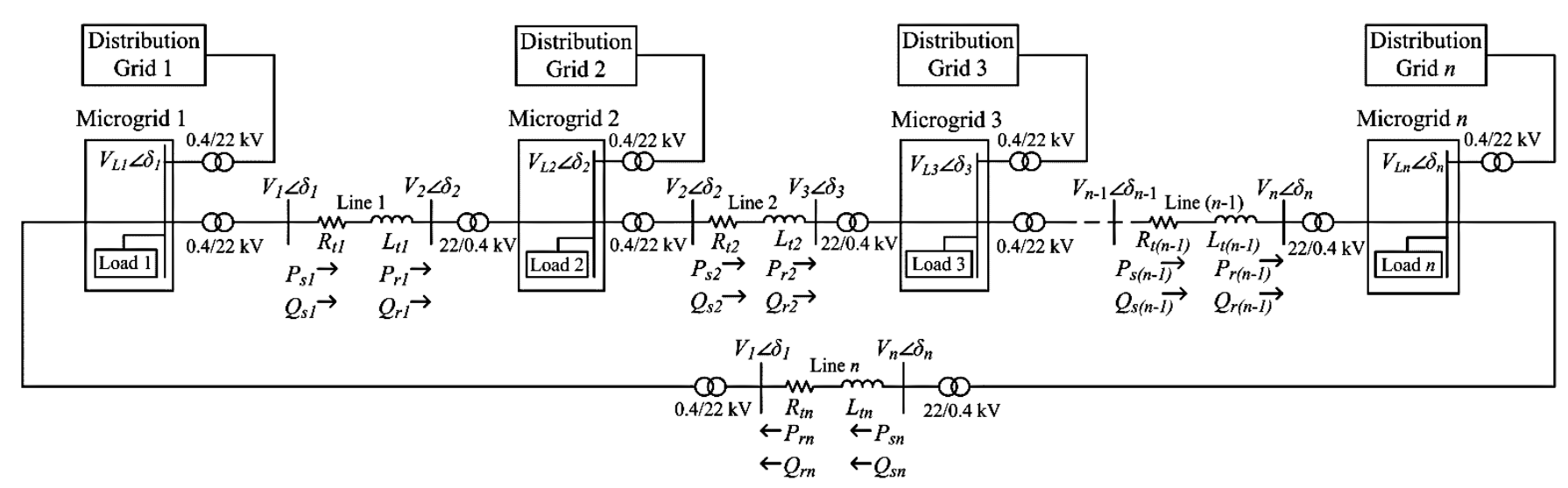

Figure 3-1: Proposed generalized architecture of an AC/AC multi-area MMG system.

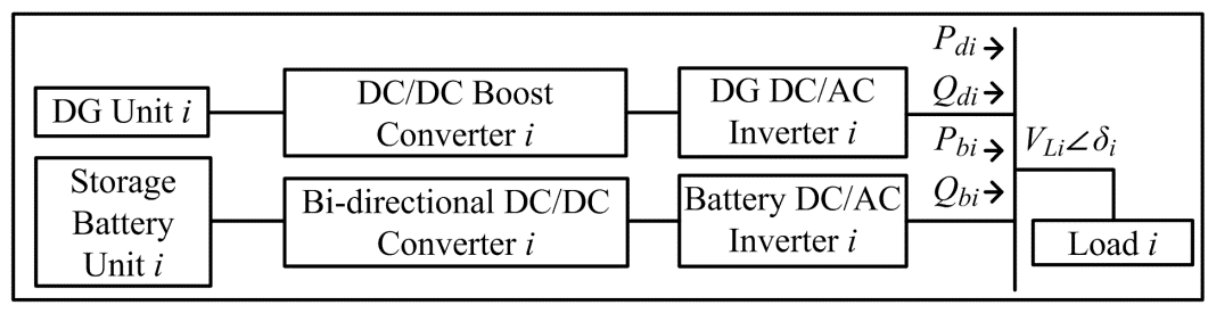

Figure 3-2: Configuration of microgrid $i$.

During grid-connected operation of a microgrid in the MMG system, the distribution grid regulates the load bus voltage and frequency, and thus both DG and battery inverters in the microgrid operate in CCM. In the islanded mode of a microgrid in the MMG system, the battery and DG inverters in the microgrid act as the master and slave inverters respectively 
[64]. During islanded operation, the battery inverter operates in VCM and DG inverter operates in CCM. In the MMG system, the principle of control of power flows at the sending and receiving ends of the lines connected between the adjacent microgrids for $n \geq 3$ is based on the generalized power flow equations (3.1) - (3.4) [103] as follows:

If $V_{i}=V$, then

$$
\begin{aligned}
P_{s i} & =\left(V^{2} / Z_{t i}\right) \cos \theta_{t i}-\left(V^{2} / Z_{t i}\right) \cos \left(\theta_{t i}+\delta_{i, i+1}\right) \text { for } i=1,2, \ldots, n-1 \\
& =\left(V^{2} / Z_{t i}\right) \cos \theta_{t i}-\left(V^{2} / Z_{t i}\right) \cos \left(\theta_{t i}+\delta_{i, 1}\right) \text { for } i=n \\
P_{r i} & =\left(V^{2} / Z_{t i}\right) \cos \left(\theta_{t i}-\delta_{i, i+1}\right)-\left(V^{2} / Z_{t i}\right) \cos \theta_{t i} \text { for } i=1,2, \ldots, n-1 \\
& =\left(V^{2} / Z_{t i}\right) \cos \left(\theta_{t i}-\delta_{i, 1}\right)-\left(V^{2} / Z_{t i}\right) \cos \theta_{t i} \text { for } i=n \\
Q_{s i} & =\left(V^{2} / Z_{t i}\right) \sin \theta_{t i}-\left(V^{2} / Z_{t i}\right) \sin \left(\theta_{t i}+\delta_{i, i+1}\right) \text { for } i=1,2, \ldots, n-1 \\
& =\left(V^{2} / Z_{t i}\right) \sin \theta_{t i}-\left(V^{2} / Z_{t i}\right) \sin \left(\theta_{t i}+\delta_{i, 1}\right) \text { for } i=n \\
Q_{r i} & =\left(V^{2} / Z_{t i}\right) \sin \left(\theta_{t i}-\delta_{i, i+1}\right)-\left(V^{2} / Z_{t i}\right) \sin \theta_{t i} \text { for } i=1,2, \ldots, n-1 \\
& =\left(V^{2} / Z_{t i}\right) \sin \left(\theta_{t i}-\delta_{i, 1}\right)-\left(V^{2} / Z_{t i}\right) \sin \theta_{t i} \text { for } i=n
\end{aligned}
$$

where $\delta_{i, i+1}=\delta_{i}-\delta_{i+1}$ for $i=1,2, \ldots, n-1$

$=$ phase angle difference between voltages $V_{i}$ and $V_{i+1}$

$\delta_{i, 1}=\delta_{i}-\delta_{1}$ for $i=n$

$=$ phase angle difference between voltages $V_{i}$ and $V_{l}$

$\delta_{i}=$ phase angle of voltage $V_{i}$ for $i=1,2, \ldots, n$

$Z_{t i} \angle \Theta_{t i}=$ impedance of line $i$

$R_{t i}=$ resistance of line $i$

$X_{t i}=$ reactance of line $i$

For satisfactory operation of the MMG system, the voltage magnitude at each load bus is maintained at the same nominal value [103]. Thus, the control of real power flows at the sending and receiving ends of the interconnecting lines between adjacent microgrids in the 
MMG system is achieved primarily through the variations in phase angles of load bus voltages.

\subsection{Modelling and Control of Inverters in the MMG System}

MPC is a promising control algorithm for the control of inverters in the MMG system due to its many advantages. As a digital control algorithm, MPC is suitable for power electronic devices [36]. In addition, it does not require additional voltage and current loops, modulation and coordinate transformations [36]. The control performance of the Finite Control Set MPC method introduced in [36] depends on system parameters such as grid, line and load impedances whereas the performance of the proposed MPC algorithm is independent of grid, line and load impedances. The proposed MPC algorithm can thus be used for multiple inverters for different system parameters without modification of the state-space model of the inverters. Therefore the main advantage of the proposed MPC algorithm is the scalability and implementation for multiple inverter modules in a large MMG system. As the DG and battery inverters work in different operating control modes, it is crucial for each microgrid in the MMG system to remain stable during its grid-connected and islanded modes of operation. The proposed MPC algorithm is chosen in this research to achieve optimal performance of the MMG system. The model of DG and battery inverters in the MMG system is shown in Figure 3-3. The DC/DC converters stabilize the input voltage $V_{d c}$ of the DG and battery inverters. For the three-phase inverter model, the switching states $\left(S_{i 1}-S_{i 6}\right)$ of the switches have only binary values " 1 " and " 0 " in a closed state and an open state respectively. The lower switches have the complementary values of their upper switches. Thus, the switching signals of each phase are defined as follows:

$$
S_{a}=S_{i 1}=\bar{S}_{i 2}, S_{b}=S_{i 3}=\bar{S}_{i 4}, S_{c}=S_{i 5}=\bar{S}_{i 6}
$$

The switching signal space vector [36] can be defined as

$$
S=\frac{2}{3}\left(S_{a}+\alpha S_{b}+\alpha^{2} S_{c}\right) \text { where } \alpha=e^{j(2 \pi / 3)} .
$$

The three-phase switching voltage vector $v_{s}[36]$ can be expressed as

$$
v_{s}=V_{d c} S
$$


Totally eight possible values for $v_{s}$ can be obtained for eight possible combinations of $S_{a}, S_{b}$ and $S_{c}$ [36]. By applying Kirchhoff's voltage and current laws to the inverter model in Figure 3-3, the following equations are obtained:

$$
\begin{gathered}
v_{s}=i_{d} R_{l}+L_{f}\left(d i_{d} / d t\right)+v_{0} \\
i_{d}=C_{f}\left(d v_{0} / d t\right)+i_{0}
\end{gathered}
$$

where $R_{l}$ represents the internal loss of the inverter, $L_{f}$ and $C_{f}$ are the inductance and capacitance of the LC filter respectively, $i_{d}$ is the three-phase current which flows through $L_{f}$, $i_{0}$ is the three-phase output current, and $v_{0}$ is the three-phase output voltage. Due to high sampling frequency, the following assumption can be made:

$$
d i_{0} / d t=0
$$

By rearranging and simplifying (3.7)-(3.10) into a state-space form and by using Euler difference method, the discrete state-space model of the inverter is obtained as follows:

$$
\begin{gathered}
x(k+1)=A_{d} x(k)+B_{d} u(k) \\
y(k)=C_{d} x(k)
\end{gathered}
$$

where $x(k)=\left[\begin{array}{lll}i_{d}(k) & v_{0}(k) & v_{0}^{\prime}(k)\end{array}\right]^{T}, y(\mathrm{k})=\left\{\begin{array}{c}i_{0}(\mathrm{k}) \text { in CCM } \\ v_{0}(\mathrm{k}) \text { in } \mathrm{VCM}\end{array}, u(\mathrm{k})=v_{s}\right.$

The discretized coefficients $A_{d}, B_{d}$ and $C_{d}$ are as follows:

$$
\begin{aligned}
A_{d} & =\left[\begin{array}{ccc}
1-\left(R_{l} T_{s} / L_{f}\right) & -T_{s} / L_{f} & 0 \\
0 & 1 & T_{s} \\
-T_{s} R_{l} /\left(L_{f} C_{f}\right) & -T_{s} /\left(L_{f} C_{f}\right) & 1
\end{array}\right], B_{d}=\left[\begin{array}{c}
T_{s} / L_{f} \\
0 \\
T_{s} /\left(L_{f} C_{f}\right)
\end{array}\right], \\
C_{d} & = \begin{cases}{\left[\begin{array}{lll}
1 & 0 & -C_{f}
\end{array}\right] \text { in CCM }} \\
{\left[\begin{array}{lll}
0 & 1 & 0
\end{array}\right] \text { in VCM }}\end{cases}
\end{aligned}
$$

where $T_{s}$ is sampling time and $v_{0}^{\prime}=d v_{0} / d t$.

The state-space model of the inverter in (3.11) contain only parameters of the inverter such as power loss resistance, and filter inductance and capacitance and do not contain any system parameters such as grid, line, and load impedances. Thus the proposed MPC algorithm, unlike the existing MPC algorithms, is independent of grid, line and load impedances of the system considered. The overall state-space power system model of the AC/AC multi-area MMG system obtained from compiling the differential equations of inverters, lines and loads 
is given in Appendix A. The MPC controller obtains the control signals by minimizing the following cost function which tracks the error between the reference variable $r(k)$ and the output variable $y(k)$ :

$$
J=[r(k)-y(k)]^{T}[r(k)-y(k)]+[r(k+1)-y(k+1)]^{T}[r(k+1)-y(k+1)]
$$

This cost function is evaluated for the predicted values of the variables to be controlled for each possible switching state. Then the state that minimizes the cost function is selected. The reference $r(k)$ is equal to the output current of the inverter in CCM and output voltage of the inverter in VCM. Thus MPC uses the state-space model of the inverter to control the output current of the inverter during CCM and output voltage of the inverter during VCM. In [36], the reference variable $r(k)$ is used to determine the control signal $u(k)$. In this chapter, two steps of reference are considered in the cost function to properly choose the control signal $u(k)$ and ensure an improved control performance in comparison with that in [36].

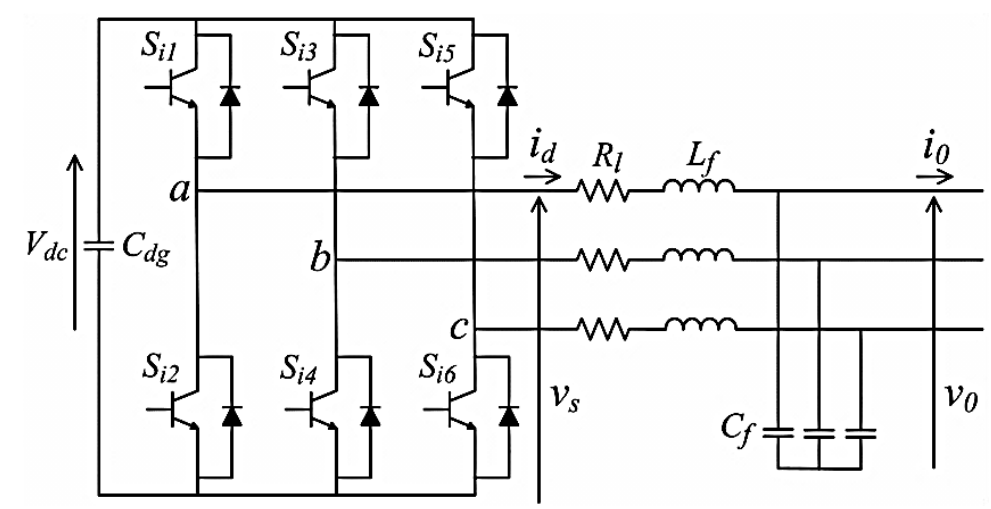

Figure 3-3: Three-phase inverter model.

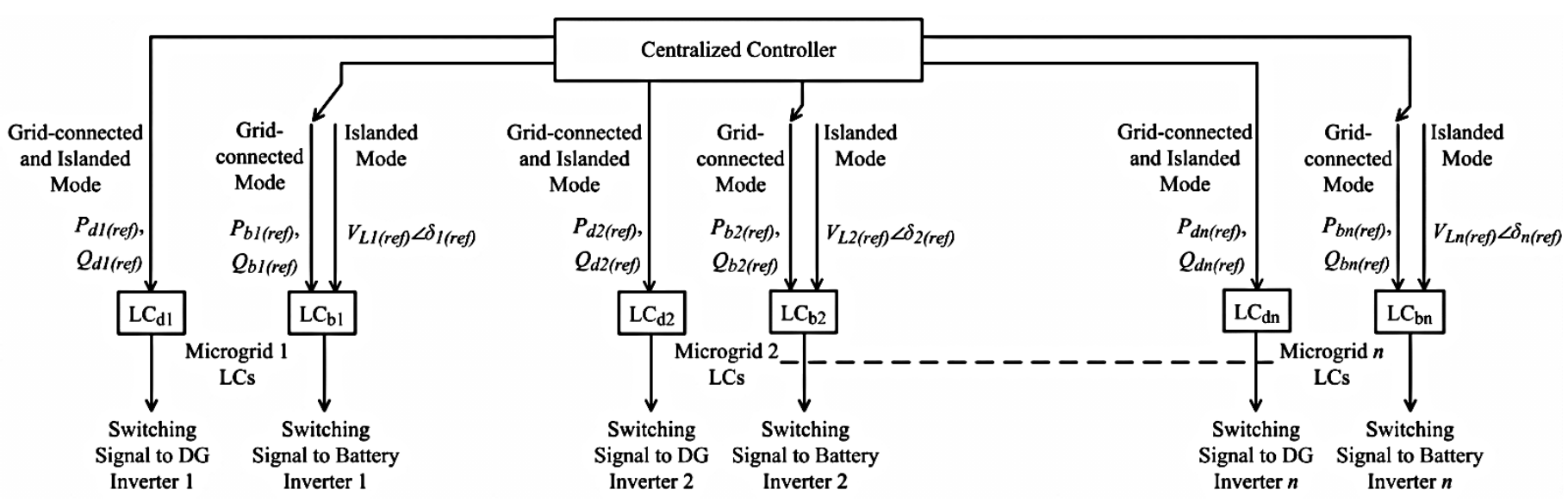

Figure 3-4: Proposed control system for AC/AC multi-area MMG system. 
The proposed control system for the multi-area MMG system is shown in Figure 3-4. It consists of a centralized controller for the overall MMG system and local controllers (LCs) for the DG and battery inverters in each microgrid. The centralized controller collects system data such as real and reactive power supplied by each distribution grid to its respective microgrid, load demands in the microgrids, operation modes of the microgrids, state of charge of the storage battery units, system frequency, etc. Based on these data, the centralized controller performs two main functions: (1) send the reference real and reactive power output signals, $P_{d i(r e f)}, Q_{d i(r e f)}(i=1,2, \ldots, n)$ and $P_{b i(r e f)}, Q_{b i(r e f)}$ to the DG and battery inverters' LCs respectively in the grid-connected mode of different microgrids; and (2) send the reference load bus voltage signals $V_{L i(r e f)} \angle \delta_{i(r e f)}$ to the battery inverters' LCs in the islanded mode of different microgrids. Using the proposed MPC technique, local controllers $\mathrm{LC}_{\mathrm{di}}$ and $\mathrm{LC}_{\mathrm{bi}}$ generate and send the optimal switching signals to the DG and battery inverters respectively. Thus there is an effective coordination among the centralized and local controllers to maintain the desired power flows between the microgrids in the MMG system. Also, a reliable and suitable high-speed communication network between centralized and local controllers is essential for effective operation of the MMG system [104].

During grid-connected mode of a microgrid in the MMG system, the DG inverter in the microgrid is controlled to deliver a predetermined amount of real power to the load. Battery inverter serves as the back-up inverter during grid-connected mode. Also, the DG inverter in the microgrid is controlled to deliver all of the reactive power required by the load in order to achieve unity power factor at the distribution grid side. During islanding of a microgrid in the MMG system, centralized controller detects a frequency drop in the system and sends an appropriate voltage reference signal to the battery inverter's LC of the islanded microgrid, as shown in Figure 3-4, to maintain the load bus voltage and desired power flows between the islanded microgrid and its adjacent grid-connected microgrids. The magnitude of the load bus voltage in the grid-connected or islanded microgrid is always maintained at the nominal value of $230 \mathrm{Vrms}$ (phase). The phase angle of the load bus voltage in the islanded microgrid is calculated based on the desired real power to be obtained at the receiving ends of the lines adjacent to the islanded microgrid. This phase angle is calculated from (3.2) as follows:

$$
\delta_{i, i+1(r e f)}=\theta_{t i}-\cos ^{-1}\left[P_{r i(r e f)} Z_{t i} / V^{2}+\cos \theta_{t i}\right] \text { for } i=1,2, \ldots, n-1
$$




$$
\delta_{i, 1(r e f)}=\theta_{t i}-\cos ^{-1}\left[P_{r i(r e f)} Z_{t i} / V^{2}+\cos \theta_{t i}\right] \text { for } i=n
$$

where $\delta_{i, i+1(\text { ref })}$ is the reference phase angle difference between $V_{i}$ and $V_{i+1}, \delta_{i, 1(\text { ref })}$ is the reference phase angle difference between $V_{i}$ and $V_{l}, P_{r i(r e f)}$ is the reference receiving end real power to be maintained in line $i$. The corresponding sending end real power flow in line $i$, and sending and receiving end reactive power flows in line $i$ are obtained by substituting the calculated value of the reference phase angle difference into (3.1), (3.3) and (3.4). Centralized controller continuously monitors the frequency of the MMG system and triggers underfrequency load shedding whenever the frequency deviation crosses the tolerance limit of $\pm 0.5 \mathrm{~Hz}$. The tolerance limit for frequency deviation adopted in Singapore is $\pm 0.5 \mathrm{~Hz}$ [105]. An underfrequency load shedding scheme is proposed for the MMG system as follows [103], [106]:

- $30 \%$ of non-sensitive load is shed in the islanded microgrid when the system frequency drops to $49.5 \mathrm{~Hz}$.

- $35 \%$ of additional non-sensitive load is shed in the islanded microgrid when the system frequency drops to $49 \mathrm{~Hz}$.

- The adjacent interconnecting lines of the islanded microgrid are tripped when the system frequency drops below $49 \mathrm{~Hz}$ and after a time delay of $0.1 \mathrm{~s}$ (response time of centralized controller), the remaining amount of non-sensitive load is shed in the islanded microgrid to restore the frequency of the islanded microgrid back to $50 \mathrm{~Hz}$. Intentional tripping of interconnecting lines is permitted if the system frequency drops below a low value [107].

\subsubsection{Flowcharts of the Proposed MPC Algorithm}

The flowcharts summarizing the procedure of the proposed MPC algorithm for voltage and current controls are given in Figure 3-5 and Figure 3-6 respectively. The flowchart in Fig. 3-5 summarizes the procedure of the proposed MPC algorithm for voltage control in the inverter. The inverter currents $i_{d}(k)$ and $i_{0}(k)$, and inverter voltage $v_{0}(k)$ are measured. From the discrete state-space model given in (3.11), the value of the predicted voltage $v_{0}(k+1)$ is obtained as follows: 


$$
v_{0}(k+1)=\frac{T_{S}}{C_{f}}\left[\left(1-\frac{R_{l} T_{S}}{L_{f}}\right) i_{d}(k)-\frac{T_{S}}{L_{f}} v_{0}(k)+\frac{T_{S}}{L_{f}} S V_{d c}-i_{0}(k)\right]+v_{0}(k)
$$

All the terms in (3.16) are defined in Section 3.3. The voltage $v_{0}(k+1)$ is calculated for eight possible values of $S$, which can be obtained for eight possible combinations of $S_{a}, S_{b}$ and $S_{c}$ [36]. The cost function $J$ is calculated by using the corresponding equation shown in Figure 3-5 for eight possible values of $S$. The value of $S$ which gives the minimum value of the cost function is chosen and the corresponding switching signals are generated and applied to the inverter [36].

The flowchart in Figure 3-6 summarizes the procedure of the proposed MPC algorithm for current control in the inverter. The inverter current $i_{d}(k)$ and inverter voltage $v_{0}(k)$ are measured. From the discrete state-space model given in (3.11), the value of the predicted current $i_{0}(k+1)$ is obtained as follows:

$$
i_{0}(k+1) \approx i_{d}(k+1)=\left(1-\frac{R_{l} T_{s}}{L_{f}}\right) i_{d}(k)-\frac{T_{s}}{L_{f}} v_{o}(k)+\frac{T_{s}}{L_{f}} S V_{d c}
$$

All the terms in (3.17) are defined in Section 3.3. The current $i_{0}(k+1)$ is calculated for eight possible values of $S$, which can be obtained for eight possible combinations of $S_{a}, S_{b}$ and $S_{c}$ [36]. The cost function $J$ is calculated by using the corresponding equation shown in Figure 3-6 for eight possible values of $S$. The value of $S$ which gives the minimum value of the cost function is chosen and the corresponding switching signals are generated and applied to the inverter. In both the given flowcharts, the outer loop is executed every sampling time and the inner loop is executed for each possible switching state, obtaining the optimal switching state to be applied during the next sampling period [36]. The detailed procedure for evaluation of cost function and selection of optimal switching state is given in Section 3.3.2. 


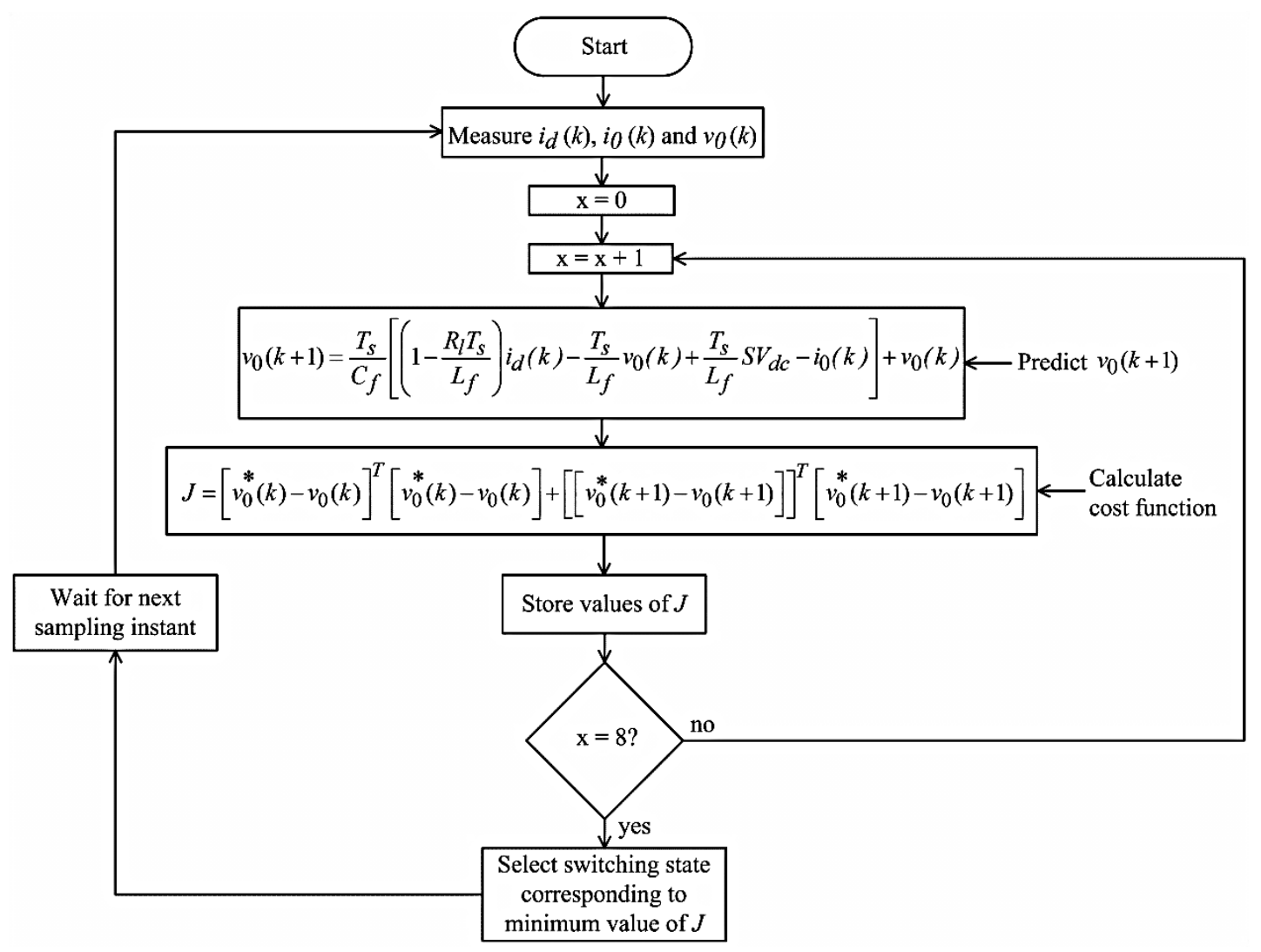

Figure 3-5: Flowchart of the proposed MPC algorithm for voltage control.

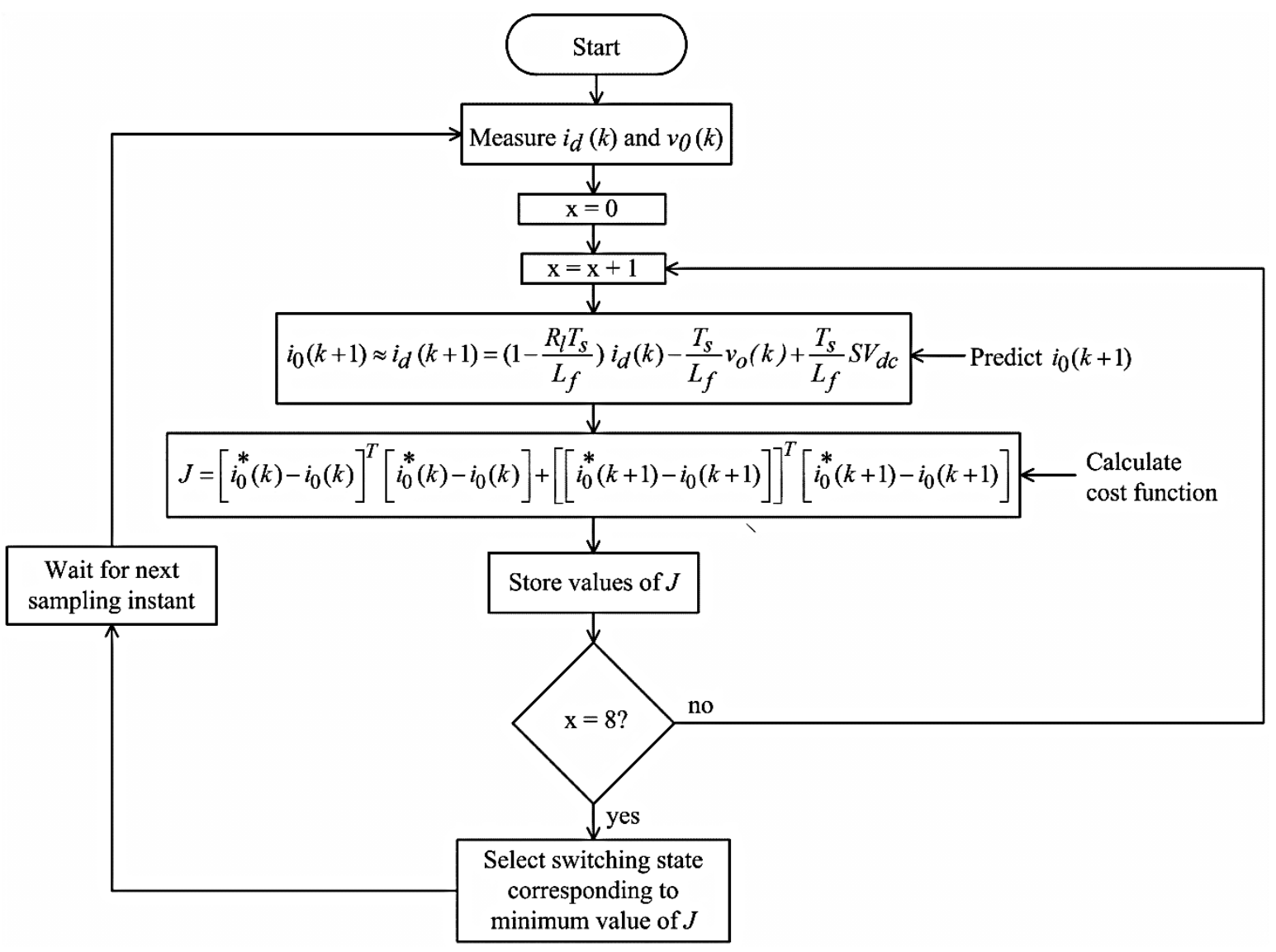

Figure 3-6: Flowchart of the proposed MPC algorithm for current control. 


\subsubsection{Procedures for Evaluation of Cost Function in the Proposed MPC Algorithm}

Procedures for evaluation of cost function and selection of optimal switching state in the proposed MPC algorithm for the three-phase inverter model consist of the following steps:

\section{1) Selection of cost function}

A cost function generally represents the error between the predicted variables and their references in the proposed MPC algorithm. The proposed MPC algorithm is used to control either the output currents or output voltages of the inverters in the MMG system. Thus the required cost function which is the same as given in (3.13) is given as follows:

$$
J=[r(k)-y(k)]^{T}[r(k)-y(k)]+[r(k+1)-y(k+1)]^{T}[r(k+1)-y(k+1)]
$$

The reference $r(k)$ is the reference output current of the inverter operating in CCM and reference output voltage of the inverter operating in VCM. Therefore in VCM, the cost function is given as

$$
J=\left[v_{0}^{*}(k)-v_{0}(k)\right]^{T}\left[v_{0}^{*}(k)-v_{0}(k)\right]+\left[\left[v_{0}^{*}(k+1)-v_{0}(k+1)\right]\right]^{T}\left[v_{0}^{*}(k+1)-v_{0}(k+1)\right]
$$

where $v_{0}^{*}(k)$ and $v_{0}^{*}(k+1)$ are the reference output voltages at sampling instants $k$ and $k+1$ respectively. $v_{0}(k)$ is the measured voltage at sampling instant $k . v_{0}(k+1)$ is the predicted output voltage at sampling instant $k+1$.

Similarly in CCM, the cost function to be used is given as

$$
J=\left[i_{0}^{*}(k)-i_{0}(k)\right]^{T}\left[i_{0}^{*}(k)-i_{0}(k)\right]+\left[\left[i_{0}^{*}(k+1)-i_{0}(k+1)\right]\right]^{T}\left[i_{0}^{*}(k+1)-i_{0}(k+1)\right]
$$

where $i_{0}^{*}(k)$ and $i_{0}^{*}(k+1)$ are the reference output currents at sampling instants $k$ and $k+1$ respectively. $i_{0}(k)$ is the measured output current at sampling instant $k . i_{0}(k+1)$ is the predicted output current at sampling instant $k+1$.

In order to calculate and evaluate the cost function for different switching states of a threephase inverter operating in CCM, the value of the predicted output current $i_{0}(k+1)$ to be determined is explained in the next step. 
2) Prediction of current, calculation of cost function and selection of optimal switching state From the discrete state-space model given in (3.11), the value of the predicted current $i_{d}(k+1)$ is obtained as follows:

$$
i_{d}(k+1)=\left(1-\frac{R_{l} T_{s}}{L_{f}}\right) i_{d}(k)-\frac{T_{s}}{L_{f}} v_{0}(k)+\frac{T_{s}}{L_{f}} v_{s}
$$

Since $i_{0}(k+1) \approx i_{d}(k+1)$ at high sampling frequency, $i_{0}(k+1)$ is obtained as

$$
i_{0}(k+1) \approx i_{d}(k+1)=\left(1-\frac{R_{l} T_{s}}{L_{f}}\right) i_{d}(k)-\frac{T_{s}}{L_{f}} v_{0}(k)+\frac{T_{s}}{L_{f}} v_{s}
$$

All the terms in (3.22) are defined in Section 3.3. The three-phase switching voltage vector $v_{s}$ given in (3.7) is

$$
v_{s}=V_{d c} S
$$

where $S$ is the switching signal space vector which is defined as [36]:

$$
S=\frac{2}{3}\left(S_{a}+\alpha S_{b}+\alpha^{2} S_{c}\right) \text { where } \alpha=e^{j(2 \pi / 3)}
$$

The switching states $\left(S_{i 1}-S_{i 6}\right)$ of the power switches in the three-phase inverter model shown in Figure 3-3 have binary values " 1 " and " 0 " in a closed state and an open state respectively. These switching states of the power switches in the three-phase inverter model can be represented by the switching signals $S_{a}, S_{b}$ and $S_{c}$ as follows [36]:

$$
\begin{aligned}
& S_{a}=\left\{\begin{array}{l}
1, \text { if } S_{i 1} \text { on and } S_{i 2} \text { off } \\
0, \text { if } S_{i 1} \text { off and } S_{i 2} \text { on }
\end{array} ; S_{b}=\left\{\begin{array}{l}
1, \text { if } S_{i 3} \text { on and } S_{i 4} \text { off } \\
0, \text { if } S_{i 3} \text { off and } S_{i 4} \text { on }
\end{array} ;\right.\right. \\
& S_{c}=\left\{\begin{array}{l}
1, \text { if } S_{i 5} \text { on and } S_{i 6} \text { off } \\
0, \text { if } S_{i 5} \text { off and } S_{i 6} \text { on }
\end{array}\right.
\end{aligned}
$$

There are eight possible combinations of $S_{a}, S_{b}$ and $S_{c}$ and thus eight possible values of $v_{s}$, which are tabulated in Table 3-1 as shown. 
Table 3-1: Switching states and voltage vectors [36]

\begin{tabular}{cccc}
\hline$S_{a}$ & $S_{b}$ & $S_{c}$ & Voltage Vector $v_{s}$ \\
\hline 0 & 0 & 0 & $\frac{-1}{3} V_{d c}-j \frac{\sqrt{3}}{3} V_{d c}$ \\
\hline 0 & 0 & 1 & $\frac{-1}{3} V_{d c}+j \frac{\sqrt{3}}{3} V_{d c}$ \\
\hline 0 & 1 & 0 & $\frac{-2}{3} V_{d c}$ \\
\hline 1 & 0 & 0 & $\frac{2}{3} V_{d c}$ \\
\hline 1 & 0 & 1 & $\frac{1}{3} V_{d c}-j \frac{\sqrt{3}}{3} V_{d c}$ \\
\hline 1 & 1 & 0 & $\frac{1}{3} V_{d c}+j \frac{\sqrt{3}}{3} V_{d c}$ \\
\hline 1 & 1 & 1 & 0 \\
\hline & & & \\
\hline
\end{tabular}

Substituting the above tabulated values of $v_{s}$ in (3.22), eight different values of the predicted output current $i_{0}(k+1)$ are obtained. Then the cost function given in (3.20) is calculated for these eight different values of the predicted output current. Thus the eight different values of cost function are obtained. The voltage vector $v_{s}$ and the switching state which correspond to the minimum value of cost function are selected. This switching state is the optimal switching state and the corresponding switching signals are generated and applied to the three-phase inverter model to obtain a minimized cost function for the output current. Similar procedure can be followed for predicting the output voltage $v_{0}(k+1)$, calculating the cost function (3.19) for different switching states and selecting the switching state, corresponding to the minimum value of cost function. 


\subsection{Simulation Studies}

Simulation studies are conducted using MATLAB/Simulink to verify the performance of the MMG system. For simulation purpose, the number of microgrids in the MMG system is taken as 3 (i.e., $n=3$ ). Three test cases are conducted to demonstrate the effective control of the MMG system during various operating conditions. The first test case implements the effective exchange of sufficient power between the islanded microgrid and adjacent gridconnected microgrids in the MMG system. The second and third test cases demonstrate underfrequency load shedding in an islanded microgrid when the islanded microgrid receives insufficient power from the adjacent grid-connected microgrids. The parameters of the proposed MMG system are given in Table 3-2. The loss resistance of the inverter is coarsely estimated because it is not precisely known in practice. In the proposed system, the DG and battery units shown actually represent an aggregation of DG and battery units respectively. Assume that out of total load of $10 \mathrm{MW}$ in microgrid 2 in the MMG system, $7 \mathrm{MW}$ is nonsensitive load and remaining $3 \mathrm{MW}$ is sensitive load.

Table 3-2: Parameters of the proposed MMG system

\begin{tabular}{ll}
\hline Parameter & Value \\
\hline Inverter input voltage & $V_{d c}=800 \mathrm{~V}$ \\
\hline Loss resistance of inverter & $R_{l}=1 \mu \Omega$ \\
\hline LC filter of inverter & $L_{f}=1 \mu \mathrm{H}, C_{f}=0.02 \mathrm{~F}$ \\
\hline Supply voltage of distribution grid & $V_{g}=22000 / \sqrt{3} \mathrm{Vrms}$ (phase) \\
\hline Line resistance and inductance & $R_{t i}=1.6 \Omega, L_{t i}=6 \mathrm{mH}$ \\
& $(i=1,2,3)$ \\
\hline Leakage inductance of transformer & $L_{T}=21 \mu \mathrm{H}$ \\
\hline Details of different component ratings: & \\
\hline DG units 1, 2 and 3 & $4 \mathrm{MW}(\mathrm{max}), 4 \mathrm{MVAr}(\max )$ \\
\hline Storage battery units 1,2 and 3 & $3 \mathrm{MW} \mathrm{(max)}$ \\
\hline Transformers & $10 \mathrm{MVA}, 22 / 0.4 \mathrm{kV}$ \\
\hline Loads 1, 2 and 3 & $10 \mathrm{MW}, 4 \mathrm{MVAr}$ \\
\hline
\end{tabular}




\subsubsection{Test Case 1 - Islanded Microgrid Receiving Sufficient Power from Adjacent Grid-connected Microgrids}

Power flows in the MMG system during normal steady-state operation is shown in Figure 37. In the normal steady-state operation, all the microgrids in the MMG system are gridconnected and the DG units and the distribution grids meet the real and reactive power demands of the loads. Each distribution grid regulates the voltage of its respective microgrid during this operation. There is no power flow in the interconnecting lines and each distribution grid supplies $6 \mathrm{MW}$ to the load in its respective microgrid. The centralized controller always sends the desired real and reactive power reference signals to the LCs of the DG inverters in the microgrids during both grid-connected and islanded conditions. The DG inverter in each microgrid supplies $4 \mathrm{MW}$ and $4 \mathrm{MVAr}$ to the load. Battery inverter in each microgrid does not initially supply real and reactive power. Thus load demand is met in each microgrid in the MMG system during the steady-state operation.

The simulation begins with microgrid 2 operating in grid-connected mode for $0 \leq \mathrm{t}<4 \mathrm{~s}$. At $\mathrm{t}$ $=4 \mathrm{~s}$, microgrid 2 is islanded from distribution grid 2 due to a fault in the upstream network. When microgrid 2 is islanded from distribution grid 2, microgrid 2 will not be able to sustain its generation to meet the power demand of load 2. Thus, under this condition, microgrid 2 needs $2 \mathrm{MW}$ each from microgrid 1 and microgrid 3 in accordance with the contract agreement among the microgrids and takes $2 \mathrm{MW}$ locally from battery inverter 2 as shown in Figure 3-8. It is assumed that microgrids 1 and 3 have sufficient power to meet the load demand in microgrid 2. This power exchange is achieved with the use of the proposed control system explained in Section 3.3. To achieve this desired power exchange between the microgrids, the reference phase angle of load 2 bus voltage is calculated from (3.14). Then the centralized controller sends the voltage reference $230 \angle-0.85^{\circ}$ to the $\mathrm{LC}$ of battery inverter 2. Thus the phase angle of load 2 bus voltage is maintained at $-0.85^{\circ}$ for $\mathrm{t}>4 \mathrm{~s}$ as shown in Figure 3-9. It can be seen from Figure 3-10 that the magnitude of load 2 bus voltage is restored to $230 \mathrm{Vrms}$ for $\mathrm{t}>4 \mathrm{~s}$. Also, the transient disturbance in load 2 bus voltage lasts only for one-tenth of a cycle for $4 \leq \mathrm{t} \leq 4.002 \mathrm{~s}$. When the phase angle of load 2 bus voltage is changed, the real and reactive power flows through lines 1 and 2 are increased according to (3.1), (3.2) (3.3) and (3.4). In lines 1 and 2, the desired sending and receiving end real powers equal to 2.1 MW and 2 MW respectively are shown in Figure 3-11. Also, in lines 1 and 2, the reactive powers thus obtained at the sending and receiving ends equal to $1.74 \mathrm{MVAr}$ and 1.77 
MVAr respectively are shown in Figure 3-12. Battery inverters 1, 2 and 3 are tasked by the centralized controller to supply power at $\mathrm{t}=4.1 \mathrm{~s}, 0.1 \mathrm{~s}$ after islanding of microgrid 2 , as it takes a very short time of $0.1 \mathrm{~s}$ for these inverters to respond because of the signal transmission and processing in the centralized controller.

To supply the required real and reactive power through lines 1 and 2, the real power outputs of both battery inverters 1 and 3 are controlled to increase from $0 \mathrm{MW}$ to $2.1 \mathrm{MW}$ for $\mathrm{t}>4.1$ $\mathrm{s}$ as shown in Figure 3-13. The real power output of battery inverter 2 is maintained at $2 \mathrm{MW}$ for $\mathrm{t}>4.1 \mathrm{~s}$ as shown in Figure 3-13. As observed in Figure 3-14, the reactive power outputs of battery inverter 2 is increased from 0 MVAr to 3.54 MVAr for $\mathrm{t}>4.1 \mathrm{~s}$ and the reactive power outputs of battery inverters 1 and 3 are maintained at 0 MVAr. The battery inverter 2 supplies reactive power in order to meet the reactive power losses in the inductive reactances in lines 1 and 2. The magnitude and direction of real and reactive power flows in the MMG system during islanding of microgrid 2 are clearly shown in Figure 3-8.

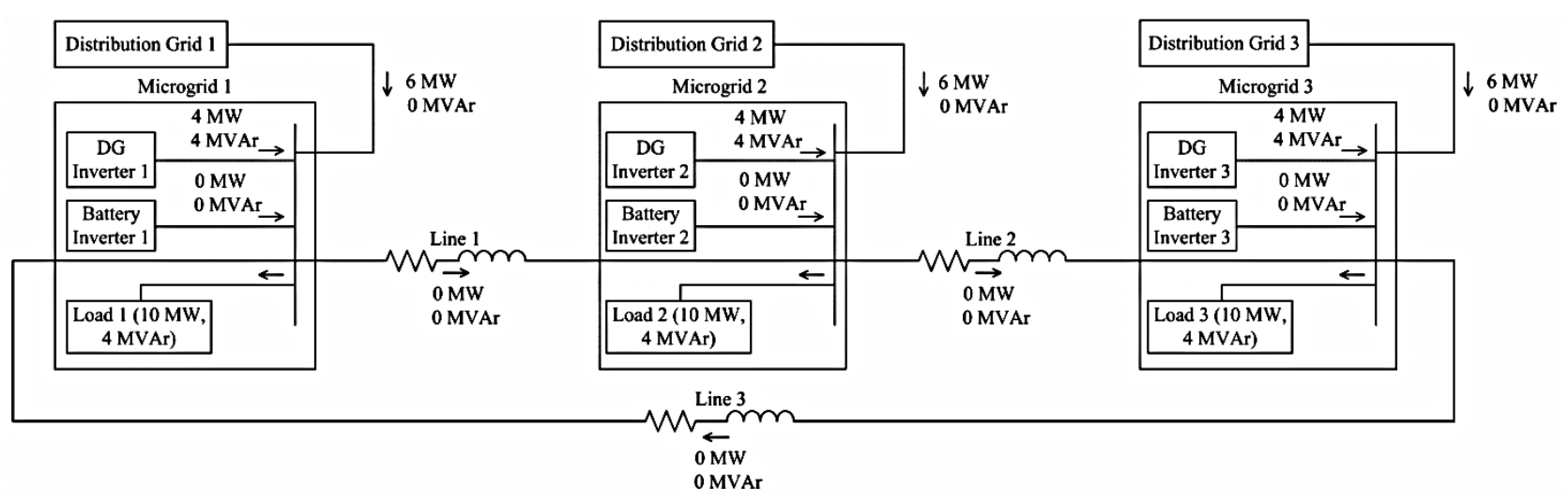

Figure 3-7: Power flows in the MMG system during steady-state operation.

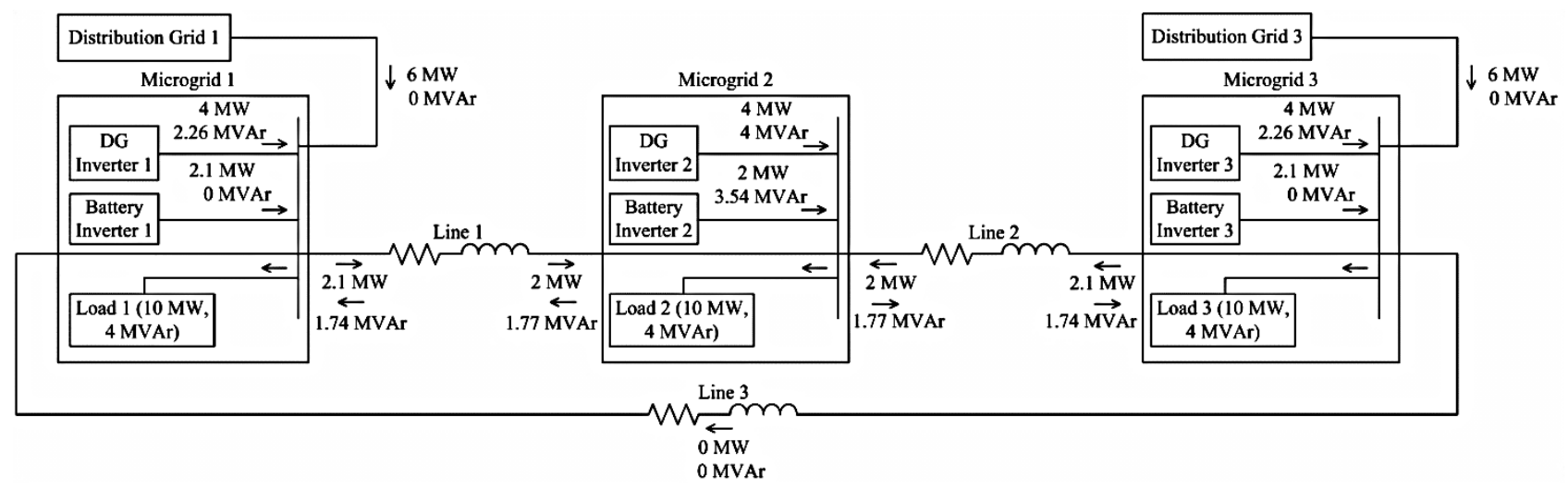

Figure 3-8: Power flows in the MMG system during islanding of microgrid 2 in test case 1. 
As shown in Figure 3-15, the real power outputs of DG inverters 1, 2 and 3 are maintained constant at $4 \mathrm{MW}$ for $0 \leq \mathrm{t} \leq 12 \mathrm{~s}$. In order to satisfy reactive power balance in microgrids 1 and 3 , the reactive power outputs of DG inverters 1 and 3 are decreased by the centralized and local controllers from 4 MVAr to 2.26 MVAr for $\mathrm{t}>4.1 \mathrm{~s}$. The reactive power output of DG inverter 2 is maintained at 4 MVAr itself for $0 \leq \mathrm{t} \leq 12 \mathrm{~s}$. The MPC controllers for these battery and DG inverters require only a period of about 10 cycles to track the real and reactive power reference signals of the order of MW and MVAr respectively during the initialization period. It can thus be observed that the proposed MPC controller is able to quickly track and settle to the power references.

With the loss of power supply from distribution grid 2, there is a power imbalance in microgrid 2 which causes the system frequency to drop to $49.9 \mathrm{~Hz}$ at $\mathrm{t}=4.1 \mathrm{~s}$ as shown in Figure 3-16. When this frequency drop is detected by the centralized controller, the battery inverters 1,2 and 3 are tasked to supply the required real power at $t=4.1 \mathrm{~s}$, as mentioned earlier, to meet the load demand in microgrid 2 and to restore the system frequency. However, due to system inertia, the system frequency continues to drop to $49.8 \mathrm{~Hz}$ at $\mathrm{t}=4.3 \mathrm{~s}$ and takes another $2.3 \mathrm{~s}$ to reach its nominal value. Thus, for $\mathrm{t} \geq 6.6 \mathrm{~s}$, the system frequency is gradually restored to its nominal value of $50 \mathrm{~Hz}$. Since load 2 bus voltage and system frequency are restored back to their nominal values after an islanding disturbance, voltage and frequency stability [103] can be guaranteed in the MMG system.

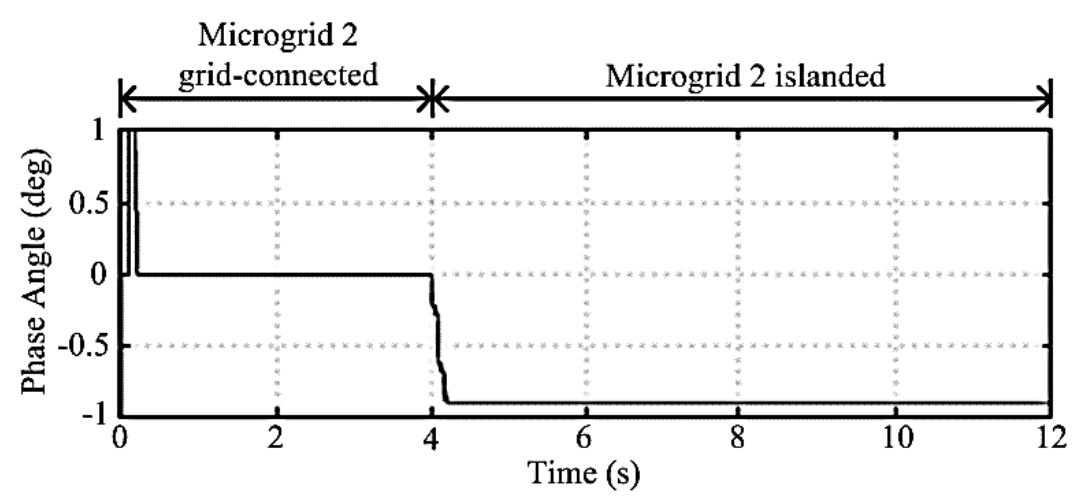

Figure 3-9: Phase angle of load 2 bus voltage. 


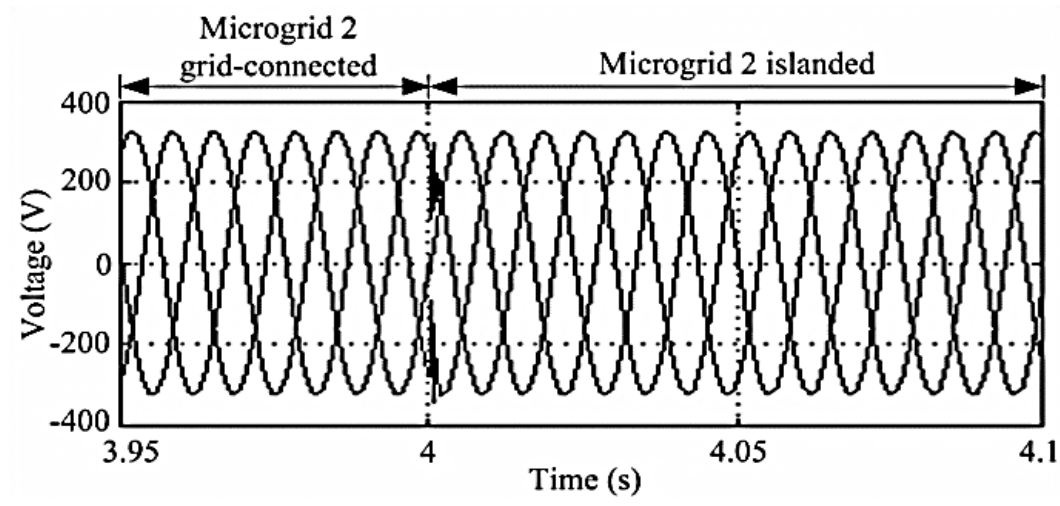

Figure 3-10: Three-phase load 2 bus voltage for $3.95 \leq \mathrm{t} \leq 4.1 \mathrm{~s}$.

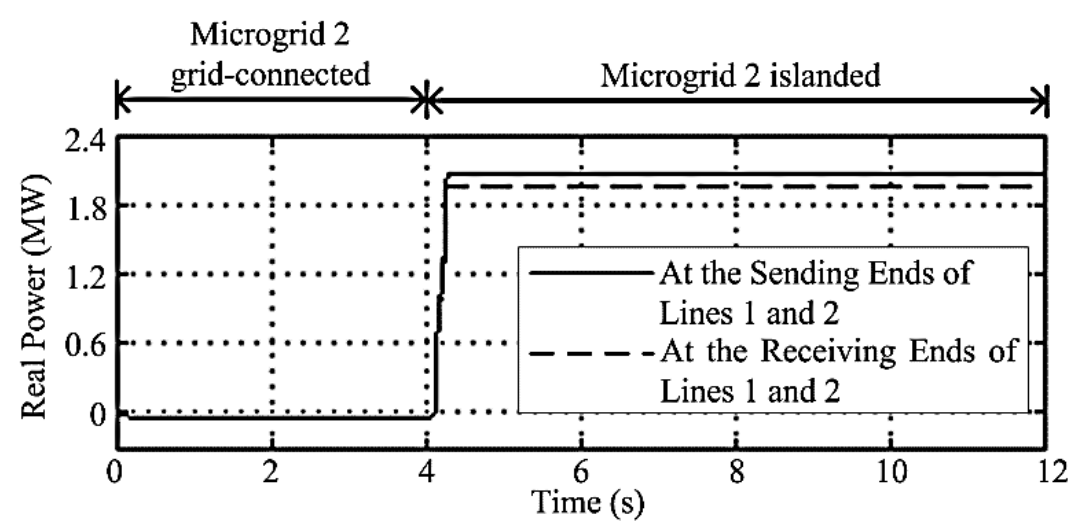

Figure 3-11: Real power at the sending and receiving ends of lines 1 and 2.

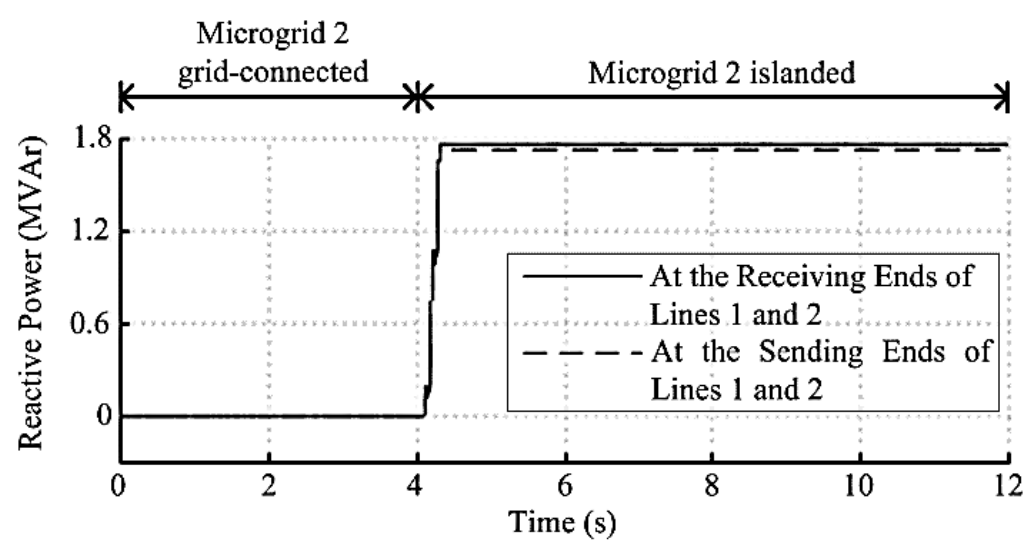

Figure 3-12: Reactive power at the sending and receiving ends of lines 1 and 2.

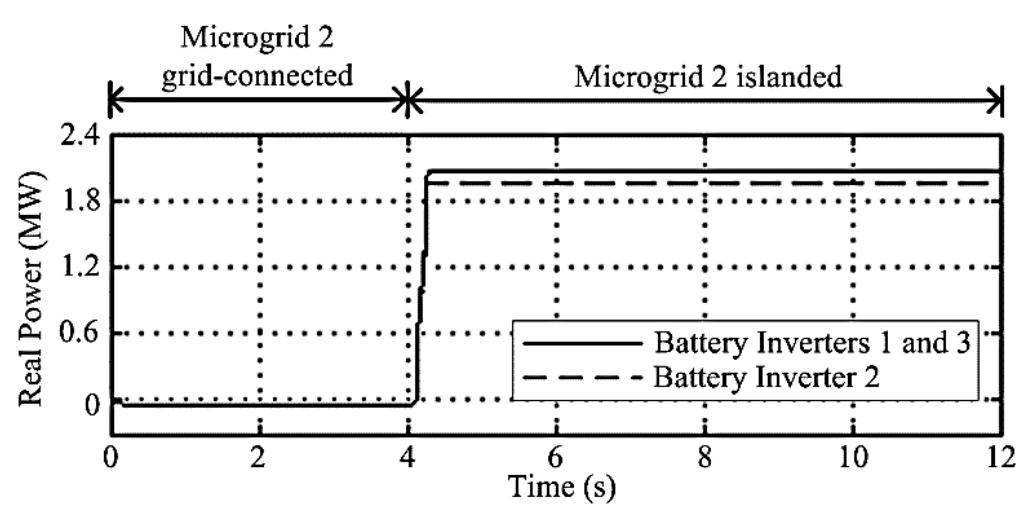

Figure 3-13: Real power outputs of battery inverters 1, 2 and 3. 


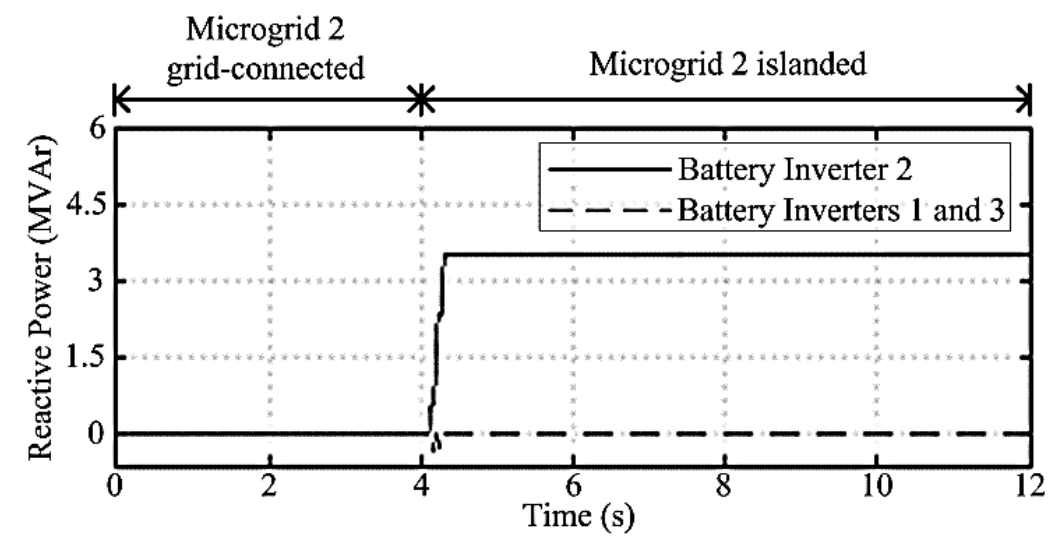

Figure 3-14: Reactive power outputs of battery inverters 1, 2 and 3.

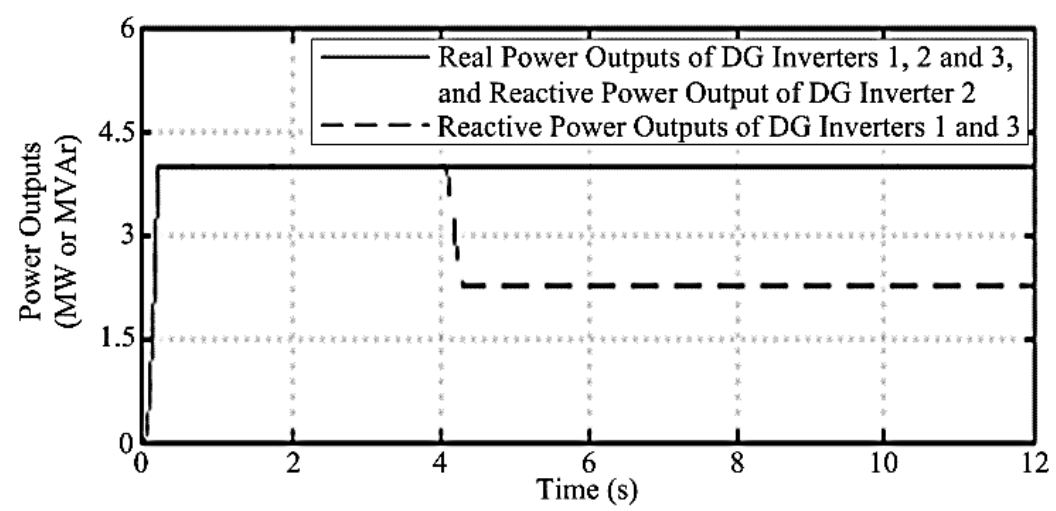

Figure 3-15: Real and reactive power outputs of DG inverters 1, 2 and 3.

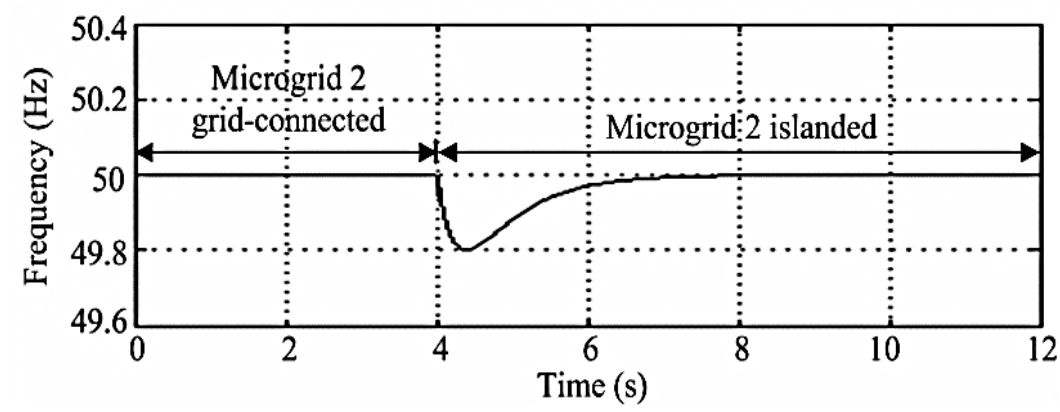

Figure 3-16: System frequency response.

\subsubsection{Test Case 2 - Islanded Microgrid Receiving Insufficient Power from Adjacent Grid-connected Microgrids}

In this test case, microgrids 1 and 3 do not have sufficient power to meet the load demand in islanded microgrid 2. At $\mathrm{t}=4 \mathrm{~s}$, microgrid 2 is islanded from distribution grid 2, resulting in a loss of $6 \mathrm{MW}$ in the same way as in test case 1 . After islanding, microgrid 2 requires $4 \mathrm{MW}$ remotely from microgrids 1 and 3 to meet its load demand. Due to low state of charge (SOC) of the storage battery units in microgrids 1 and 3, microgrid 2 is able to receive only $0.5 \mathrm{MW}$ 
each from microgrid 1 and microgrid 3 and thus battery inverter 2 in microgrid 2 supplies 3 MW locally as shown in Figure 3-17. The magnitude and direction of real and reactive power flows in the MMG system during islanding of microgrid 2 in test case 2 are clearly shown in Figure 3-17. In lines 1 and 2, the desired sending and receiving end real power flows are attained by sending the reference phase angle of load 2 bus voltage to the local controller of battery inverter 2 using the proposed control system. The phase angle of load 2 bus voltage is thus regulated at the reference value of $-0.29^{\circ}$ for $t>4 \mathrm{~s}$ as shown in Figure 3-18. At this reference value of phase angle, the sending and receiving end real power flows through lines 1 and 2 become equal to $0.51 \mathrm{MW}$ and $0.5 \mathrm{MW}$ respectively according to (3.1) and (3.2). To supply the sending end real power flows, the battery inverters 1 and 3 supply $0.51 \mathrm{MW}$ for $\mathrm{t}$ $>4.1 \mathrm{~s}$ as shown in Figure 3-19. It can also be seen that battery inverter 2 supplies $3 \mathrm{MW}$ for t $>4.1 \mathrm{~s}$. Since battery inverters 1 and 3 are unable to supply sufficient power to microgrid 2 , power imbalance occurs in microgrid 2, which results in drop of the system frequency beyond the tolerance limit.

The centralized controller detects the frequency drop and then implements the underfrequency load shedding scheme as proposed in Section 3.3. When the system frequency drops to $49.5 \mathrm{~Hz}$ at $\mathrm{t}=4.3 \mathrm{~s}$ as shown in Figure 3-20, 30\% of non-sensitive load (i.e., 2 MW) is shed in microgrid 2 as shown in Figure 3-21. The magnitude of load 2 bus voltage in this test case is maintained at $230 \mathrm{Vrms}$ by the proposed control system even if a transient disturbance, similar to that shown in Figure 3-10, occurs at the instant of load shedding. From Figure 3-20, it can be seen that after the first stage of load shedding, the system frequency is gradually restored to its nominal value of $50 \mathrm{~Hz}$ for $\mathrm{t} \geq 6.6 \mathrm{~s}$.

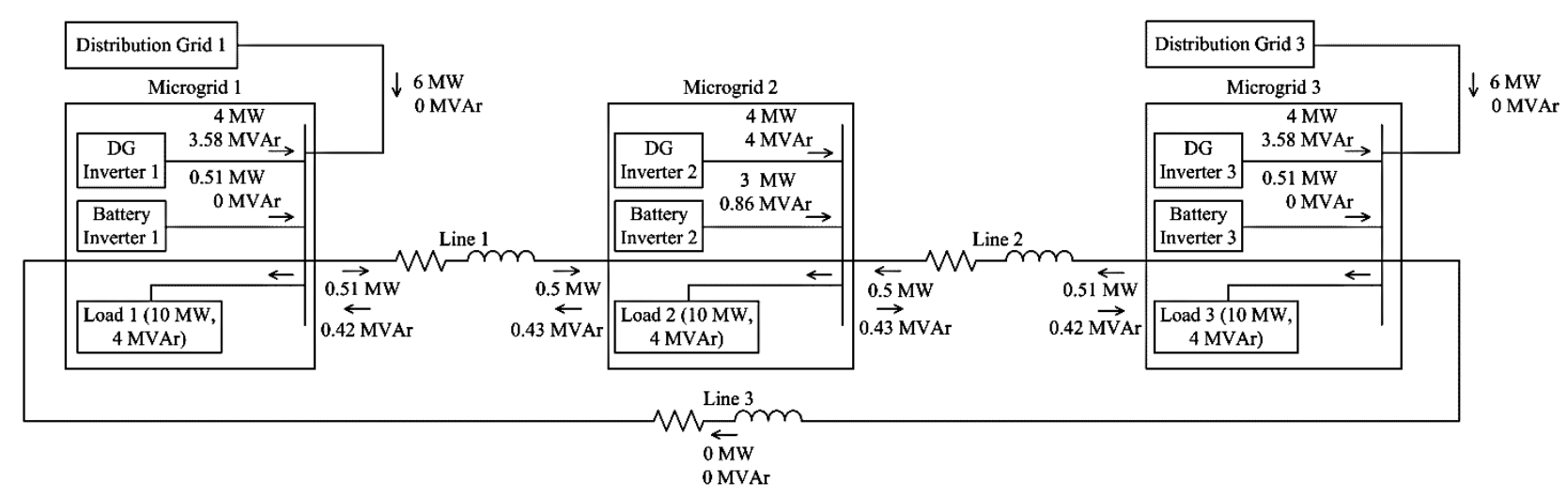

Figure 3-17: Power flows in the MMG system during islanding of microgrid 2 in test case 2. 


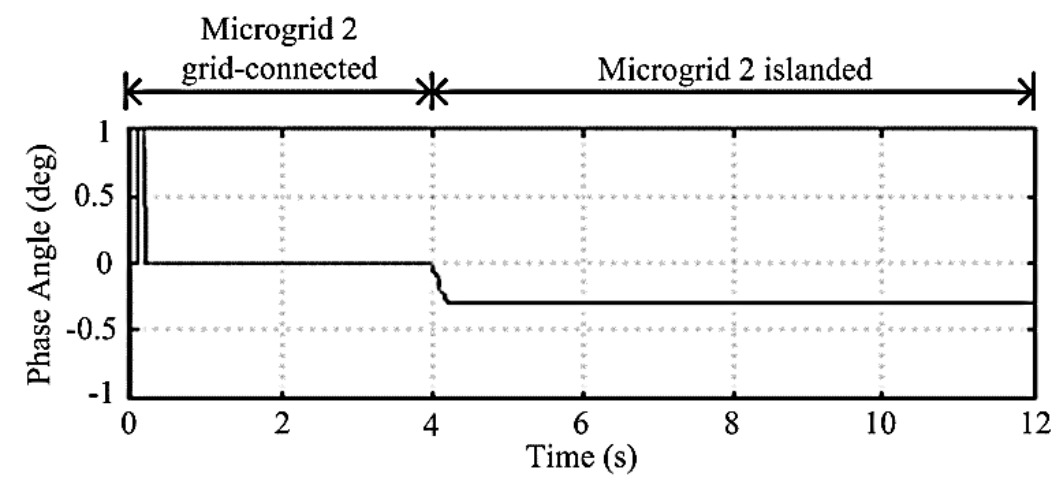

Figure 3-18: Phase angle of load 2 bus voltage.

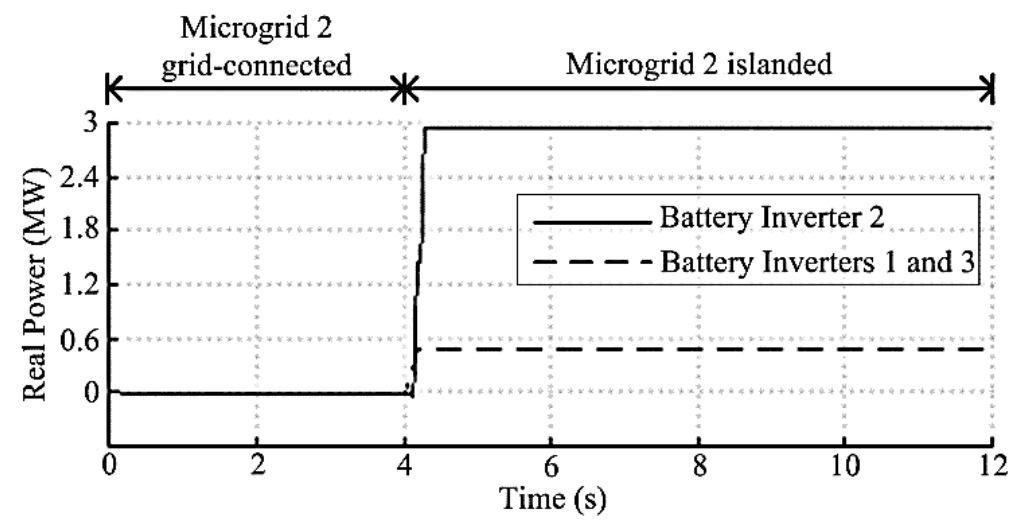

Figure 3-19: Real power outputs of battery inverters 1, 2 and 3.

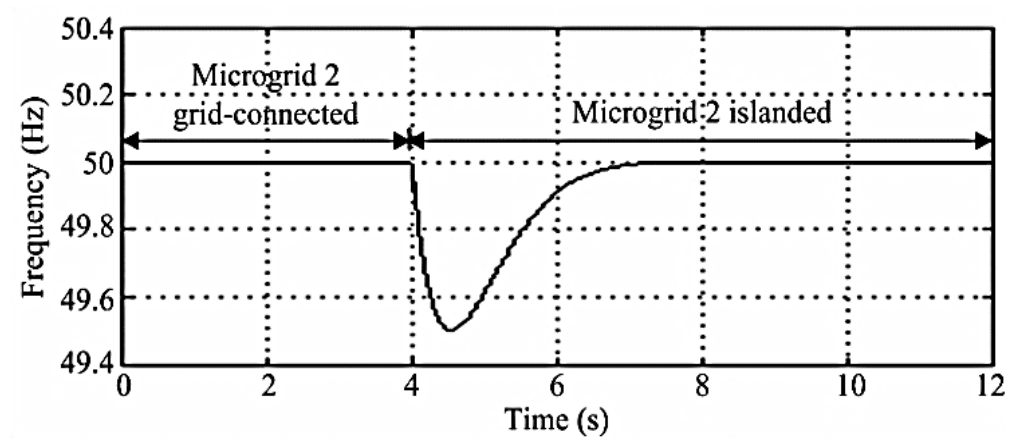

Figure 3-20: System frequency response.

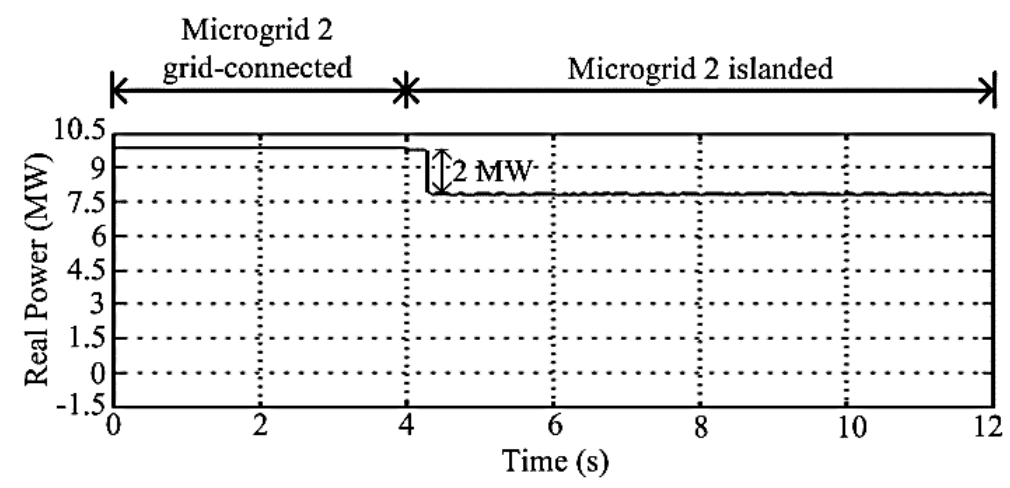

Figure 3-21: Real power consumed by load 2. 


\subsubsection{Test Case 3 - Failure of DG Inverter in Islanded Microgrid}

When microgrid 2 is islanded at $\mathrm{t}=4 \mathrm{~s}$, it results in a loss of $6 \mathrm{MW}$ from distribution grid 2 . At $t=4.05 \mathrm{~s}$, immediately after islanding of microgrid 2 as in test case 2, DG inverter 2 fails to operate in microgrid 2, as shown in Figure 3-22, resulting in an additional loss of $4 \mathrm{MW}$ of real power supplied to load 2. Battery inverter 2 still supplies $3 \mathrm{MW}$ to load 2 as in test case 2. Due to the failure of DG inverter in microgrid 2, the frequency deviation in microgrid 2 is beyond the tolerance limit of $\pm 0.5 \mathrm{~Hz}$, as shown in Figure 3-23. The zoom-in frequency response of microgrid 2 is shown in Figure 3-24. To restore the frequency of microgrid 2, the underfrequency load shedding is implemented by the centralized controller as follows:

- When the frequency of microgrid 2 drops to $49.5 \mathrm{~Hz}$ at $\mathrm{t}=4.1 \mathrm{~s}$ as shown in Figure 3-24, $30 \%$ of non-sensitive load (i.e., $2 \mathrm{MW}$ ) is shed in microgrid 2 as shown in Figure 3-25.

- When the frequency of microgrid 2 drops to $49 \mathrm{~Hz}$ at t $=4.2 \mathrm{~s}$ as shown in Figure 3-24, $35 \%$ of additional non-sensitive load (i.e., $2.5 \mathrm{MW}$ ) is shed in microgrid 2 as shown in Figure 3-25.

- When the frequency of microgrid 2 drops below $49 \mathrm{~Hz}$ at $\mathrm{t}=4.25 \mathrm{~s}$ as shown in Figure 324, lines 1 and 2 connected to microgrid 2 are tripped to isolate microgrid 2 from the rest of the system in order to restore the frequency of microgrids 1 and 3. After a time delay of $0.1 \mathrm{~s}$ (response time of centralized controller), the remaining non-sensitive load (i.e., 2.5 MW) is shed in microgrid 2 at $t=4.35 \mathrm{~s}$ as shown in Figure 3-25. As a result, the frequency of microgrid 2 is restored to its nominal value of $50 \mathrm{~Hz}$ for $\mathrm{t} \geq 7 \mathrm{~s}$ as observed in Figure 3-23. Different stages of load shedding are initiated in this test case within a very short time delay of $0.1 \mathrm{~s}$, which is the same as the time delay used in the load shedding scheme adopted in [108].

When the frequency of microgrid 2 drops below $49 \mathrm{~Hz}$, lines 1 and 2 are intentionally tripped at $\mathrm{t}=4.25 \mathrm{~s}$. To supply microgrid 2 with $0.5 \mathrm{MW}$ each from microgrids 1 and 3 from the instant of islanding until the lines 1 and 2 are tripped, the phase angle of load 2 bus voltage is thus regulated at the reference value of $-0.29^{\circ}$ for $4 \leq \mathrm{t} \leq 4.25 \mathrm{~s}$ and at the reference value of $0^{\circ}$ for $\mathrm{t}>4.25 \mathrm{~s}$ by the proposed control system as shown in Figure 3-26. As a result, the real power flows at the sending and receiving ends of lines 1 and 2 become equal to $0.51 \mathrm{MW}$ and $0.5 \mathrm{MW}$ respectively for $4 \leq \mathrm{t} \leq 4.25 \mathrm{~s}$ as observed in Figure 3-27. To supply these sending end real power flows, battery inverters 1 and 3 supply $0.51 \mathrm{MW}$ only for $4 \leq \mathrm{t} \leq 4.25 \mathrm{~s}$ until lines 1 and 2 are tripped as shown in Figure 3-28. 


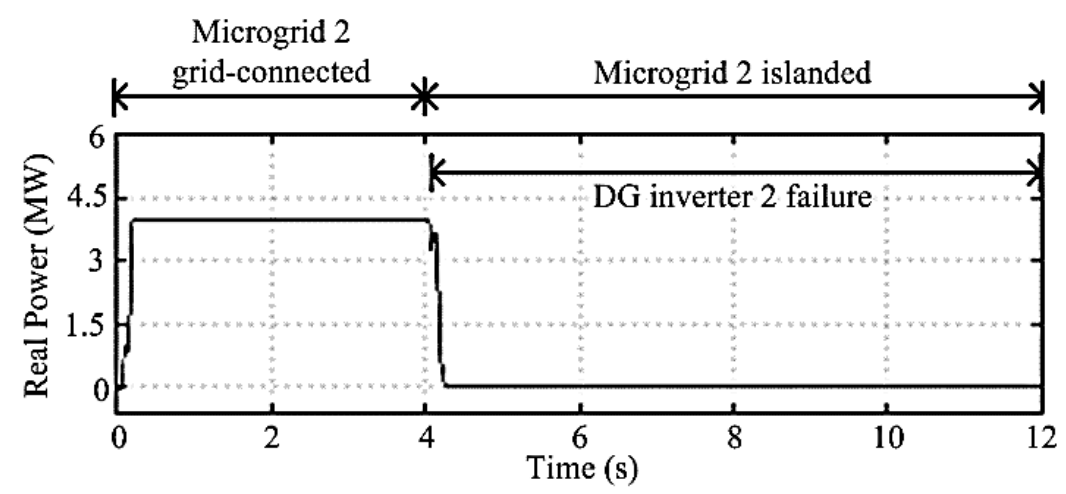

Figure 3-22: Real power output of DG inverter 2.

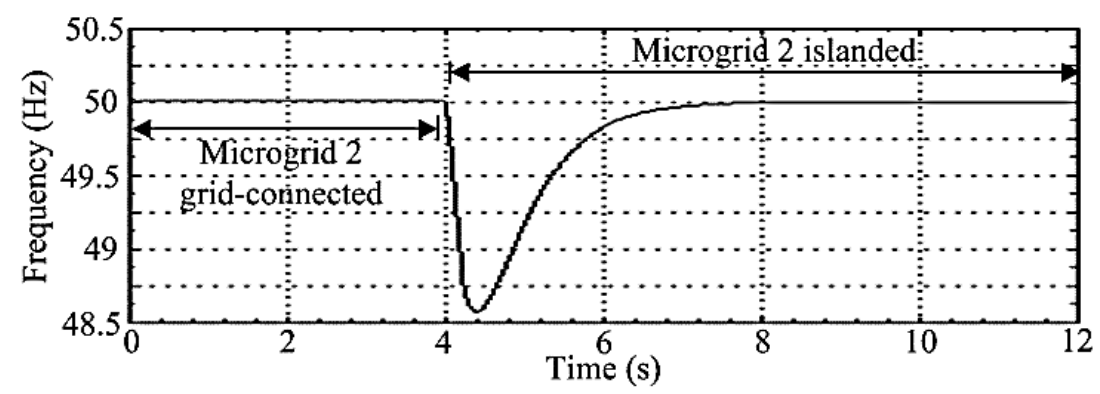

Figure 3-23: Frequency response of microgrid 2.

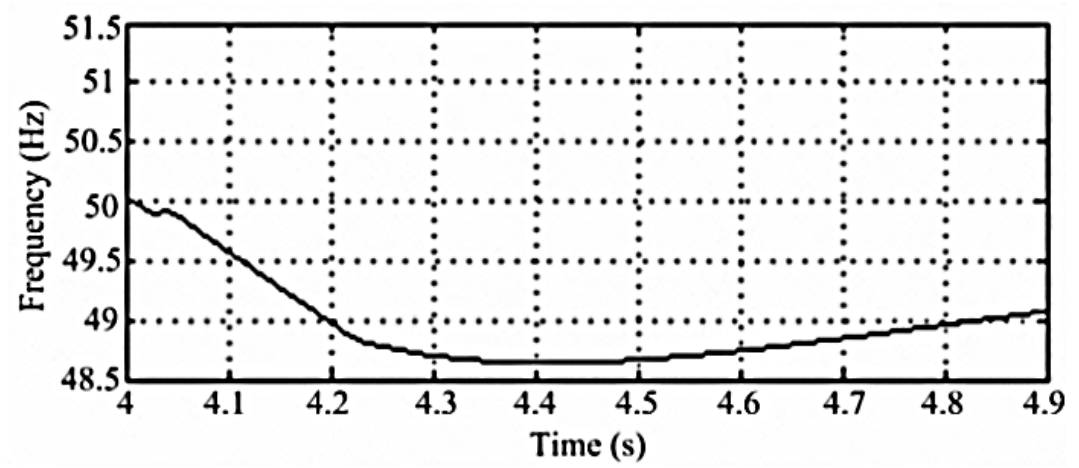

Figure 3-24: Zoom-in frequency response of microgrid 2 for $4 \leq \mathrm{t} \leq 4.9 \mathrm{~s}$.

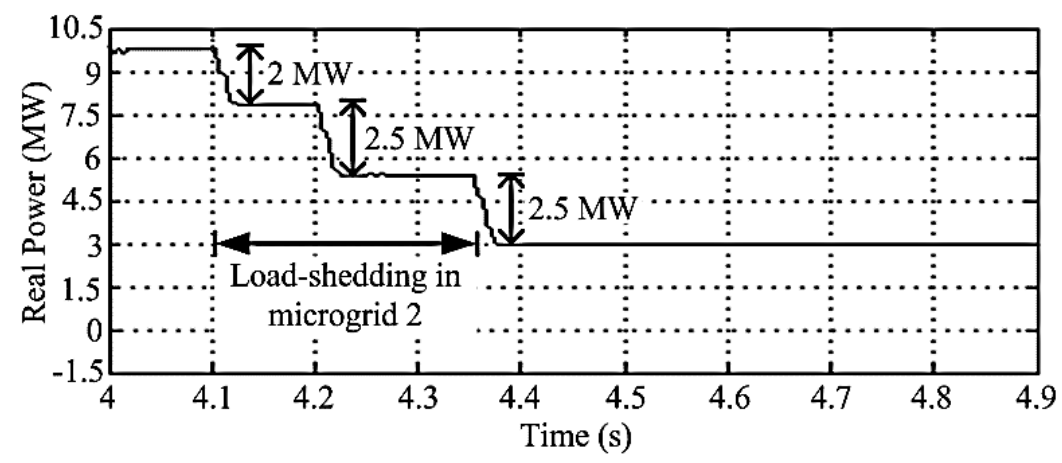

Figure 3-25: Real power consumed by load 2 for $4 \leq t \leq 4.9 \mathrm{~s}$. 


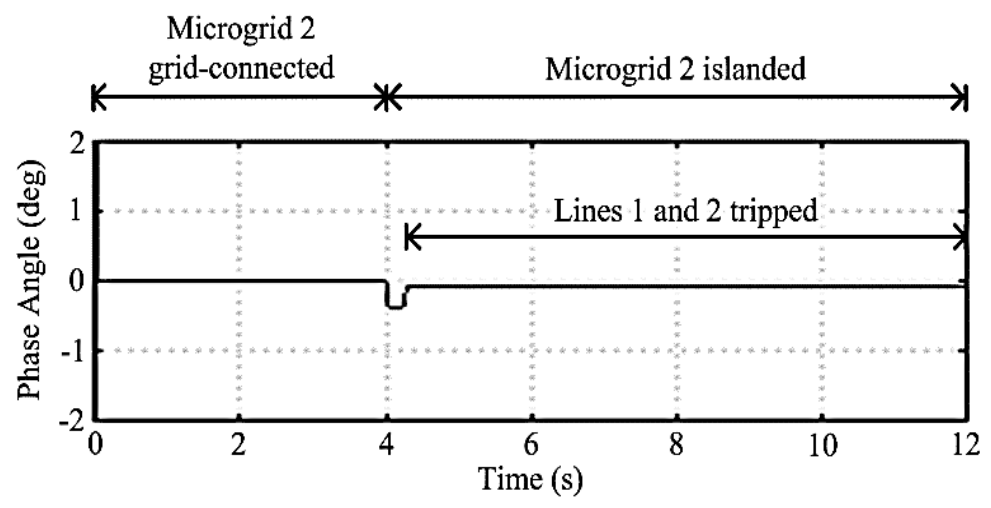

Figure 3-26: Phase angle of load 2 bus voltage.

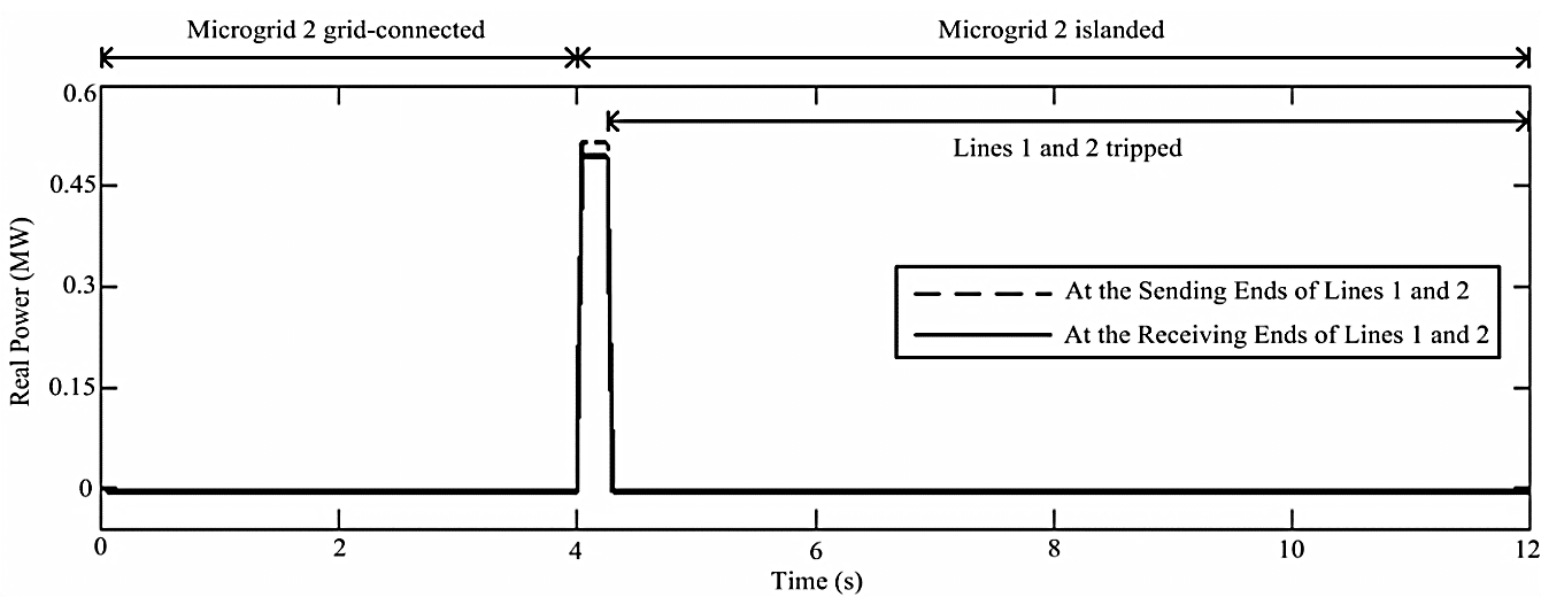

Figure 3-27: Real power flows at the sending and receiving ends of lines 1 and 2.

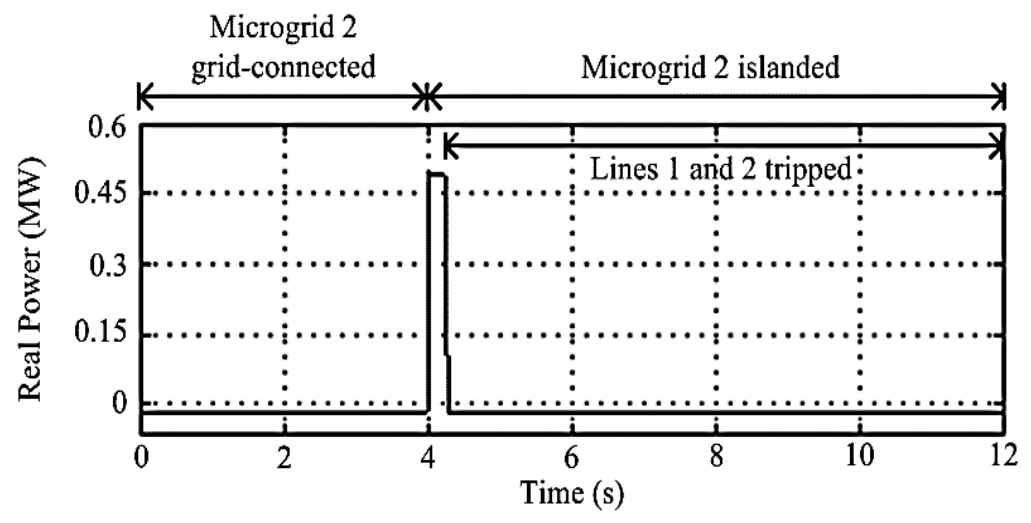

Figure 3-28: Real power outputs of battery inverters 1 and 3.

\subsection{Comparison of MPC and PI Controllers for an Inverter in the MMG System}

In the proposed AC/AC multi-area MMG system, the output currents of the inverters are controlled in order to regulate the output real and reactive powers of the inverters. In such a current-controlled MMG system, the different parameters to be considered to assess the 
dynamic performance of different control methods such as MPC and PI control are as follows [32]:

a) Settling time

b) Tracking error

c) Overshoot

Thus, based on the above parameters, the MPC controller proposed in Chapter 3 is compared with classical PI controller.

The rationale for choice of PI controller is explained as follows:

The use of power converters has become very popular in the last few decades for a wide range of applications, including drives, energy conversion, traction, and distributed generation [36]. The control of power converters has been extensively studied and new control schemes are presented every year [36]. Several control schemes have been proposed for the control of power converters and drives [36]. Among these control schemes, the most widely used and the most established control scheme in literature is PI control with pulse width modulation (PWM) [36]. Considering a modulator stage for the generation of control signals for the power switches of the converter allows the power electronic designer to easily linearize the nonlinear power converter. In this way, any linear controller can be used, the best and the most common choice is the PI controller [36]. Thus, based on these reasons, the PI controller is chosen and compared with the proposed MPC controller in Chapter 3.

One commonly adopted controller for the control of the inverter, which has been implemented in many research works [5], [54], is the PI controller. Simulation studies of the proposed MMG system shown in Figure 3-1 are conducted first using PI controller which is similar to that presented in [5]. The PI controller consists of inner voltage and current loops and an external power loop to regulate the output voltage and output power of the inverter respectively, which is discussed in detail in [5]. Simulation studies of the proposed MMG system are then performed using MPC controller. The parameters of the MMG system remain the same in both the simulation studies. The real power output of DG inverter 1 in microgrid 1 in the MMG system is maintained at 3 MW using PI and MPC controllers as shown in Figure 3-29 and Figure 3-30 respectively. Also, the reactive power output of DG inverter 1 is maintained at 3 MVAr using PI and MPC controllers as shown in Figure 3-31 and Figure 332 respectively. The performance of the PI and MPC controllers is compared in Table 3-3. From the various parameter values in Table 3-3, it can be inferred that MPC yields better performance when applied for the control of the inverter. Thus MPC is chosen as the preferred control method for the control of the inverters in the proposed MMG system. 


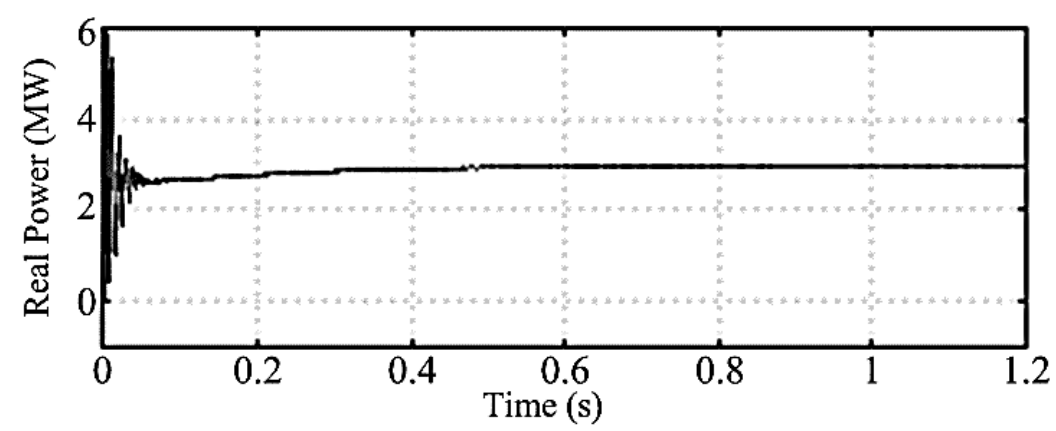

Figure 3-29: Real power output of DG inverter 1 with PI controller.

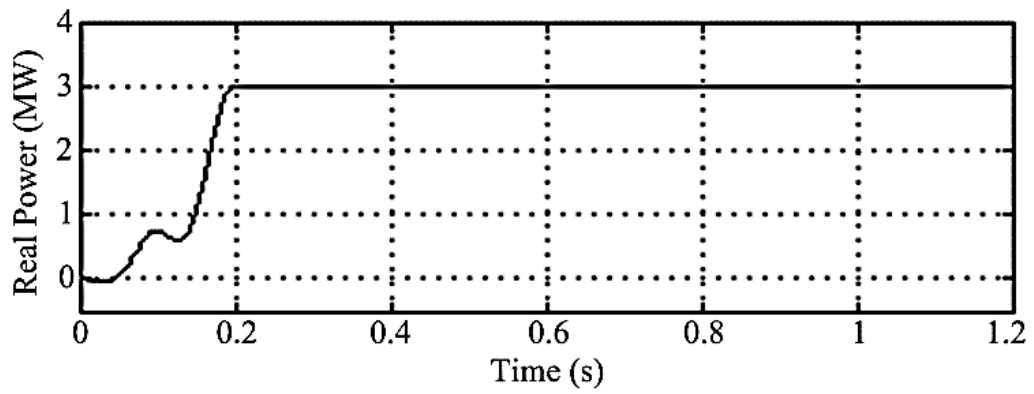

Figure 3-30: Real power output of DG inverter 1 with MPC controller.

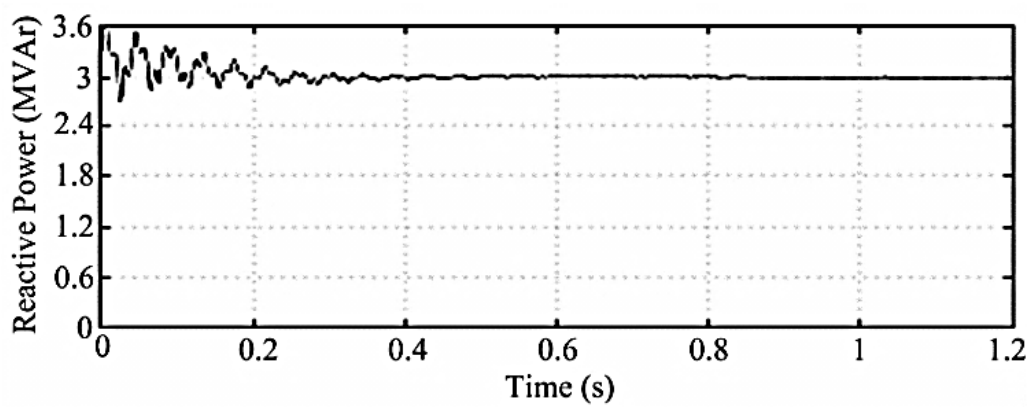

Figure 3-31: Reactive power output of DG inverter 1 with PI controller.

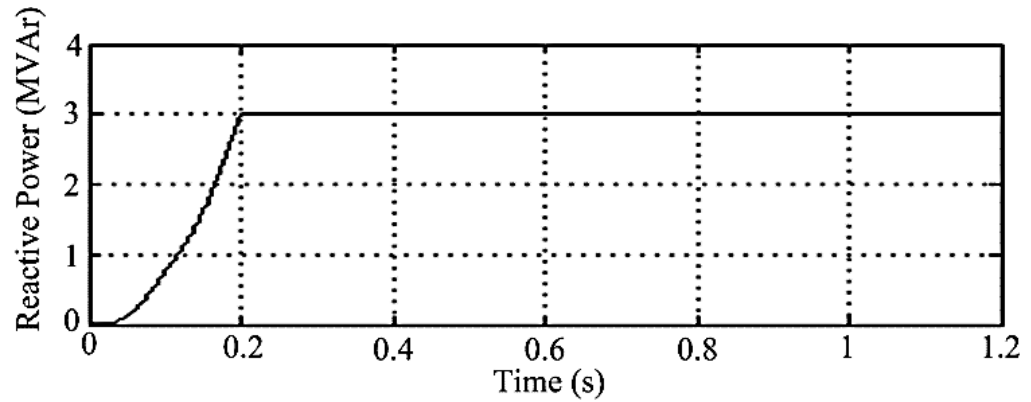

Figure 3-32: Reactive power output of DG inverter 1 with MPC controller.

Table 3-3: Performance of MPC and PI controllers

\begin{tabular}{l|l|l}
\hline Parameter & MPC controller & PI controller \\
\hline Settling time & 10 cycles & 25 cycles \\
\hline Tracking error & $\pm 0.2 \%$ & $\pm 2 \%$ \\
\hline Overshoot & $\pm 0.01 \%$ & $\pm 15 \%$ \\
\hline
\end{tabular}




\subsubsection{Comparison of MPC and PI Controllers for Test Case 2}

To compare the performance of PI and MPC controllers in the proposed MMG system, test case 2 is taken as the sample test case. In the proposed control system of the MMG system, local MPC controllers are used first and then local PI controllers are used for comparing the performance of both these controllers. PI controller used here is similar to that presented in [5]. As explained in test case 2, microgrid 2 is islanded from distribution grid 2 and is supplied with $0.5 \mathrm{MW}$ each from microgrids 1 and 3. To attain the desired sending and receiving end real power flows in lines 1 and 2, the phase angle of load 2 bus voltage is regulated at the reference value almost equal to the desired value of $-0.29^{\circ}$ for $\mathrm{t}>4 \mathrm{~s}$ using the local MPC controller of battery inverter 2 as shown in Figure 3-33. When the local PI controller of battery inverter 2 is used, the reference phase angle obtained for $\mathrm{t}>4 \mathrm{~s}$ is $-0.4^{\circ}$ as observed in Figure 3-33. Thus, there is a large tracking error of $33.33 \%$ for reference phase angle in case of PI controller while there is only a negligible tracking error of $0.1 \%$ for reference phase angle in case of MPC controller . At the reference phase angle of $-0.29^{\circ}$, the sending and receiving end real power flows through lines 1 and 2 become equal to $0.51 \mathrm{MW}$ and 0.5 MW respectively according to (3.1) and (3.2).

With MPC controller, battery inverters 1 and 3 are capable of supplying real power almost equal to 0.51 MW for $\mathrm{t}>4.1 \mathrm{~s}$ as shown in Figure 3-34. In case of PI controller, battery inverters 1 and 3 supply 0.52 MW for $\mathrm{t}>4.1 \mathrm{~s}$ as observed in Figure 3-34. Similarly, battery inverter 2 with MPC controller supplies the desired power almost equal to $3 \mathrm{MW}$ for $\mathrm{t}>4.1$ $\mathrm{s}$, whereas battery inverter 2 with PI controller supplies 3.07 MW for $\mathrm{t}>4.1 \mathrm{~s}$ as shown in Figure 3-35. The delay time taken for the battery inverters 1, 2 and 3 with MPC controllers to reach their power set-points after $\mathrm{t}=4.1 \mathrm{~s}$ is only 10 cycles, whereas the delay time taken for the battery inverters 1, 2 and 3 with PI controllers to reach their power set-points after $t=4.1$ $\mathrm{s}$ is around 25 cycles. The performance of MPC and PI controllers in power reference tracking for test case 2 is given in Table 3-4. From Table 3-4, it can be inferred that MPC controller yields better power tracking performance than PI controller in terms of smaller tracking error and shorter delay time when applied for the control of inverters in the MMG system. With both MPC and PI controllers, the system frequency drops to the same value of $49.5 \mathrm{~Hz}$ at $\mathrm{t}=4.3 \mathrm{~s}$ during islanding of microgrid 2 as shown in Figure 3-36. But with MPC controller, the system frequency is quickly restored to its nominal value in $2.3 \mathrm{~s}$ after microgrid islanding, whereas with PI controller, the system frequency restoration takes 
around $4 \mathrm{~s}$ after microgrid islanding. Since the system frequency drop is the same for MPC and PI controllers, load shedding is implemented in test case 2 for both MPC and PI controllers at $t=4.3 \mathrm{~s}$. Thus, in test case 2 , the simulation result showing the real power consumed by load 2 in Figure 3-21 remains unchanged for both MPC and PI controllers.

Table 3-4: Performance of MPC and PI controllers in power reference tracking for test case 2

\begin{tabular}{lll}
\hline Parameter & MPC controller & PI controller \\
\hline Tracking error & $\pm 0.2 \%$ & $\pm 2 \%$ \\
\hline Delay time for power set-point change & 10 cycles & 25 cycles \\
\hline
\end{tabular}

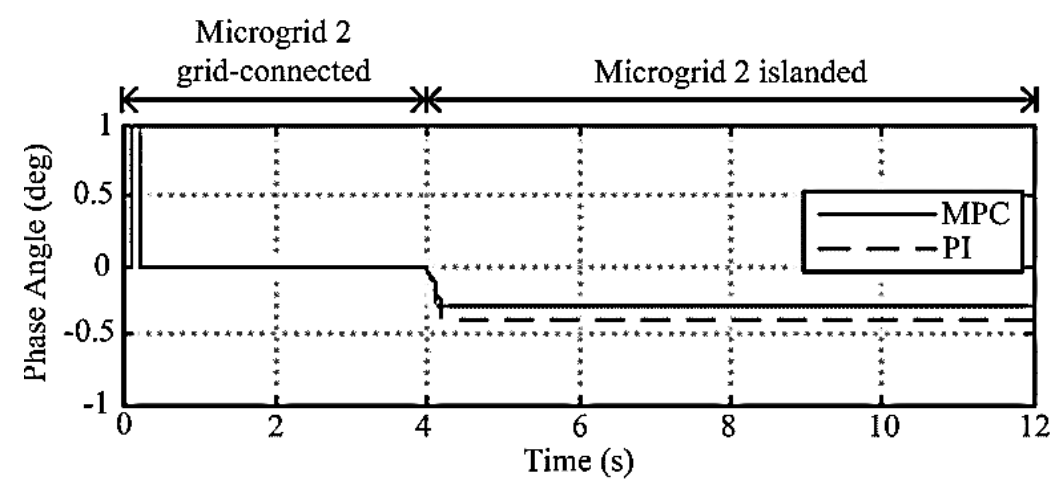

Figure 3-33: Phase angle of load 2 bus voltage.

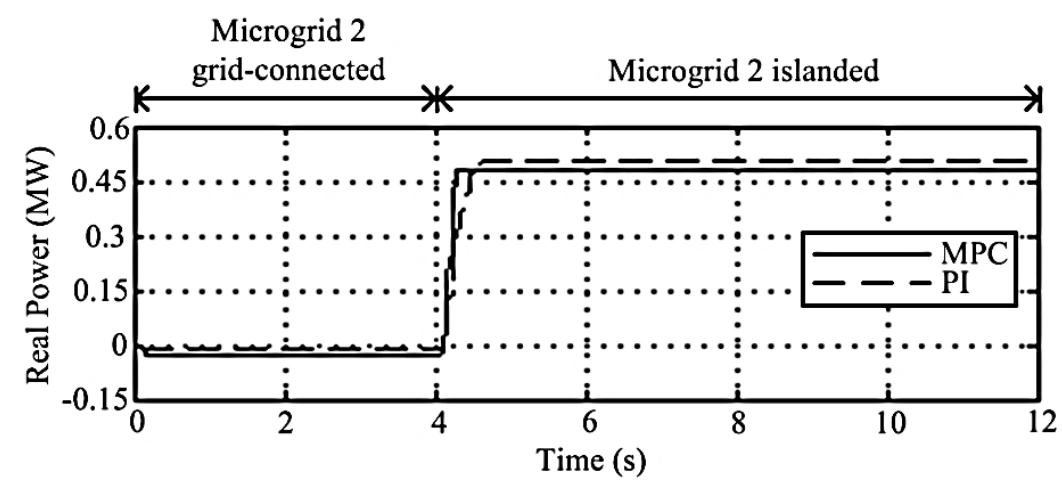

Figure 3-34: Real power outputs of battery inverters 1 and 3.

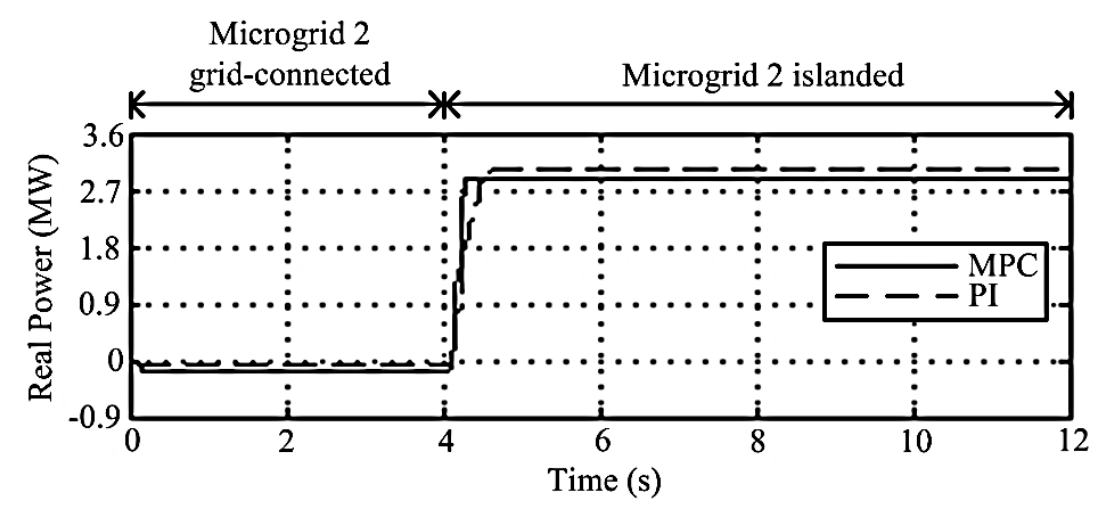

Figure 3-35: Real power output of battery inverter 2. 


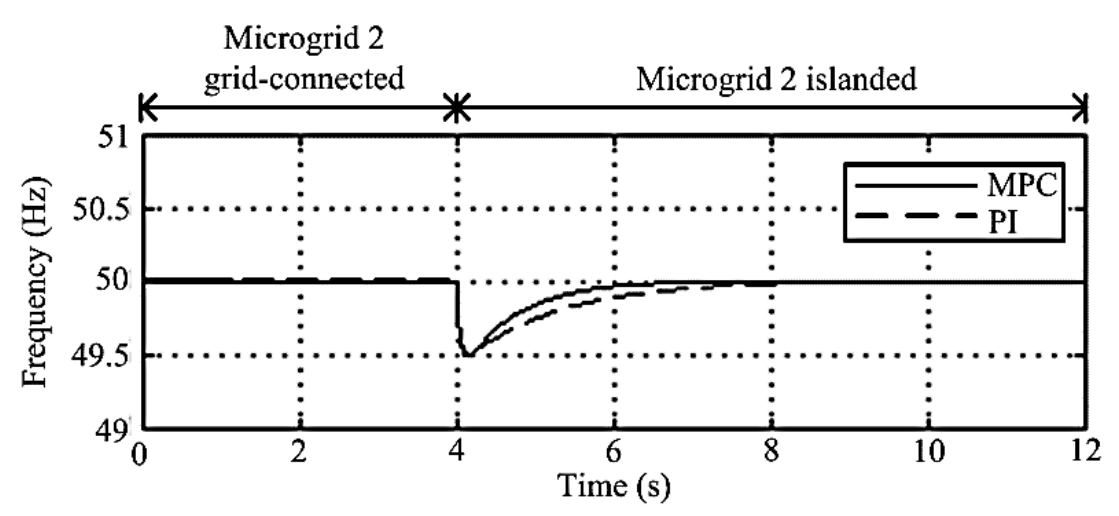

Figure 3-36: System frequency response.

\subsection{Conclusion}

In this chapter, a control system for an AC/AC multi-area MMG system has been presented to solve the voltage and frequency variation problems during islanding of a microgrid. An improved MPC algorithm has been developed for the control of DG and battery inverters in the proposed MMG system. Simulation studies have been conducted to validate the effectiveness of the proposed control system. The simulation results have shown that the developed control system can achieve good performance and the MMG system is able to operate efficiently and accurately during islanding and load shedding. The developed control system in the MMG system can facilitate desired power exchange among different microgrids under various operating conditions and can also achieve effective voltage and frequency regulation during microgrid islanding. Thus an effectively controlled multi-area MMG system can fulfil basic objectives such as improved reliability of supply, enhanced voltage and frequency stability and effective dynamic islanding. The proposed design concept for the MMG system serves as a fundamental step for the hardware implementation of the MMG system, which will have a scope for further research in the area of multi-microgrids. To further extend the study of multi-area MMG systems, another configuration of an AC/DC multi-area MMG system, consisting of both AC and DC microgrids, is proposed in the next chapter. 


\section{CHAPTER 4 VOLTAGE AND POWER CONTROL IN AN AC/DC MULTI-AREA MMG SYSTEM}

\subsection{Introduction}

This chapter presents an overall configuration for an AC/DC multi-area MMG system, consisting of interconnected $\mathrm{AC}$ and $\mathrm{DC}$ microgrids. In addition, a control method is proposed to regulate DC load bus voltage during DC microgrid islanding in an AC/DC multiarea MMG system. In the event of islanding of a DC microgrid from the distribution grid in the proposed MMG system, real power shortage occurs in the DC microgrid and DC load bus voltage of the islanded DC microgrid is affected. To overcome these problems, a control system for the AC/DC MMG system is proposed. The proposed control system facilitates desired power exchange between grid-connected AC microgrids and islanded DC microgrid, and achieves effective voltage regulation in the MMG system. The main significance of the proposed MMG system is that multiple AC and DC microgrids in different locations can be interconnected to meet larger bulk power demands. This ensures improved reliability and security of power supply in the MMG system. A state-space model based MPC algorithm is used to regulate various parameters such as output voltage and power of the converters and inverters in the MMG system. The performance of the proposed MMG system is verified during islanding, load increase and load shedding using simulation studies. The simulation studies show that the converters and inverters can operate effectively using MPC to provide the desired voltage and power during islanding, load increase and load shedding.

\subsection{System Description}

The overall configuration of the proposed AC/DC multi-area MMG system is shown in Figure 4-1. It consists of three microgrids, each of them separated from the others by interconnecting lines of about $10 \mathrm{~km}$. Microgrids 1 and 3 are $\mathrm{AC}$ microgrids and microgrid 2 is a DC microgrid. The DC microgrid is interconnected with the AC microgrids through interconnecting inverters 1 and 2, and lines 1 and 2. It is assumed that the main distribution grid is sectionalized into distribution grid 1, distribution grid 2 and distribution grid 3. Each microgrid is connected to its respective distribution grid through a $0.4 / 22 \mathrm{kV}$ distribution transformer. The load bus voltages are represented by $V_{L 1} \angle \delta_{1}, V_{L 2}$ and $V_{L 3} \angle \delta_{3}$. The output voltages of the interconnecting inverters are denoted by $V_{n 1} \angle \delta_{n 1}$ and $V_{n 2} \angle \delta_{n 2}$. Voltages at the 
end of the lines are represented by $V_{1} \angle \delta_{1}, V_{2} \angle \delta_{2}$ and $V_{3} \angle \delta_{3}$. Line resistances and inductances are represented as $R_{t i}(i=1,2$ and 3$)$ and $L_{t i}(i=1,2$ and 3$)$ respectively. The sending end real and reactive power flows through the lines are represented as $P_{s i}(i=1,2$ and 3$)$ and $Q_{s i}(i=1$, 2 and 3). The receiving end real and reactive power flows through the lines are represented as $P_{r i}(i=1,2$ and 3$)$ and $Q_{r i}(i=1,2$ and 3$)$.

Each microgrid in the proposed MMG system basically operates in two different modes of operation: (1) grid-connected mode when the microgrid is connected to its respective distribution grid; and (2) islanded mode when the microgrid is disconnected from its respective distribution grid. The schematic diagram of an AC microgrid used in the proposed MMG system is shown in Figure 4-2 (a). The AC microgrid consists of a DG unit, a storage battery unit and an AC load. The DG unit is connected to the load bus through a DC/DC boost converter and a DG DC/AC inverter. The storage battery unit is connected to the load bus through a bi-directional DC/DC converter and a battery DC/AC inverter. The real and reactive power outputs of DG inverter $i$ are represented as $P_{d i}$ and $Q_{d i}$ respectively. The real and reactive power outputs of battery inverter $i$ are represented as $P_{b i}$ and $Q_{b i}$ respectively. The schematic diagram of DC microgrid used in the proposed MMG system is shown in Figure 4-2 (b). The DC microgrid consists of a DG unit, a storage battery unit and a supercapacitor unit. The DG, storage battery and supercapactor units are connected to the DC load bus through their DC/DC buck converters $2 \mathrm{a}, 2 \mathrm{~b}$ and $2 \mathrm{c}$ respectively. The real power outputs of the DC/DC buck converters $2 \mathrm{a}, 2 \mathrm{~b}$ and $2 \mathrm{c}$ are represented by $P_{d 2}, P_{b 2}$ and $P_{s 2}$ respectively. The inverters and converters in the proposed MMG system can operate in two different modes, the first mode called CCM where output currents of these inverters and converters are regulated at the desired values and the second mode called VCM where output voltages of these inverters and converters are regulated at the desired values.

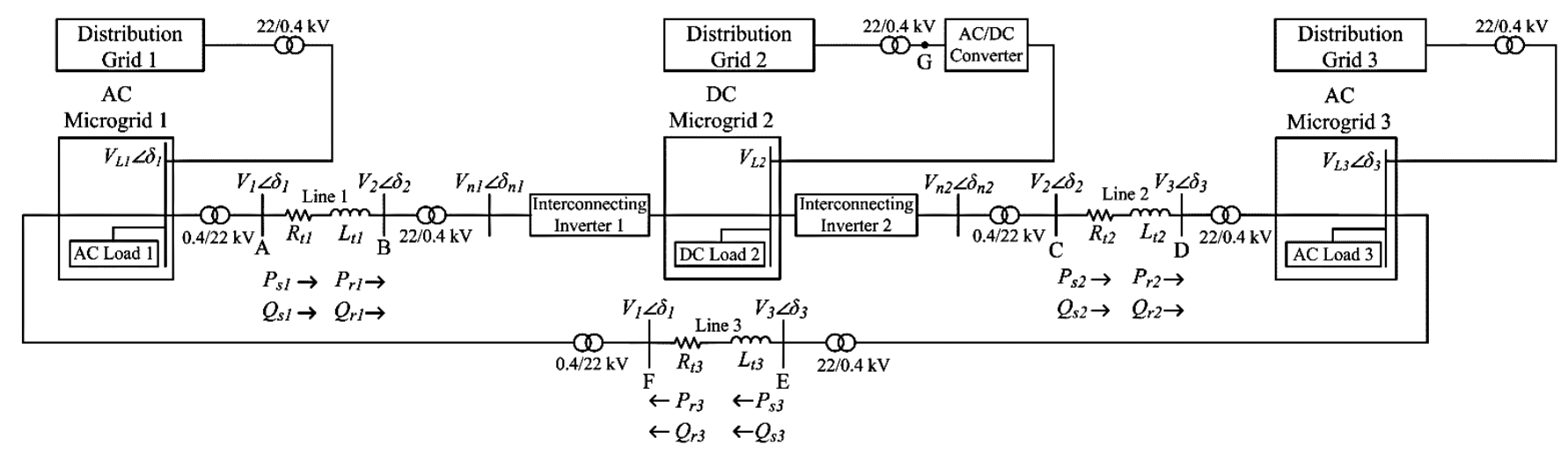

Figure 4-1: Overall Configuration of the proposed AC/DC multi-area MMG system. 


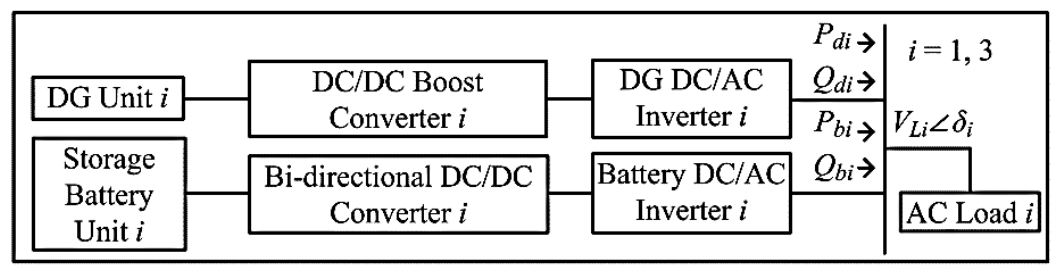

(a)

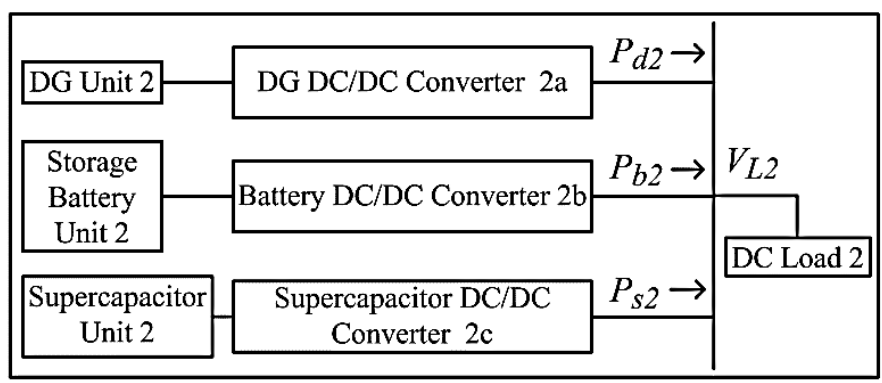

(b)

Figure 4-2: Schematic diagram of microgrids: (a) AC microgrids 1 and 3, (b) DC microgrid 2.

During grid-connected mode of the AC microgrid, the distribution grid regulates the load bus voltage and frequency, and thus both DG and battery inverters in the microgrid operate in CCM. In the islanded mode of the AC microgrid, the battery inverter operates in VCM and DG inverter operates in CCM. During grid-connected mode of the DC microgrid, the AC/DC converter connected to distribution grid 2 regulates the DC load bus voltage and all converters in the DC microgrid operate in CCM. In the islanded mode of the DC microgrid, the battery converter operates in VCM and the other converters operate in CCM. In this MMG system, the principle of control of power flows through the lines between the adjacent microgrids is based on the power flow equations (4.1) - (4.4) [103] as follows:

If $V_{1}=V_{2}=V_{3}=V$, then

$$
\begin{aligned}
& P_{s 1}=\left(V^{2} / Z_{t 1}\right) \cos \theta_{t 1}-\left(V^{2} / Z_{t 1}\right) \cos \left(\theta_{t 1}+\delta_{12}\right) \\
& P_{r 1}=\left(V^{2} / Z_{t 1}\right) \cos \left(\theta_{t 1}-\delta_{12}\right)-\left(V^{2} / Z_{t 1}\right) \cos \theta_{t 1} \\
& Q_{s 1}=\left(V^{2} / Z_{t 1}\right) \sin \theta_{t 1}-\left(V^{2} / Z_{t 1}\right) \sin \left(\theta_{t 1}+\delta_{12}\right) \\
& Q_{r 1}=\left(V^{2} / Z_{t 1}\right) \sin \left(\theta_{t 1}-\delta_{12}\right)-\left(V^{2} / Z_{t 1}\right) \sin \theta_{t 1}
\end{aligned}
$$

where $\delta_{12}=\delta_{1}-\delta_{2}=$ phase angle difference between voltages $V_{1}$ and $V_{2}$ 


$$
\begin{gathered}
\delta_{1}=\text { phase angle of voltage } V_{1} \\
\delta_{2}=\text { phase angle of voltage } V_{2} \\
Z_{t 1} \angle \theta_{t l}=\text { Impedance of line } 1
\end{gathered}
$$

Similarly, power flow equations for $P_{s 2}, P_{s 3}, P_{r 2}, P_{r 3}, Q_{s 2}, Q_{s 3}, Q_{r 2}$ and $Q_{r 3}$ can be obtained. For satisfactory operation of the MMG system, the magnitudes of voltages at load buses and at the outputs of the interconnecting inverters are maintained at nominal values [103]. Thus, control of real power transfer between adjacent microgrids in the proposed MMG system is achieved primarily through the variations in phase angles of the output voltages of the interconnecting inverters or phase angles of the AC load bus voltages.

\subsection{Modelling of Converters in the MMG System}

MPC has been gaining increasing attention in recent years for the control of power electronic devices such as converters and inverters due to its several advantages. Discrete-time implementation and fast control platforms available make MPC a suitable control technique for the converters and inverters [36]. Finite control set MPC method introduced in [36] depends on the line and load impedances of the system whereas it can be inferred from the state-space models of different converters in the following sub-sections that the proposed MPC algorithm is dependent only on the converter and inverter parameters and is independent of the line and load impedances. Thus, the proposed MPC algorithm is an ideal choice for the control of multiple converters and inverters in the proposed MMG system. The modelling of converter and inverter is explained in the following sub-sections.

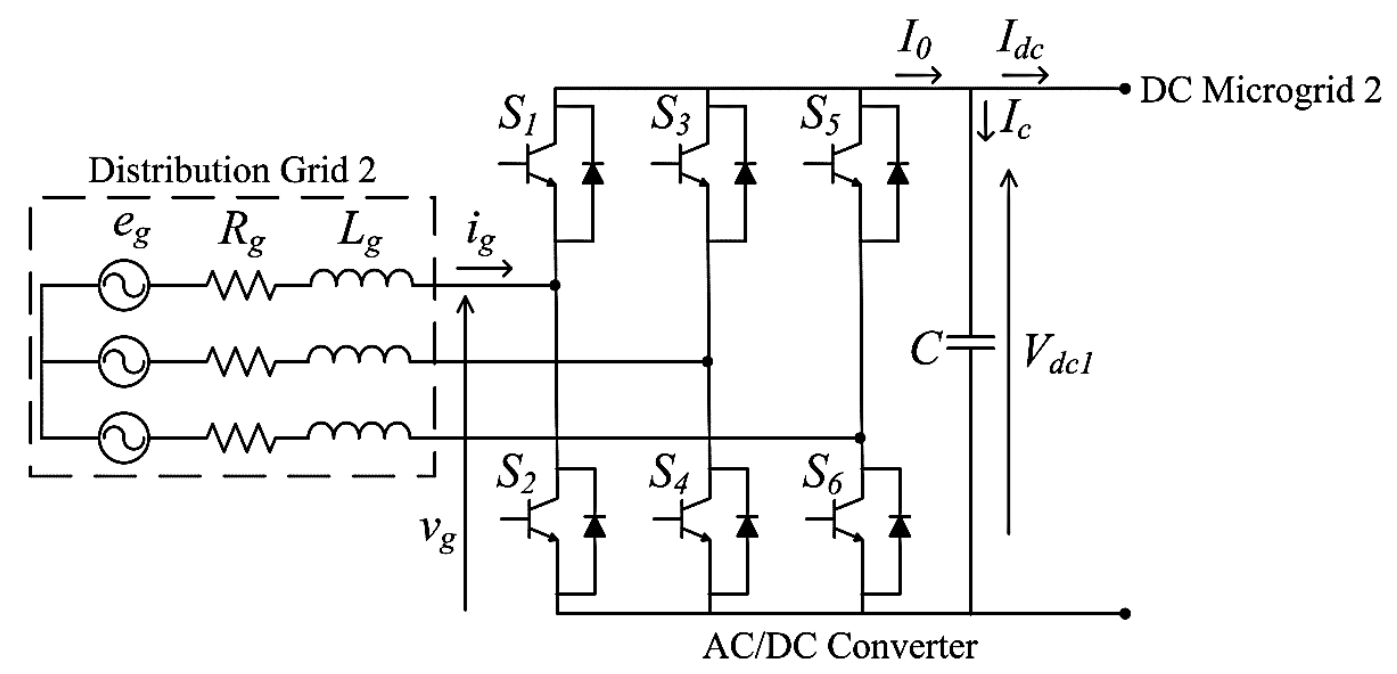

Figure 4-3: Power circuit of AC/DC converter connected to distribution grid 2. 


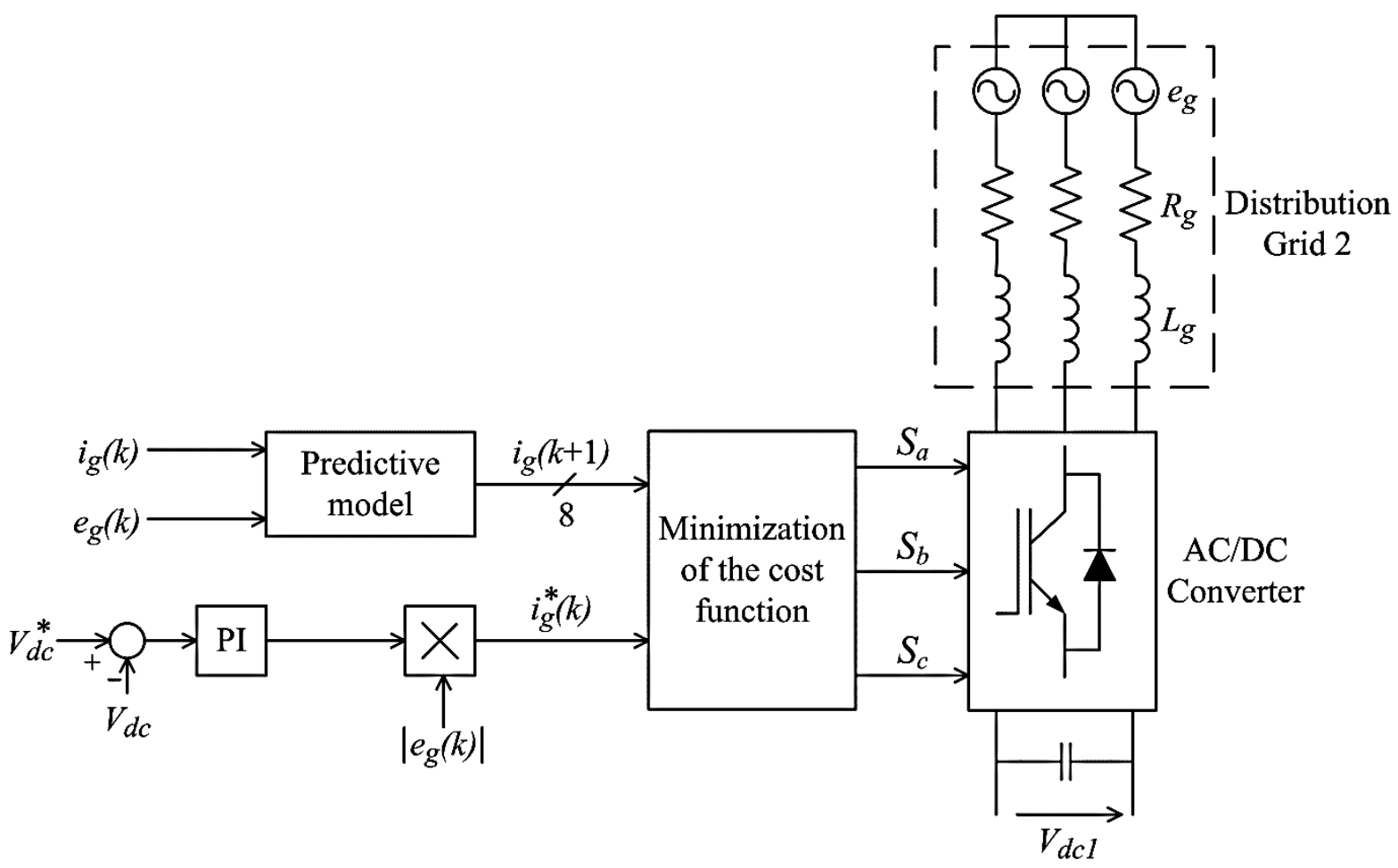

Figure 4-4: Control scheme for AC/DC converter [36].

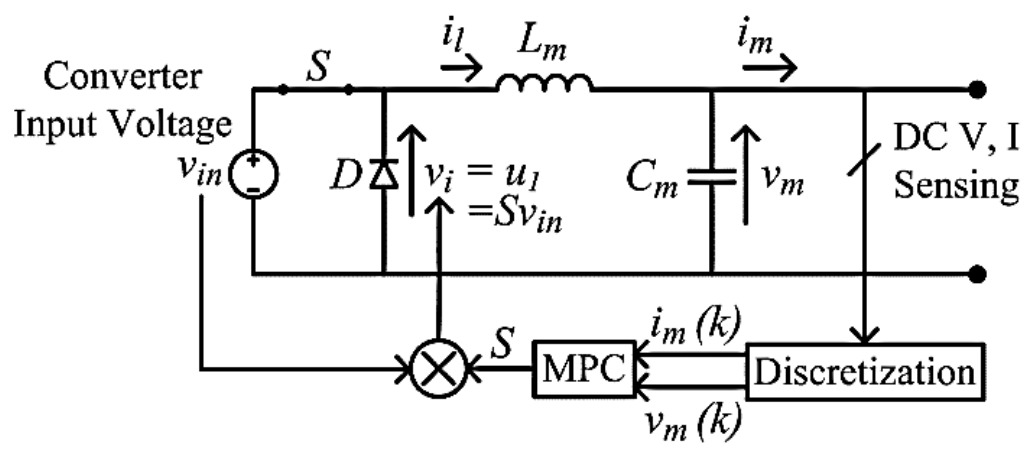

Figure 4-5: DC/DC buck converter model.

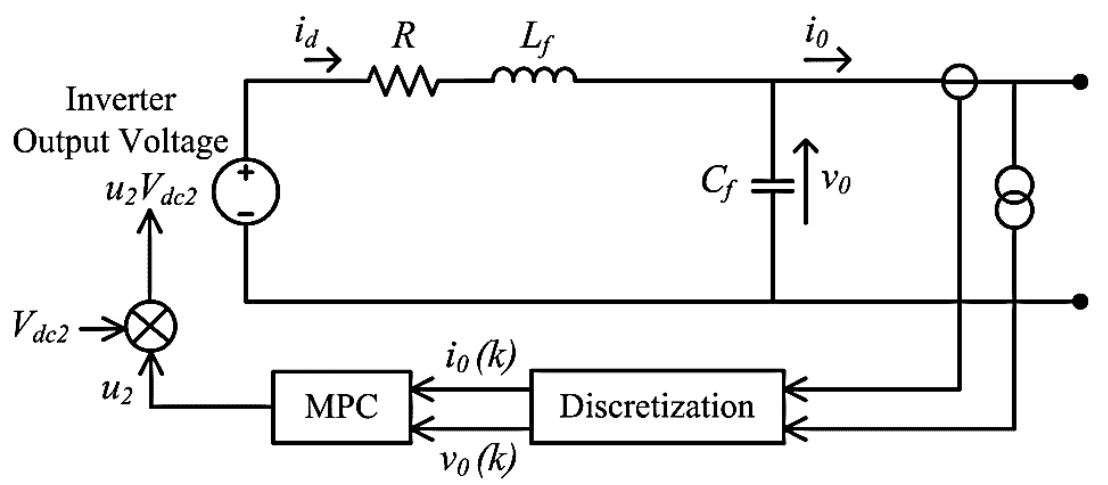

Figure 4-6: Single-phase DC/AC inverter model. 


\subsubsection{AC/DC Converter Model and Control}

Figure 4-3 shows the power circuit of AC/DC converter connected to distribution grid 2. By applying Kirchhoff's voltage law to the power circuit the following equation is obtained:

$$
e_{g}=v_{g}+R_{g} i_{g}+L_{g}\left(\frac{d i_{g}}{d t}\right)
$$

where $e_{g}$ represents the three-phase distribution grid 2 voltage, $v_{g}$ is the three-phase voltage at the input side of AC/DC converter. $R_{g}$ and $L_{g}$ are the resistance and inductance of distribution grid 2. The three-phase current supplied by distribution grid 2 is represented by $i_{g}$. Control scheme for AC/DC converter is shown in Figure 4-4. The input current of AC/DC converter is controlled using the predictive model of the current which is obtained by discretizing (4.5) for a sampling time $T_{s}$ as follows [36]:

$$
i_{g}(k+1)=\left(1-\frac{R_{g} T_{S}}{L_{g}}\right) i_{g}(k)+\frac{T_{s}}{L_{g}}\left[e_{g}(k)-v_{g}(k)\right]
$$

The three-phase switching voltage vector $v_{g}[36]$ is expressed as

$$
v_{g}=S V_{d c 1}
$$

where $S$ is the switching signal space vector defined as [36]:

$$
S=\frac{2}{3}\left(S_{a}+\alpha S_{b}+\alpha^{2} S_{c}\right) \text { where } \alpha=e^{j(2 \pi / 3)}
$$

The switching states of AC/DC converter are represented by the switching signals $S_{a}, S_{b}, S_{c}$ as follows [36]:

$S_{a}=\left\{\begin{array}{l}1, \text { if } S_{1} \text { on and } S_{2} \text { off } \\ 0, \text { if } S_{1} \text { off and } S_{2} \text { on }\end{array} ; S_{b}=\left\{\begin{array}{l}1, \text { if } S_{3} \text { on and } S_{4} \text { off } \\ 0, \text { if } S_{3} \text { off and } S_{4} \text { on }\end{array} ; S_{c}=\left\{\begin{array}{l}1, \text { if } S_{5} \text { on and } S_{6} \text { off } \\ 0, \text { if } S_{5} \text { off and } S_{6} \text { on }\end{array}\right.\right.\right.$ 
There are eight possible combinations of $S_{a}, S_{b}$, and $S_{c}$ and eight possible values of voltage vector $v_{g}$ which are same as the values given in Table 3-1 in chapter 3. The cost function $g$ to be used to calculate the absolute error between the reference current and predicted current is given as follows [36]:

$$
\begin{gathered}
\quad g=\left|i_{g}{ }^{*}(k+1)-i_{g}(k+1)\right|=\left|i_{g}{ }^{*}(k)-i_{g}(k+1)\right| \\
\text { [since } i_{g}{ }^{*}(k+1)=i_{g}{ }^{*}(k) \text { at high sampling frequency] }
\end{gathered}
$$

The predicted current in (4.6) and the cost function in (4.10) are calculated for eight different values of $v_{g}$. The voltage vector $v_{g}$ which gives the minimum value of the cost function is selected and the corresponding switching signals are generated and applied to the three-phase AC/DC converter as shown in Figure 4-4 to obtain the desired input current and thus the desired input real and reactive power. Thus, MPC uses the predictive model of the current and the required cost function to control the input current and power of the AC/DC converter. A PI controller is also used as shown in Figure 4-4 to control the DC link voltage at the output side of the AC/DC converter and to generate the input current reference [36]. The input current reference is obtained by multiplying the output of the PI controller with the magnitude of the grid voltage.

\subsubsection{DC/DC Converter Model and Control}

The DC/DC buck converter model used in DC microgrid in the MMG system is shown in Figure 4-5. The input DC voltage and diode voltage of the DC/DC buck converter are represented by $v_{i n}$ and $v_{i}$ respectively. By applying Kirchhoff's voltage and current laws to the converter model, the following equations are obtained:

$$
\begin{gathered}
L_{m}\left(d i_{l} / d t\right)=v_{i}-v_{m} \\
C_{m}\left(d v_{m} / d t\right)=i_{l}-i_{m}
\end{gathered}
$$

where $i_{l}$ is the current flowing through inductor $L_{m}, i_{m}$ is the output current and $v_{m}$ is the output voltage across capacitor $C_{m}$. 
Due to high sampling frequency, the following assumption can be made:

$$
d i_{m} / d t=0
$$

The relationship between $v_{i n}$ and $v_{i}$ can be expressed as

$$
v_{i}=S v_{i n}=u_{1}
$$

where $u_{1}$ is the control input and $S$ is the state of the switch which can be defined as follows:

$$
S=\left\{\begin{array}{l}
1, \text { switch is } \mathrm{ON} \\
0, \text { switch is } \mathrm{OFF}
\end{array}\right.
$$

By substituting (4.14) into (4.11) and rearranging (4.11)-(4.13) into a state-space form, the discrete state-space model for MPC is obtained as follows:

$$
\begin{gathered}
x_{1}(k+1)=A_{d} x_{1}(k)+B_{d} u_{1}(k) \\
y_{1}(k)=C_{d} x_{1}(k)
\end{gathered}
$$

where $x_{1}(k)=\left[\begin{array}{lll}i_{l}(k) & v_{m}(k) & i_{m}(k)\end{array}\right]^{T}$ is the state vector, $u_{1}(k)$ is the control input vector, $y_{1}(k)$ is the output vector, $T_{s}$ is sampling time, and $A_{d}, B_{d}$ and $C_{d}$ are discretized coefficients as follows:

$$
\begin{aligned}
A_{d} & =\left[\begin{array}{ccc}
1 & -T_{s} / L_{m} & 0 \\
T_{s} / C_{m} & 1 & -T_{s} / C_{m} \\
0 & 0 & 1
\end{array}\right], B_{d}=\left[\begin{array}{lll}
T_{s} / L_{m} & 0 & 0
\end{array}\right]^{T}, \\
C_{d} & =\left\{\begin{array}{lll}
{\left[\begin{array}{lll}
0 & 0 & 1
\end{array}\right] \text { in CCM }} \\
{\left[\begin{array}{lll}
0 & 1 & 0
\end{array}\right] \text { in VCM }}
\end{array}\right.
\end{aligned}
$$

From the discrete state-space model, the augmented state-space model of the DC/DC converter is obtained as follows:

$$
\begin{gathered}
X_{e}(k+1)=A_{e} X_{e}(k)+B_{e} U_{e}(k) \\
Y_{e}(k)=C_{e} X_{e}(k)
\end{gathered}
$$


where $X_{e}(k)=\left[\begin{array}{lllll}\Delta i_{l}(k) & \Delta v_{m}(k) & \Delta i_{m}(k) & i_{m}(k)\end{array}\right]^{T}$ is the state vector, $U_{e}(k)=\Delta u_{l}(k)$ is the control input vector, $Y_{e}(k)$ is the output vector which is either converter output current or voltage and the coefficients in the augmented state-space model are as follows:

$$
A_{e}=\left[\begin{array}{cccc}
1 & -T_{s} / L_{m} & 0 & 0 \\
T_{s} / C_{m} & 1 & -T_{s} / C_{m} & 0 \\
0 & 0 & 1 & 0 \\
0 & 0 & 1 & 1
\end{array}\right], B_{e}=\left[\begin{array}{c}
T_{s} / L_{m} \\
0 \\
0 \\
0
\end{array}\right], C_{e}=\left[\begin{array}{l}
0 \\
0 \\
0 \\
1
\end{array}\right]^{T}
$$

\subsubsection{DC/AC Inverter Model and Control}

The model of DG, battery and interconnecting inverters in the MMG system is shown in Figure 4-6. The discrete state-space model of the inverter is derived and then converted into augmented state-space model. By applying Kirchhoff's voltage and current laws to the single-phase inverter model in Figure 4-6, the following equations are obtained:

$$
\begin{aligned}
u_{2} V_{d c 2} & =i_{d} R+L_{f}\left(d i_{d} / d t\right)+v_{0} \\
i_{d} & =C_{f}\left(d v_{0} / d t\right)+i_{0}
\end{aligned}
$$

where $R$ represents the internal loss of the inverter, $L_{f}$ and $C_{f}$ are the inductance and capacitance of the LC filter respectively, $i_{d}$ is the current flowing through $L_{f}, i_{0}$ is the output current, $u_{2}$ is the control input, $V_{d c 2}$ is the input voltage, and $v_{0}$ is the output voltage. Due to high sampling frequency, the following assumption holds:

$$
\left(d i_{0} / d t\right)=0
$$

By rearranging (4.20)-(4.22) into a state-space form, the discrete state-space model for MPC can be obtained as follows:

$$
\begin{gathered}
x_{2}(k+1)=A_{g} x_{2}(k)+B_{g} u_{2}(k) \\
y_{2}(k)=C_{g} x_{2}(k)
\end{gathered}
$$


where $x_{2}(k)=\left[\begin{array}{lll}i_{d}(k) & v_{0}(k) & i_{0}(k)\end{array}\right]^{T}$ is the state vector, $u_{2}(k)$ is the control input vector, $y_{2}(k)$ is the output vector, $T_{s}$ is sampling time, and $A_{g}, B_{g}$ and $C_{g}$ are discretized coefficients as follows:

$$
\begin{aligned}
A_{g} & =\left[\begin{array}{ccc}
1-R T_{s} / L_{f} & -T_{s} / L_{f} & 0 \\
T_{s} / C_{f} & 1 & -T_{s} / C_{f} \\
0 & 0 & 1
\end{array}\right], B_{g}=\left[\begin{array}{lll}
V_{d c 2} T_{s} / L_{f} & 0 & 0
\end{array}\right]^{T}, \\
C_{g} & =\left\{\begin{array}{lll}
{\left[\begin{array}{lll}
0 & 0 & 1
\end{array}\right] \text { in CCM }} \\
{\left[\begin{array}{lll}
0 & 1 & 0
\end{array}\right] \text { in VCM }}
\end{array}\right.
\end{aligned}
$$

From the discrete state-space model, the augmented state-space model of the DC/AC inverter is obtained as follows:

$$
\begin{gathered}
X_{h}(k+1)=A_{h} X_{h}(k)+B_{h} U_{h}(k) \\
Y_{h}(k)=C_{h} X_{h}(k)
\end{gathered}
$$

where $X_{h}(k)=\left[\begin{array}{llll}\Delta i_{d}(k) & \Delta v_{0}(k) & \Delta i_{0}(k) & i_{0}(k)\end{array}\right]^{T}$ is the state vector, $U_{h}(k)=\Delta u_{2}(k)$ is the control input vector and $Y_{h}(k)$ is the output vector which is either inverter output current or voltage. The coefficients in the augmented state-space model are as follows:

$$
\begin{aligned}
& A_{h}=\left[\begin{array}{cccc}
1-\left(R T_{s} / L_{f}\right) & -T_{s} / L_{f} & 0 & 0 \\
T_{s} / C_{f} & 1 & -T_{s} / C_{f} & 0 \\
0 & 0 & 1 & 0 \\
0 & 0 & 1 & 1
\end{array}\right], C_{h}=\left[\begin{array}{llll}
0 & 0 & 0 & 1
\end{array}\right] \\
& B_{h}=\left[V_{d c 2} T_{s} / L_{f} \quad 0 \quad 00 \quad 0\right]^{T}
\end{aligned}
$$

\subsubsection{Objective Function and Constraints of DC/DC Converter and DC/AC Inverter}

In order to control the augmented state-space models in (4.18) and (4.25), quadratic programming is used to minimize the following objective function [109], [110]: 


$$
J=\left(R_{S}-Y\right)^{T}\left(R_{S}-Y\right)
$$

subject to the input and input increment constraints as follows:

$$
-1 \leq u_{1}(k) \leq 1 ;-1 \leq \Delta u_{1}(k) \leq 1 ;-1 \leq u_{2}(k) \leq 1 ;-1 \leq \Delta u_{2}(k) \leq 1
$$

where $R_{s}$ is the set-point matrix and $Y$ is the output of the augmented model which is equal to either $Y_{e}$ in (4.19) or $Y_{h}$ in (4.26). The objective function is used to ensure that the output of the augmented model tracks the reference with minimum error. Using the augmented models, objective functions and constraints, the MPC algorithms generate the optimal control signals $u_{1}$ and $u_{2}$ for the DC/DC converter and the DC/AC inverter respectively. Then the optimal control signals are applied to the DC/DC converter and the DC/AC inverter shown in Figure 4-5 and Figure 4-6 respectively. The predictive models of different converters in (4.6), (4.16) and (4.23) contain only parameters of the converters such as resistance, inductance and capacitance and do not contain any system parameters such as grid, line and load impedances. Thus the proposed MPC algorithm, unlike the existing MPC algorithms, is independent of grid, line and load impedances of the system considered.

\subsection{Control System of the MMG System}

The proposed control system for the MMG system is shown in Figure 4-7. It consists of a centralized controller for the MMG system and local controllers (LCs) for all the converters and inverters in each microgrid. The centralized controller collects system data such as load bus voltages, load demands and operation modes of the microgrids, etc. Based on these data, the centralized controller performs five main functions: (1) send reference real and reactive power output signals, $P_{d i(r e f)}, Q_{d i(r e f)}(i=1,3)$ and $P_{b i(r e f)}, Q_{b i(r e f)}(i=1,3)$ to DG and battery inverters' LCs respectively in grid-connected modes of AC microgrids; (2) send reference load bus voltage signals $V_{L i(r e f)} \angle \delta_{i(r e f)}(i=1,3)$ to battery inverters' LCs in islanded modes of AC microgrids; (3) send reference real power output signals $P_{d 2(r e f)}, P_{b 2(r e f)}$ and $P_{s 2(r e f)}$ to LCs of DG, battery and supercapacitor converters respectively in grid-connected mode of DC microgrid; (4) send reference load bus voltage signal $V_{L 2}$ to battery converter's LC in islanded mode of DC microgrid; and (5) send reference voltage signals $V_{n 1(r e f)}<\delta_{n 1(\text { ref })}$ and $V_{n 2(r e f)} \angle \delta_{n 2(r e f)}$ to LCs of interconnecting inverters in grid-connected and islanded modes of DC microgrid. Using the proposed MPC technique, LCs generate and send the optimal switching signals to inverters and converters. To maintain effective operation of the 
interconnected microgrids in the $\mathrm{MMG}$ system, a reliable and suitable high speed communication network between the centralized and local controllers is essential [104].

During grid-connected mode of AC microgrid in the MMG system, the DG inverter in the microgrid is controlled to deliver a predetermined amount of real and reactive power to the load and the battery inverter serves as the back-up inverter. In islanded mode of AC microgrid, centralized controller detects a frequency drop in the system and sends an appropriate voltage reference signal to the battery inverter's LC of the islanded AC microgrid, as explained in Chapter 3.

During grid-connected mode of DC microgrid, the DG converter in the microgrid is controlled to deliver a predetermined amount of real power to the load and the converters of battery and supercapacitor units serve as the back-up converters. During islanding of DC microgrid from distribution grid 2, the centralized controller detects voltage drop in DC microgrid and sends an appropriate voltage reference signal to the battery converter's LC and a power reference signal to the supercapacitor converter's LC in the islanded DC microgrid to maintain DC load bus voltage.

When DC microgrid is islanded, the phase angles of the output voltages of the interconnecting inverters are calculated based on the desired real power to be obtained at the receiving ends of the lines adjacent to the interconnecting inverters. These phase angles are calculated from (4.2) as follows:

$$
\delta_{n 1(r e f)}=\delta_{n 2(r e f)}=\delta_{12(r e f)}=\theta_{t 1}-\cos ^{-1}\left[P_{r 1(r e f)} Z_{t 1} / V^{2}+\cos \theta_{t 1}\right]
$$

where $\delta_{12(\text { ref) }}$ is the reference phase angle difference between $V_{1}$ and $V_{2}, \delta_{n 1(r e f)}$ and $\delta_{n 2(r e f)}$ are the reference phase angles of output voltages of the interconnecting inverters, $P_{r l(r e f)}$ is the reference receiving end real power to be maintained in line 1 . The corresponding sending end real power flow in line 1 , and sending and receiving end reactive power flows in line 1 are

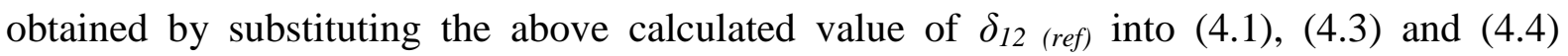
respectively.

The centralized controller monitors the load bus voltage of the DC microgrid in the MMG system and triggers undervoltage load shedding whenever the DC bus voltage deviation crosses the tolerance limit of $\pm 5 \%$ of the nominal value of $380 \mathrm{~V} .380 \mathrm{~V}$ is the standard DC 
nominal voltage currently adopted by EMERGE alliance for use in data centers, telecom systems, etc. [111]. The allowable tolerance limit for the DC bus voltage is $\pm 5 \%$ of $380 \mathrm{~V}$ [111]. An undervoltage load shedding scheme is proposed for the islanded DC microgrid [112]: (1) $15 \%$ load is shed in the islanded DC microgrid when its load bus voltage drops to $95 \%$ of the nominal value; (2) $10 \%$ additional load is shed in the islanded DC microgrid when its load bus voltage drops to $93 \%$ of the nominal value; and (3) $5 \%$ additional load is shed in the islanded DC microgrid when its load bus voltage drops below $90 \%$ of the nominal value.

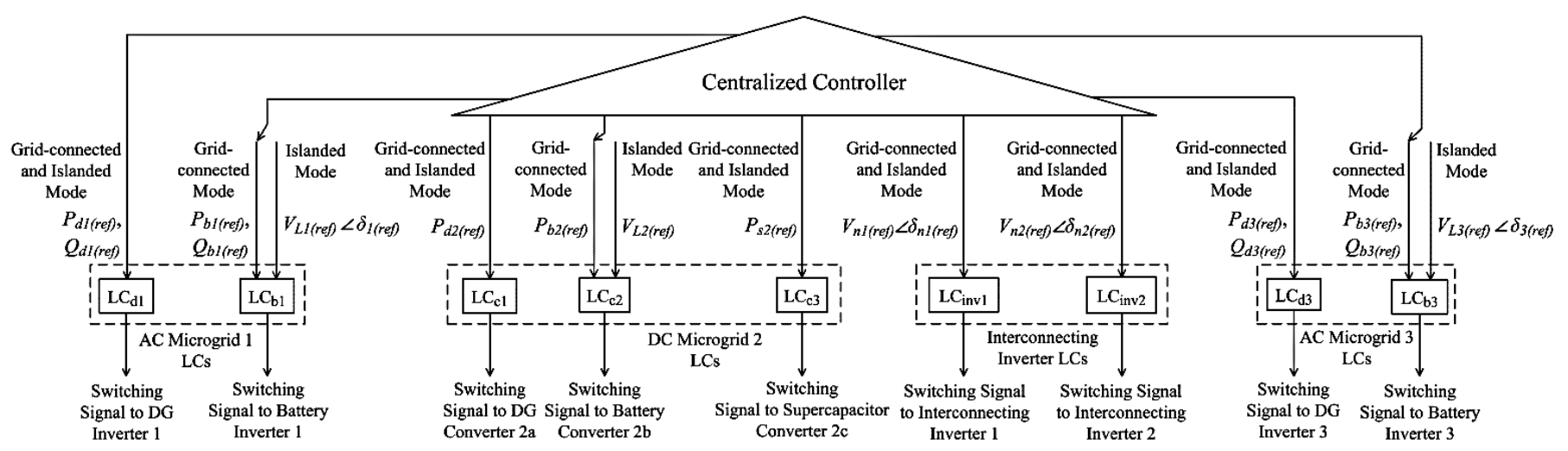

Figure 4-7: Proposed control system for AC/DC multi-area MMG system.

\subsection{Simulation Studies}

Simulation studies are conducted using MATLAB/Simulink to verify the performance of the MMG system. Three test cases are conducted to demonstrate the effective operation and control of the MMG system. The first test case implements the effective regulation of DC load bus voltage during islanding of DC microgrid in the MMG system. The second test case demonstrates the method of exchange of power between the islanded DC microgrid and adjacent grid-connected AC microgrids. The third test case demonstrates undervoltage load shedding in the islanded DC microgrid. The parameters of the proposed MMG system are shown in Table 4-1. 
Table 4-1: Parameters of the proposed system

\begin{tabular}{|c|c|}
\hline Parameter & Value \\
\hline Inverter input voltage & $V_{d c 2}=800 \mathrm{~V}$ \\
\hline Loss resistance of inverter & $R=1 \mu \Omega$ \\
\hline LC filter of inverter & $L_{f}=1 \mu \mathrm{H}, C_{f}=0.02 \mathrm{~F}$ \\
\hline DC/DC converter input voltage & $V_{\text {in }}=600 \mathrm{~V}$ \\
\hline $\mathrm{DC} / \mathrm{DC}$ converter inductor & $L_{m}=50 \mu \mathrm{H}$ \\
\hline DC/DC converter capacitor & $C_{m}=600 \mu \mathrm{F}$ \\
\hline Supply voltage of distribution grid & $e_{g}=22000 / \sqrt{3} \mathrm{Vrms}$ (phase) \\
\hline $\begin{array}{l}\text { Resistance and inductance of } \\
\text { distribution grid } 2\end{array}$ & $R_{g}=0.1 \Omega, L_{g}=1 \mathrm{mH}$ \\
\hline Line resistance and inductance & $R_{t i}=1.6 \Omega, L_{t i}=6 \mathrm{mH}(i=1,2,3)$ \\
\hline Leakage inductance of transformer & $L_{T}=21 \mu \mathrm{H}$ \\
\hline \multicolumn{2}{|l|}{ Details of different component ratings: } \\
\hline a) DG units 1 and 3 & $1 \mathrm{MW}(\max ), 1 \mathrm{MVAr}(\max )$ \\
\hline b) DG unit 2 & $1 \mathrm{MW}(\max )$ \\
\hline c) Storage battery units 1,2 and 3 & 1.1 MW (max), 1.1 MWh (max) \\
\hline d) Supercapacitor unit 2 & $2 \mathrm{MW}(\max ), 1.11 \mathrm{kWh}(\max )$ \\
\hline e) Transformers & $2.5 \mathrm{MVA}, 22 / 0.4 \mathrm{kV}$ \\
\hline f) AC Loads 1 and 3 & $2 \mathrm{MW}, 1 \mathrm{MVAr}$ \\
\hline g) DC Load 2 & $2 \mathrm{MW}$ \\
\hline
\end{tabular}

\subsubsection{Test Case 1 - Islanding of DC Microgrid}

Power flows in the MMG system during normal steady-state operation are shown in Figure 48. In the normal steady-state operation, all the microgrids in the MMG system are gridconnected and the DG units and the distribution grids meet the real and reactive power demands of the loads. Each distribution grid regulates the voltage and frequency of its respective microgrid during this operation. There are no power flows in the interconnecting lines and each distribution grid supplies $1 \mathrm{MW}$ to the load in its respective microgrid. The centralized controller always sends the desired real and reactive power reference signals to the LCs of the DG inverters and converters in the microgrids during both grid-connected and islanded conditions. DG inverter in AC microgrid supplies $1 \mathrm{MW}$ and $1 \mathrm{MVAr}$ to the load. 
Battery inverter in AC microgrid does not supply any real and reactive power initially. DG converter in DC microgrid supplies $1 \mathrm{MW}$ to the load while the converters of battery and supercapacitor units do not supply any real power initially. Thus the load demand is met in each microgrid in the MMG system during the normal steady-state operation.

The simulation begins with DC microgrid 2 operating in grid-connected mode for $0 \leq \mathrm{t}<1 \mathrm{~s}$. At $\mathrm{t}=1 \mathrm{~s}$, a three-phase balanced fault occurs at point ' $\mathrm{G}$ ' (shown in Figure 4-1) near distribution grid 2 which causes the secondary phase voltage of the distribution grid 2 transformer to sag severely from $230 \mathrm{~V}(\mathrm{rms})$ to $11.5 \mathrm{~V}(\mathrm{rms})$ for $1 \leq \mathrm{t}<1.2 \mathrm{~s}$ as shown in Figure 4-11. As a result, the AC/DC converter connected to distribution grid 2 is unable to control DC load 2 bus voltage and the power supplied by distribution grid 2 reduces to almost 0 . In the DC microgrid, this real power shortage causes the DC load bus voltage to drop [113]. Thus, at the instant of fault, the DC load 2 bus voltage drops from its nominal value of $380 \mathrm{~V}$ to $373 \mathrm{~V}$ at $\mathrm{t}=1 \mathrm{~s}$ as shown in Figure 4-12. However, the supercapacitor converter 2c operating in CCM is tasked by the centralized controller to quickly give a power output of 1 MW for a very short duration of $0.2 \mathrm{~s}$ for $1 \leq \mathrm{t} \leq 1.2 \mathrm{~s}$ as shown in Figure 4-13 until the battery converter $2 \mathrm{~b}$ operating in VCM ramps up its power output from $0 \mathrm{MW}$ to $1 \mathrm{MW}$ for 1 $\leq \mathrm{t} \leq 1.2 \mathrm{~s}$ as shown in Figure 4-14. Since the supercapacitor converter 2c supplies power instantaneously at $\mathrm{t}=1 \mathrm{~s}$, the DC load 2 bus voltage is quickly restored to its nominal value of $380 \mathrm{~V}$ for $\mathrm{t} \geq 1.1 \mathrm{~s}$ as observed in Figure 4-12. But at $\mathrm{t}=1.2 \mathrm{~s}$, the DC load 2 bus voltage drops to $370 \mathrm{~V}$ due to opening of the circuit breakers at both the primary and secondary sides of the distribution grid 2 transformer. When these breakers are opened, the fault is cleared and DC microgrid 2 is islanded from distribution grid 2. Since the battery converter $2 b$ delivers a steady-state power of $1 \mathrm{MW}$ for $\mathrm{t} \geq 1.2 \mathrm{~s}$ onwards, the DC load 2 bus voltage is restored to $380 \mathrm{~V}$ for $\mathrm{t} \geq 1.3 \mathrm{~s}$. Power flows in the MMG system during islanding of DC microgrid 2 are shown in Figure 4-9. Thus, this test case shows the coordinated control of supercapacitor and battery converters in the DC microgrid by the centralized controller to mitigate the voltage dips during fault and islanding of DC microgrid in the proposed MMG system. 


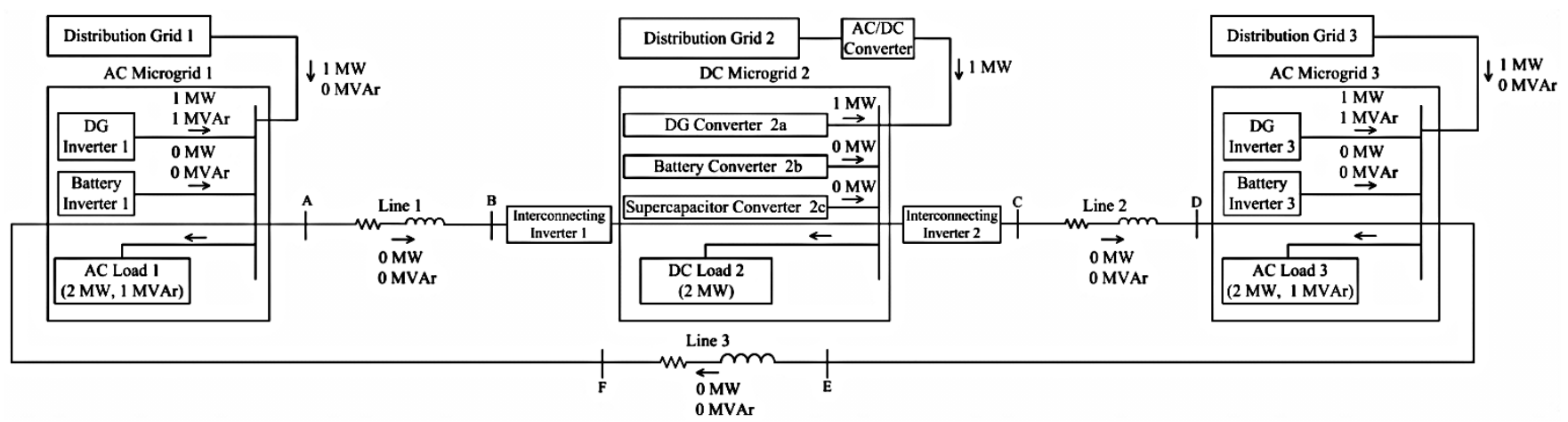

Figure 4-8: Power flows in the MMG system during steady-state operation.

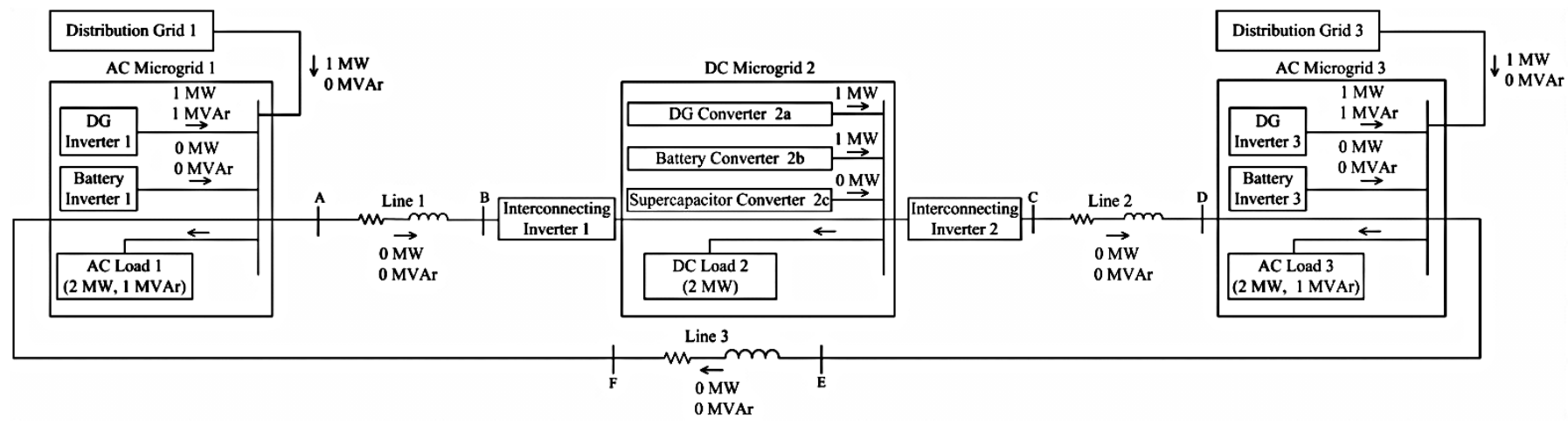

Figure 4-9: Power flows in the MMG system during islanding of DC microgrid 2.

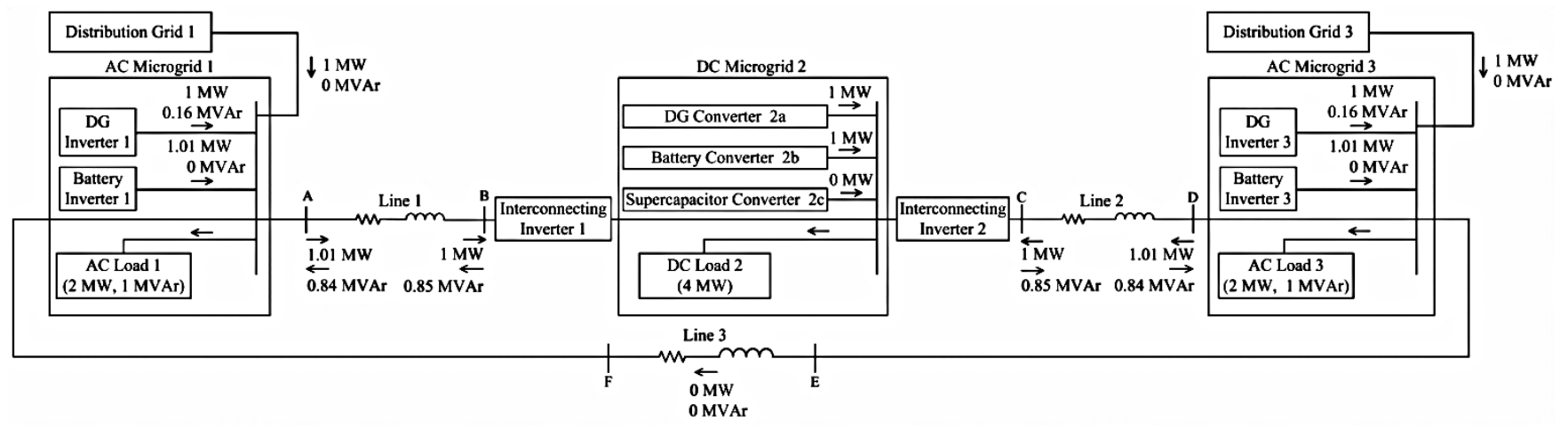

Figure 4-10: Power exchange in the MMG system during load increase in DC microgrid 2.

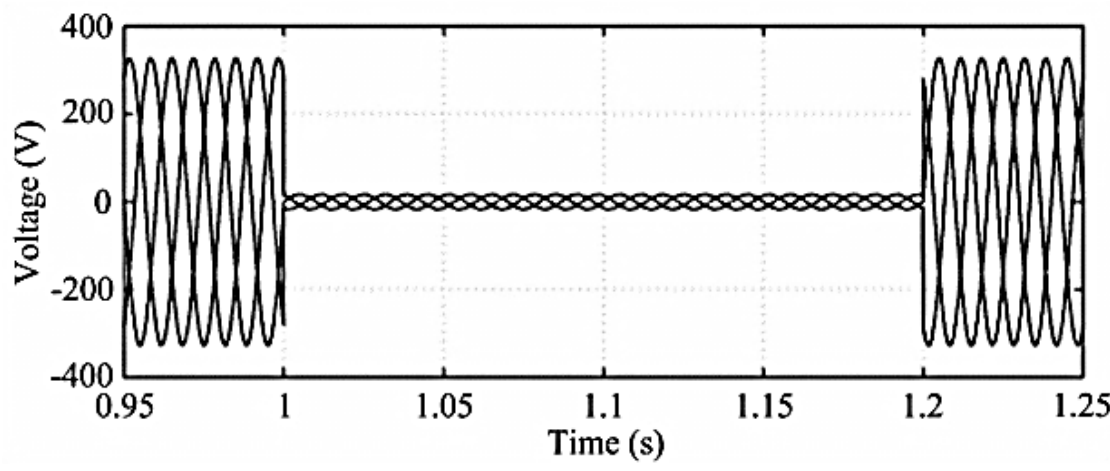

Figure 4-11: Secondary phase voltage at point ' $G$ ' in the MMG system. 


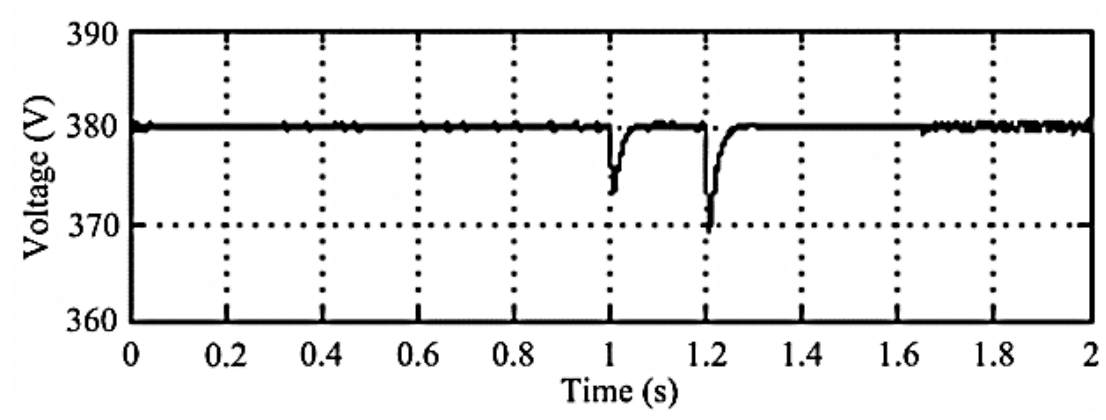

Figure 4-12: DC load 2 bus voltage.

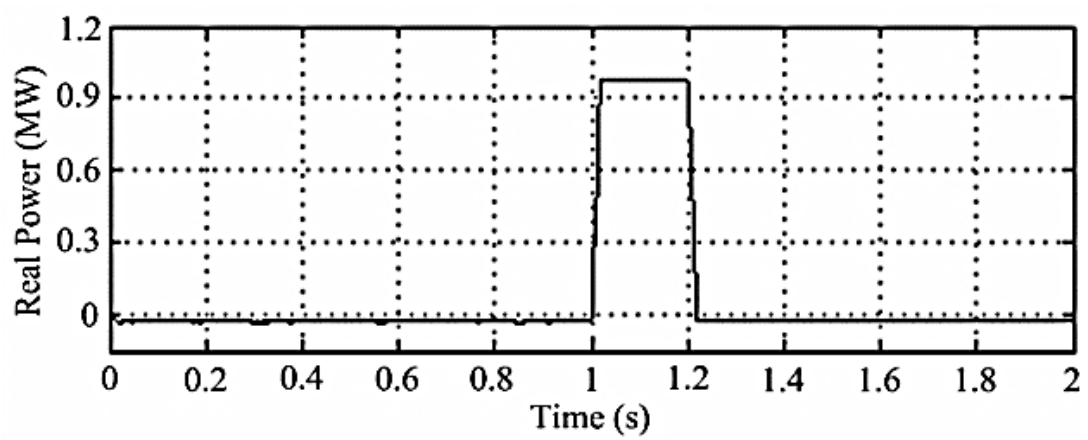

Figure 4-13: Real power output of supercapacitor converter 2c.

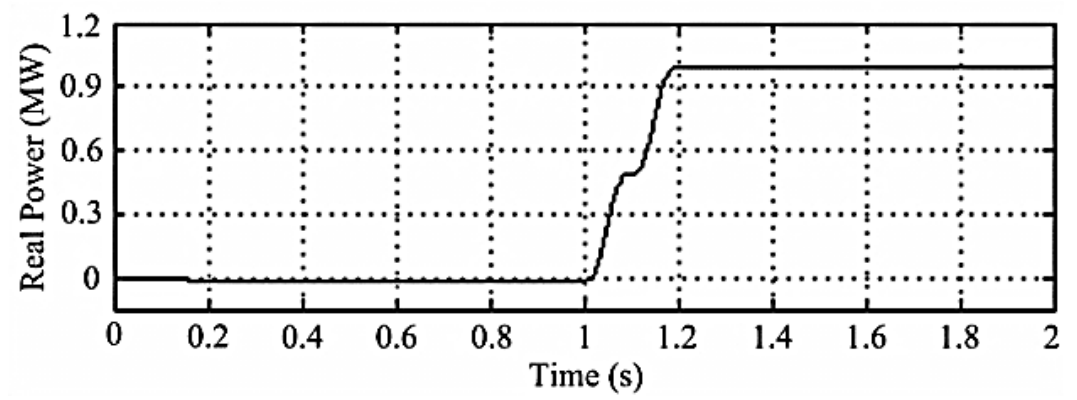

Figure 4-14: Real power output of battery converter $2 b$.

\subsubsection{Test Case 2 - Power Exchange between DC and AC Microgrids}

In the islanded DC microgrid 2, an increase of DC load from $2 \mathrm{MW}$ to $4 \mathrm{MW}$ occurs at $\mathrm{t}=3 \mathrm{~s}$ in DC microgrid 2 as shown in Figure 4-10. Although the supercapacitor unit in DC microgrid 2 can give a real power output of $2 \mathrm{MW}$ for a very short duration, the DG and battery units in DC microgrid 2 are operating at their maximum powers of $1 \mathrm{MW}$. Thus, DC microgrid 2 will not be able to meet the increase in its local load demand and as a result, the DC load 2 bus voltage will drop. In order to restore the real power balance and mitigate this voltage drop, DC microgrid 2 takes $1 \mathrm{MW}$ each from microgrids 1 and 3 in accordance with the contract agreement among the microgrids as shown in Figure 4-10. This power exchange is achieved using the proposed control system explained in Section 4.4. To achieve this desired power exchange between the microgrids, the reference phase angles of the output voltages of the interconnecting inverters 1 and 2 are calculated from (4.29). Then the 
centralized controller sends the voltage reference $230 \angle-0.5^{\circ}$ to the LCs of the interconnecting inverters. The magnitudes of the output voltages of the interconnecting inverters are always maintained at $230 \mathrm{~V}$ (rms) as shown in Figure 4-15. The phase angles of the output voltages of the interconnecting inverters are thus maintained at $-0.5^{\circ}$ for $\mathrm{t}>3 \mathrm{~s}$ as shown in Figure 416. When the phase angles of the output voltages of the interconnecting inverters are changed, the real and reactive power flows through lines 1 and 2 are increased according to (4.1), (4.2) (4.3) and (4.4). In lines 1 and 2, the desired sending and receiving end real powers equal to 1.01 MW and 1 MW respectively are shown in Figure 4-17. Also, in lines 1 and 2, the reactive powers thus obtained at the sending and receiving ends equal to 0.84 MVAr and 0.85 MVAr respectively are shown in Figure 4-18. Supercapacitor converter 2c and battery inverters 1 and 3 are thus tasked by the centralized controller to supply the required real powers to meet the load demand in islanded DC microgrid 2. Since battery inverters 1 and 3 take around $0.2 \mathrm{~s}$ to ramp up their power outputs, the supercapacitor converter $2 \mathrm{c}$ operating in $\mathrm{CCM}$ supplies real power output of $2 \mathrm{MW}$ for $0.2 \mathrm{~s}$ from $\mathrm{t}=3 \mathrm{~s}$ to $\mathrm{t}=3.2 \mathrm{~s}$ as shown in Figure 4-19.

To supply the required real power through lines 1 and 2, the real power outputs of both battery inverters 1 and 3 are controlled to increase from $0 \mathrm{MW}$ to $1.01 \mathrm{MW}$ for $\mathrm{t}>3 \mathrm{~s}$ as shown in Figure 4-20. The real power output of battery converter $2 \mathrm{~b}$ is maintained at $1 \mathrm{MW}$ itself as in test case 1. The interconnecting inverters supply the required receiving end reactive powers in lines 1 and 2 . In order to satisfy the reactive power balance in AC microgrids 1 and 3, the reactive power outputs of DG inverters 1 and 3 are decreased by the centralized and local controllers from 1 MVAr to 0.16 MVAr during load increase in DC microgrid 2 as shown in Figure 4-10. The magnitude and direction of real and reactive power flows in the MMG system during load increase in DC microgrid 2 are clearly shown in Fig. 4-10.

MPC controllers for these battery converter and inverters require only a period of about 10 cycles to track the real power reference signals of the order of MW during the initialization period. It can be observed that the proposed MPC controller is able to quickly track and settle to the power references. When there is a load increase in islanded DC microgrid 2, a power imbalance is observed in DC microgrid 2 which causes the DC load 2 bus voltage to drop to $363 \mathrm{~V}$ at $\mathrm{t}=3 \mathrm{~s}$ as seen in Figure 4-21. When this voltage drop is detected by the centralized controller, the supercapacitor converter and the battery inverters and converter are tasked to supply the required real power at $\mathrm{t}=3 \mathrm{~s}$, as mentioned earlier, to meet the load demand in DC 
microgrid 2 and to restore the DC load 2 bus voltage. Thus, for $t>3.1 \mathrm{~s}$, the DC load 2 bus voltage is restored to its nominal value of $380 \mathrm{~V}$ as shown in Figure 4-21 and thus the voltage stability can be guaranteed in the DC microgrid in the proposed MMG system.

The main characteristics which differentiate lines 1 and 2 are line impedances. For simulation purpose, the impedances of the lines 1 and 2 are assumed to be the same. But, even if the impedances of the lines are different, the proposed MMG system models and the MPC control method remain valid under this condition.

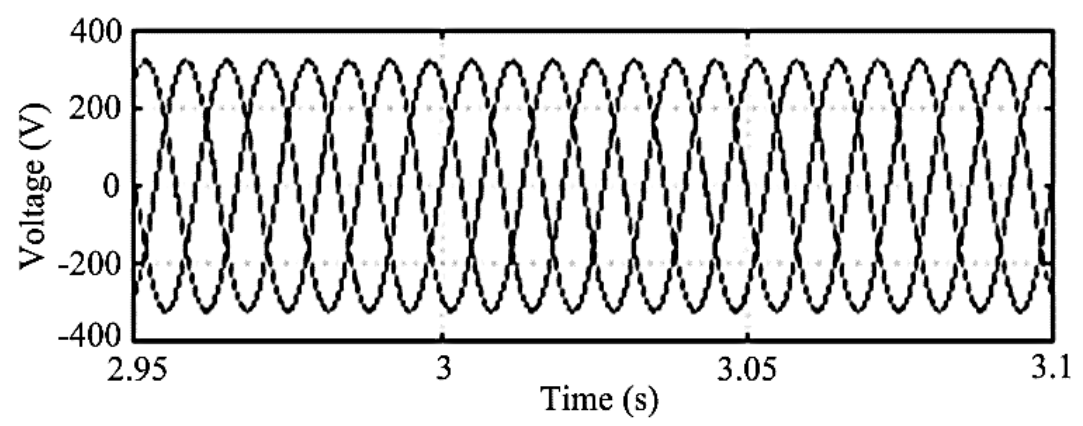

Figure 4-15: Three-phase output voltage of the interconnecting inverters 1 and 2.

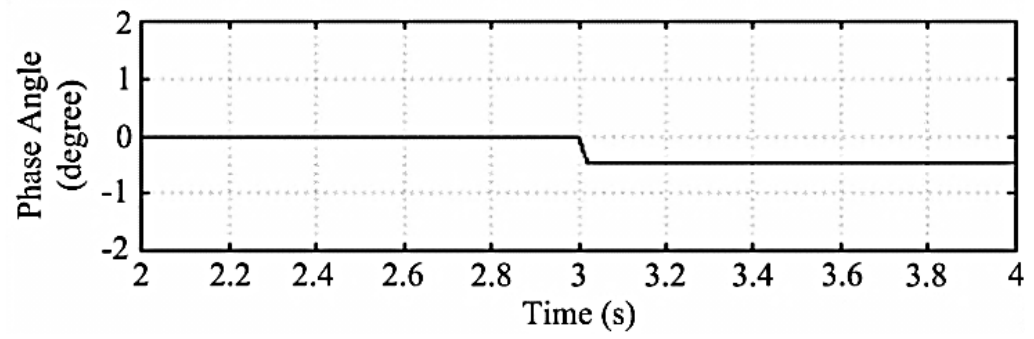

Figure 4-16: Phase angles of output voltages of the interconnecting inverters 1 and 2.

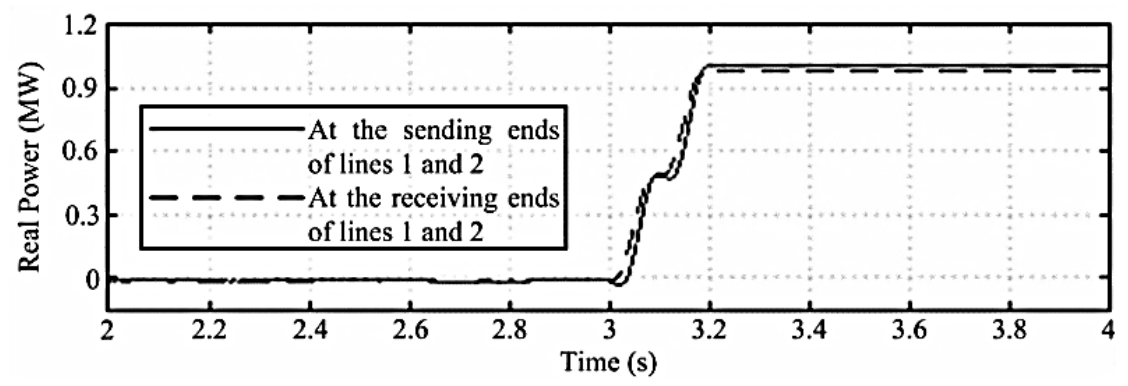

Figure 4-17: Real powers at the sending and receiving ends of lines 1 and 2. 


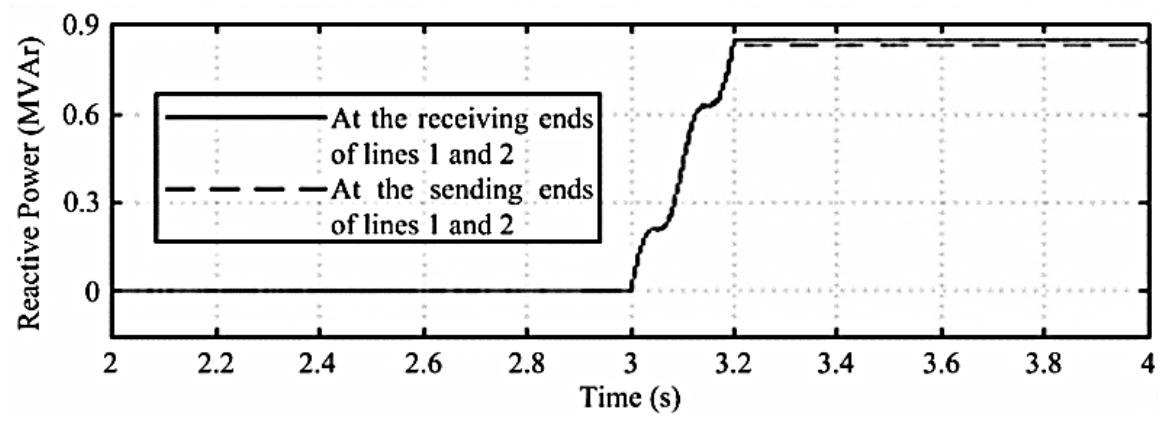

Figure 4-18: Reactive powers at the sending and receiving ends of lines 1 and 2.

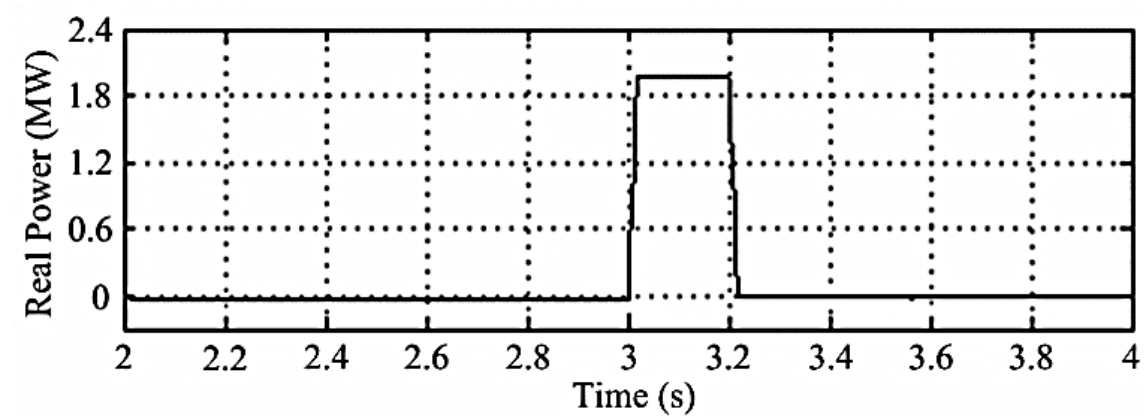

Figure 4-19: Real power output of supercapacitor converter 2c.

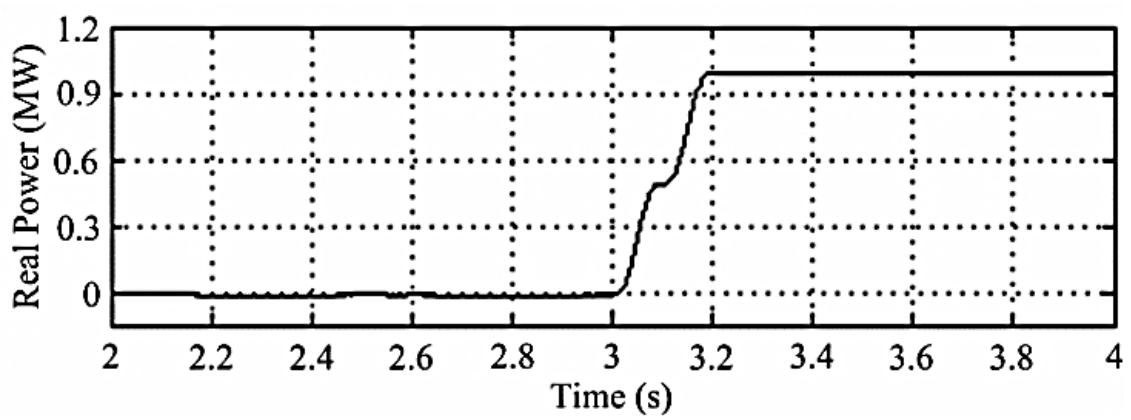

Figure 4-20: Real power outputs of battery inverters 1 and 3.

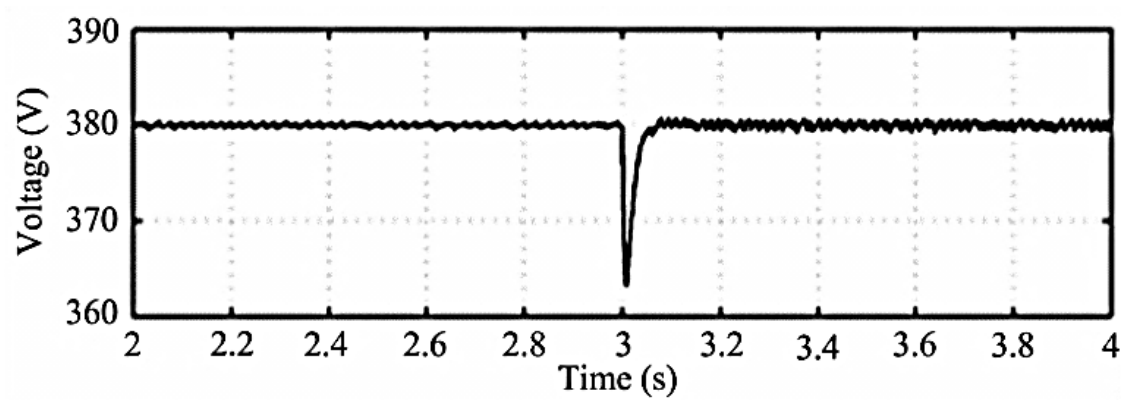

Figure 4-21: DC load 2 bus voltage. 


\subsubsection{Test Case 3 - Undervoltage Load Shedding in Islanded DC Microgrid}

At $t=8 \mathrm{~s}$, DG converter 2a in the DC microgrid fails to operate as observed in Figure 4-22, thereby resulting in a loss of $1 \mathrm{MW}$ in the islanded DC microgrid 2 in the MMG system shown in Figure 4-10. Since all the battery inverters and converter are operating at their maximum powers, supercapacitor converter $2 \mathrm{c}$ is tasked by the centralized controller at $\mathrm{t}=8$ $\mathrm{s}$ to deliver the required power of $1 \mathrm{MW}$ at rated energy for a fixed discharge time duration of supercapacitor unit 2, which is 4 s (i.e., in this case, discharge duration of supercapacitor unit $2=$ rated energy of supercapacitor unit 2/power delivered by supercapacitor unit $2=0.00111$ $\mathrm{MWh} / 1 \mathrm{MW}=0.00111 \mathrm{~h}=4 \mathrm{~s}$ ). Thus, supercapacitor converter $2 \mathrm{c}$ delivers $1 \mathrm{MW}$ for $8 \leq \mathrm{t} \leq$ $12 \mathrm{~s}$ as shown in Figure 4-23. Due to the delivery of the required power by supercapacitor converter $2 \mathrm{c}$, the DC load 2 bus voltage which drops to $374 \mathrm{~V}$ at $\mathrm{t}=8.3 \mathrm{~s}$ is restored back to $380 \mathrm{~V}$ for $\mathrm{t}>9 \mathrm{~s}$ and is maintained at $380 \mathrm{~V}$ for $9 \leq \mathrm{t} \leq 12 \mathrm{~s}$ as shown in Figure 4-24. When supercapacitor unit 2 is discharged completely for $\mathrm{t}>12 \mathrm{~s}$, a power deficit occurs in DC microgrid 2. As a result, the DC load 2 bus voltage starts to drop to a very low value beyond the tolerance limit of $\pm 19 \mathrm{~V}$ as shown in Figure 4-24. The zoom-in DC load 2 bus voltage for $12 \leq \mathrm{t} \leq 12.9 \mathrm{~s}$ is shown in Figure 4-25.

To restore the DC load 2 bus voltage, undervoltage load shedding explained in Section 4.4 is thus implemented by the centralized controller as follows: (1) when the DC load 2 bus voltage drops to $361 \mathrm{~V}$ at $\mathrm{t}=12.1 \mathrm{~s}$ as shown in Figure 4-25, 15\% of load (i.e., $15 \%$ of $4 \mathrm{MW}$ $=0.6 \mathrm{MW}$ ) is shed in DC microgrid 2 as shown in Figure 4-26; and (2) when the DC load 2 bus voltage drops to $354 \mathrm{~V}$ at $\mathrm{t}=12.2 \mathrm{~s}$ as shown in Figure 4-25, 10\% of load (i.e., 10\% of 4 $\mathrm{MW}=0.4 \mathrm{MW}$ ) is shed in DC microgrid 2 as shown in Figure 4-26. As a result, DC load 2 bus voltage is restored to its nominal value of $380 \mathrm{~V}$ for $\mathrm{t} \geq 13.5 \mathrm{~s}$ as observed in Figure 4-24. Different stages of load shedding are initiated here within a very short time delay of $0.1 \mathrm{~s}$, which is the same as the time delay used in the load shedding scheme adopted in [108]. Also, the DC voltage dip magnitude and duration in this test case are within the allowable tolerance limits in accordance with the new DC version of CBEMA (Computer Business Equipment Manufacturers Association) curve [114]. 


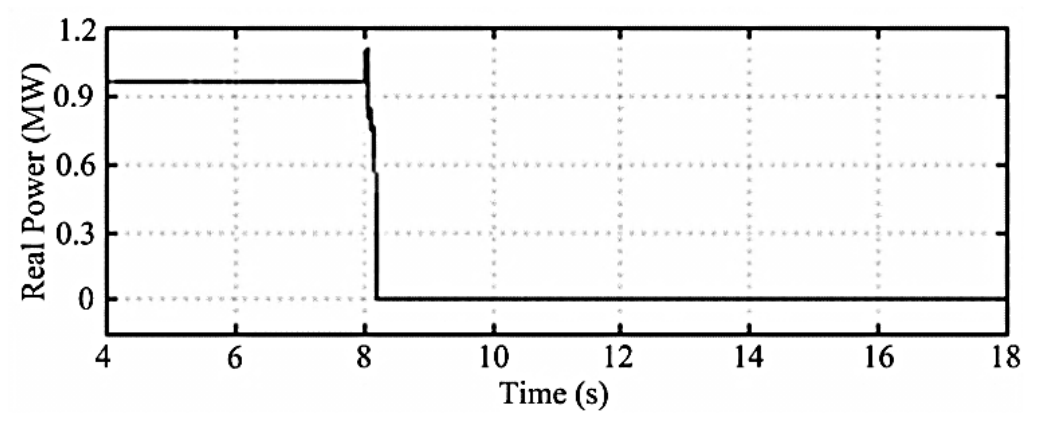

Figure 4-22: Real power output of DG converter $2 \mathrm{a}$.

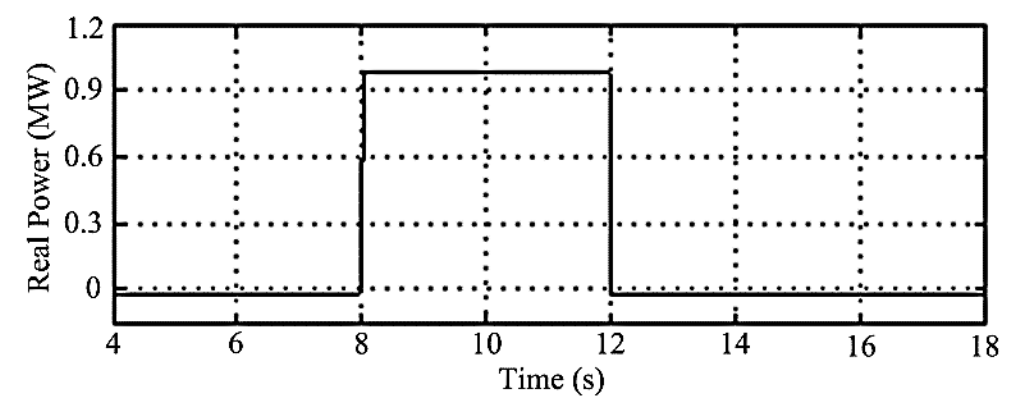

Figure 4-23: Real power output of supercapacitor converter 2c.

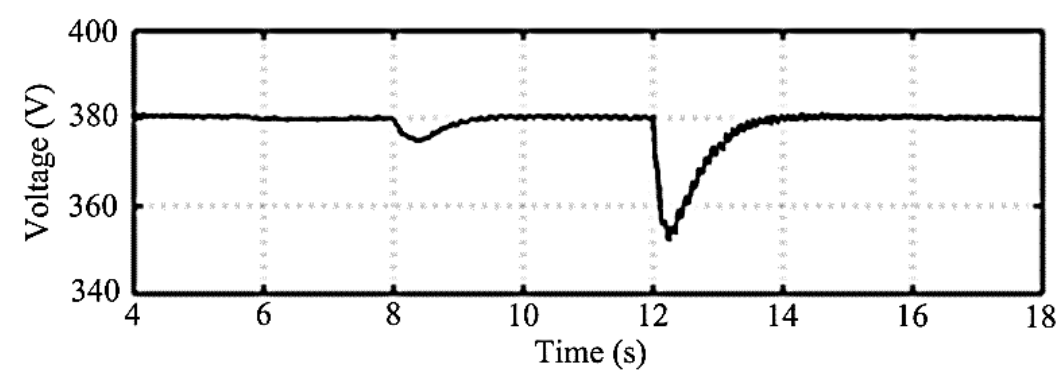

Figure 4-24: DC load 2 bus voltage.

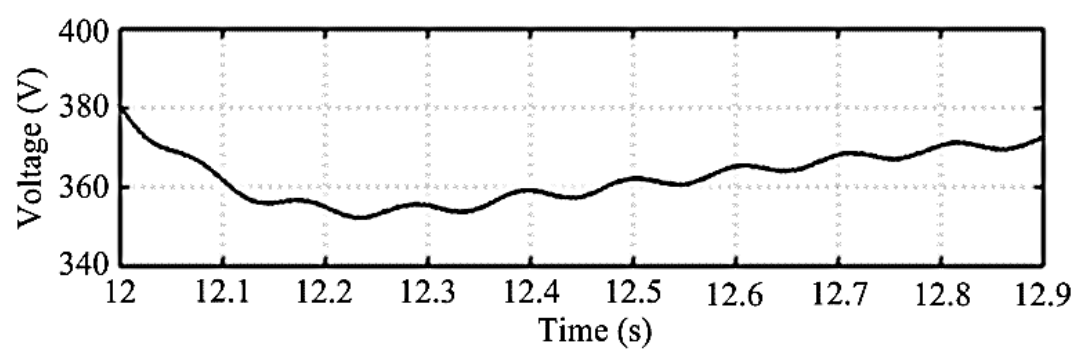

Figure 4-25: Zoom-in DC load 2 bus voltage for $12 \leq \mathrm{t} \leq 12.9 \mathrm{~s}$.

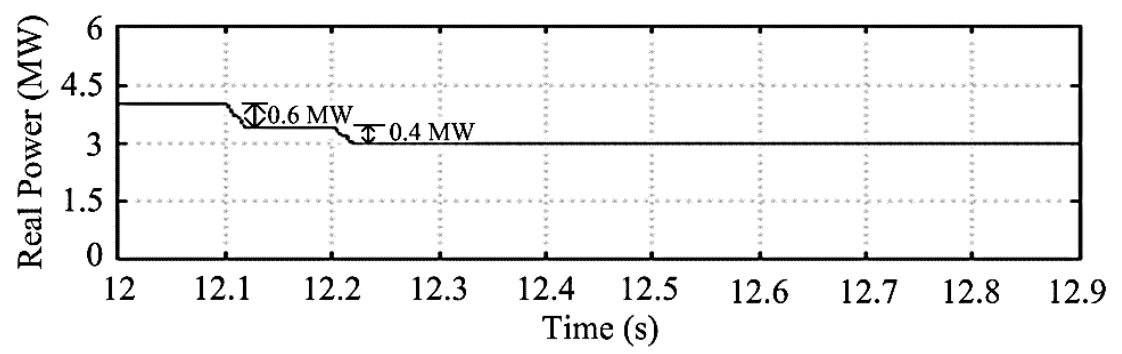

Figure 4-26: Real power consumed by DC load 2 for $12 \leq \mathrm{t} \leq 12.9 \mathrm{~s}$. 


\subsection{Conclusion}

In this chapter, a control system for an AC/DC multi-area MMG system has been presented to solve the problems of power imbalance and DC voltage variation during different operating conditions of the DC microgrid such as islanding, load increase and load shedding in the proposed MMG system. A state-space model based MPC algorithm has also been developed for the control of multiple converters and inverters in the proposed MMG system. Simulation studies have been conducted to validate the effectiveness of the proposed control system. The simulation results have shown that the developed control system can achieve good performance and the MMG system is able to operate efficiently and accurately during islanding, load increase and load shedding. In addition, the developed control system in the MMG system can facilitate desired power exchange among AC and DC microgrids under various operating conditions and can achieve effective voltage regulation during DC microgrid islanding. Thus an effectively controlled AC/DC multi-area MMG system can fulfill basic objectives such as improved reliability and security of supply, effective dynamic islanding and enhanced voltage stability. The proposed design concept for the MMG system serves as a fundamental step for the hardware implementation and validation of the MMG system, which will have a scope for further research in the area of multi-area multimicrogrids. Further discussion of different power quality issues and power quality improvement methods in the proposed MMG system is given in detail in the next chapter. 


\section{CHAPTER 5 SERIES-SHUNT NETWORK DEVICE FOR AC/AC MULTI-AREA MMG SYSTEM}

\subsection{Introduction}

This chapter presents a new power quality improvement scheme for an AC/AC multi-area MMG system. A new method of non-local harmonic current and reactive power compensation using a series-shunt network device (SSND) is proposed. Even though local harmonic current and reactive power compensation methods are available, non-local compensation methods have several advantages over local compensation methods as mentioned in Section 2.10.4 in Chapter 2. Thus, these non-local compensation methods are alternatives to be necessarily considered in the future for large MMG systems and distribution systems, which consist of widely dispersed loads. In this non-local compensation method, the compensator, namely SSND, unlike other local compensators, can be installed at some distance away from the loads. A state-space model based MPC algorithm is used for the proposed power quality improvement scheme in the proposed AC/AC multi-area MMG system to regulate various parameters such as output voltage, current and power of the inverters in SSND and in the interconnected microgrids. The use of SSND helps in the accomplishment of the following tasks in the proposed MMG system:

- Harmonic current and reactive power compensation.

- Isolation of one microgrid from voltage disturbances such as voltage distortion, voltage sag and voltage unbalance occurring in adjacent distribution grid or microgrid.

- Real power support during islanding of a microgrid.

The operation of SSND is analyzed and studied under several test case scenarios. The simulation test results show that SSND can operate effectively using MPC in the MMG system to achieve effective local and non-local harmonic current and reactive power compensation.

\subsection{System Description}

The overall configuration of the proposed SSND and MMG system is shown in Figure 5-1. The operation of SSND is tested by connecting it to a line interconnecting two microgrids as 
shown. SSND consists of a combination of shunt and series inverters. One inverter is connected in shunt with the line, while the other is connected in series with the line through an injection transformer. The dc terminals of these inverters are connected together and their dc link voltage $V_{d c}$ is supported by a common capacitor bank. The storage battery unit together with the bi-directional DC/DC converter maintains the dc link voltage of the series and shunt inverters at a constant value and also provides real power, if required. Each microgrid consists of a DG unit, a linear load and a non-linear load and is connected to its respective distribution grid through a $0.4 / 22 \mathrm{kV}$ distribution transformer. The DC output of the DG unit is connected to the load bus through a DC/DC boost converter and a DG DC/AC inverter. The DC/DC boost converter maintains the DC input voltage of the DC/AC inverter constant. Microgrids 1 and 2 are interconnected with each other through a line of reactance $X_{T}$ and inductance $L_{T}$. It is assumed that the main distribution grid is sectionalized into distribution grid 1 and distribution grid 2. The load bus voltages in microgrids 1 and 2 are represented by $V_{L 1}$ and $V_{L 2}$ respectively. The real power outputs of DG inverters 1 and 2 are represented as $P_{d 1}$ and $P_{d 2}$ respectively. The real powers supplied by distribution grids 1 and 2 are represented as $P_{g 1}$ and $P_{g 2}$ respectively. The real and reactive powers consumed by linear load 1 are represented as $P_{l l}$ and $Q_{l l}$ respectively. Similarly, the real and reactive powers consumed by linear load 2 are represented as $P_{l 2}$ and $Q_{l 2}$ respectively. The real and reactive powers consumed by non-linear load 1 are represented as $P_{n 1}$ and $Q_{n 1}$ respectively. Similarly, the real and reactive powers consumed by non-linear load 2 are represented as $P_{n 2}$ and $Q_{n 2}$ respectively. The bus voltages at the sending end and receiving end buses of the line are represented as $V_{S}$ and $V_{R}$ respectively. The real and reactive powers at the sending end of the line are represented as $P_{S}$ and $Q_{S}$ respectively. The real and reactive powers at the receiving end of the line are represented as $P_{R}$ and $Q_{R}$ respectively. In the SSND, the real and reactive powers supplied by the shunt inverter are represented as $P_{s h}$ and $Q_{s h}$ respectively. Similarly, the real and reactive powers supplied by the series inverter are represented as $P_{s e}$ and $Q_{s e}$ respectively. The voltage injected by the series inverter is represented as $V_{q}$.

Each inverter in the proposed system can operate in two different modes, namely CCM and VCM. In CCM, the output current of the inverter is regulated at the desired value and in VCM, the output voltage of the inverter is regulated at the desired value. Also, each microgrid in the proposed system basically operates in two different modes of operation: (1) grid-connected mode when the microgrid is connected to its respective distribution grid; and (2) islanded mode when the microgrid is disconnected from its respective distribution grid. 
During grid-connected operation of a microgrid in the proposed system, the distribution grid regulates the load bus voltage and frequency, and thus DG inverter in the microgrid operates in CCM. During islanding of a microgid from its respective distribution grid, the DG inverter in the islanded microgrid operates in VCM to control its load bus voltage. The DG inverters are required to supply only real power and no reactive power since SSND meets the entire reactive power demand of the MMG system. Shunt and series inverters of SSND are always operated independently in CCM and VCM respectively during both grid-connected and islanded modes of the microgrids. The shunt inverter is mainly used for reactive power compensation in both the microgrids during grid-connected and islanded modes of operation. In addition, the shunt inverter can provide for the shortage in real power in an islanded microgrid with the help of its storage battery unit. The main function of the series inverter is to isolate one microgrid from voltage disturbances such as voltage distortion, voltage sag and voltage unbalance originating in the other microgrid. SSND can also deliver real power to the microgrids during peak periods when the cost of generation from the distribution grid is high. When the storage battery unit of SSND is depleted of its energy, distribution grid 1 delivers additional real power through the shunt inverter to charge the DC link capacitor bank and thus maintain the dc-link voltage at a constant value [115]. In Figure 5-2, the detailed configuration of the three-phase SSND is shown. $V_{q, a}, V_{q, b}$ and $V_{q, c}$ represent the three-phase injection voltages. $L_{s h, a}, L_{s h, b}$ and $L_{s h, c}$ represent the three-phase filter inductances of the shunt inverter. $C_{s h, a}, C_{s h, b}$ and $C_{s h, c}$ represent the three-phase filter capacitances of the shunt inverter. $L_{s e, a}, L_{s e, b}$ and $L_{s e, c}$ represent the three-phase filter inductances of the series inverter. $C_{s e, a}, C_{s e, b}$ and $C_{s e, c}$ represent the three-phase filter capacitances of the series inverter.

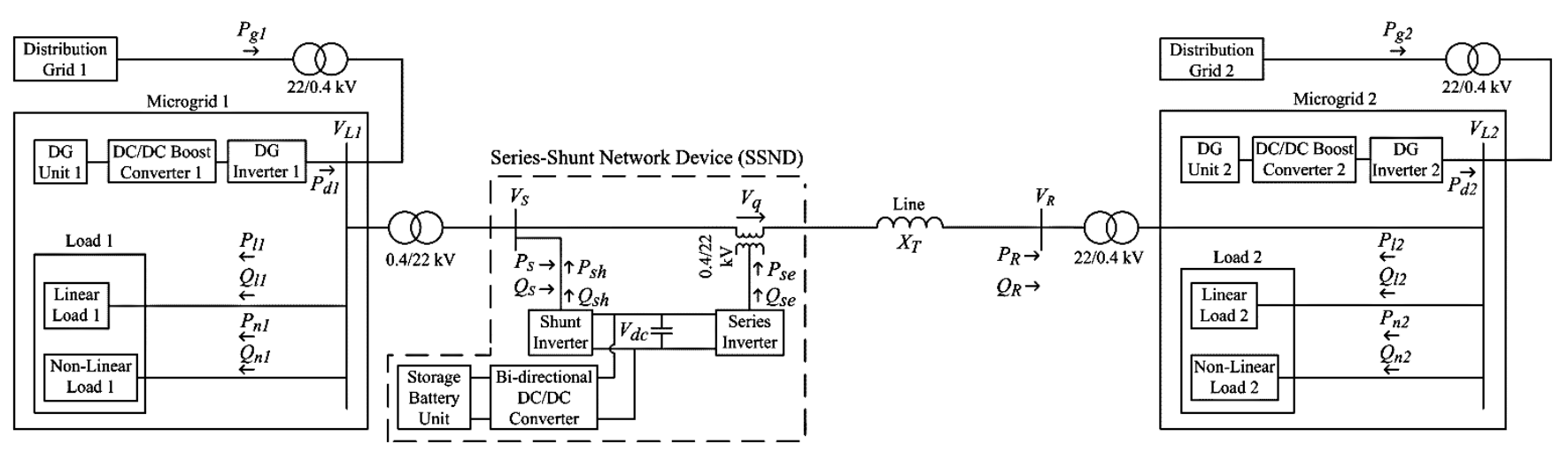

Figure 5-1: Overall configuration of the proposed SSND and MMG system. 


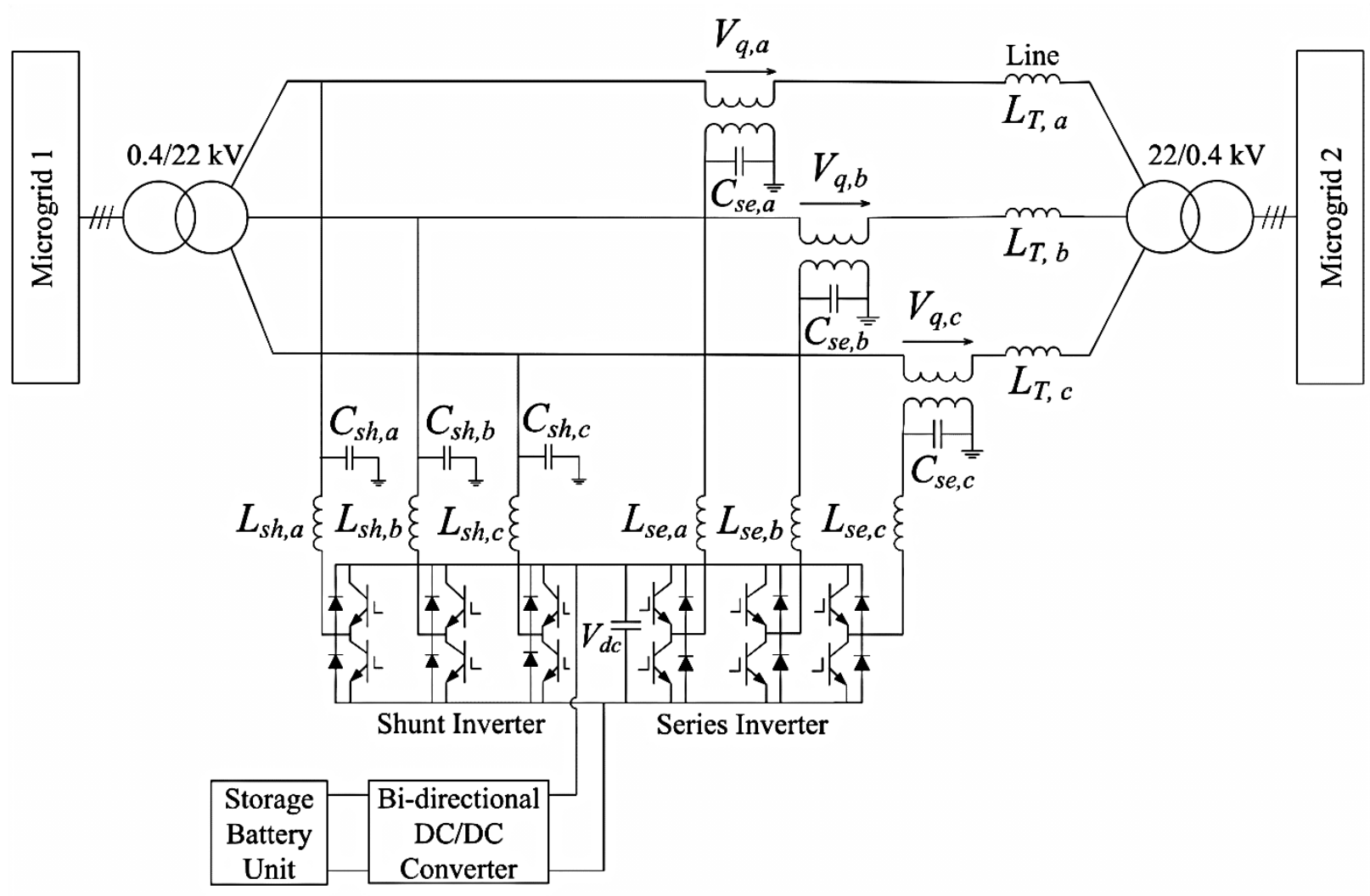

Figure 5-2: Configuration of the three-phase SSND.

\subsection{Modelling of Inverters in the Proposed System}

MPC is widely used in the control of inverters due to its many benefits. It can be applied to single-input single-output and multi-input multi-output systems [36]. Non-linearities and constraints can be easily incorporated, and modulation and coordinate transformations are not necessary in MPC algorithm [36]. Also, unlike other conventional control methods, MPC gives better transient response with respect to change in operating conditions [36] and ensures a more robust operation of the MMG system integrated with SSND. Moreover, as explained in Chapter 3, the proposed MPC algorithm is dependent only on the inverter parameters and is independent of the system parameters such as grid, line and load impedances. Thus, MPC can be used for multiple inverters in the MMG system for different system parameters. As DG inverters, series and shunt inverters work in different operating control modes, it is crucial for SSND to remain stable in the MMG system. Thus, to achieve optimal performance of both SSND and the interconnected microgrids, the proposed MPC algorithm is chosen in this research. The model of DG, series and shunt inverters is shown in 
Figure 5-3. The state-space model of the inverters is derived for CCM and VCM and then converted into augmented state-space model in the following sub-sections.

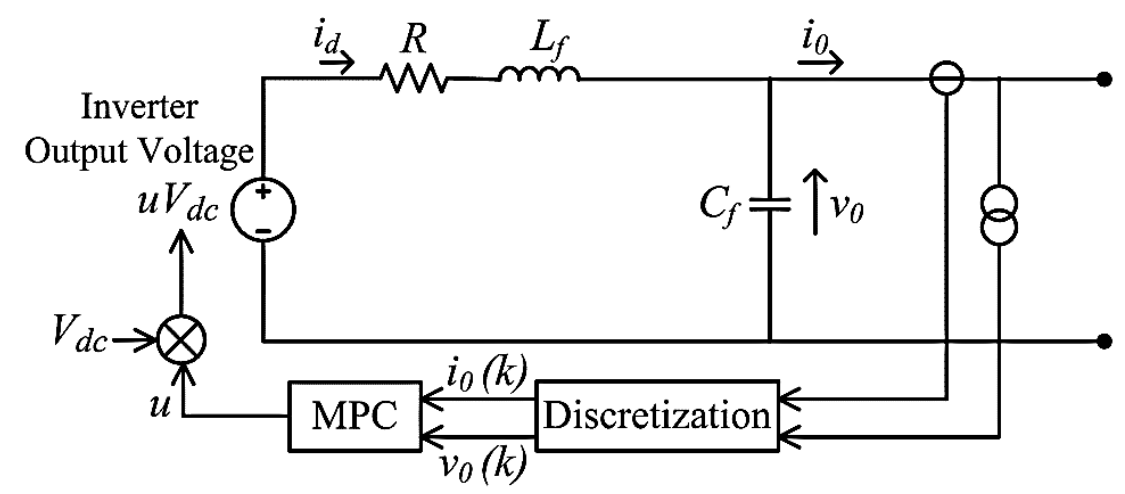

Figure 5-3: Single-phase inverter model.

\subsubsection{CCM of Inverter}

By applying Kirchhoff's voltage and current laws to the inverter model in Figure 5-3, the following equations are obtained:

$$
\begin{gathered}
u V_{d c}=i_{d} R+L_{f}\left(d i_{d} / d t\right)+v_{0} \\
i_{d}=C_{f}\left(d v_{0} / d t\right)+i_{0}
\end{gathered}
$$

where $R$ represents the internal loss of the inverter, $L_{f}$ and $C_{f}$ are the inductance and capacitance of the LC filter respectively, $i_{d}$ is the current flowing through $L_{f}, i_{0}$ is the inverter output current, $v_{0}$ is the inverter output voltage, and $u$ is the control input. Due to high sampling frequency, the following assumption can be made:

$$
d i_{0} / d t=0
$$

Due to this assumption, the state-space models need to be separately derived for CCM and VCM. By rearranging and simplifying (5.1)-(5.3) into a state-space form, the discrete statespace model for the inverter operating in CCM can be obtained as follows:

$$
\begin{gathered}
x_{1}(k+1)=A_{d} x_{1}(k)+B_{d} u(k) \\
y_{1}(k)=C_{d} x_{1}(k)
\end{gathered}
$$


where $x_{1}(k)=\left[\begin{array}{lll}i_{d}(k) & v_{0}(k) & v_{0}{ }^{\prime}(k)\end{array}\right]^{T}$ is the state vector, $u(k)$ is the control input vector, $y_{1}(k)=i_{O}(k)$ is the output vector, $v_{0}{ }^{\prime}=d v_{0} / d t$, and $A_{d}, B_{d}$ and $C_{d}$ are discretized coefficients obtained as follows with sampling time $T_{s}$.

$$
A_{d}=\left[\begin{array}{ccc}
1-\left(R T_{s} / L_{f}\right) & -T_{s} / L_{f} & 0 \\
0 & 1 & T_{s} \\
-T_{s} R /\left(L_{f} C_{f}\right) & -T_{s} /\left(L_{f} C_{f}\right) & 1
\end{array}\right], B_{d}=\left[\begin{array}{c}
V_{d c} T_{s} / L_{f} \\
0 \\
T_{s} /\left(L_{f} C_{f}\right)
\end{array}\right], C_{d}=\left[\begin{array}{lll}
1 & 0 & -C_{f}
\end{array}\right]
$$

In order to derive the control algorithm for the inverters, the discrete state-space model is converted into augmented state-space model by defining the incremental variables in the following format:

$$
\Delta \alpha(k)=\alpha(k)-\alpha(k-1)
$$

where $\alpha$ represents each variable in the inverter model such as $v_{0}, i_{0}, i_{d}$ and $u$ as shown in Fig. 5-3. By defining these incremental variables, the augmented state-space model of the inverter in CCM is derived as follows:

$$
\begin{gathered}
X_{e}(k+1)=A_{e} X_{e}(k)+B_{e} U_{e}(k) \\
Y_{e}(k)=C_{e} X_{e}(k)
\end{gathered}
$$

where $X_{e}(k)=\left[\begin{array}{llll}\Delta i_{d}(k) & \Delta v_{0}(k) & \Delta v_{0}{ }^{\prime}(k) & i_{0}(k)\end{array}\right]^{T}$ is the state vector, $U_{e}(k)=\Delta u(k)$ is the control input vector, $Y_{e}(k)=i_{0}(k)$ is the output vector, and the corresponding coefficients $A_{e}$, $B_{e}$ and $C_{e}$ are as follows.

$$
\begin{gathered}
A_{e}=\left[\begin{array}{cccc}
1-\left(R T_{s} / L_{f}\right) & -T_{s} / L_{f} & 0 & 0 \\
0 & 1 & T_{s} & 0 \\
-T_{s} R /\left(L_{f} C_{f}\right) & -T_{s} /\left(L_{f} C_{f}\right) & 1 & 0 \\
1 & 0 & -C_{f} & 1
\end{array}\right], C_{e}=\left[\begin{array}{llll}
0 & 0 & 0 & 1
\end{array}\right] \\
B_{e}=\left[\begin{array}{llll}
V_{d c} T_{s} / L_{f} & 0 & T_{s} / L_{f} C_{f} & \left(T_{s} / L_{f}\right)\left(V_{d c}-1\right)
\end{array}\right]^{T}
\end{gathered}
$$


Using this augmented state-space model, the output current and power of the DG and shunt inverters can be regulated at the desired reference values. Basic concept of an augmented state-space model is given in [110].

\subsubsection{VCM of Inverter}

DG inverter in a given microgrid in the MMG system shifts its mode of operation from CCM to VCM when that particular microgrid is islanded from its respective distribution grid. Also, the series inverter of the SSND is always operated in VCM to control the magnitude and angle of the injected voltage. From (5.1) and (5.2), the discrete state-space model for the inverter operating in VCM can be obtained as follows:

$$
\begin{gathered}
x_{2}(k+1)=A_{f} x_{2}(k)+B_{f} u(k)+B_{g} W(k) \\
y_{2}(k)=C_{h} x_{2}(k)
\end{gathered}
$$

where $x_{2}(k)=\left[\begin{array}{ll}i_{d}(k) & v_{0}(k)\end{array}\right]^{T}$ is the state vector, $u(k)$ is the control input vector, $W(k)=$ $i_{0}(k)$ is the disturbance vector, and $y_{2}(k)=v_{0}(k)$ is the output vector. $A_{f}, B_{f}, B_{g}$ and $C_{h}$ are discretized coefficients obtained as follows:

$$
A_{f}=\left[\begin{array}{cc}
1-\left(R T_{s} / L_{f}\right) & -T_{s} / L_{f} \\
-T_{s} / \mathrm{C}_{f} & 1
\end{array}\right], B_{f}=\left[\begin{array}{c}
V_{d c} T_{s} / L_{f} \\
0
\end{array}\right], C_{h}=\left[\begin{array}{ll}
0 & 1
\end{array}\right], B_{g}=\left[\begin{array}{ll}
0 & -T_{s} / \mathrm{C}_{f}
\end{array}\right]^{T}
$$

From the discrete state-space model, the augmented state-space model of the inverter in VCM is obtained as follows:

$$
\begin{gathered}
X_{g}(k+1)=A_{g} X_{g}(k)+B_{h} U_{g}(k)+B_{j} W_{g}(k) \\
Y_{g}(k)=C_{g} X_{g}(k)
\end{gathered}
$$

where $X_{g}(k)=\left[\begin{array}{llll}\Delta i_{d}(k) & \Delta v_{0}(k) & v_{0}(k)\end{array}\right]^{T}$ is the state vector, $U_{g}(k)=\Delta u(k)$ is the control input vector, $W_{g}(k)=\Delta i_{0}(k)$ is the disturbance vector and $Y_{g}(k)=v_{0}(k)$ is the output vector. 


$$
\begin{gathered}
A_{g}=\left[\begin{array}{ccc}
1-\left(R T_{s} / L_{f}\right) & -T_{s} / L_{f} & 0 \\
T_{s} / C_{f} & 1 & 0 \\
T_{s} / C_{f} & 1 & 1
\end{array}\right], B_{h}=\left[\begin{array}{c}
V_{d c} T_{s} / L_{f} \\
0 \\
0
\end{array}\right], \\
B_{j}=\left[\begin{array}{lll}
0 & -T_{s} / C_{f} & -T_{s} / C_{f}
\end{array}\right]^{T}, C_{g}=\left[\begin{array}{lll}
0 & 0 & 1
\end{array}\right]
\end{gathered}
$$

In order to control the augmented state-space models in CCM and VCM, quadratic programming is used to minimize the following objective function [110]:

$$
J=\left(R_{S}-Y\right)^{T}\left(R_{S}-Y\right)
$$

subject to the input and input increment constraints as follows:

$$
\begin{gathered}
-1 \leq u(k) \leq 1 \\
-1 \leq \Delta u(k) \leq 1
\end{gathered}
$$

where $R_{S}$ is the set-point matrix, $Y$ is the output of either the augmented model in CCM or VCM (i.e., $Y$ is equal to $Y_{e}$ in (5.8) or $Y_{g}$ in (5.12)). This objective function is used to ensure that the output of the augmented model tracks the reference with minimum error. Using the augmented model, objective function and constraints, the MPC algorithm thus generates the optimal control signal $u$. Then this optimal control signal is applied to the inverter as shown in Figure 5-3.

\subsection{Analytical Background}

The power quality and power flow control functions of SSND are analyzed in the following sub-sections:

\subsubsection{Harmonic Current Compensation}

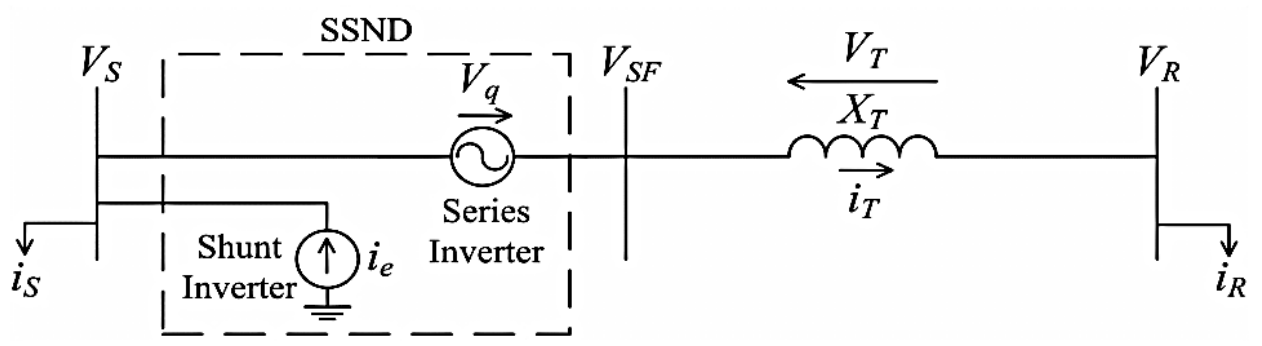

Figure 5-4: Principle of harmonic current compensation. 
The principle of harmonic current compensation is studied considering a section of the SSND-controlled line as shown in Figure 5-4. Let the total load currents in microgrids 1 and 2 be represented by the phasor quantities $i_{S}$ and $i_{R}$ respectively as follows:

$$
i_{S}=i_{S 1}+\sum_{h=2}^{k} i_{S h} ; i_{R}=i_{R 1}+\sum_{h=2}^{k} i_{R h}
$$

where the harmonic load current components in microgrids 1 and 2 are represented by the phasor quantities $\sum_{h=2}^{k} i_{S h}$ and $\sum_{h=2}^{k} i_{R h}$ respectively, $i_{S 1}$ and $i_{R 1}$ are the fundamental current components in load 1 and load 2 currents respectively, $h$ is the harmonic order and $k$ is the maximum order of harmonics in load 1 and load 2 currents. These harmonic load current components are extracted from their corresponding total load currents using a simple Fast Fourier Transform (FFT) method [116]. To compensate for the total harmonic load currents in the overall system, the series inverter and shunt inverter of SSND are required to inject the desired reference voltage and current respectively. To achieve harmonic current compensation in both the microgrids, the shunt inverter needs to inject the following reference harmonic current $i_{e}$, which is the sum of harmonic load currents in the microgrids.

$$
i_{e}=\sum_{h=2}^{k} i_{S h}+\sum_{h=2}^{k} i_{R h}
$$

where $i_{e}$ is a phasor quantity containing only harmonic components. The reference voltage to be injected by the series inverter to achieve harmonic current compensation in microgrid 2 is derived as follows:

Using Kirchhoff's voltage law, following equation is obtained from Figure 5-4.

$$
V_{S}+V_{q}=V_{S F}=V_{R}+V_{T}=V_{R}+i_{T}\left(j X_{T}\right)
$$

where $V_{S}, V_{q}, V_{S F}, V_{T}, V_{R}$ and $i_{T}$ represent phasor quantities. $V_{T}$ is the voltage drop across the line reactance $X_{T}$. $V_{S}$ and $V_{R}$ are assumed to be purely sinusoidal and consist of only 
fundamental components, and $V_{q}$ and $i_{T}$ consist of fundamental and harmonic components as follows:

$$
\begin{gathered}
V_{S}=V_{S 1} ; V_{q}=V_{q 1}+\sum_{h=2}^{k} V_{q h} \\
V_{R}=V_{R 1} ; i_{T}=i_{T 1}+\sum_{h=2}^{k} i_{T h}
\end{gathered}
$$

where subscripts ' 1 ' and ' $h$ ' represent fundamental and harmonic components respectively, $i_{T 1}$ and $\sum_{h=2}^{k} i_{T h}$ represent the fundamental and harmonic components in the line current $i_{T}$. In order for SSND to compensate for the harmonic currents in microgrid 2, the following condition should be satisfied:

$$
\sum_{h=2}^{k} i_{T h}=\sum_{h=2}^{k} i_{R h}
$$

Substituting the values of $V_{S}, V_{q}, V_{R}$ and $i_{T}$ in (5.18) and comparing the fundamental and harmonic components, the required injected voltage to facilitate the transfer of harmonic current in (5.21) through the line from SSND to microgrid 2 is obtained as follows:

$$
\sum_{h=2}^{k} V_{q h}=\sum_{h=2}^{k} i_{R h}\left(j h L_{T} \omega\right)
$$

Thus SSND achieves harmonic current compensation in both the microgrids in the MMG system by simultaneously controlling its shunt inverter to inject the required current given by (5.17) and controlling its series inverter to inject the required voltage given by (5.22), and by using the principle of MPC shown in Figure 5-3.

\subsubsection{Isolation of Voltage Disturbances}

SSND can be used to isolate one microgrid from voltage disturbances such as voltage distortion, voltage sag and voltage unbalance occurring in the adjacent microgrid in the MMG system. The voltage distortions can occur in load bus voltages of the microgrids due to voltage distortions in distribution grid voltage. Voltage sags in load bus voltages can occur due to balanced and unbalanced faults at different locations in the overall system such as load 
buses, distribution grid buses and along the line interconnecting the microgrids. SSND can be used to isolate microgrid 2 from voltage disturbances in microgrid 1 or distribution grid 1 . If distribution grid 1 contains voltage harmonics and voltage unbalance, and experiences voltage sag for a short period, then the voltage to be injected by the series inverter of SSND to achieve voltage disturbance isolation and obtain a sinusoidal load bus voltage at microgrid 2 is given as follows:

$$
V_{q}=V_{q h}+V_{q u}+V_{q s}
$$

where phasors $V_{q h}$ and $V_{q u}$ represent the harmonic components and unbalance components respectively in distribution grid 1 voltage, and vector $V_{q s}$ represents the missing voltage component in distribution grid 1 voltage due to voltage sag. Similarly, SSND can also be used to isolate microgrid 1 from voltage disturbances in microgrid 2 or distribution grid 2. Thus SSND improves the voltage quality of the MMG system by completely isolating one microgrid from the voltage disturbances originating in the other microgrid.

\subsubsection{Real and Reactive Power Flow Control}

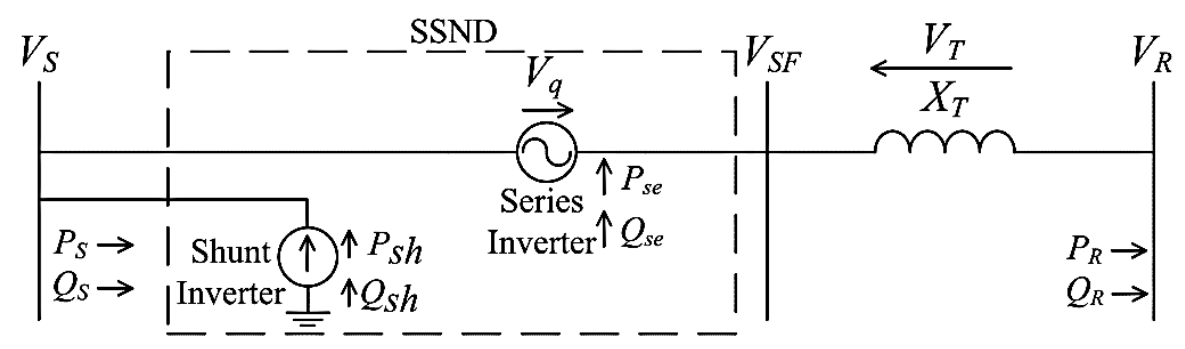

Figure 5-5: Principle of real and reactive power control.

In Figure 5-5, let $V_{S}=V \angle 0^{\circ}, V_{R}=V \angle-\delta, V_{q}=V_{s e} \angle \theta$. The power flow equations are derived as follows:

$$
\begin{gathered}
P_{S}=\left[V^{2} \sin \delta+V V_{s e} \sin \theta\right] / X_{T} \\
P_{s e}=\left[V V_{s e} \sin (\delta+\theta)-V V_{s e} \sin \theta\right] / X_{T} \\
P_{R}=\left[V^{2} \sin \delta+V V_{s e} \sin (\delta+\theta)\right] / X_{T} \\
Q_{S}=\left[V^{2}(1-\cos \delta)+V V_{s e} \cos \theta\right] / X_{T}
\end{gathered}
$$




$$
\begin{gathered}
Q_{s e}=\left[V V_{s e} \cos \theta-V V_{s e} \cos (\delta+\theta)+V_{s e}^{2}\right] / X_{T} \\
Q_{R}=\left[V^{2} \cos \delta+V V_{s e} \cos (\delta+\theta)-V^{2}\right] / X_{T} \\
\text { Also, }\left(P_{R}-P_{R 0}\right)^{2}+\left(Q_{R}-Q_{R 0}\right)^{2}=\left(V V_{s e} / X_{T}\right)^{2}
\end{gathered}
$$

where $P_{R 0}=V^{2} \sin \delta / X_{T}$ and $Q_{R 0}=V^{2}(1-\cos \delta) / X_{T}$ represent the receiving end real and reactive power respectively without SSND.

The phasor diagram of the system voltages according to Figure 5-5 and equation (5.18) is given in Figure 5-6. Equation (5.30) represents a circular control region of attainable receiving end real power $P_{R}$ and reactive power $Q_{R}$ with center 'c' defined by the coordinates $\left(P_{R 0}, Q_{R 0}\right)$ and radius ' $\mathrm{r}$ ' equal to $V V_{s e} / X_{T}$ obtained with a SSND-controlled line, which is shown for $\delta=0^{\circ}$ in Figure 5-7. The real and reactive power flow control functions of the SSND are explained with reference to different values of $\delta$ and $\theta$ as follows:

\subsubsection{Reactive Power Compensation with SSND during Grid-Connected Steady-State Operation of the MMG System}

At $\delta=\theta=0^{\circ}$, the power flow equations (5.24) - (5.29) are transformed as follows:

$$
\begin{gathered}
P_{S}=P_{s e}=P_{R}=0 \\
Q_{S}=Q_{R}=V V_{s e} / X_{T} \\
Q_{s e}=V_{s e}^{2} / X_{T}
\end{gathered}
$$

Let the total load reactive power demand in microgrids 1 and 2 be given by

$$
Q_{L 1}=Q_{l 1}+Q_{n 1} ; Q_{L 2}=Q_{l 2}+Q_{n 2}
$$

To supply the desired reactive powers $Q_{L 1}$ and $Q_{L 2}$ to loads 1 and 2 respectively, the reactive power to be injected by the shunt inverter of the SSND is given by

$$
Q_{s h}=Q_{L 1}+Q_{L 2}=Q_{L 1}+Q_{R} ;\left(\text { where } Q_{L 2}=Q_{R}\right)
$$

To obtain the desired reactive power $Q_{R}$ at the receiving end of the interconnecting line, the voltage to be injected by the series inverter of the SSND based on equation (5.32) is given by 


$$
V_{s e}=\left[Q_{R} X_{T} / V\right] \angle 0^{\circ}=\left[Q_{L 2} X_{T} / V\right] \angle 0^{\circ}
$$

Therefore SSND achieves entire reactive power compensation in both the microgrids during steady-state operation of the proposed system by simultaneously controlling its shunt inverter to inject the required reactive power given by (5.35) and controlling its series inverter to inject the required voltage given by (5.36) using the principle of MPC shown in Figure 5-3. Also, in this case the real power injected by the series inverter is $0 \mathrm{~W}$ as given in (5.31), which thus eliminates any real power injection by the series inverter interfaced storage battery unit of the SSND. In addition, the real power flow through the line is maintained at 0 $\mathrm{W}$ in this case as given in (5.31). The operating point of the SSND at $\delta=\theta=0^{\circ}$ is indicated by point ' $A$ ' on the circular control region shown in Figure 5-7.

\subsubsection{Combined Real Power Support and Reactive Power Compensation with SSND during Islanding of a Microgrid in the MMG System}

At $\delta=0^{\circ}, \theta \neq 0^{\circ}$, the power flow equations (5.24) - (5.29) are changed as follows:

$$
\begin{gathered}
P_{S}=P_{R}=\left[V V_{s e} \sin \theta / X_{T}\right] \\
Q_{S}=Q_{R}=\left[V V_{s e} \cos \theta / X_{T}\right] \\
P_{s e}=0 \\
Q_{s e}=V_{s e}^{2} / X_{T}
\end{gathered}
$$

If the desired receiving end real power $P_{R}$ and reactive power $Q_{R}$ are to be supplied to microgrid 2, which is islanded from distribution grid 2, then the required phase angle and magnitude of the voltage to be injected by the series inverter of the SSND is obtained from (5.37) and (5.38) as follows:

$$
\theta=\tan ^{-1}\left(P_{R} / Q_{R}\right)=\tan ^{-1}\left(P_{R} / Q_{L 2}\right)
$$

Substituting the value of $\theta$ in (5.37), the required injection voltage $V_{s e}$ is given as 


$$
V_{s e}=P_{R} X_{T} / V \sin \theta=P_{R} X_{T} /\left[V \sin \left(\tan ^{-1}\left(P_{R} / Q_{L 2}\right)\right)\right]
$$

The receiving end real power $P_{R}$ to be externally supplied to the islanded microgrid 2 is shared among the shunt inverter of SSND and DG unit 1 of microgrid 1 in a predetermined ratio $\alpha: \beta$, based on the maximum power ratings of DG unit 1 and storage battery unit of the SSND. Thus the real power to be injected by the shunt inverter of the SSND in this case is given by

$$
P_{s h}=\alpha P_{R}
$$

The reactive power to be injected by the shunt inverter of the SSND in this case is also the same as given in (5.35). In addition, during the voltage injection, the reactive power injected by the series inverter given by (5.40) compensates for the reactive power loss in the line. The operating point ' $\mathrm{B}$ ' of the SSND at $\delta=0^{\circ}, \theta \neq 0^{\circ}$ is denoted by point ' $\mathrm{B}$ ' in the circular control region shown in Figure 5-7. Thus, during islanding of a microgrid from its respective distribution grid, SSND is capable to provide real power support to meet power shortage in the islanded microgrid and is also simultaneously capable to provide entire reactive power to the loads in both grid-connected and islanded microgrids.

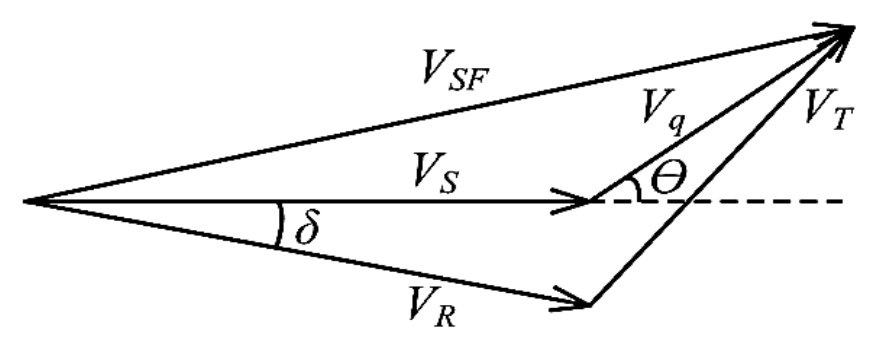

Figure 5-6: Phasor diagram of the system voltages.

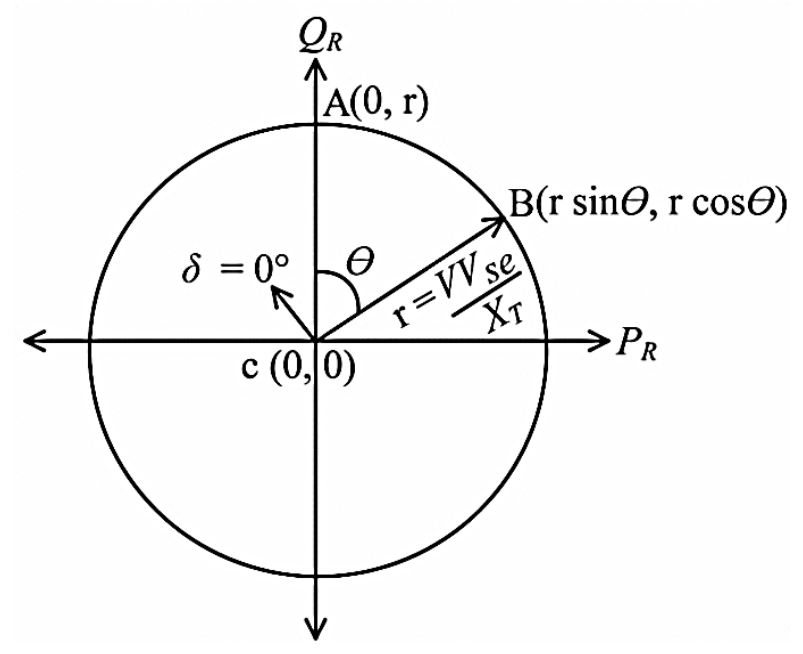

Figure 5-7: Control region of the attainable receiving end real power $P_{R}$ and reactive power $Q_{R}$ with a SSND-controlled line at $\delta=0^{\circ}$ (where c is the center and $\mathrm{r}$ is the radius). 


\subsection{Simulation Studies}

Simulation studies are conducted using MATLAB/Simulink to verify the performance of the SSND in the proposed MMG system. Linear loads 1 and 2 are three-phase RL loads with a real and reactive power demand of $5 \mathrm{MW}$ and $2 \mathrm{MVAr}$ respectively. Non-linear loads 1 and 2 are three-phase dimmer loads with fundamental real and reactive power demand of $5 \mathrm{MW}$ and 2 MVAr respectively. The two dimmer loads have same harmonic current spectra, which are determined through the experimental results obtained from several light dimmers in [96]. The parameters of the proposed system are shown in Table 5-1.

Table 5-1: Parameters of the proposed system

\begin{tabular}{l|l}
\hline \multicolumn{1}{c|}{ Parameter } & \multicolumn{1}{c}{ Value } \\
\hline Inverter input voltage & $V_{d c}=800 \mathrm{~V}$ \\
\hline Loss resistance of inverter & $R=1 \mu \Omega$ \\
\hline LC filter of inverter & $L_{f}=1 \mu \mathrm{H}, C_{f}=0.1 \mathrm{~F}$ \\
\hline Supply voltage of distribution grid & $V_{g}=22000 / \sqrt{3} \mathrm{Vrms}$ (phase) \\
\hline Line inductance & $L_{T}=2 \mathrm{mH}$ \\
\hline Leakage inductance of transformer & $L_{m}=0.1 \mathrm{mH}$ \\
\hline
\end{tabular}

Details of different component ratings:

\begin{tabular}{l|l}
\hline a) DG unit 1 & $8 \mathrm{MW}(\max )$ \\
\hline b) DG unit 2 & $6 \mathrm{MW}(\max )$ \\
\hline c) Storage battery unit of SSND & $2 \mathrm{MW}(\max )$ \\
\hline d) Transformer & $10 \mathrm{MVA}, 22 / 0.4 \mathrm{kV}$ \\
\hline
\end{tabular}

\subsubsection{Test Case 1 - Harmonic Current Compensation}

In the first test case, the capability of the SSND to perform harmonic current compensation in the MMG system is demonstrated. To compensate for load 2 harmonic currents given by (5.21), the series inverter of the SSND is controlled to inject the harmonic voltage given in (5.22), which is shown in Figure 5-8. The shunt inverter of the SSND is controlled to inject the harmonic current given by (5.17), which is shown in Figure 5-9. Due to the voltage and current injections by the series and shunt inverters respectively, the line current as seen in Figure 5-10 is obtained. The distorted harmonic current in total load 1 (total load 2) is shown in Figure 5-11. With the help of series and shunt inverters as described earlier, SSND 
effectively compensates the harmonic load currents. The compensated distribution grid 1 (distribution grid 2) current thus obtained is shown in Figure 5-12. The harmonic components in distribution grid 1 (distribution grid 2) current are suppressed as observed in the harmonic spectra, shown in Figure 5-13. The total harmonic distortion (THD) value of total load 1 (total load 2) current is $12.16 \%$ whereas the THD value of distribution grid 1 (distribution grid 2) current is $2.4 \%$ only, which is within the IEEE 519 allowable limit of $5 \%$ [82]. This demonstrates the capability of SSND in effective local and non-local harmonic current compensation in microgrid 1 and microgrid 2 respectively. The RMS values of harmonic currents and voltages in this test case are given in Table 5-2.

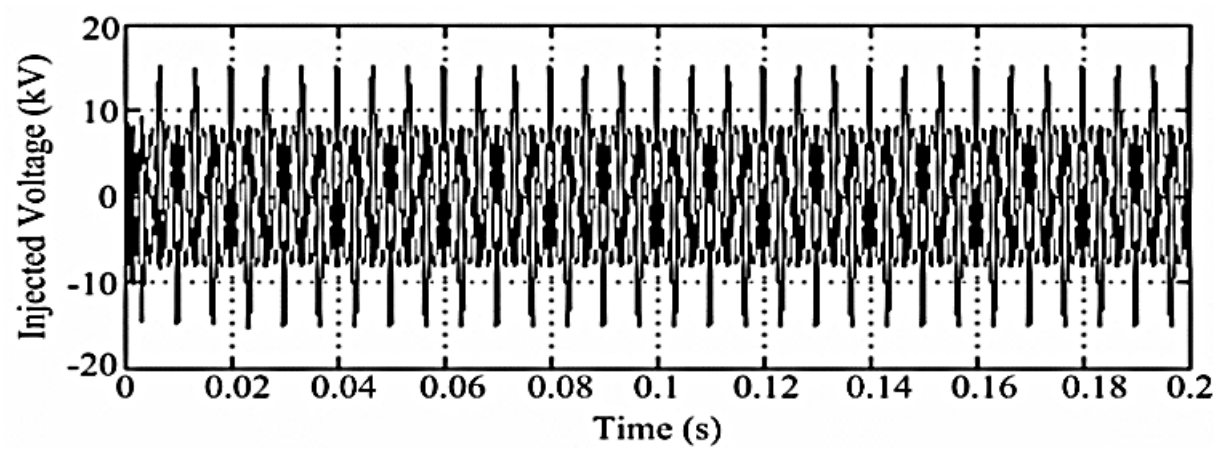

Figure 5-8: Injected voltage of the series inverter.

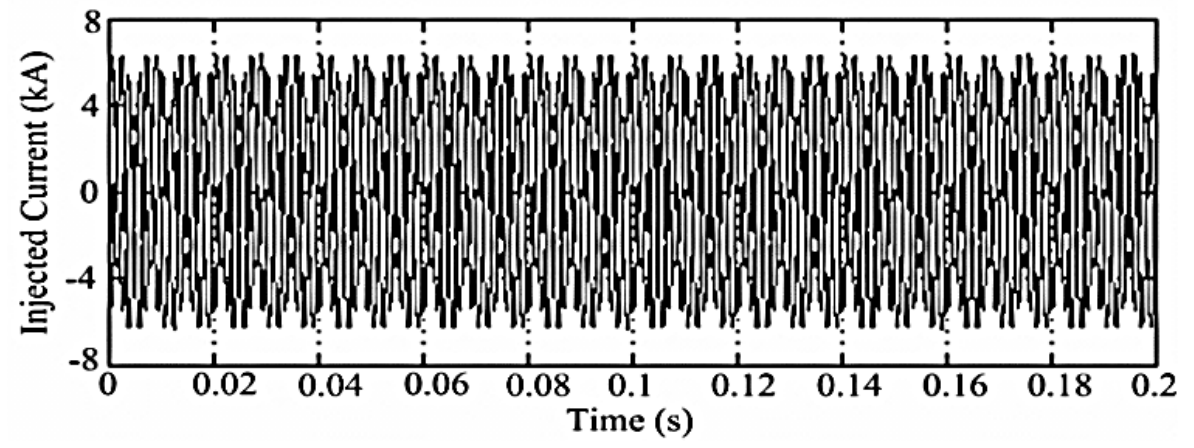

Figure 5-9: Injected current of the shunt inverter.

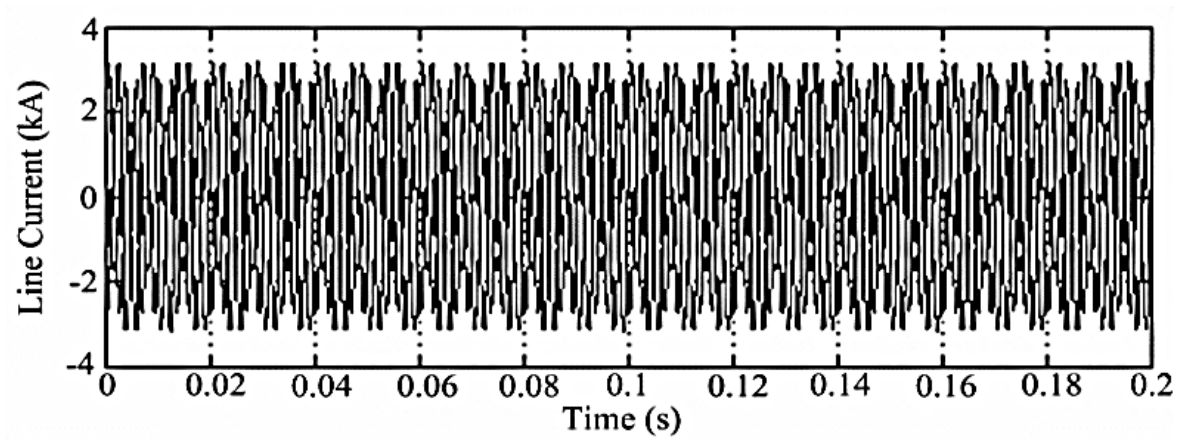

Figure 5-10: Line current. 


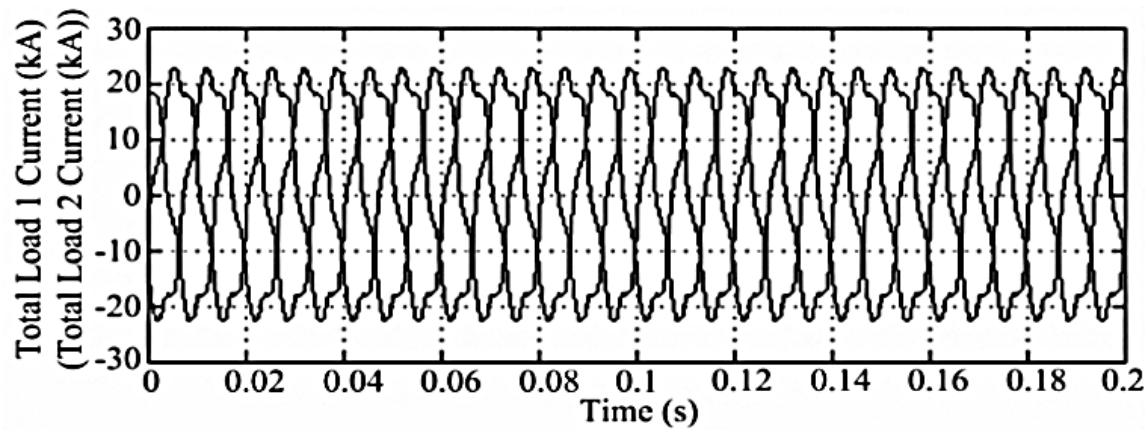

Figure 5-11: Distorted harmonic current in total load 1 (total load 2).

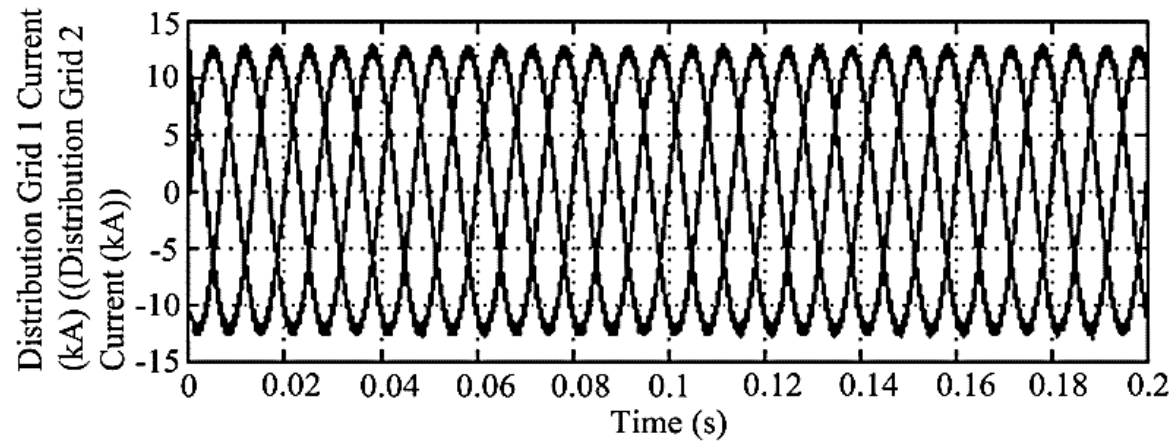

Figure 5-12: Distribution grid 1 current (Distribution grid 2 current).
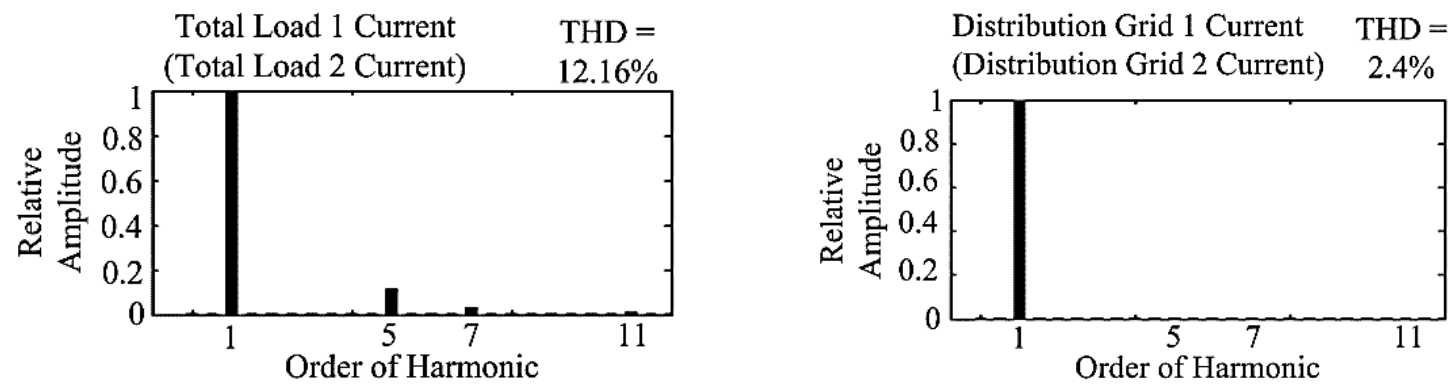

Figure 5-13: Harmonic spectra of total load and distribution grid currents.

Table 5-2: RMS values of harmonic currents and voltages

\begin{tabular}{l|l|l|l}
\hline $\begin{array}{l}\text { Harmonic } \\
\text { order }\end{array}$ & $\begin{array}{l}\text { Non-linear load 1 current } \\
(\mathrm{kA})(\text { Non-linear load 2 } \\
\text { current }(\mathrm{kA}))\end{array}$ & $\begin{array}{l}\text { Injected } \\
\text { current } \\
(\mathrm{kA})\end{array}$ & $\begin{array}{l}\text { Injected } \\
\text { voltage } \\
(\mathrm{kV})\end{array}$ \\
\hline 5 & $1.8 \angle 0^{\circ}$ & $3.6 \angle 0^{\circ}$ & $5.65 \angle 90^{\circ}$ \\
\hline 7 & $0.5 \angle 0^{\circ}$ & $1.0 \angle 0^{\circ}$ & $2.19 \angle 90^{\circ}$ \\
\hline 11 & $0.2 \angle 0^{\circ}$ & $0.4 \angle 0^{\circ}$ & $1.38 \angle 90^{\circ}$ \\
\hline
\end{tabular}




\subsubsection{Test Case 2 - Isolation of Voltage Harmonics/Unbalance and Fault-induced Voltage Sag}

To illustrate the use of the SSND in isolating microgrid 2 from the voltage distortions occurring in microgrid 1, load 1 is fed with a distorted and unbalanced distribution grid 1 voltage, whose harmonic spectra is shown in Table 5-3. The distorted load 1 bus voltage with and without SSND is shown in Figure 5-14. The distorted load 2 bus voltage without SSND is also shown in Figure 5-15. The THD values of load 2 bus voltages for phase A, phase B and phase $\mathrm{C}$ are $10.9 \%, 11.8 \%$ and $10.4 \%$ respectively. When SSND is connected in the line, the load 2 bus voltage is restored to a balanced sinusoidal voltage with the nominal phase value of $230 \mathrm{~V}$ (rms) and very low THD value of $0.2 \%$ as seen in Figure 5-16 because the series inverter of the SSND injects the required compensating voltage given in (5.23). To highlight the capability of the SSND in isolating microgrid 2 from fault-induced voltage sags in microgrid 1, a three-phase balanced fault and a single-phase unbalanced fault on phase A are simulated at load 1 bus for $0.05 \leq \mathrm{t} \leq 0.15 \mathrm{~s}$ and $0.2 \leq \mathrm{t} \leq 0.3 \mathrm{~s}$ respectively as shown in Figure 5-17. It can be seen that the load 1 bus voltage sags to $183.8 \mathrm{~V}$ (rms) in all three phases during three-phase fault and sags to $215.6 \mathrm{~V}$ (rms) in phase A during single-phase fault. Without SSND, it can be observed that the load 2 bus voltage sags to $189.3 \mathrm{~V}$ (rms) in all three phases and $216.8 \mathrm{~V}$ (rms) in phase A during three-phase fault and single-phase fault respectively as shown in Figure 5-18. Due to the voltage sag compensation capability of the SSND, load 2 bus voltage returns to its nominal value of $230 \mathrm{~V}$ (rms) as shown in Figure 519.

Table 5-3: Harmonic spectra of distribution grid 1 voltage

\begin{tabular}{l|l|l|l|l|l|l}
\hline \multirow{2}{*}{$\begin{array}{c}\text { Harmonic } \\
\text { order }\end{array}$} & \multicolumn{2}{|c|}{ Phase A } & \multicolumn{2}{c|}{ Phase B } & \multicolumn{2}{c}{ Phase C } \\
\cline { 2 - 7 } & RMS & Angle $\left(^{\circ}\right)$ & $\begin{array}{c}\text { RMS } \\
\text { value (V) }\end{array}$ & Angle $\left(^{\circ}\right)$ & $\begin{array}{c}\text { RMS } \\
\text { value (V) }\end{array}$ & Angle $\left(^{\circ}\right)$ \\
\hline 1 & 214 & 0 & 198 & -120 & 225 & 120 \\
\hline 5 & 20.5 & -160 & 20.5 & -760 & 20.5 & 440 \\
\hline 7 & 11.3 & -112 & 11.3 & -952 & 11.3 & 728 \\
\hline
\end{tabular}




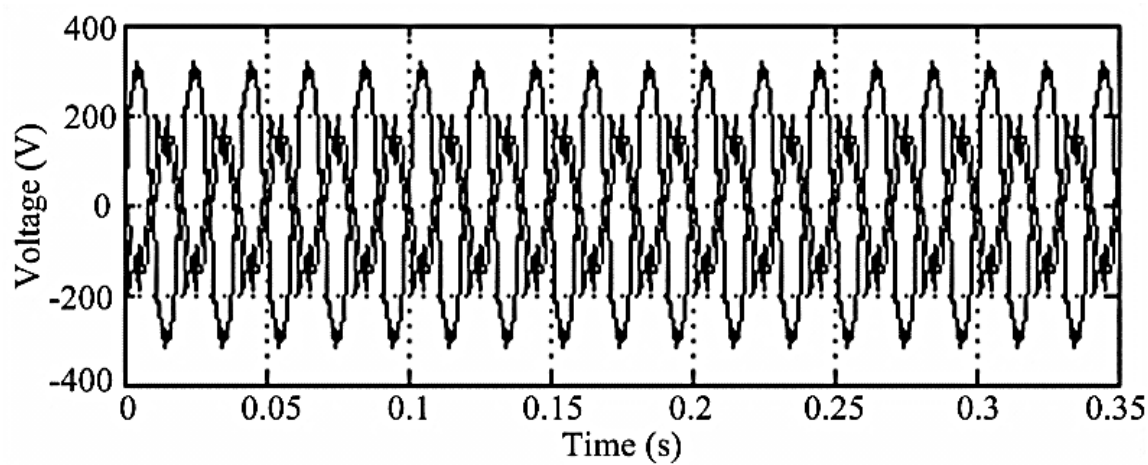

Figure 5-14: Load 1 bus voltage with and without SSND.

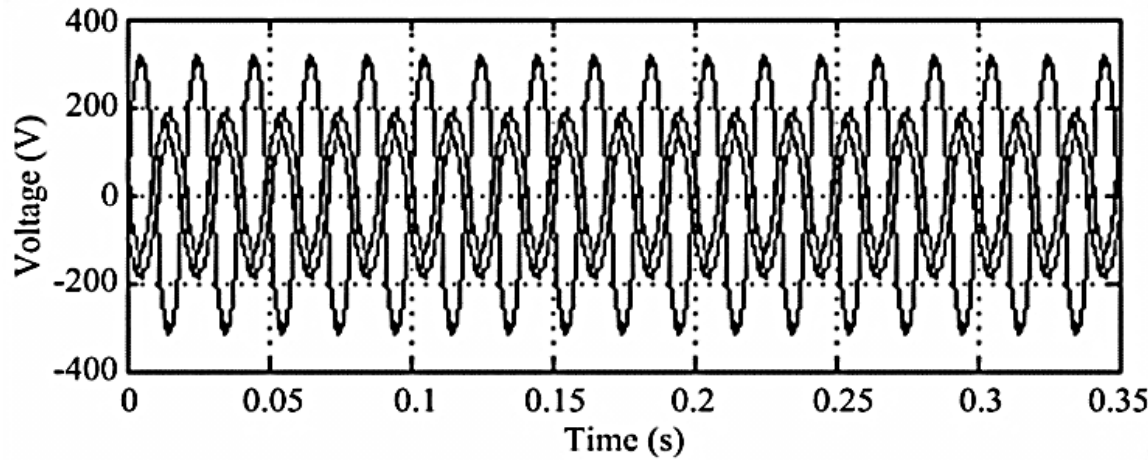

Figure 5-15: Load 2 bus voltage without SSND.

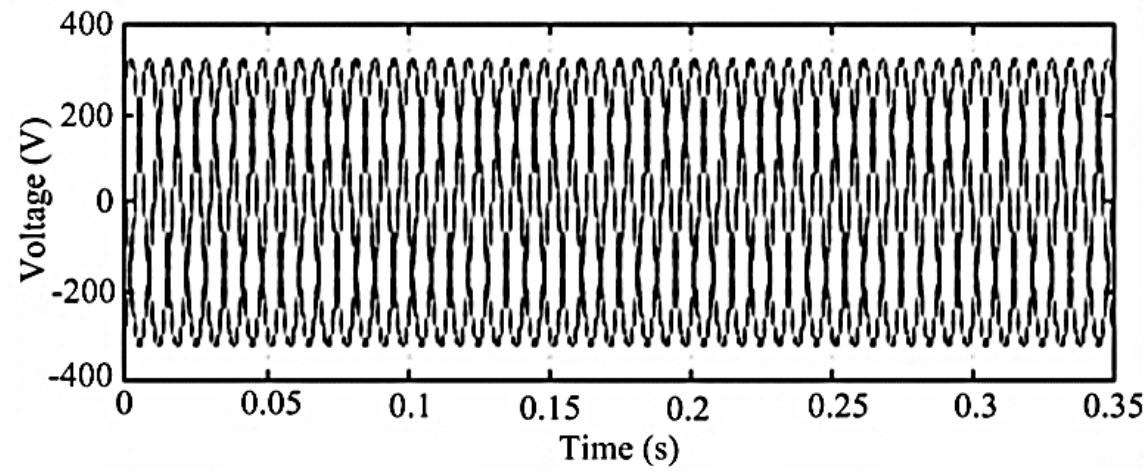

Figure 5-16: Load 2 bus voltage with SSND.

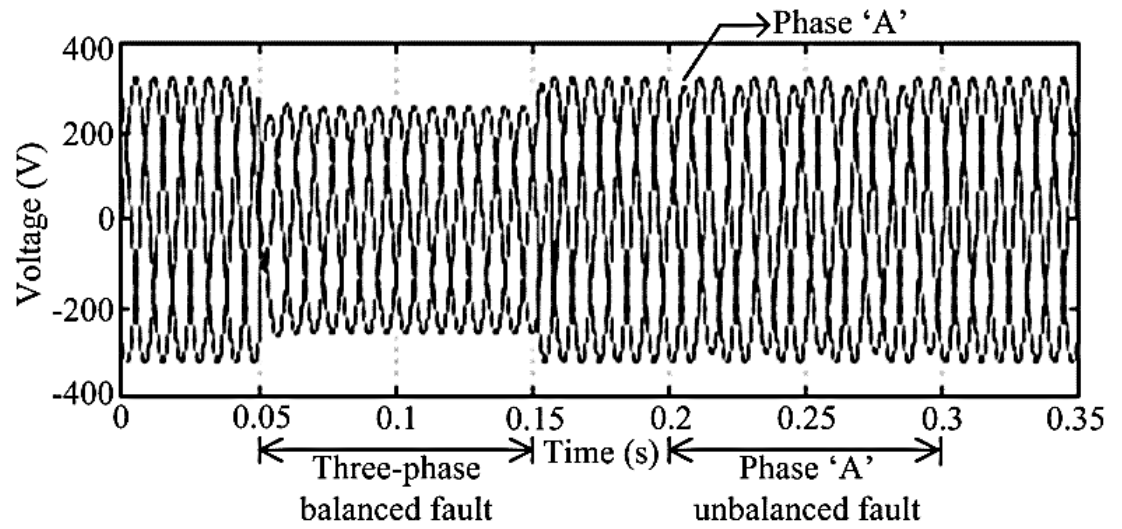

Figure 5-17: Load 1 bus voltage with and without SSND during voltage sags. 


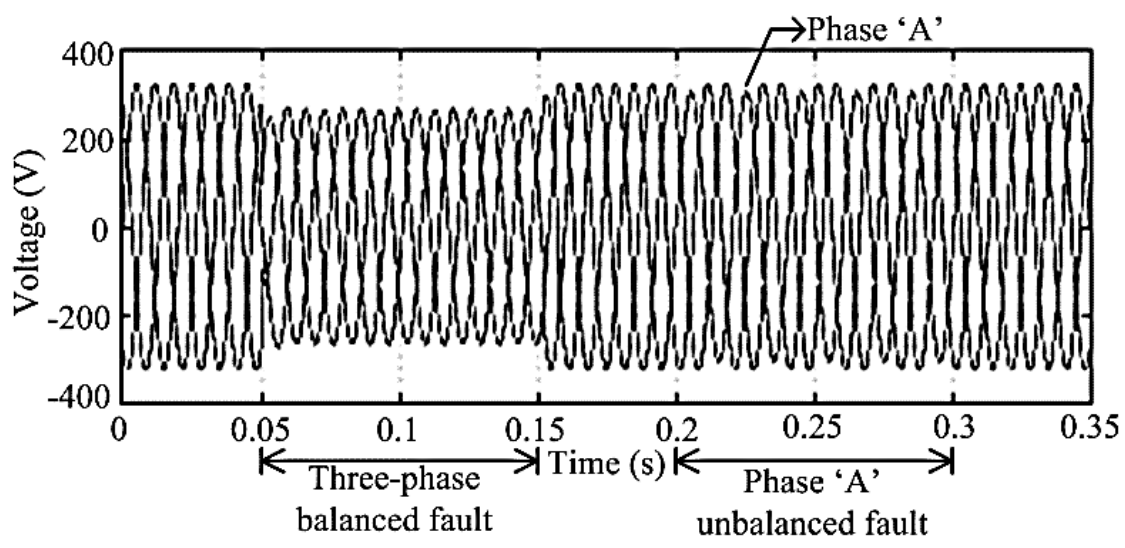

Figure 5-18: Load 2 bus voltage without SSND.

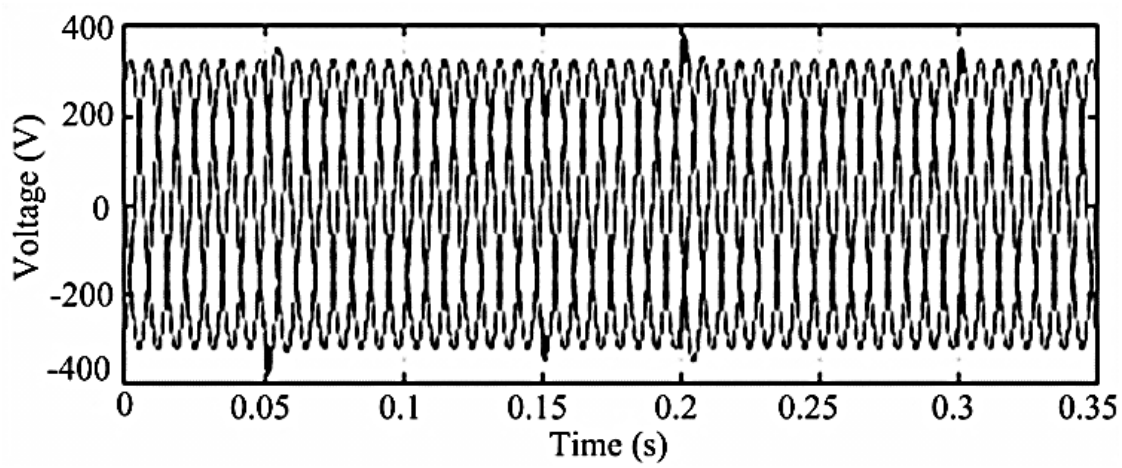

Figure 5-19: Load 2 bus voltage with SSND.

\subsubsection{Test Case 3 - SSND Functioning as a Real and Reactive Power Flow Controller}

The simulation begins with microgrids 1 and 2 operating in grid-connected mode for $0 \leq t \leq 4$ s during the steady-state operation of the proposed system. In this steady-state operation, SSND is required to provide the entire reactive power demand of loads 1 and 2 in microgrids 1 and 2 respectively. Thus the shunt inverter of the SSND is controlled to supply the entire reactive power demand of 8 MVAr as shown in Figure 5-20. In order to obtain the desired reactive power of $4 \mathrm{MVAr}$ at the receiving end of the line as shown in Figure 5-20, the series inverter of the SSND injects a voltage equal to $65.9 \angle 0^{\circ} \mathrm{V}(\mathrm{rms})$ according to (5.36) for $\mathrm{t}<4$ $\mathrm{s}$ as shown in Figure 5-21 and Figure 5-22. DG inverter 1 and DG inverter 2 supply $4 \mathrm{MW}$ each during steady-state operation as shown in Figure 5-23. At t $=4 \mathrm{~s}$, microgrid 2 is islanded from distribution grid 2 due to a fault in the upstream network. When microgrid 2 is islanded from distribution grid 2, it results in a loss of $6 \mathrm{MW}$ from distribution grid 2 and thus microgrid 2 will not be able to sustain its generation to meet the power demand of load 2. So microgrid 2 needs $3 \mathrm{MW}, 2 \mathrm{MW}$ and $1 \mathrm{MW}$ from DG inverter 1, DG inverter 2 and SSND respectively in accordance with the contract agreement among the microgrids and SSND. 
Thus, under this condition, the real power outputs of DG inverters 1 and 2 are controlled to increase from $4 \mathrm{MW}$ to $7 \mathrm{MW}$ and $4 \mathrm{MW}$ to $6 \mathrm{MW}$ respectively for $\mathrm{t}>4.1 \mathrm{~s}$ as shown in Figure 5-23. The real power output of the shunt inverter is controlled to increase from $0 \mathrm{MW}$ to $1 \mathrm{MW}$ for $\mathrm{t}>4.1 \mathrm{~s}$ as observed in Figure 5-23. A delay of $0.1 \mathrm{~s}$ is introduced between the islanding of microgrid 2 and the initiation of the inverters to cater for transient conditions in the system [18].

During islanding, in order to obtain the desired real and reactive power of $4 \mathrm{MW}$ and 4 MVAr at the receiving end of the line as shown in Figure 5-23 and Figure 5-20 respectively, a voltage equal to $92.9 \angle 45^{\circ} \mathrm{V}$ (rms) according to (5.41) and (5.42) is injected by the series inverter for $\mathrm{t}>4 \mathrm{~s}$ as shown in Figure 5-21 and Figure 5-22. From Figure 5-24, it can be observed that the distribution grids 1 and 2 deliver only real power and no reactive power during the steady-state operation, thus resulting in the achievement of unity power factor at the distribution grid side. This verifies that SSND achieves local and non-local reactive power compensation in microgrids 1 and 2 respectively. DG inverter 2 shifts its mode of operation from CCM in grid-connected mode to VCM in islanded mode to regulate the load 2 bus voltage at $230 \mathrm{~V}(\mathrm{rms})$ for $\mathrm{t}>4 \mathrm{~s}$ as shown in Figure 5-25. During the transition of microgrid 2 from grid-connected to islanded mode, there is a power imbalance in the overall system which causes the system frequency to drop to $49.8 \mathrm{~Hz}$ at $\mathrm{t}=4.4 \mathrm{~s}$ as shown in Figure 5-26. Since the DG inverters and shunt inverter supply the required powers to rectify this power imbalance problem, the system frequency is gradually restored to its nominal value of $50 \mathrm{~Hz}$ for $\mathrm{t} \geq 7 \mathrm{~s}$.

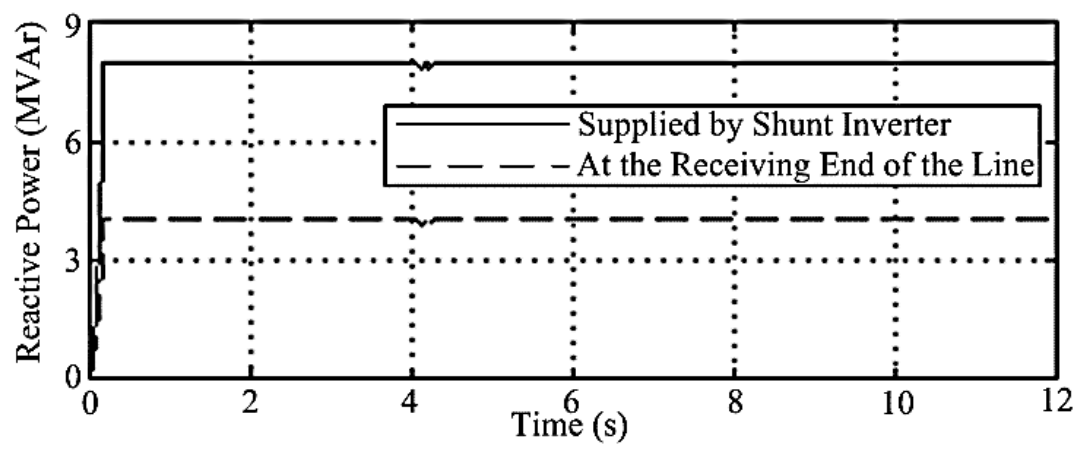

Figure 5-20: Reactive power supplied by the shunt inverter and reactive power at the receiving end of the line. 


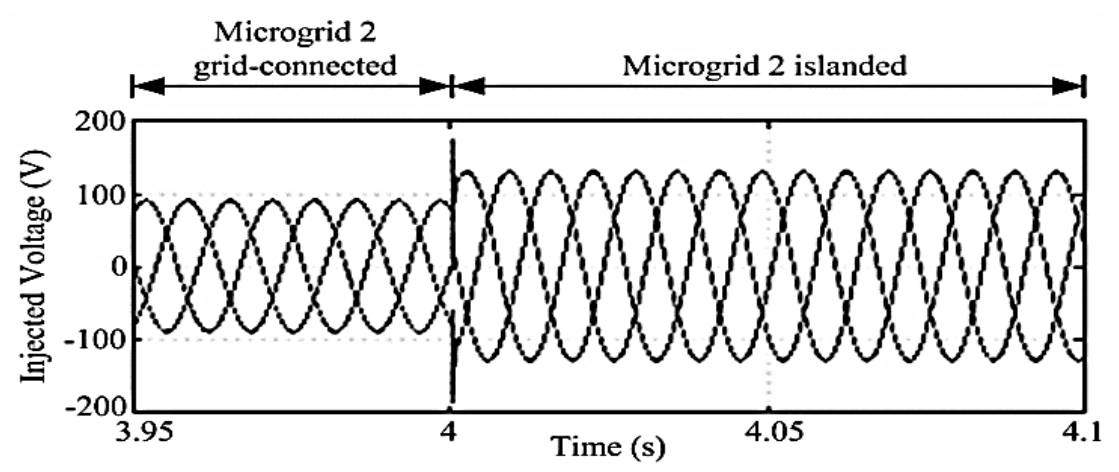

Figure 5-21: Injected voltage of the series inverter for $3.95 \leq \mathrm{t} \leq 4.1 \mathrm{~s}$.

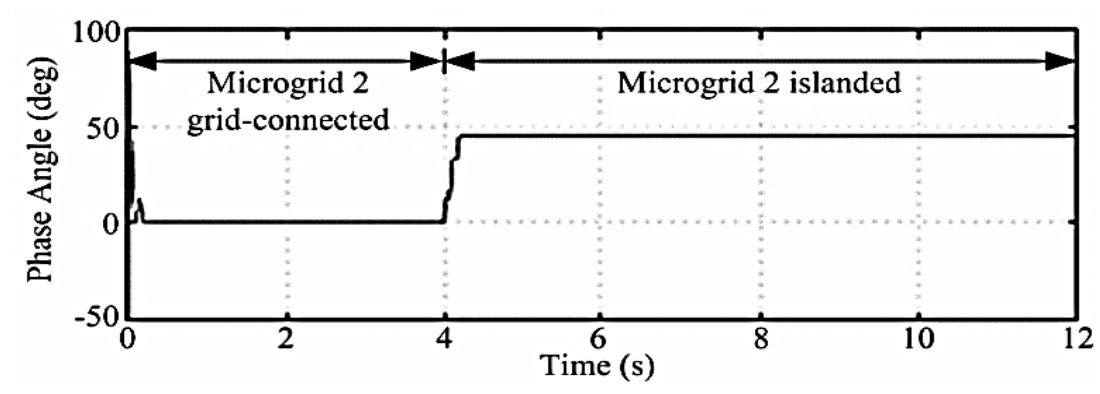

Figure 5-22: Phase angle of injected voltage.

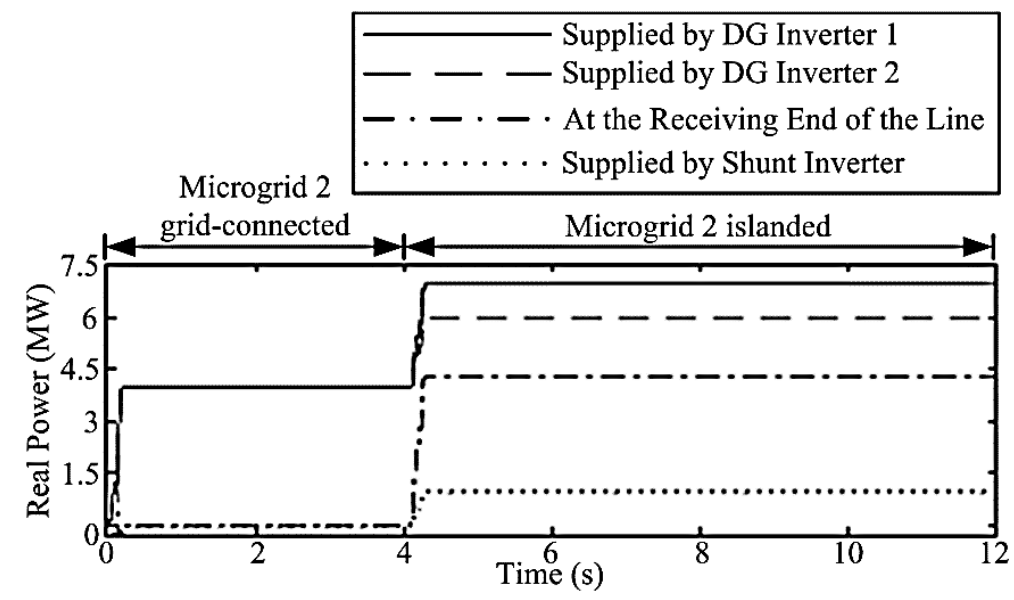

Figure 5-23: Real powers supplied by DG inverters 1 and 2 and shunt inverter, and real power at the receiving end of the line.

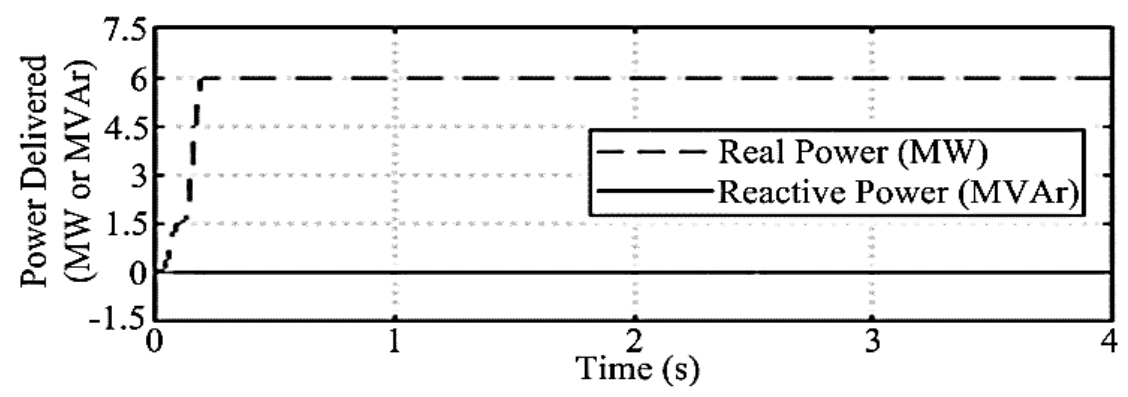

Figure 5-24: Real and reactive power delivered by distribution grids 1 and 2 during steadystate operation of the MMG system. 


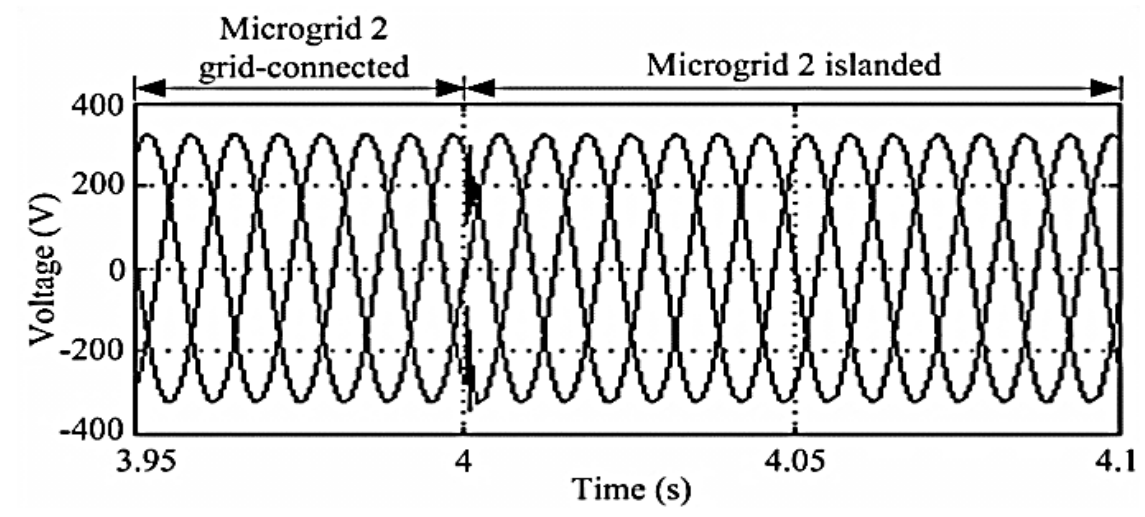

Figure 5-25: Three-phase load 2 bus voltage for $3.95 \leq \mathrm{t} \leq 4.1 \mathrm{~s}$.

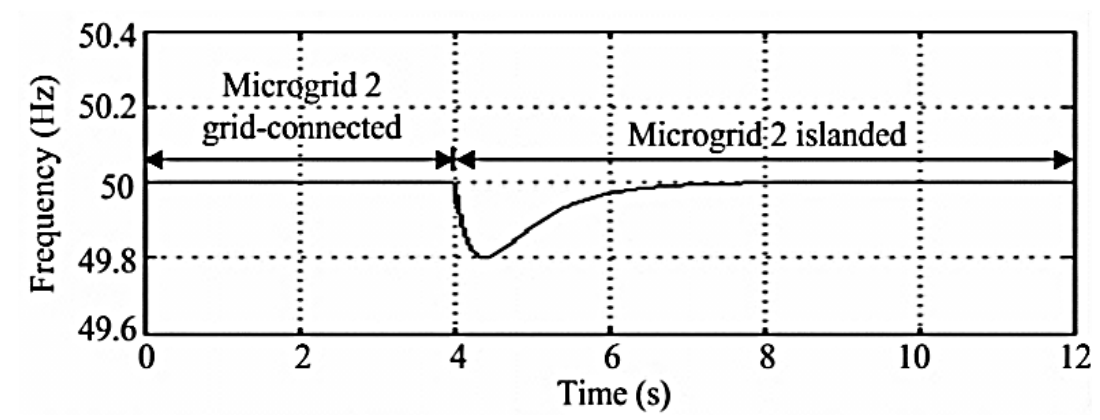

Figure 5-26: System frequency.

\subsection{Conclusion}

In this chapter, a device called SSND has been proposed for an AC/AC multi-area MMG system. A state-space model based MPC algorithm has been developed for the control of DG, series and shunt inverters in the proposed system. The simulation studies have shown that the SSND solves a wide range of power quality issues under several test case scenarios and thus achieves local and non-local harmonic current and reactive power compensation in the AC/AC multi-area MMG system. SSND can also provide real power support during microgrid islanding. The analysis and simulation studies in this paper serve as a fundamental step in the hardware implementation and validation of the system, which will have a scope for further research in the area of power quality improvement in large interconnected microgrid and distribution systems. 


\section{CHAPTER 6 CONCLUSIONS AND RECOMMENDATIONS FOR FUTURE RESEARCH}

\subsection{Conclusions}

To combat climate change and protect the earth from global warming, more and more renewable energy resources need to be used in the future power systems. Microgrid and MMG systems satisfy this need for increased penetration and utilization of these renewable energy sources. Interconnection of microgrids to form MMG systems seems a viable option of establishing large-scale renewable energy systems spread over a wide geographical area. There are many reasons why microgrids are to be interconnected. The main reason of microgrid interconnection is the sharing of power reserves and enhancing the overall system reliability and security. Also, MMG systems can be flexibly operated in grid-connected mode when grid is available, and also in islanded mode when grid is disconnected due to various types of emergency situations.

To bring about the wide-scale deployment of MMG systems as part of future smart grid infrastructure, efficient operational and control strategies need to be developed to manage the power exchange among the interconnected microgrids during different operating scenarios such as islanding, load increase or decrease, load shedding, line outage and inverter or converter failure. In addition, power quality issues and power quality improvement methods need to be addressed in these MMG systems due to the proliferation of non-linear loads and voltage disturbances in the grid voltage.

In Chapter 3 of the thesis, a generalized architecture and control system has been developed for AC/AC multi-area MMG system. This control system consists of a centralized controller for the overall management of the MMG system and local controllers for individual inverters in each microgrid system. An improved MPC algorithm is used by the local controllers to effectively regulate the output voltage and power of these inverters. An underfrequency load shedding scheme is also proposed to restore the system frequency in case of a power shortage in the AC/AC multi-area MMG system. Simulation studies have been conducted to verify the performance of the control system in maintaining the desired power exchanges among the interconnected AC microgrids and regulating the load bus voltage of the islanded AC microgrid. 
Chapter 4 presents a control strategy for an AC/DC multi-area MMG system. In this control strategy, the power exchange among the interconnected AC and DC microgrids is effectively regulated during grid-connected and islanded modes of an interconnected DC microgrid. A state-space model based MPC algorithm has been developed for the local control of multiple converters and inverters in the AC/DC multi-area MMG system. An undervoltage load shedding scheme has also been proposed to restore the load bus voltage of the islanded DC microgrid in case of a power shortage in the AC/DC multi-area MMG system. From the simulation studies conducted, it has been verified that the power exchanges between the interconnected AC and DC microgrids, and the load bus voltage of the islanded DC microgrid can be regulated at their desired reference values using the proposed control strategy.

In Chapter 5 of this thesis, various power quality problems are identified in an AC/AC multiarea MMG system, consisting of linear and non-linear loads. To analyze and solve the power quality problems in an AC/AC multi-area MMG system, new methods different from the conventional methods of power quality improvement are required. A device called SSND has been proposed to compensate the harmonic currents and reactive power of the loads in the interconnected AC microgrids using a new method of non-local harmonic current and reactive power compensation. In addition, this device is also capable of isolating one microgrid from the voltage disturbances occurring in the adjacent microgrid. To incorporate SSND in the AC/AC multi-area MMG system, an analytical study has been conducted first to assess the power flow and power quality control functions of SSND. Then several simulation studies have been conducted to verify the capability of SSND in effective harmonic current and reactive power compensation, and voltage disturbance isolation in the AC/AC multi-area MMG system.

In summary, effective power and voltage control strategies have been developed for different types of the proposed multi-area MMG systems such as AC/AC multi-area MMG system and AC/DC multi-area MMG system. Also, new power quality improvement methods have been developed for the AC/AC multi-area MMG system by incorporating a device called SSND. Finally, different simulation studies have been conducted to validate the performance of these control strategies and power quality improvement schemes in the proposed multi-area MMG systems. 


\subsection{Recommendations for Future Research}

1) The proposed design and control concept of multi-area MMG systems and SSND still needs further validation through experimental studies and hardware implementation. The simulation studies and results obtained in this thesis serve as a preliminary step towards the development of control systems and design architectures for the future experimental verification of the simulation results and real-time hardware implementation of MMG systems and SSND. In the future, hardware implementation of the MMG systems and SSND can be completed using commercially available controllers and industry standard communication/control protocols.

2) The minimization of operating, maintenance and investment costs of the DG and battery units can be considered as part of the future research work to attain an economic operation of the multi-area MMG systems. Various energy management techniques such as load forecasting, battery management and peak shaving can be incorporated into the design of the centralized controller to optimize the overall operation and minimize the operating costs of the MMG systems. Also, energy trading among the interconnected AC and DC microgrid systems is an interesting future research area in MMG systems, which would enable the customers to exploit the benefits of a liberalized electricity market.

3) In the proposed control system for the MMG systems, a high speed and highly reliable communication network is needed for the effective and coordinated operation of the centralized and local controllers. To reduce the communication needs of this control system, other control methods such as decentralized and distributed control methods can be considered for the control and management of the MMG systems in the future research works.

4) As the main focus of the proposed research works is to understand the control philosophy of the MMG systems, the DG units in the proposed research works are assumed to be constant DC sources over the simulation duration. Thus, to understand the impact of variability of power outputs of the DG units such as PV and wind turbines on the performance of the proposed control strategies in the MMG systems, real-time characteristics and dynamic responses of these DG units can be considered in the future hardware implementation of the MMG systems. 
5) In the present research works, the primary attention is given mainly to the islanded operation of the interconnected AC or DC microgrid and the transition of the microgrid from islanded to grid-connected mode is not discussed. Thus, different operating procedures for reconnection, synchronization and black-start can be considered in the future research works on MMG systems.

6) With the advancement of DC transmission in the future, medium voltage DC (MVDC) lines and high voltage DC (HVDC) lines can be used to interconnect microgrid systems separated by hundreds of kilometres apart to achieve economic efficiency over using interconnecting AC transmission lines. New control schemes and strategies can be developed for the reliable and efficient operation of such interconnected microgrid systems, leading to new frontiers of research thus resulting in the overlapping of multiple research areas in MMG systems, and MVDC and HVDC technologies.

7) Protection of multi-area MMG systems is also an important future research topic to ensure the safety of end-users and secure operation of different equipments used in the MMG systems. As the standards for DC microgrid protection are still not fully developed, more research needs to be done to understand and evaluate the different protection schemes for an AC/DC multi-area MMG system.

8) In the future, nuances of power system unbalance and different types of voltage unbalance such as three-phase voltage unbalance and single-phase voltage unbalance will be considered in the proposed AC/AC and AC/DC multi-area MMG systems. 


\section{REFERENCES}

[1] N. Hatziargyriou, Microgrids: Architectures and Control. Wiley, 2014.

[2] R. Lasseter, A. Akhil, C. Marnay, J. Stephens, J. Dagle, R. Guttromson, A. S. Meliopoulous, R. Yinger, and J. Eto (2002), White Paper on Integration of Distributed Energy Resources - The CERTS MicroGrid Concept [Online]. Available: www.pserc.wisc.edu.

[3] S. Chakraborty, M. G. Simoes, and W. E. Kramer, Power Electronics for Renewable and Distributed Energy Systems. Springer, 2013.

[4] A. Keyhani, Design of Smart Power Grid Renewable Energy Systems. Wiley, 2016.

[5] J. A. Pecas Lopes, C. L. Moreira, and A. G. Madureira, "Defining control strategies for microgrids islanded operation,” IEEE Trans. Power Systems, vol. 21, no. 2, pp. 916924, May 2006.

[6] Desertec Foundation (2016) [Online]. Available: http://www.desertec.org.

[7] M. J. Hossain, M. A. Mahmud, F. Milano, S. Bacha, and A. Hably, "Design of robust distributed control for interconnected microgrids," IEEE Trans. Smart Grid, vol. 7, no. 6, pp. $2724-2735$, Nov. 2016.

[8] S. Chowdhury, S. P. Chowdhury, and P. Crossley, Microgrids and Active Distribution Networks. IET, 2009.

[9] C. C. Liu, S. McArthur, and S. J. Lee, Smart Grid Handbook. Wiley, 2016.

[10] A. G. Madureira, "Coordinated and voltage management of distribution networks with multi-microgrids," Ph.D. dissertation, Dept. Elect. and Comp. Eng., Univ. of Porto, Portugal, 2010.

[11] G. N. Korres, N. D. Hatziargyriou, and P. J. Katsikas, "State estimation in multimicrogrids," European Transactions on Electrical Power, vol. 21, no. 2, pp. 1178-1199, Mar. 2011.

[12] A. G. Madureira, J. C. Pereira, N. J. Gil, J. A. P. Lopes, G. N. Korres, and N. D. Hatziargyriou, "Advanced control and management functionalities for multimicrogrids," European Transactions on Electrical Power, vol. 21, no. 2, pp. 1159-1177, Mar. 2011.

[13] United Nations (2006), Multi-dimensional issues in international electric power grid interconnections [Online]. Available: http://sustainabledevelopment.un.org.pdf. 
[14] L. Che, X. Zhang and M. Shahidehpour, "Optimal interconnection planning of community microgrids with renewable energy sources," IEEE Trans. Smart Grid, vol. PP, no. 99, pp. 1-10, Jul. 2015.

[15] D. E. Olivares, C. A. Canizares, and M. Kazerani, “A centralized energy management system for isolated microgrids," IEEE Trans. Power Systems, vol. 5, no. 4, pp. 18641875, Jul. 2014.

[16] D. E. Olivares, C. A. Canizares, and M. Kazerani, "A centralized optimal energy management system for microgrids," in Proc. IEEE Power and Energy Society General Meeting, Jul. 2011, pp. 1-6.

[17] W. Su and J. Wang, "Energy management systems in microgrid operations," Electricity Journal, vol. 25, no. 8, pp. 45-60, Oct. 2012.

[18] K. T. Tan, X. Y. Peng, P. L. So, Y. C. Chu, and M. Z. Q. Chen, "Centralized control for parallel operation of distributed generation inverters in microgrids," IEEE Trans. Smart Grid, vol. 3, no. 4, pp. 1977-1987, Dec. 2012.

[19] M. Prodanovic and T. C. Green, "High-quality power generation through distributed control of a power park microgrid," IEEE Trans. Industrial Electronics, vol. 53, no. 5, pp. 1471-1482, Oct. 2006.

[20] J. Kim, J. M. Guerrero, P. Rodriguez, R. Teodorescu, and K. Nam, "Mode adaptive droop control with virtual output impedances for an inverter-based flexible AC microgrid," IEEE Trans. Power Electronics, vol. 26, no. 3, pp. 689-701, Mar. 2011.

[21] J. Shah, B. F. Wollenberg, and N. Mohan, "Decentralized power flow control for a smart micro-grid," in Proc. IEEE Power and Energy Society General Meeting, Jul. 2011, pp. 1-6.

[22] F. Shahnia, R. Majumder, A. Ghosh, G. Ledwich, and F. Zare, "Operation and control of a hybrid microgrid containing unbalanced and nonlinear loads," Electric Power Systems Research, vol. 80, no. 8, pp. 954-965, Aug. 2010.

[23] H. Liang, B. J. Choi, W. Zhuang, and X. Shen, "Stability enhancement of decentralized inverter control through wireless communications in micro-grids," IEEE Trans. Smart Grid, vol. 4, no. 1, pp. 321-331, Mar. 2013.

[24] Y. Gu, X. Xiang, W. Li, and X. He, "Mode-adaptive decentralized control for renewable DC microgrid with enhanced reliability and flexibility," IEEE Trans. Power Electronics, vol. 29, no. 9, pp. 5072-5080, Sep. 2014. 
[25] N. Cai, X. Xu, and J. Mitra, "A hierarchical multi-agent control scheme for a black start-capable microgrid," in Proc. IEEE Power and Energy Society General Meeting, Jul. 2011, pp. 1-7.

[26] A. L. Dimeas and N. D. Hatziargyriou, "Operation of a multiagent system for microgrid control,” IEEE Trans. Power Systems, vol. 20, no. 3, pp. 1447-1455, Aug. 2005.

[27] C. M. Colson and M. H. Nehrir, "Algorithms for distributed decision-making for multiagent microgrid power management," in Proc. IEEE Power and Energy Society General Meeting, Jul. 2011, pp. 1-8.

[28] T. Logenthiran, D. Srinivasan, A. M. Khambadkone, and H. N. Aung, "Multiagent system for real-time operation of a microgrid in real-time digital simulator," IEEE Trans. Smart Grid, vol. 3, no. 2, pp. 925-933, Jun. 2012.

[29] C. X. Dou and B. Liu, "Multi-agent based hierarchical hybrid control for smart microgrid," IEEE Trans. Smart Grid, vol. 4, no. 2, pp. 771-778, Jun. 2013.

[30] S. Parhizi, H. Lotfi, A. Khodaei, and S. Bahramirad, "State of art in research on microgrids: a review," IEEE Access, vol. 3, pp. 890-925, Jun. 2015.

[31] M. H. Rashid, Power Electronics Handbook. Elsevier, 2011.

[32] M. P. Kazmierkowski, R. Krishnan, and F. Blaabjerg, Control in Power Electronics. Academic Press, 2002.

[33] J. Holtz, "Pulsewidth modulation electronic power conversion," Proceedings of the IEEE, vol. 82, no. 8, pp. 1194-1214, Aug. 1994.

[34] N. Mohan, T. M. Undeland, and W. P. Robbins, Power Electronics. Wiley, 2003.

[35] F. Blaabjerg, R. Teodorescu, M. Liserre, and A. V. Timbus, "Overview of control and grid synchronization for distributed power generation systems," IEEE Trans. Industrial Electronics, vol. 53, no. 5, pp. 1398-1409, Oct. 2006.

[36] J. Rodriguez and P. Cortes, Predictive Control of Power Converters and Electrical Drives. Wiley, 2012.

[37] P. Cortes, M. P. Kazmierkowski, R. M. Kennel, D. E. Quevedo, and J. Rodriguez, "Predictive control in power electronics and drives," IEEE Trans. Industrial Electronics, vol. 55, no. 12, pp. 4312-4324, Dec. 2008.

[38] J. Holtz and S. Stadtfeld, "A predictive controller for the stator current vector of AC machines fed from a switched voltage source," in Proc. International Power Electronics Conference, Tokyo, 1983, pp. 1665-1675.

[39] T. Kawabata, T. Miyashita, and Y. Yamamoto, "Dead beat control of three phase PWM inverter," IEEE Trans. Power Electronics, vol. 5, no. 1, pp. 21-28, Jan. 1990. 
[40] O. Kukrer, "Discrete-time current control of voltage-fed three-phase PWM inverters," IEEE Trans. Industrial Electronics, vol. 11, no. 2, pp. 260-269, Mar. 1996.

[41] U. Ammann, R. Vargas, S. Rees, J. Serra, and J. R. Steilow, “An analytical approach to steady-state current control properties of power converters featuring discrete-time switching," in Proc. Power Electronics Specialists Conference, Jun. 2008, pp. 25352542.

[42] S. Kouro, P. Cortes, R. Vargas, U. Ammann, and J. Rodríguez, "Model predictive control - A simple and powerful method to control power converters," IEEE Trans. Industrial Electronics, vol. 56, no. 6, pp. 1826-1838, Jun. 2009.

[43] P. Cortez, G. Ortiz, J. I. Yuz, J. Rodriguez, S. Vazquez, and L. G. Franquelo, "Model predictive control of an inverter with output LC filter for UPS applications," IEEE Trans. Industrial Electronics, vol. 56, no. 6, pp. 1875-1883, Jun. 2009.

[44] J. Rodriguez, P. Cortez, R. Kennel, and M. P. Kazmierkowski, "Model predictive control - a simple and powerful method to control power converters," in Proc. Power Electronics and Motion Control Conference, pp. 41-49, May 2009.

[45] J. M. Guerrero, J. C. Vasquez, J. Matas, L. G. de Vicuña, and M. Castilla, "Hierarchical control of droop-controlled AC and DC microgrids - a general approach towards standardization,” IEEE Trans. Ind. Electronics, vol. 58, no. 1, pp. 158-172, Jan. 2011.

[46] X. Yu, A. M. Khambadkone, H. Wang, and S. T. S. Terence, "Control of parallelconnected power converters for low-voltage microgrid - part I: A hybrid control architecture," IEEE Trans. Power Electronics, vol. 25, no. 12, pp. 2962-2970, Dec. 2010.

[47] E. Rokrok and M. E. H. Golshan, "Adaptive voltage droop scheme for voltage source converters in an islanded multibus microgrid," IET Generation, Transmission and Distribution, vol. 4, no. 5, pp. 562-578, May 2010.

[48] M. B. Delghavi and A. Yazdani, "An adaptive feedforward compensation for stability enhancement in droop-controlled inverter-based microgrids," IEEE Trans. Power Delivery, vol. 26, no. 3, pp. 1764-1773, Jul. 2011.

[49] K. S. Low and R. Cao, "Model predictive control of parallel connected inverters for uninterruptible power supplies," in Proc. Power Electronics Specialists Conference, Jun. 2006, pp. 1-6.

[50] K. S. Low and R. Cao, "Model predictive control of parallel connected inverters for uninterruptible power supplies," IEEE Trans. Industrial Electronics, vol. 55, no. 8, pp. 2884-2893, Aug. 2008. 
[51] L. Fusheng, L. Ruisheng, and Z. Fengquan, Microgrid Technology and Engineering Application. Elsevier, 2016.

[52] H. Karimi, H. Nikkhajoei, and M. R. Iravani, "Control of an electronically-coupled distributed resource unit subsequent to an islanding event," IEEE Trans. Power Delivery, vol. 23, no. 1, pp. 493-501, Jan. 2008.

[53] F. Katiraei, M. R. Iravani, and P. W. Lehn, "Micro-grid autonomous operation during and subsequent to islanding process," IEEE Trans. Power Delivery, vol. 20, no. 1, pp. 248-257, Jan. 2005.

[54] Y. Li, D. M. Vilathgamuwa, and P. Loh, "Design, analysis, and real-time testing of a controller for multibus microgrid system," IEEE Trans. Power Electronics, vol. 19, no. 5, pp. 1195-1204, Sep. 2004.

[55] P. Basak, A. K. Saha, S. Chowdhury, and S. P. Chowdhury, "Microgrid: Control techniques and modeling," in Proc. IEEE $44^{\text {th }}$ International Universities Power Engineering Conference, 2009, pp. 1-5.

[56] Y. Xu, H. Li, and L. M. Tolbert, "Inverter-based microgrid control and stable islanding transition," in Proc. IEEE Energy Conversion Congress and Exposition, 2012, pp. 2374-2380.

[57] F. Gao and M. R. Iravani, “A control strategy for a distributed generation unit in gridconnected and autonomous modes of operation," IEEE Trans. Power Delivery, vol. 23, no. 2, pp. 850-859, Apr. 2008.

[58] C. Hou, X. Hu, and D. Hui, "Hierarchical control techniques applied in micro-grid," in Proc. International Conference on Power System Technology, 2010, pp. 1-5.

[59] I. J. Balaguer, Q. Lei, S. Yang, U. Supatti, and F. Z. Peng, "Control for grid-connected and intentional islanding operations of distributed power generation," IEEE Trans. Industrial Electronics, vol. 58, no. 1, pp. 147-157, Jan. 2011.

[60] F. Katiraei, C. Abbey, S. Tang, and M. Gauthier, "Planned islanding on rural feeders utility perspective," in Proc. IEEE Power and Energy Society General Meeting, Jul. 2008, pp. 1-6.

[61] A. H. K. Alaboudy, H. H. Zeineldin, and J. Kirtley, "Microgrid stability characterization subsequent to fault-triggered islanding incidents," IEEE Trans. Power Delivery, vol. 27, no. 2, pp. 658-669, Apr. 2012.

[62] J. Hossain and A. Mahmud, Renewable Energy Integration: Challenges and Solutions. Springer-Verlag, 2014. 
[63] L. Che, M. Khodayar, and M. Shahidehpour, "Only connect: microgrids for distribution system restoration," IEEE Power Energy Magazine, vol. 12, no. 1, pp. 70-81, Jan./Feb. 2014

[64] J. M. Guerrero, L. Hang, and J. Uceda, "Control of distributed uninterruptible power supply systems," IEEE Trans. Industrial Electronics, vol. 55, no. 8, pp. 2845-2859, Aug. 2008.

[65] Z. Bo, Z. Xuesong, and C. Jian, "Integrated microgrid laboratory system," IEEE Trans. Power Systems, vol. 27, no. 4, pp. 2175-2185, Nov. 2012.

[66] P. Piagi and R. H. Lasseter, "Autonomous control of microgrids," in Proc. IEEE Power Engineering Society General Meeting, Jun. 2006.

[67] G. Turner, J. P. Kelley, C. L. Storm, D. A. Wetz, and W. Lee, "Design and active control of a microgrid test bed," IEEE Trans. Smart Grid, vol. 6, no. 1, pp. 73-81, Jan. 2015.

[68] I. U. Nutkani, P. Loh, and F. Blaabjerg, "Distributed operation of interlinked AC microgrids with dynamic active and reactive power tuning," IEEE Trans. Industry Applications, vol. 49, no. 5, pp. 2188-2196, Sep./Oct. 2013.

[69] S. Moayedi and A. Davoudi, "Distributed tertiary control of DC microgrid clusters," IEEE Trans. Power Electronics, vol. 31, no. 2, pp. 1717-1733, Feb. 2016.

[70] X. Liu, P. Wang, and P. C. Loh, "A hybrid AC/DC microgrid and its coordination control," IEEE Trans. Smart Grid, vol. 2, no. 2, pp. 278-286, Mar. 2011.

[71] L. Che, M. Shahidehpour, A. Alabulwahab, and Y. A. Turki, "Hierarchical coordination of a community microgid with AC and DC microgrids," IEEE Trans. Smart Grid, vol. 6, no. 6, pp. 3042-3051, Nov. 2015.

[72] H. Zhang, J. Zhou, Q. Sun, J. M. Guerrero, and D. Ma "Data driven control for interlinked AC/DC microgrids via model-free adaptive control and dual droop control," IEEE Trans. Smart Grid, vol. PP, no. 99, pp. 1-15, Dec. 2015.

[73] N. Eghtedarpour and E. Farjah, "Power control and management in a hybrid AC/DC microgrid," IEEE Trans. Smart Grid, vol. 5, no. 3, pp. 1495-1505, May 2014.

[74] S. Borlase, Smart Grids Infrastructure Technology and Solutions. Taylor and Francis, 2013.

[75] C. W. Gellings, “A globe-spanning supergrid,” IEEE Spectrum Magazine, pp. 44-51, Aug. 2015.

[76] P. S. Revuelta, S. P. Litran, and J. P. Thomas, Active Power Line Conditioners Design, Simulation and Implementation for Improving Power Quality. Elsevier, 2016. 
[77] R. C. Dugan, M. F. McGranagan, S. Santoso, and H. W. Beaty, Electrical Power Systems Quality. Mcgraw-Hill, 2012.

[78] K. T. Tan, P. L. So, Y. C. Chu, and M. Z. Q. Chen, “A flexible AC distribution system device for a microgrid," IEEE Trans. Energy Conversion, vol. 28, no. 3, pp. 601-610, Sep. 2013.

[79] S. Chakraborty and M. G. Simoes, "Experimental evaluation of active filtering in a single-phase high-frequency AC microgrid," IEEE Trans. Energy Conversion, vol. 24, no. 3, pp. 673-682, Sep. 2009.

[80] A. Ghosh and G. Ledwich, Power Quality Enhancement Using Custom Power Devices. Springer, 2002.

[81] European Standard UNE-EN 50160, Voltage Characteristics of Electricity Supplied by Public Distribution Systems, 2010.

[82] IEEE Recommended Practice and Requirements for Harmonic Control in Electric Power Systems, IEEE Standard 519-2014, Mar. 2014.

[83] J. Nastran, R. Cajhen, M. Seliger, and P. Jereb, "Active power filter for nonlinear AC loads,” IEEE Trans. Power Electronics, vol. 9, no. 1, pp. 92-96, Jan. 1994.

[84] V. B. Bhavaraju and P. N. Enjeti, "Analysis, design of an active power filter for balancing unbalanced loads," IEEE Trans. Power Electronics, vol. 8, no. 4, pp. 640647, Oct. 1993.

[85] A. Cavallini and G. C. Montanari, "Compensation strategies for shunt active-filter control,” IEEE Trans. Power Electronics, vol. 9, no. 6, pp. 587-593, Nov. 1994.

[86] A. Campos, G. Joos, P. D. Ziogas, and J. F. Lindsay, "Analysis, design of a series voltage unbalance compensator based on a three-phase VSI operating with unbalanced switching functions," IEEE Trans. Power Electronics, vol. 9, no. 3, pp. 269-274, May 1994.

[87] N. H. Woodley, L. Morgan, and A. Sundaram, "Experience with an inverter-based dynamic voltage restorer," IEEE Trans. Power Delivery, vol. 14, no. 3, pp. 1181-1186, Jul. 1999.

[88] J. G. Nielsen, M. Newman, and F. Blaabjerg, "Control and testing of a dynamic voltage restorer (DVR) at medium voltage level," IEEE Trans. Power Delivery, vol. 19, no. 3, pp. 806-813, May. 2004.

[89] H. Fujita and H. Akagi, "A practical approach to harmonic compensation in power systems-series connection of passive and active filters," IEEE Trans. Industry Applications, vol. 27, no. 6, pp. 1020-1025, Nov./Dec. 1991. 
[90] H. Akagi, E. H. Watanabe, and M. Aredes, Instantaneous Power Theory and Applications to Power Conditioning. Wiley-IEEE Press, 2007.

[91] M. Rastogi, N. Mohan, and A. A. Edris, "Hybrid-active filtering of harmonic currents in power systems," IEEE Trans. Power Delivery, vol. 10, no. 4, pp. 1994-2000, Oct. 1995.

[92] S. A. Moran and M. B. Brennen, "Active power line conditioner with fundamental negative sequence compensation," U.S. Patent 5384 696, Jan. 1995.

[93] B. Singh, A. Chandra, and K. A. Haddad, Power Quality: Problems and Mitigation Techniques. Wiley, 2014.

[94] V. Khadkikar and A. Chandra, "UPQC-S: A novel concept of simultaneous voltage sag/swell and load reactive power compensations utilizing series inverter of UPQC," IEEE Trans. Power Electronics, vol. 26, no. 9, pp. 2414-2425, Sep. 2011.

[95] H. R. Mohammadi, R. Y. Varjani, and H. Mokhtari, "Multiconverter unified powerquality conditioning system: MC-UPQC," IEEE Trans. Power Delivery, vol. 24, no. 3, pp. 1679-1686, Jul. 2009.

[96] K. H. Kwan, Y. C. Chu, and P. L. So, "Model-based H- $\infty$ control of a unified power quality conditioner," IEEE Trans. Industrial Electronics, vol. 56, no. 7, pp. 2493-2504, Jul. 2009.

[97] S. Chakraborty and M. G. Simoes, "Experimental evaluation of active filtering in a single-phase high-frequency AC microgrid," IEEE Trans. Energy Conversion, vol. 24, no. 3, pp. 673-682, Sep. 2009.

[98] V. Khadkikar and A. Chandra, "A novel structure for three-phase four-wire distribution system utilizing unified power quality conditioner (UPQC)," IEEE Trans. Industry Applications, vol. 45, no. 5, pp. 1897-1902, Sep./Oct. 2009.

[99] G. Casaravilla, A. Salvia, C. Briozzo, and E. H. Watanabe, "Selective filter with optimum remote harmonic distortion control," IEEE Trans. Power Delivery, vol. 19, no. 4, pp. 1990-1997, Oct. 2004.

[100] S. X. Chen, Y. S. F. Eddy, H. B. Gooi, M. Q. Wang, and S. F. Lu, "A centralized reactive power compensation system for LV distribution networks," IEEE Trans. Power Systems, vol. 30, no. 1, pp. 274-284, Jan. 2015.

[101] A. Moreno-Munoz, Power Quality Mitigation Technologies in a Distributed Environment. Springer, 2007.

[102] A. Baggini, Handbook of Power Quality. Wiley, 2008.

[103] P. Kundur, Power System Stability and Control. McGraw-Hill, 1994. 
[104] D. E. Olivares, A. Mehrizi-Sani, A. H. Etemadi, et al., "Trends in microgrid control," IEEE Trans. Smart Grid, vol. 5, no. 4, pp. 1905-1919, Jul. 2014.

[105] Transmission Code Energy Market Authority of Singapore [Online]. Available: http://www.ema.gov.sg/cmsmedia/Licensees.pdf.

[106] M. J. Sanjari, A. H. Yatim, and G. B. Gharehpetian, "Online dynamic security assessment of microgrids before intentional islanding occurrence", Neural Computing and Applications, vol. 26, pp. 659-668, Apr. 2015.

[107] Underfrequency Issues Work Group WSCC Technical Studies Subcommittee WSCC Coordinated Off-nominal Frequency Load Shedding and Restoration Plan [Online] Available: https://www.wecc.biz/relibility/wecc\%20ONF\%20Report\%20July.pdf.

[108] P. M. Anderson and M. Mirheydar, “An adaptive method for setting underfrequency load shedding relays," IEEE Trans. Power Systems, vol. 7, no. 2, pp. 647-655, May 1992.

[109] K. T. Tan, B. Sivaneasan, X. Y. Peng, and P. L. So, "Control and operation of a DC grid-based wind power generation system in a microgrid," IEEE Trans. Energy Conversion, vol. 31, no. 2, pp. 496-505, Jun. 2016.

[110]L. Wang, Model Predictive Control System Design and Implementation Using MATLAB. Springer, 2009.

[111] J. Xiao, L. Seryawan, P. Wang, and C. Jin, "Power capacity-based bus-voltage region partition and online droop coefficient tuning for real-time operation of DC microgrids," IEEE Trans. Energy Conversion, vol. 30, no. 4, pp. 1338-1347, Dec. 2015.

[112] Under Voltage Load Sheding Guidelines [Online]. Available: https://www.wecc.biz/Reliability/Undervoltage\%20Load\%20Shedding\%20Guidelines .pdf.

[113] D. Chen, L. Xu, and L. Yao, "DC voltage variation based autonomous control of DC microgrids," IEEE Trans. Power Delivery, vol. 28, no. 2, pp. 637-648, Apr. 2013.

[114] Residential \& Commercial Use of DC Power [Online]. Available: http://portal.emergealliance.org/DesktopModules/Inventure_Document/FileDownload.a spx?ContentID=20144.

[115] L. H. Tey, P. L. So, and Y. C. Chu, "Improvement of power quality using adaptive shunt active filter," IEEE Trans. Power Delivery, vol. 20, no. 2, pp. 1558-1568, Apr. 2005 .

[116] K. R. Rao, D. N. Kim, and J. J. Hwang, Fast Fourier Transform: Algorithms and Applications. Springer, 2010. 
[117] A. Yazdani and R. Iravani, Voltage Source Converters in Power Systems. Wiley, 2010.

[118] K. T. Tan, P. L. So, Y. C. Chu, and M. Z. Q. Chen, "Coordinated control and energy management of distributed generation inverters in a microgrid," IEEE Trans. Power Delivery, vol. 28, no. 2, pp. 704-713, Apr. 2013.

[119] N, Pogaku, M. Prodanovic, and T. C. Green, "Modelling, analysis and testing of autonomous operation of an inverter-based microgrid," IEEE Trans. Power Electronics, vol. 22, no. 2, pp. 613-625, Mar. 2007.

[120] M. J. Sanjari and G. B. Gharehpetian, "Unified framework for frequency and voltage control of autonomous microgrids," IET Generation, Transmission and Distribution, vol. 7, no. 9, pp. 965-972, Sep. 2013. 


\section{APPENDIX A}

\section{Derivation of State-Space Power System Model of the AC/AC Multi-Area MMG System}

The single-phase equivalent circuit of the proposed AC/AC multi-Area MMG system, which is conceptually based on [117], [118], is shown in Figure A-1. For the purpose of simplicity, only two interconnected microgrids are considered in the proposed MMG system but the derivation of the state-space power system model given here can be extended for a generalized case of ' $n$ ' number of microgrids shown in Figure 3-1 in Chapter 3.

In Figure A-1, inverters 1 and 2 represent DG and battery inverters respectively in microgrid 1. Inverters 3 and 4 represent DG and battery inverters respectively in microgrid 2. $R_{1}, R_{2}, R_{3}$ and $R_{4}$ represent the internal loss resistances of inverters $1,2,3$ and 4 respectively. $L_{f 1}, L_{f 2}, L_{f 3}$ and $L_{f 4}$ are the filter inductances of inverters 1, 2, 3 and 4 respectively. $C_{f 1}, C_{f 2}, C_{f 3}$ and $C_{f 4}$ are the filter capacitances of inverters $1,2,3$ and 4 respectively. $i_{1}, i_{2}, i_{3}$ and $i_{4}$ are the filter inductance currents in inverters $1,2,3$ and 4 respectively. $V_{d c 1}, V_{d c 2}, V_{d c 3}$ and $V_{d c 4}$ are the input DC voltages of inverters 1, 2, 3 and 4 respectively. $V_{L 1}$ and $V_{L 2}$ are the load 1 and load 2 voltages respectively. $u_{1}, u_{2}, u_{3}$ and $u_{4}$ are the switching signals given to inverters $1,2,3$ and 4 respectively. $i_{D G 1}, i_{D G 2}, i_{D G 3}$ and $i_{D G 4}$ are the output currents of inverters $1,2,3$ and 4 respectively. $i_{G 1}$ and $i_{G 2}$ are the distribution grid 1 and distribution grid 2 currents respectively. $R_{t 1}$ and $L_{t 1}$ represent the line 1 resistance and inductance respectively. $R_{L 1}$ and $R_{L 2}$ represent the load 1 and load 2 resistances respectively. $L_{L 1}$ and $L_{L 2}$ represent the load 1 and load 2 inductances respectively. $i_{G 1}$ and $i_{G 2}$ are the distribution grid 1 and distribution grid 2 currents respectively.

The state-space power system model of the MMG system is derived by compiling the differential equations of inverters, line and loads. The principle of compilation of the differential equations of inverters, line and loads is based on [119], [120]. The derivation of state-space power system model of the MMG system from the differential equations is given as follows: 


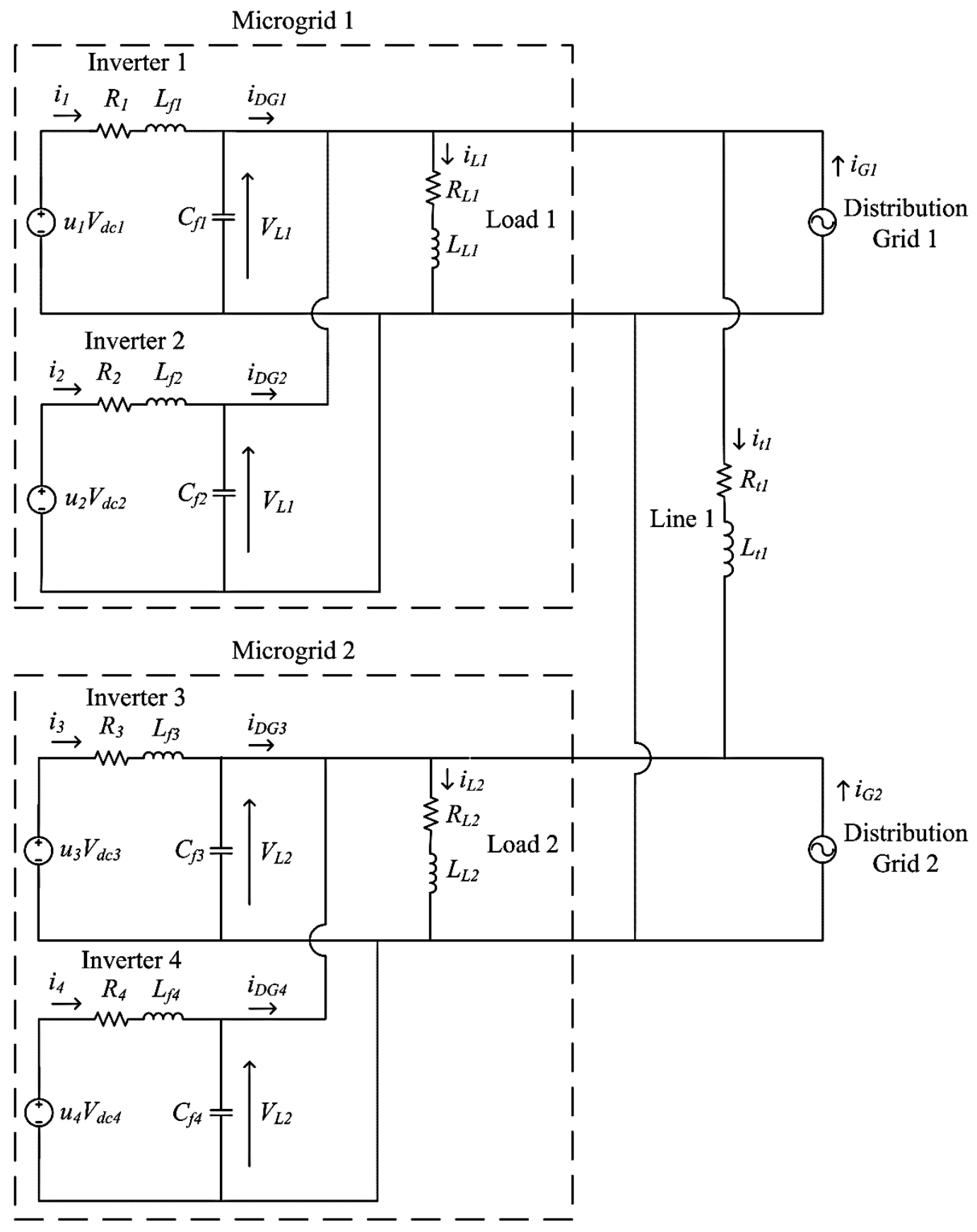

Figure A-1: Single-phase equivalent circuit of the MMG system.

1) Differential equations of inverters

By applying Kirchhoff's voltage law to the single-phase equivalent circuit, the following equations are obtained:

$$
u_{1} V_{d c 1}=R_{1} i_{1}+L_{f 1} \frac{d i_{1}}{d t}+V_{L 1}
$$




$$
\begin{aligned}
& u_{2} V_{d c 2}=R_{2} i_{2}+L_{f 2} \frac{d i_{2}}{d t}+V_{L 1} \\
& u_{3} V_{d c 3}=R_{3} i_{3}+L_{f 3} \frac{d i_{3}}{d t}+V_{L 2} \\
& u_{4} V_{d c 4}=R_{4} i_{4}+L_{f 4} \frac{d i_{4}}{d t}+V_{L 2}
\end{aligned}
$$

From (A.1) to (A.4), the following equations can be derived.

$$
\begin{aligned}
& \dot{i}_{1}^{\prime}=\frac{d i_{1}}{d t}=\frac{u_{1} V_{d c 1}}{L_{f 1}}-\frac{R_{1} i_{1}}{L_{f 1}}-\frac{V_{L 1}}{L_{f 1}} \\
& i_{2}^{\prime}=\frac{d i_{2}}{d t}=\frac{u_{2} V_{d c 2}}{L_{f 2}}-\frac{R_{2} i_{2}}{L_{f 2}}-\frac{V_{L 1}}{L_{f 2}} \\
& \dot{i}_{3}^{\prime}=\frac{d i_{3}}{d t}=\frac{u_{3} V_{d c 3}}{L_{f 3}}-\frac{R_{3} i_{3}}{L_{f 3}}-\frac{V_{L 2}}{L_{f 3}} \\
& \dot{i}_{4}^{\prime}=\frac{d i_{4}}{d t}=\frac{u_{4} V_{d c 4}}{L_{f 4}}-\frac{R_{4} i_{4}}{L_{f 4}}-\frac{V_{L 2}}{L_{f 4}}
\end{aligned}
$$

Similarly, by applying Kirchhoff's current law to the single-phase equivalent circuit, the following equations are obtained:

$$
\begin{aligned}
& C_{f 1} \frac{d V_{L 1}}{d t}=i_{1}-i_{D G 1} \\
& C_{f 2} \frac{d V_{L 1}}{d t}=i_{2}-i_{D G 2} \\
& C_{f 3} \frac{d V_{L 2}}{d t}=i_{3}-i_{D G 3} \\
& C_{f 4} \frac{d V_{L 2}}{d t}=i_{4}-i_{D G 4}
\end{aligned}
$$

The currents $i_{D G 1}$ and $i_{D G 3}$ can be expressed as follows:

$$
\begin{aligned}
& i_{D G 1}=i_{L 1}-i_{D G 2}-i_{G 1}+i_{t 1} \\
& i_{D G 3}=i_{L 2}-i_{D G 4}-i_{G 2}-i_{t 1}
\end{aligned}
$$


By substituting these currents from (A.13) and (A.14) into (A.9) and (A.11), the following equations are obtained:

$$
\begin{aligned}
& V_{L 1}^{\prime}=\frac{d V_{L 1}}{d t}=\frac{i_{1}}{C_{f 1}}-\frac{i_{L 1}}{C_{f 1}}+\frac{i_{D G 2}}{C_{f 1}}+\frac{i_{G 1}}{C_{f 1}}-\frac{i_{t 1}}{C_{f 1}} \\
& V_{L 2}^{\prime}=\frac{d V_{L 2}}{d t}=\frac{i_{3}}{C_{f 3}}-\frac{i_{L 2}}{C_{f 3}}+\frac{i_{D G 4}}{C_{f 3}}+\frac{i_{G 2}}{C_{f 3}}+\frac{i_{t 1}}{C_{f 3}}
\end{aligned}
$$

Thus, equations (A.5) to (A.8) represent the differential current equations of the inverters and equations (A.15) to (A.16) represent the differential voltage equations of the inverters.

2) Differential equation of line 1

By applying Kirchhoff's voltage law to the single-phase equivalent circuit, the following differential equations are obtained:

$$
\begin{aligned}
V_{L 1} & =R_{t 1} i_{t 1}+L_{t 1} \frac{d i_{t 1}}{d t}+V_{L 2} \\
i_{t 1}^{\prime} & =\frac{d i_{t 1}}{d t}=\frac{V_{L 1}}{L_{t 1}}-\frac{R_{t 1} i_{t 1}}{L_{t 1}}-\frac{V_{L 2}}{L_{t 1}}
\end{aligned}
$$

3) Differential equations of loads 1 and 2

Due to high sampling frequency used in the proposed MPC method for the inverters in the MMG system, the load currents can be assumed as follows:

$$
i_{L 1}^{\prime}=\frac{d i_{L 1}}{d t}=0 ; i_{L 2}^{\prime}=\frac{d i_{L 2}}{d t}=0
$$

Therefore by compiling the differential equations of inverters, line and loads in the MMG system, the state-space power system model of the MMG system is obtained as follows:

$$
\begin{gathered}
\dot{x}=A x+B u+D w \\
y=C x
\end{gathered}
$$


where

$\dot{x}=\left[\begin{array}{lllllllll}i_{1} & i_{2} & i_{3}^{\prime} & i_{4}^{\prime} & V_{L 1}^{\prime} & V_{L 2}^{\prime} & i_{t 1}^{\prime} & i_{L 1}^{\prime} & i_{L 2}^{\prime}\end{array}\right]^{T} ;$

$x=\left[\begin{array}{lllllllll}i_{1} & i_{2} & i_{3} & i_{4} & V_{L 1} & V_{L 2} & i_{t 1} & i_{L 1} & i_{L 2}\end{array}\right]^{T}$;

$u=\left[\begin{array}{llll}u_{1} & u_{2} & u_{3} & u_{4}\end{array}\right]^{T} ; w=\left[\begin{array}{llll}i_{G 1} & i_{G 2} & i_{D G 2} & i_{D G 4}\end{array}\right]^{T} ; \mathrm{y}=\left[\begin{array}{ll}V_{L 1} & V_{L 2}\end{array}\right]^{T}$

$$
A=\left[\begin{array}{ccccccccc}
\frac{-R_{1}}{L_{f 1}} & 0 & 0 & 0 & \frac{-1}{L_{f 1}} & 0 & 0 & 0 & 0 \\
0 & \frac{-R_{2}}{L_{f 2}} & 0 & 0 & \frac{-1}{L_{f 2}} & 0 & 0 & 0 & 0 \\
0 & 0 & \frac{-R_{3}}{L_{f 3}} & 0 & 0 & \frac{-1}{L_{f 3}} & 0 & 0 & 0 \\
0 & 0 & 0 & \frac{-R_{4}}{L_{f 4}} & 0 & \frac{-1}{L_{f 4}} & 0 & 0 & 0 \\
\frac{1}{C_{f 1}} & 0 & 0 & 0 & 0 & 0 & \frac{-1}{C_{f 1}} & \frac{-1}{C_{f 1}} & 0 \\
0 & 0 & \frac{1}{C_{f 3}} & 0 & 0 & 0 & \frac{1}{C_{f 3}} & 0 & \frac{-1}{C_{f 3}} \\
0 & 0 & 0 & 0 & \frac{1}{L_{t 1}} & \frac{-1}{L_{t 1}} & \frac{-R_{t 1}}{L_{t 1}} & 0 & 0 \\
0 & 0 & 0 & 0 & 0 & 0 & 0 & 0 & 0 \\
0 & 0 & 0 & 0 & 0 & 0 & 0 & 0 & 0
\end{array}\right] ;
$$

$$
B=\left[\begin{array}{cccc}
\frac{V_{d c 1}}{L_{f 1}} & 0 & 0 & 0 \\
0 & \frac{V_{d c 2}}{L_{f 2}} & 0 & 0 \\
0 & 0 & \frac{V_{d c 3}}{L_{f 3}} & 0 \\
0 & 0 & 0 & \frac{V_{d c 4}}{L_{f 4}} \\
0 & 0 & 0 & 0 \\
0 & 0 & 0 & 0 \\
0 & 0 & 0 & 0 \\
0 & 0 & 0 & 0 \\
0 & 0 & 0 & 0
\end{array}\right] ;
$$




$$
C=\left[\begin{array}{ccccccccc}
0 & 0 & 0 & 0 & 1 & 0 & 0 & 0 & 0 \\
0 & 0 & 0 & 0 & 0 & 1 & 0 & 0 & 0
\end{array}\right] ; D=\left[\begin{array}{cccc}
0 & 0 & 0 & 0 \\
0 & 0 & 0 & 0 \\
0 & 0 & 0 & 0 \\
0 & 0 & 0 & 0 \\
\frac{1}{C_{f 1}} & 0 & \frac{1}{C_{f 1}} & 0 \\
0 & \frac{1}{C_{f 3}} & 0 & \frac{1}{C_{f 3}} \\
0 & 0 & 0 & 0 \\
0 & 0 & 0 & 0 \\
0 & 0 & 0 & 0
\end{array}\right]
$$

In this state-space power system model, $x$ is the state vector, $\dot{x}$ is the first derivative of $x$ with respect to time, $y$ is the output vector, $u$ is the switching state input vector and $w$ is the disturbance vector. $A, B, C$ and $D$ are the state, input, output and disturbance matrices respectively. 


\section{LIST OF PUBLICATIONS}

\section{Journal Publications}

1. T. John and P. L. So, "Voltage and frequency control during microgrid islanding in a multi-area multi-microgrid system," IET Generation, Transmission and Distribution, vol. 11, no. 6, pp. 1502-1512, May 2017.

2. T. John, Y. Wang, and P. L. So, "A series-shunt network device for an interconnected microgrid system," under first revision in IEEE Trans. Sustainable Energy.

3. T. John and P. L. So, "Voltage and power control in an AC/DC multi-area multimicrogrid system," submitted to IEEE Trans. Energy Conversion. (under review)

4. Y. Wang, T. John, and P. L. So, "Hierarchical control and autonomous operation of islanded microgrids with distributed energy resources," submitted to IEEE Trans. Energy Conversion. (under review)

\section{Conference Publications}

1. T. John, Y. Wang, K. T. Tan, and P. L. So, "Model predictive control of distributed generation inverter in a microgrid," in Proc. IEEE Asia Innovative Smart Grid Technology Conference, Kuala Lumpur, May 2014, pp. 657-662.

2. T. John, Y. Wang, P. L. So, and K. T. Tan, "Coordinated operation of a microgrid with a distribution network device," in Proc. IEEE Asia Innovative Smart Grid Technology Conference, Bangkok, Nov. 2015, pp. 1-6. 